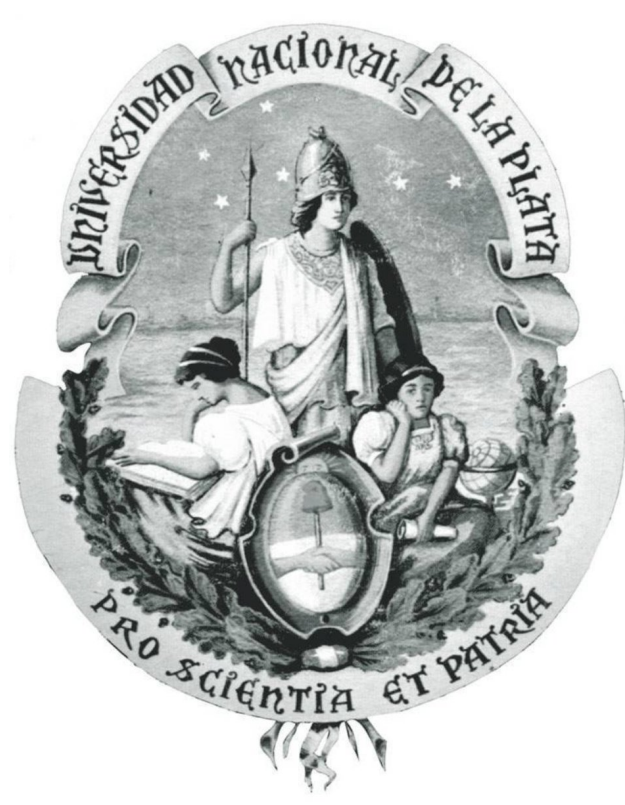

Universidad Nacional de La Plata

Facultad de Ciencias Astronómicas y Geofísicas

Tesis para obtener el grado académico de

Doctor en Astronomía

\title{
Propiedades de los Vientos DE LAS ESTRELLAS SUPERGIGANTES B Y ESTADO EVOLUTIVO
}

Maximiliano Haucke

Directora: Lydia Sonia Cidale.

Co-director: Jorge Panei

La Plata, Argentina

- FeBrero de 2018 - 

Para mis amores, Ayelén y Emilia. 



\section{Agradecimientos}

Transitar este largo camino y concluirlo con esta tesis no habría sido posible sin la ayuda de mucha gente a la cual quiero agradecer que hayan compartido este proceso conmigo...

A Lydia, por ser como mi tercera madre. Por haberme guiado siempre con tranquilidad y cariño. Por brindarme siempre nuevas ideas y posibilidades para hacer lo que me gusta. Por sus consejos multidisciplinarios, y por su ayuda y paciencia en momentos difíciles.

A Roberto, mi gran compañero de oficina, por estar siempre con una sonrisa y largar lo que sea que esté haciendo cada vez que lo necesito. Por aguantarme en mis malos días.

A mi hermana, por diseñarme los pósters más copados de todos con tanto amor y dedicación, sin importar la hora o el día. Y también por crear algunas de las imágenes que aparecen en esta tesis.

A Betina, por su magia a la hora de solucionar todo tipo de problemas burocráticos.

A Yani, Andrea y María por ser unas compañeras de lujo.

A mis colegas y amigos de Valparaíso, Ignacio, Cata, Alex y Michelitus, por tratarme como si estuviera en casa cada vez que tuve que cruzar la cordillera.

A Michaela, por haber realizado en conjunto muchos trabajos que plasman una gran parte de esta tesis. Por compartir su experiencia y sencillez.

A todo el personal del CASLEO, por su amabilidad, trabajo, responsabilidad y profesionalismo desde el primer instante. Por hacer aún más especial el lugar.

A Ayelén, por su amor, por ser una gran compañera y por hacer que todo sea más fácil. Por enseñarme a nunca bajar los brazos. 



\section{Resumen}

La evolución de las estrellas masivas depende fundamentalmente de su masa inicial, pero factores secundarios como la metalicidad, la eyección de materia o la interacción gravitatoria en sistemas binarios, pueden modificar radicalmente las fases evolutivas y las características del entorno estelar. Los mecanismos que favorecen la eyección de materia, tales como la rotación, las pulsaciones, la presión de radiación, y, en especial, las explosiones de supernovas, tienen importantes consecuencias sobre el medio circunestelar e interestelar, aportando grandes cantidades de energía y momento. A su vez, las abundancias químicas superficiales de una estrella masiva se ven modificadas por el enriquecimiento de elementos pesados generados en su interior, que con ayuda del mezclado convectivo externo (MCE, o "dredge up") suben a las capas superiores favoreciendo el desarrollo de inestabilidades, las que influyen en las propiedades de los vientos estelares (Panei 2004.

Un elemento importante en la descripción de la teoría de evolución estelar es la tasa de la pérdida de masa. La pérdida de materia a lo largo de la vida de una estrella puede ser muy grande y favorecer la formación de discos y envolturas. Además, la determinación de las abundancias químicas constituye otro elemento de diagnośtico y verificación de la teoría de evolución estelar. Tal es así que la extensión de la región que posee helio enriquecido puede discriminarse contemplando los procesos de mezcla en la fase de secuencia principal (por efectos de la rotación) y en la fase de supergigante roja por MCE, mientras que los cocientes de abundancias de $\mathrm{C} / \mathrm{N}$ y $\mathrm{O} / \mathrm{N}$ dan información de la extensión de las regiones de elementos procesados por ciclos $\mathrm{CN}$ y ON del ciclo CNO. Entonces, el enriquecimiento por CNO procesado será más importante en las estrellas más masivas y más luminosas Lennon et al. 1993a. Es también de esperar que las supergigantes en la fase de post-supergigante roja tengan más intensificado el CNO procesado. Sin embargo, las anomalías de carbono y nitrógeno en las superficies de las supergigantes B están bien documentadas y dieron lugar al sistema de clasificación espectral BN y BC propuesto por Walborn 1976). Los estudios más recientes mostraron que la materia nuclear procesada, determinada por el cociente $\mathrm{C} / \mathrm{N}$, requiere de un mecanismo más eficiente de mezcla que el predicho por los modelos actuales de estrellas con rotación y pérdida de masa (Firnstein \& Przybilla 2006). La correcta estimación de la composición química es importante al momento de analizar la propia evolución estelar y la dinámica del viento.

El estudio de los vientos de las estrellas supergigantes está basado en la teoría de vientos impulsados por radiación, desarrollada por Castor et al. 1975, y en la cual se adopta una ley de velocidades $\beta$ como aproximación a la solución hidrodinámica del fluido. El 
parámetro $\beta$ define el gradiente de la curva de velocidad del viento y la velocidad terminal de las las partículas, que es la velocidad que éstas alcanzan a distancias muy grandes comparadas con las dimensiones del objeto. Estos dos parámetros, en conjunto con la tasa de pérdida de masa, constituyen los tres parámetros que caracterizan a un viento estelar.

Esta tesis está centrada en el estudio de los vientos de las estrellas supergigantes B y su variabilidad. La importancia de entender el comportamiento de estos objetos es fundamental para comprender la evolución de las estrellas masivas en general y la evolución dinámica y química de las galaxias donde ellas se encuentran. En particular, estos objetos se ubican en las inmediaciones del salto de biestabilidad de temperatura Lamers et al. 1995, región que divide claramente las características de los vientos estelares en dos grupos. Aquí merece destacarse que la teoría estándar sólo explica consistentemente los vientos con $\beta \leq 1$ aunque las observaciones sugieren valores de $\beta$ entre 1 y 3 .

Para estudiar las estrellas supergigantes $B$, se realizó un análisis mediante la comparación de espectros sintéticos con los observados, principalmente el de la línea $\mathrm{H}_{\alpha}$ que, en el rango óptico, es la línea más sensible para derivar los parámetros del viento. Para modelar los perfiles observados se utilizó el código de transporte radiativo FASTWIND, desarrollado por Puls et al. 2005.

En esta tesis estudiamos, por un lado, una muestra de 19 estrellas supergigantes B, cubriendo todos los subtipos espectrales, desde las más calientes (B0) hasta las más frías (B9). Este estudio permitió realizar nuevas estimaciones de los parámetros estelares y de los parámetros del viento. En tres objetos, la determinación de los parámetros del viento se ha realizado por primera vez. Analizamos la conocida relación momento-luminosidad del viento y discutimos nuestros resultados con los obtenidos por otros autores. Cabe destacar aquí que encontramos dos relaciones para describir la relación momento-luminosidad del viento de las estrellas supergigantes. Interpretamos estas relaciones en términos de los valores que adoptamos para los parámetros del viento.

Por otro lado, en esta tesis nos centramos en el estudio de la variabilidad de la supergigante $55 \mathrm{Cyg}$, sobre una base de tiempo de 5 años. Esto ha sido posible gracias a una extensa campaña de observación internacional. De un trabajo detallado hemos podido modelar con FASTWIND más de 50 espectros ópticos obteniendo de manera consistente los parámetros del viento. Complementariamente, el análisis de los momentos de líneas fotosféricas permitió detectar 19 frecuencias con periodos que van de unas pocas horas a varias decenas de días. La presencia de multiperiodos y la variabilidad observada en la eyección de materia nos llevó a proponer que 55 Cyg se encuentra por segunda vez en la región del diagrama HR de las supergigantes azules, habiendo pasado ya por la etapa de supergigante roja.

Discutimos además la relación del cociente entre la velocidad terminal y de escape con la temperatura efectiva de los objetos, prestando especial atención en aquellos que se encuentran en el rango de temperatura asociado con el salto de biestabilidad. Por otro lado, observando la variabilidad de los vientos estelares mediante los cambios en la forma e intensidad de los perfiles de línea de $\mathrm{H}_{\alpha}$, buscamos posibles correlaciones con los periodos reportados en la literatura. Encontramos una relación lineal empírica entre el máximo periodo (fotométrico o espectroscópico) medido y un parámetro $\mathrm{Q}_{\mathrm{r}}$ que está aso- 
ciado con la amplitud de variación de la pérdida de masa. Cabe mencionar que las estrellas supergigantes B que siguen esta relación son de tipos espectrales medios (B2-B3) y tardíos (B4-B9), mientras que las de tipos espectrales tempranos (B0-B1.5) parecen no seguir ninguna relación. La relación encontrada podría asociarse con los modos radiales extraños (que presentan los periodos más largos) y podrían desencadenar procesos de pérdida de masa. Por otro lado, cabe mencionar que el periodo máximo de 22.5 días y la amplitud de variación de la pérdida de masa encontrada en este trabajo para 55 Cyg, siguen la misma relación empírica mencionada.

En base a los resultados alcanzados en esta tesis, se plantean nuevas problemáticas a estudiar, como lo es la presencia de abundancias normales de He en la superficie de las estrellas supergigantes B en la etapa posterior a supergigante roja. Esto conlleva a rever los modelos de evolución estelar, indicando que estos no sólo deberían considerar la variabilidad en la pérdida de masa a lo largo de la evolución sino que deberían incluir a las pulsaciones en forma consistente.

El trabajo de tesis está diagramado en 6 capítulos; dos capítulos introductorios, un tercer capítulo donde se presenta una muestra de 19 estrellas B supergigantes, el modelado individual de cada una y un resumen de los parámetros estelares obtenidos y de las propiedades de los vientos de estas estrellas. El capítulo 4 versa sobre el estudio individual de la estrella supergigante 55 Cyg, donde se confirma la presencia de 19 modos de pulsación y se propone a las pulsaciones como un mecanismo plausible para explicar la variabilidad del viento en este objeto. En el capítulo 5 se presenta una discusión general sobre el estado evolutivo de las estrellas B supergigantes, las características de los vientos en la región de biestabilidad y se muestra una relación que vincula la amplitud de la variación de la pérdida de masa con las pulsaciones. Finalmente, en el capítulo 6 se resumen las conclusiones de este trabajo. 



\section{Índice general}

\begin{tabular}{|l|l}
\hline Agradecimientos & v
\end{tabular}

Resumen vii

$\begin{array}{ll}\text { 1. Introducción } & 1\end{array}$

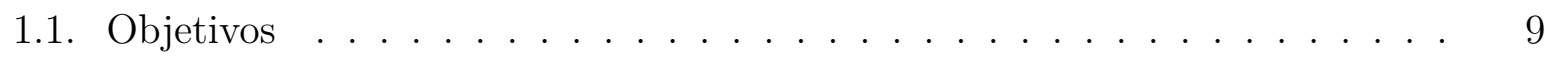

\begin{tabular}{ll}
\hline 2. Observación y Teoría & 11
\end{tabular}

$2.1 . \quad$ Breve reseña histórica $\ldots \ldots \ldots \ldots$. . . . . . . . . . . . . . . . 11

2.2. Efectos del viento estelar sobre las líneas espectrales . . . . . . . . . . . . . 12

2.2.1. Formación de un perfil P Cygni . . . . . . . . . . . . . . . . . . . . . . . . . . . . . . .

2.3. Parámetros del viento . . . . . . . . . . . . . . . . . . . . . . 17

2.4. Medición de los parámetros estelares . . . . . . . . . . . . . . . . . . 18

2.4.1. Temperatura efectiva $\left(\mathrm{T}_{\mathrm{ef}}\right) \ldots \ldots \ldots \ldots \ldots$. . . . . . . . . . . . . . . . . . . . . . . . . . . . . . .

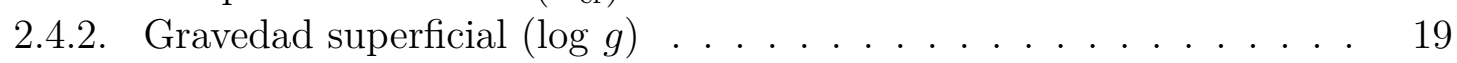

2.4.3. Radio $\left(\mathrm{R}_{\star}\right)$. . . . . . . . . . . . . . . . . . . . . . . . . . 19

2.4.4. Luminosidad y masa . . . . . . . . . . . . . . . . . . . . . 22

2.4.5. Velocidades de turbulencia y rotación . . . . . . . . . . . . . . . . . 22

2.4.6. Composición química . . . . . . . . . . . . . . . . . . . . 26

2.5. Teoría de vientos impulsados por radiación . . . . . . . . . . . . . . . 27

2.5.1. Ideas cualitativas . . . . . . . . . . . . . . . . . . 27

2.5.2. Ecuaciones básicas . . . . . . . . . . . . . . . . . . . . 29

2.5.3. Cálculo de la fuerza de radiación . . . . . . . . . . . . . . . . . . . . . . . . . . . . . . 31

2.5.4. Ecuación de movimiento . . . . . . . . . . . . . . . . . . 34

2.5.5. Relación momento-luminosidad del viento . . . . . . . . . . . . 36

2.6. Vientos inhomogéneos $\ldots \ldots \ldots \ldots$

2.6.1. Inestabilidades hidrodinámicas de los vientos impulsados por radiación 38

$2.6 .2 . \quad$ Clumping $\ldots \ldots \ldots \ldots \ldots \ldots \ldots$

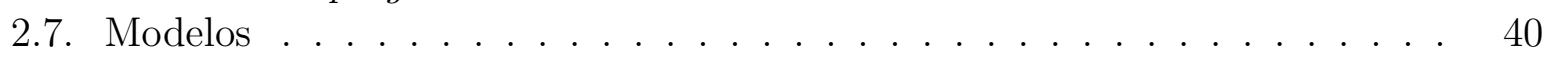

2.7.1. FASTWIND (Fast Analysis of STellar atmospheres with WINDs) . 41

2.8. BSGs: discrepancias con la teoría estándar . . . . . . . . . . . . . . . . 43 
2.9. Propiedades de los vientos estelares . . . . . . . . . . . . . . . . . 44

2.9.1. Salto de biestabilidad . . . . . . . . . . . . . . . 46

$2.9 .2 . \quad$ Variabilidad . . . . . . . . . . . . . . . . . 47

2.9.3. Posibles mecanismos de variabilidad . . . . . . . . . . . . 50

2.10. Mecanismos adicionales: pulsaciones . . . . . . . . . . . . . . 53

3. Análisis de una muestra de supergigantes B 59

3.1. Resumen . . . . . . . . . . . . . . . . . . . . . . . . . . 59

3.2. Observaciones . . . . . . . . . . . . . . . . . . . . . . 59

3.3. Determinación de parámetros estelares . . . . . . . . . . . . . . 60

3.4. Parámetros del viento . . . . . . . . . . . . . . . . . . . . . . . 63

3.5. Resultados . . . . . . . . . . . . . . . . . . . . . . 64

3.5.1. Comentarios individuales sobre cada objeto. . . . . . . . . . 65

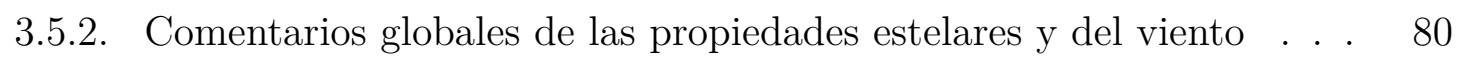

\begin{tabular}{ll}
\hline 4. El enigmático viento de 55 Cygni & 87
\end{tabular}

4.1. La estrella . . . . . . . . . . . . . . . . . . . . . . . . . . . . . 87

$4.2 . \quad$ Observaciones $\ldots \ldots \ldots \ldots$

$4.3 . \quad$ Resultados . . . . . . . . . . . . . . . . . . . . . . . . . . . . . . . . 90

4.3.1. Modelado de las líneas . . . . . . . . . . . . . . . . . . 90

$4.3 .2 . \quad$ Parámetros estelares . . . . . . . . . . . . . . . . . . . . 91

$4.3 .3 . \quad$ Variación del viento . . . . . . . . . . . . . . . . . . . . . 91

4.3.4. Velocidades de turbublencia y rotación . . . . . . . . . . . . . 92

$4.3 .5 . \quad$ Ajuste de perfiles $\ldots \ldots \ldots \ldots \ldots$

4.3.6. Errores . . . . . . . . . . . . . . . . . . . . . . . . . 101

$4.4 . \quad$ Discusión . . . . . . . . . . . . . . . . . . . . . . . . . 105

\begin{tabular}{ll}
\hline 5. Discusión general & 107
\end{tabular}

5.1. Parámetros estelares . . . . . . . . . . . . . . . . . . . . 107

5.2. Composición química . . . . . . . . . . . . . . . . . . . . . . 108

5.2.1. Abundancia de helio . . . . . . . . . . . . . . . . . . . . . . . 109

5.2 .2 . Abundancia de silicio . . . . . . . . . . . . . . . . . . 111

5.2 .3$. Abundancia de CNO . . . . . . . . . . . . . . . . 111

5.3. Estado evolutivo . . . . . . . . . . . . . . . . . . . . 117

5.4. Relación momento-luminosidad del viento (WLR) . . . . . . . . . . . . . 121

5.5. Salto de biestabilidad . . . . . . . . . . . . . . . . . . . . . . . . . 122

5.6. Pulsaciones y pérdida de masa . . . . . . . . . . . . . . . . 127

$\begin{array}{ll}\text { 6. Conclusiones } & 131\end{array}$

\begin{tabular}{ll}
\hline Apéndice A & 135
\end{tabular} 
\begin{tabular}{ll}
\hline Apéndice B & 145
\end{tabular}

6.1. Búsqueda de pulsaciones: análisis de momentos . . . . . . . . . . . . . . . 145

6.2. Análisis de frecuencias . . . . . . . . . . . . . . . . . . . . . 147 



\section{Capítulo 1}

\section{Introducción}

La radiación estelar se origina en los procesos nucleares que tienen lugar en el interior de las estrellas. Estos procesos producen cambios en la composición química interna y la luminosidad, ubicando a las estrellas en diferentes regiones del diagrama de HertzsprungRussell (HR). La evolución de las estrellas dependerá fundamentalmente de su masa inicial y su rotación, pero factores secundarios como la metalicidad, la eyección de materia o la interacción mutua de estrellas en sistemas binarios, pueden modificar radicalmente las fases evolutivas, y también las características del entorno estelar.

Las estrellas se clasifican en tres tipos de acuerdo a la masa que poseen. Iben \& Renzini (1984) propusieron clasificarlas en estrellas de baja masa $\left(<2-2.3 \mathrm{M}_{\odot}\right)$, masa intermedia (de 2-2.3 $\mathrm{M}_{\odot}$ a 8-9 $\mathrm{M}_{\odot}$ ) y estrellas masivas $\left(>8-9 \mathrm{M}_{\odot}\right)$. La variación en masa entre cada límite se debe a la composición química y momento angular iniciales de la estrella. El corte inferior de 2-2.3 $\mathrm{M}_{\odot}$ está ligado a cómo ocurre el encendido del He en el núcleo, que en el caso de las estrellas de baja masa, sucede en forma degenerada (debido a la presencia de electrones degenerados por las altas densidades), mientras que por encima de esas masas, la ignición del He se desarrolla de manera no degenerada. El límite superior, en cambio, está relacionado con el comportamiento del C-O en el núcleo estelar. Las estrellas de masa intermedia (que encienden el He central no degeneradamente) desarrollan un núcleo de C-O con electrones degenerados, mientras que las masivas lo desarrollan de forma no degenerada.

Las estrellas masivas a lo largo de sus fases evolutivas pueden llegar a perder más de la mitad de su masa debido a un flujo continuo de protones y electrones que escapa de la superficie de la estrella e inyecta materia al medio circunestelar. Este fenómeno se conoce como viento estelar. Dentro de estos objetos masivos se encuentran las estrellas supergigantes O (OSG), las supergigantes B (BSG) y las estrellas Wolf-Rayet (WR), cubriendo un rango de temperaturas efectivas entre $\sim 11000 \mathrm{~K}$ y $\sim 50000 \mathrm{~K}$ y radios estelares que pueden ir desde $10 \mathrm{R}_{\odot}$ a $50 \mathrm{R}_{\odot}$ (o incluso más), siendo así los objetos más brillantes de las galaxias.

Los diferentes estadíos evolutivos de las estrellas masivas pueden representarse mediante el diagrama de la fig. 1.1. Para los objetos extremadamente masivos, que superan las $50 / 60 \mathrm{M}_{\odot}$, los modelos evolutivos predicen que en la secuencia principal sus vientos son lo suficientemente fuertes como para expulsar las capas más externas de la atmósfera, dejando 


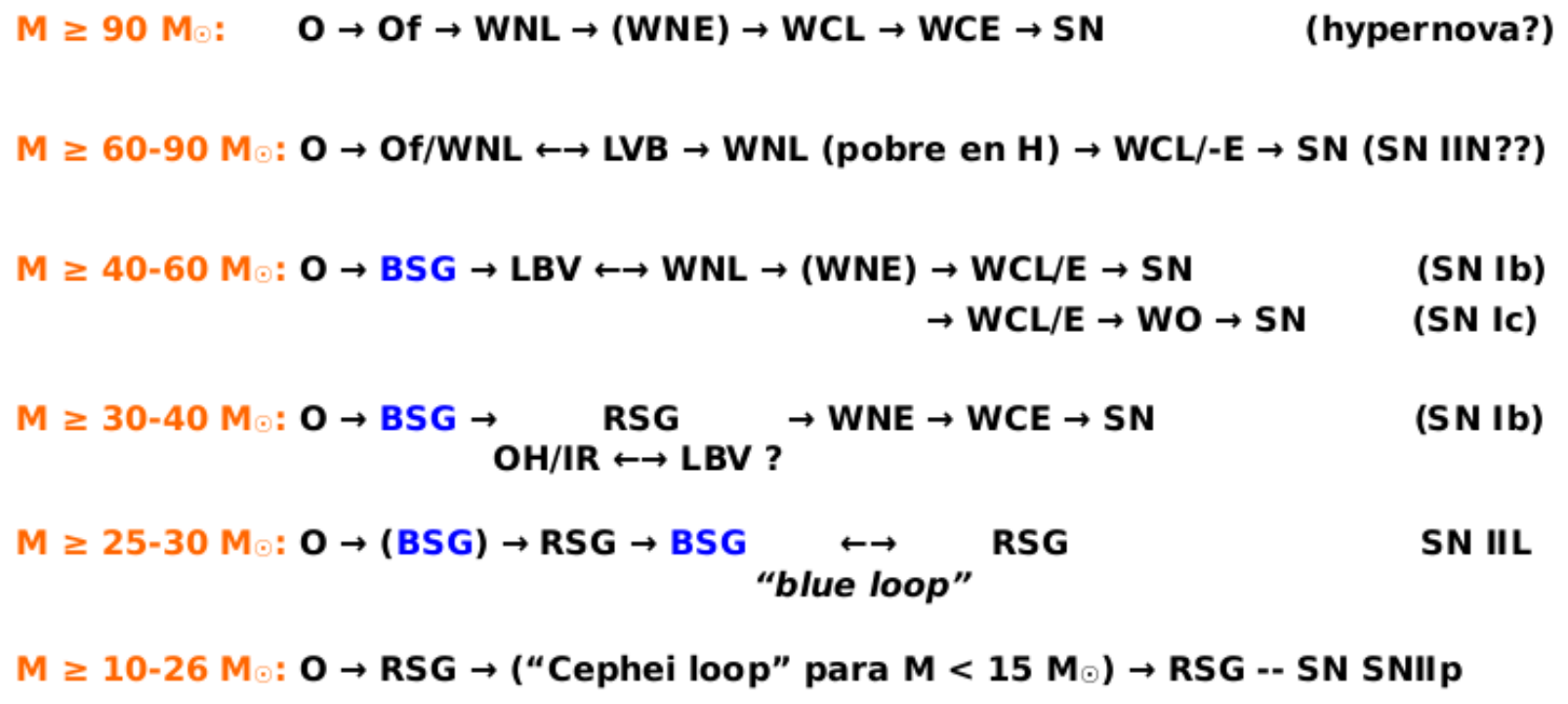

Figura 1.1. Diferentes fases evolutivas de estrellas masivas con metalicidad solar y pérdida de masa. Todos los estadíos que empiezan con la letra W corresponden a la fase de Wolf-Rayet y la siguiente letra está asociada con la principal abundancia de CNO, mientras que las letras L y E denotan la presencia o pérdida de la envoltura más externa de $\mathrm{H}$, respectivamente. Las otras siglas denotan: LBV, variable azul luminosa; BSG, supergigante azul; RSG, supergigante roja; SN I, supernova sin H; SN II supernova que muestra H; SN Ib/c, supernova con absorción débil o nula de silicio (b muestra la línea de HeI a $587.6 \mathrm{~nm}$ mientras que en c, es débil o nula); SN II-p/L, supernova que no presenta líneas delgadas y la curva de luz de L decae en forma lineal, mientras que en la p es no lineal o tipo "plateau"; SN IIn, supernova que presenta algunas líneas delgadas. Estrellas $\mathrm{OH} / \mathrm{IR}$ son objetos de la rama asintótica de las gigantes con fuertes emisiones máser de $\mathrm{OH}$. Las flechas indican un posible sentido evolutivo. "Blue loop" hace referencia a más de un pasaje por la etapa de supergigante azul, mientras que "cephei loop" está asociado con una etapa evolutiva de cefeida. Extraído de Maeder \& Meynet 2008. 
al descubierto capas con elementos pesados que, previamente, formaban parte del núcleo de la estrella. Es por este motivo que los objetos con estas características no evolucionan hacia la fase de supergigante roja (RSG) y siempre permanecen en la parte azul del diagrama HR.

En las estrellas con masas iniciales intermedias $\left(25 \mathrm{M}_{\odot} \leq \mathrm{M} \leq 40 \mathrm{M}_{\odot}\right)$, los vientos en las fases de secuencia principal y supergigante azul no son lo suficientemente intensos como para eyectar las capas externas. Se supone que estos objetos alcanzan rápidamente la fase de RSG, donde los vientos progresivamente desprenden sus envolturas. Cuando el núcleo de He alcanza un tamaño crítico respecto a la masa total (que decrece), la estrella vuelve a la fase de BSG. Este pasaje evolutivo se conoce como "blue loop" y la fase de BSG dura muy poco tiempo respecto a la fase de RSG. Luego, la estrella se convertirá en una WR y continuará su evolución.

Si los objetos poseen una masa inicial menor a $25 \mathrm{M}_{\odot}$, al abandonar la secuencia principal pasan a la fase de RSG y vuelven hacia la parte azul del diagrama HR, pasando probablemente por la fase de Cefeida ("Cephei loop" en la fig. 1.1), y luego regresan a la fase de RSG para finalizar como supernova (SN). En las fases BSG y RSG los vientos no alcanzan a desprender las capas externas.

Estudios más realistas a partir de modelos con rotación realizados por Ekström et al. (2012), demostraron que si las masas iniciales son mayores que $15 \mathrm{M}_{\odot}-20 \mathrm{M}_{\odot}$, las estrellas pierden gran parte de su envoltura externa de hidrógeno en la fase de supergigante roja y evolucionan nuevamente hacia la parte azul del diagrama HR (ver fig. 1.2). La expulsión de las capas más externas de la estrella en la fase de RSG requiere que la tasa de pérdida de masa sea, al menos, un factor 3 mayor que la tasa de pérdida de masa en la fase de BSG Ekström et al. 2012 Saio et al.2013a. Este evento, en conjunto con el "dragado" o procesos de mezcla, dejarían al descubierto en la superficie estelar elementos pesados ya procesados, como el C, N y O.

Del esquema anterior y de la fig. 1.2. podemos encontrar que los modelos con masas iniciales de $\sim 20 \mathrm{M}_{\odot} \mathrm{a} \sim 40 \mathrm{M}_{\odot}$ sugieren la coexistencia de dos poblaciones de objetos con características evolutivas muy diferentes: una población que recién sale de la secuencia principal, y otra que ya evolucionó a la fase de supergigante roja y está, por segunda vez, en la región azul del diagrama HR. Por lo tanto, una manera de poder distinguir entre ambas poblaciones de BSGs sería mediante la determinación de abundancias químicas. Sin embargo, la mayoría de las observaciones no concuerdan con los modelos evolutivos en lo que respecta a abundancias, ya que estos últimos predicen un valor mayor de la abundancia de CNO superficial que el que se observa Takeda \& Takada-Hidai 2000 Przybilla et al. 2006, Saio et al. 2013a.

Existen varios modelos de evolución de estrellas masivas, como por ejemplo, los correspondientes a los grupos de Ginebra Ekström et al. 2012) y Padova (Bertelli et al. 2009), FRANEC Chieffi \& Limongi 2013), STAREVOL (Decressin et al. 2009), STERN (Brott et al.2011) y MESA (Paxton et al. 2011, 2013). Sin embargo, Martins \& Palacios 2013). encuentran que todos los modelos coinciden en reproducir bien la fase de secuencia principal, pero durante la evolución de post-secuencia principal presentan grandes diferencias en luminosidad y temperatura, como muestra la fig. 1.3. Esto conduce a una gran incer- 


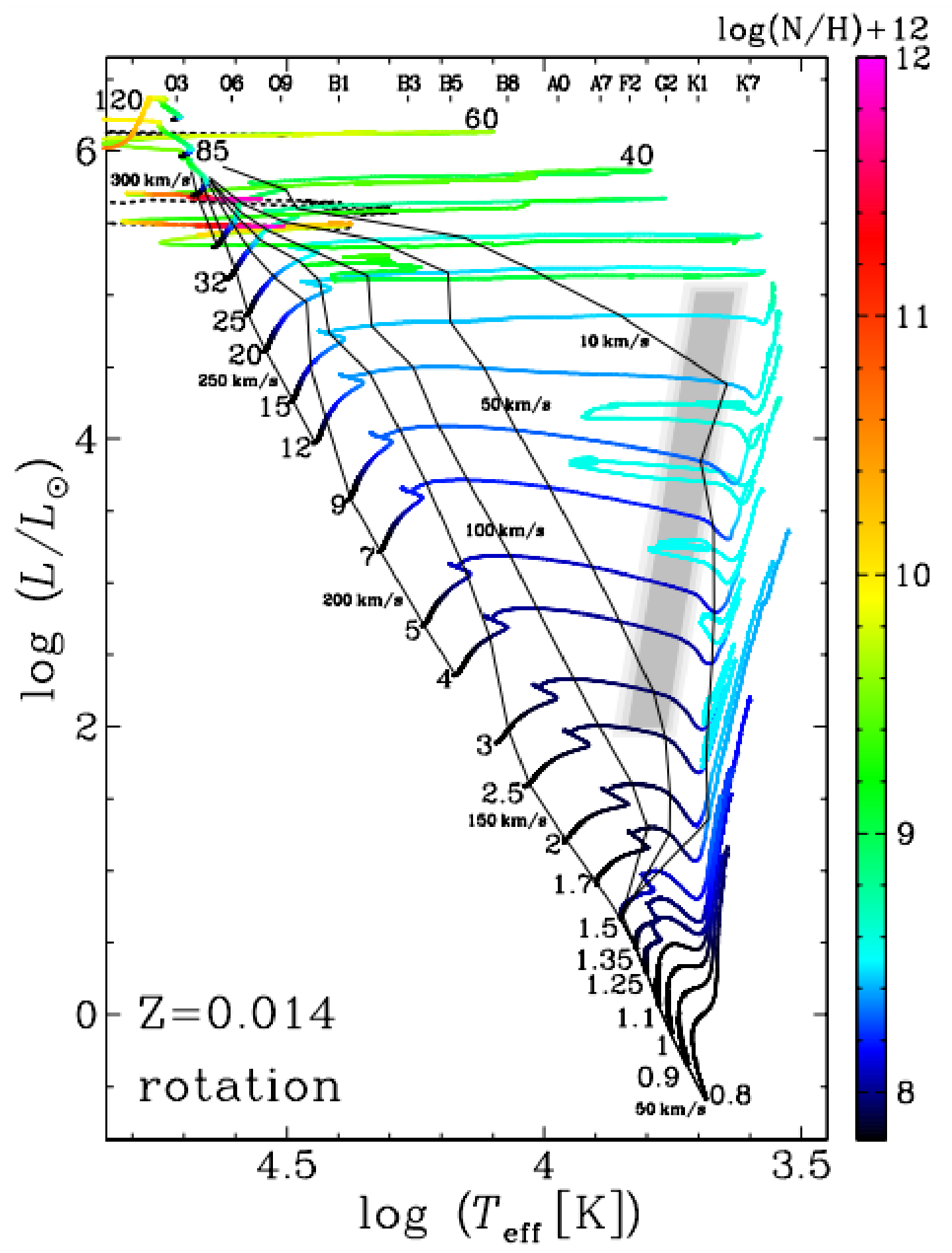

Figura 1.2. Diagrama HR para modelos evolutivos con masas iniciales entre $0.8 \mathrm{M}_{\odot}$ y 120 $\mathrm{M}_{\odot}$ con rotación. La escala de colores indica la abundancia superficial de nitrógeno predicha. Extraído de Ekström et al. 2012. 

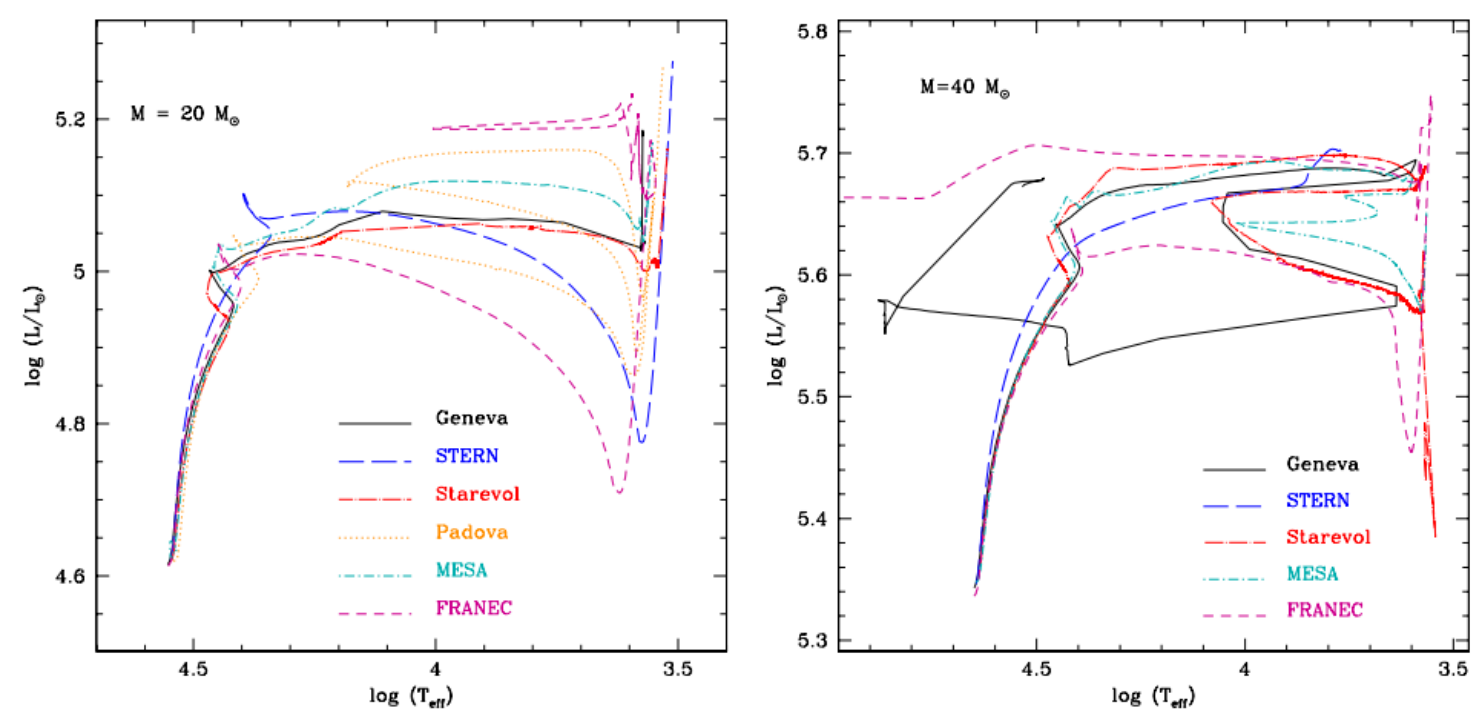

Figura 1.3. Diagrama de Hertzsprung-Russell (HR) con diferentes modelos evolutivos (curvas de diferente color para cada modelo) para masas iniciales de $20 \mathrm{M}_{\odot}$ (derecha) y $40 \mathrm{M}_{\odot}$ (izquierda) Martins \& Palacios 2013

teza en la determinación de los valores para las masas estelares. Las diferencias entre los caminos evolutivos obtenidos por los diferentes códigos se deben a los ingredientes físicos adoptados en cada uno, como por ejemplo, la abundancia solar, la metalicidad, parámetro de overshooting Đ la tasa de pérdida de masa, entre otros.

El final de una estrella masiva ocurre con el colapso del núcleo y explosión como supernova. Se espera que los objetos con masas iniciales entre $8 \mathrm{M}_{\odot}$ y $20 \mathrm{M}_{\odot}$ retengan su envoltura externa de $\mathrm{H}$ y exploten en la fase de RSG, resultado que fue confirmado por pre-explosiones detectadas en estrellas progenitoras de SN II $2^{2}$ Langer 2012. Sin embargo, investigaciones sobre la SN 1987A plantean un panorama alternativo, ya que el objeto observado fue una SN de tipo II generada por una BSG con masa inicial de $18-20 \mathrm{M}_{\odot} \mathrm{y}$ no por una RSG (Smith et al. 2013, y sus referencias).

Evidencias de fuertes eyecciones de masa en una fase previa a RSG fueron reportadas también por Mahy et al. (2016). Según estos autores, el instante de eyección de materia de la candidata a LBV HD 168625 (B6Iap) (fig. 1.4 está restringido a dos momentos evolutivos: durante o justo después de la fase de BSG y antes de la fase de RSG, sin haber pasado por esta última fase. Esta conclusión fue obtenida mediante espectroscopía e imágenes infrarrojas en conjunto con mediciones de abundancias de CNO. Situaciones similares fueron reportadas también en otros objetos como Sher 25 (B1.5lab; Smartt et al. 2002 y SWB1 (B1.5 Iab; Smith et al. 2007.

Por lo tanto, el comportamiento y entendimiento de los caminos evolutivos de las estrellas masivas no son completos Martins \& Palacios 2013, ya que algunas fases evolutivas,

\footnotetext{
${ }^{1}$ Este parámetro es una medida que determina la extensión de la zona convectiva.

${ }^{2}$ Estos objetos son el subgrupo más grande de supernovas.
} 


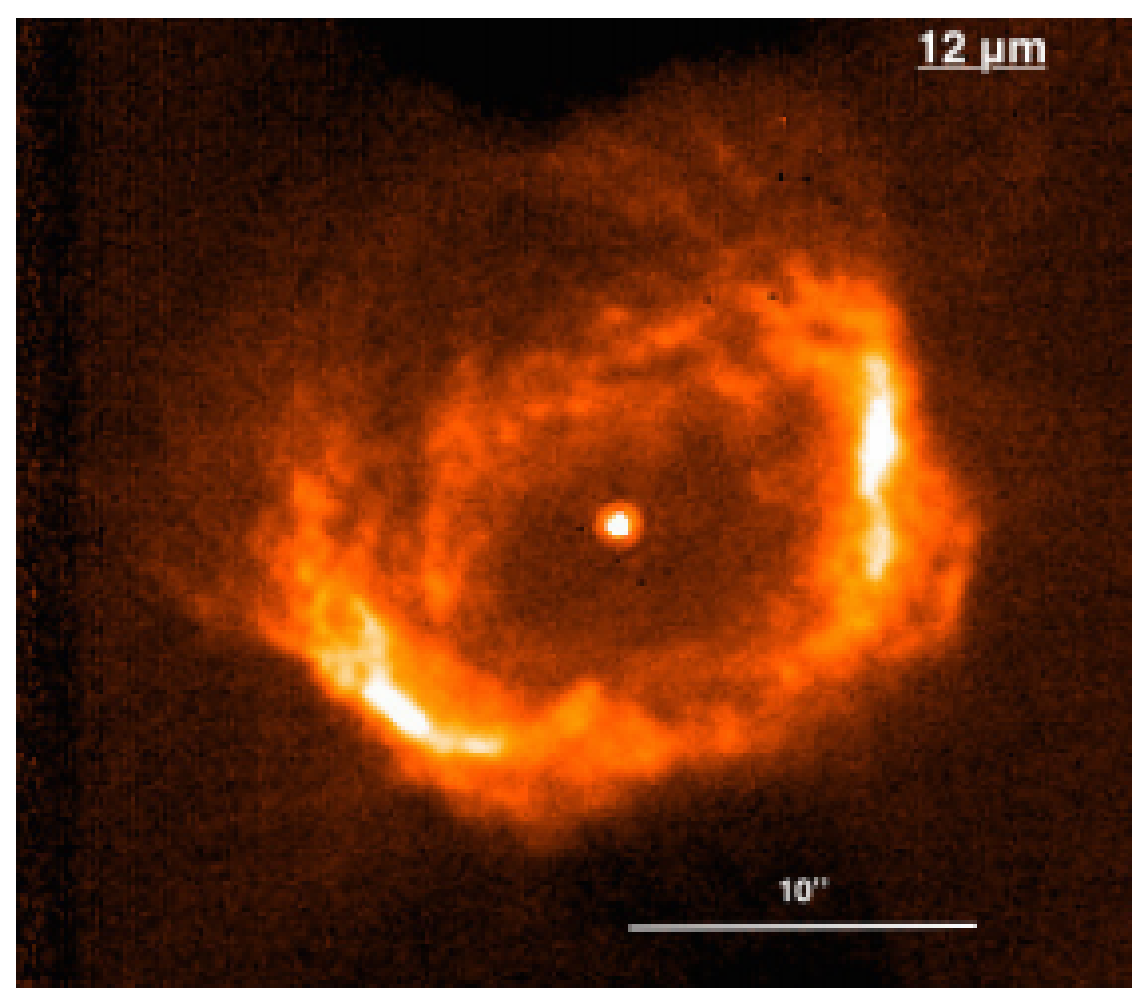

Figura 1.4. Nebulosa alrededor de HD 168625 . Imagen en $12 \mu \mathrm{m}$ de VLT/VISIR Mahy et al. 2016. 


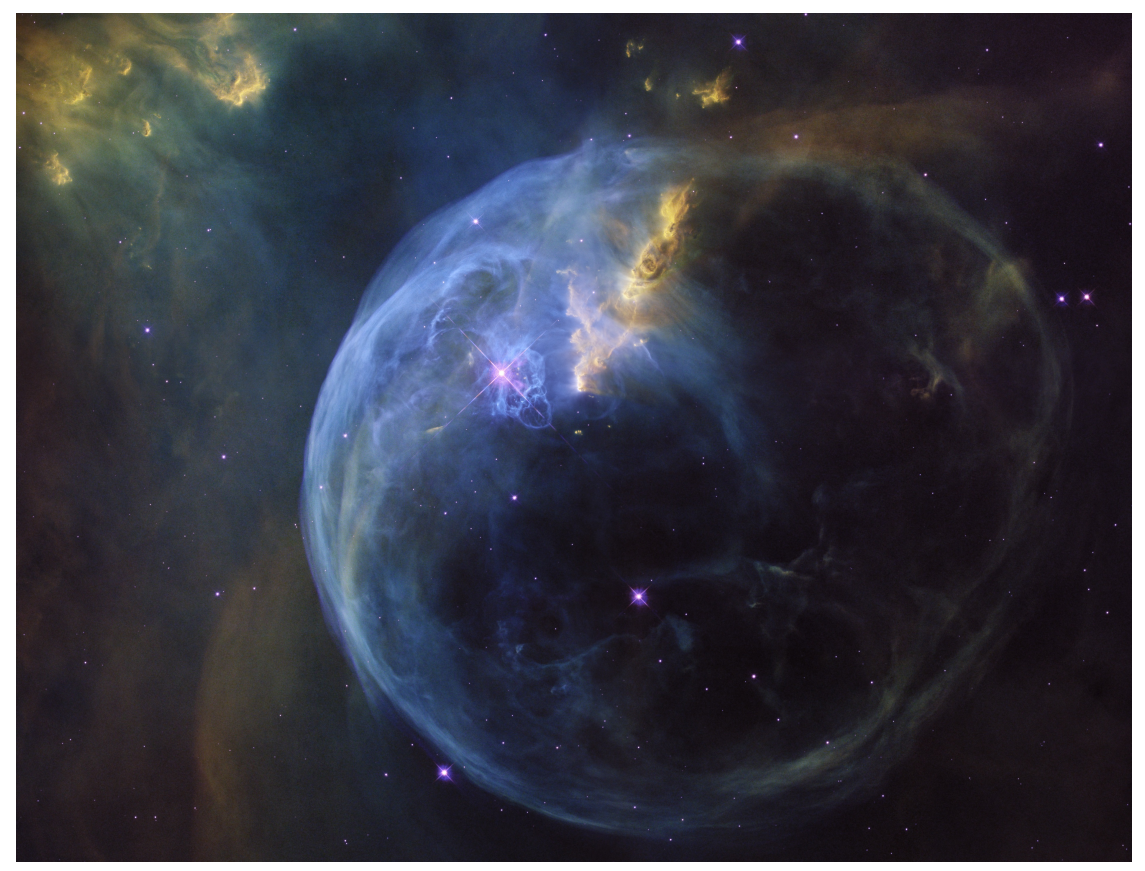

Figura 1.5. NGC 7635 es uno de los tantos ejemplos de cómo los vientos estelares son capaces de generar estas grandes burbujas interestelares. Créditos: NASA, ESA, Hubble Heritage Team.

como LBV, supergiantes $\mathrm{B}[\mathrm{e}]^{3}$ o los detalles de las fases finales, no han sido reproducidas o explicadas correctamente con los modelos. Así, podemos ver que en lo que concierne a la evolución de estrellas masivas aún quedan por descrifrar varios interrogantes, por lo que es muy importante tener mediciones precisas de los parámetros estelares fundamentales y de la pérdida de masa ya que todos ellos forman parte de las diferentes propiedades involucradas en los modelos.

Es importante además destacar que las estrellas masivas desempeñan un papel crucial en la evolución química y dinámica del gas de las galaxias, a través de la materia que inyectan en forma de viento y de explosión de supernova. En particular, los vientos estelares no sólo modifican al objeto en cuestión sino que también modifican su entorno. Las partículas que se desprenden de las estrellas transfieren energía y momento a las partículas del medio interestelar circundante dando lugar a la formación de grandes burbujas o cavidades interestelares, como puede apreciarse en la fig. 1.5. De esta manera, también se inyecta al medio materia procesada por la estrella a medida que ésta evoluciona, enriqueciendo con elementos pesados sus alrededores. Adicionalmente, los fuertes vientos también favorecen a la tasa de nacimiento de nuevas estrellas debido a la compresión de la materia circundante. Por estas razones a las estrellas masivas se las suelen llamar los "motores" de la evolución química y dinámica de las galaxias espirales.

\footnotetext{
${ }^{3}$ Supergigantes de tipo espectral B con líneas prohibidas en emisión y fuertes excesos infrarrojos que indican la presencia de discos de gas y polvo.
} 
Como mencionamos anteriormente, dentro de las estrellas masivas podemos encontrar a las estrellas supergigantes de tipos espectrales O y B, y a las estrellas Wolf-Rayet, como objetos que ya evolucionaron de la secuencia principal y que presentan los vientos estelares más intensos medidos. Esto se ve reflejado en que los valores observados para las tasas de pérdida de masa son del orden de $10^{-7} \mathrm{M}_{\odot} /$ año a $10^{-4} \mathrm{M}_{\odot} / \mathrm{añ} \coprod^{4}$

Los vientos de estas estrellas pueden explicarse mediante la teoría de vientos impulsados por radiación Castor et al. 1975, Pauldrach et al. 1986, cuya idea primordial está basada en el hecho de que un átomo o ion puede adquirir impulso mediante el proceso de absorción de un fotón. Esta teoría, conocida como teoría estándar, propone que la tasa de pérdida de masa y la velocidad máxima que alcanzan las partículas del viento (velocidad terminal) deben ser constantes. La solución hidrodinámica del viento se puede aproximar empleando una ley de velocidades (ver cap. 22 cuyo exponente $\beta$ toma valores entre 0.5 y 0.8 Castor et al. 1975 Abbott 1982. Por otro lado, la teoría estándar predice una de las relaciones más importantes que es la relación momento-luminosidad del viento, a partir de la cual pueden determinarse las distancias de los objetos con sólo determinar los parámetros del viento. Las predicciones de esta teoría concuerdan muy bien con las observaciones de las estrellas más calientes (O, B0 y B1), mientras que para las estrellas supergigantes B1.5B9 se observan ciertos apartamientos. Por ejemplo, las relaciones momento-luminosidad observadas para estos objetos caen sistemáticamente por debajo de la relación teórica Crowther et al. 2006a Searle et al. 2008a y los valores de $\beta$ observados se encuentran entre 1 y 3 (Crowther et al. 2006a; Markova et al.2008a.

Las estrellas BSGs presentan variaciones tanto en sus curvas de luz como en sus líneas espectrales (Morel et al. 2004, Lefever et al. 2007), pero las variaciones más intensas están presentes en la línea $\mathrm{H}_{\alpha}$, que cambia tanto su forma como intensidad. Esta línea puede desplegar perfiles P Cygni, perfiles en emisión o absorción puras, e incluso se han reportado observaciones donde el perfil desaparece por completo Morel et al. 2004, Maharramov 2013. La línea $\mathrm{H}_{\alpha}$ es la línea más sensible, en el rango óptico, a las propiedades de los vientos estelares (Puls et al. 2008). Por lo tanto, las variaciones observadas en esta línea sugieren la presencia de cambios en la estructura de los vientos de estas estrellas, por lo que la tasa de pérdida de masa y la velocidad terminal del viento ya no serían parámetros constantes. Esto lleva a preguntarse: ¿Cuál es el origen de la variabilidad observada en los perfiles de líneas espectrales de estas estrellas? ¿Qué cantidad de masa pierden las estrellas en las diferentes fases de su vida? ¿Cómo es la estructura de los vientos en las estrellas masivas? ¿Existen otros mecanismos, que junto con la fuerza de radiación, sean capaces de impulsar el viento y desencadenar una pérdida de masa variable?

Los mecanismos que favorecen la eyección de materia pueden ser la rotación, la presión de radiación y, en especial, las explosiones de supernovas. Aparte de estos mecanismos, existe en particular uno, al cual varios autores proponen como el responsable de las variaciones de las curvas de luz y de las líneas espectrales fotosféricas, que son las pulsaciones Aerts et al.2009a Simón-Díaz et al. 2010. Estudios teóricos predicen que las dos subpoblaciones BSG del diagrama HR tendrían características pulsacionales diferentes, sugiriendo la pre-

\footnotetext{
${ }^{4}$ Estas cantidades son mucho mayores a la tasa de pérdida de masa del Sol que es de $10^{-14} \mathrm{M}_{\odot}$ año ${ }^{-1}$.
} 
sencia de modos radiales extraños en aquellas estrellas que ya evolucionaron de la fase de RSG Saio et al. 2013b). De esta manera, podríamos preguntarnos si las pulsaciones también serían responsables de las variaciones observadas en los vientos y si, además, pueden afectar la tasa de pérdida de masa.

\subsection{Objetivos}

Las estrellas supergigantes siempre han sido consideradas como objetos claves en la evolución estelar ya que éstas brindan un escenario adecuado para discutir la influencia de los vientos en las diferentes fases de la evolución estelar. Para realizar este estudio nos proponemos investigar 20 estrellas B supergigantes, empleando observaciones espectroscópicas adquiridas en alta resolución en el rango óptico que nos permitan:

- Determinar parámetros estelares fundamentales y la composición química superficial para precisar el estado evolutivo de estos objetos.

- Determinar las propiedades del viento estelar.

- Discutir la relación momento-luminosidad del viento.

- Analizar la variabilidad del viento.

- Vincular el origen y propiedades del viento con diferentes modos y períodos de pulsación.

El entendimiento de estas propiedades y fenómenos tendrá impacto en el estudio de poblaciones estelares, evolución estelar, brotes de formación estelar, supernovas, nucleosíntesis, producción de polvo y cosmología. 



\section{Capítulo 2}

\section{Observación y Teoría}

Para entender la evolución de las estrellas masivas y los ciclos por los que éstas transitan a lo largo de su vida, es necesario conocer su posición en el diagrama HR e investigar los procesos físicos que podrían estar presentes. En particular nos interesa estudiar las propiedades de los vientos estelares de las estrellas supergigantes B (BSGs), por lo que, en la primera parte de este capítulo haremos una breve reseña histórica acerca del estudio de este fenómeno. También describiremos las características y la formación de los perfiles de líneas cuando éstos se ven afectados por la presencia de un viento estelar.

Luego explicaremos los métodos y herramientas para determinar los parámetros estelares y los parámetros del viento. Es decir, de qué manera es posible estimar la temperatura efectiva $\left(T_{e f}\right)$, la gravedad superficial (log g), el radio $\left(R_{\star}\right)$, la luminosidad $(L)$, la masa $(M)$, la composición química, la velocidad de microturbulencia $\left(\mathrm{v}_{\mathrm{mic}}\right)$, la velocidad de macroturbulencia $\left(\mathrm{v}_{\mathrm{mac}}\right)$, la velocidad de rotación proyectada ( $\mathrm{v}$ sen $\mathrm{i}$ ), la tasa de pérdida de masa $(\dot{\mathrm{M}})$, la velocidad terminal $\left(\mathrm{v}_{\infty}\right)$ y el campo de velocidades (medido a través del parámetro $\beta$, ver secc. 2.7.1.

Por último, desarrollaremos las ideas cualitativas y ecuaciones básicas de la teoría que explica los vientos estelares de las estrellas masivas y también describiremos las principales características del código computacional que usaremos en este trabajo, para finalmente abordar las características observacionales de las estrellas BSGs.

\subsection{Breve reseña histórica}

Los primeros indicios de pérdida de masa estelar se remotan a mediados del s. XVI, en 1572, cuando Tycho Brah円 aumento repentino de su brillo, que superó la magnitud -4 y que hizo que este objeto sea visible durante el día, fue disminuyendo lentamente en los siguientes 16 meses. Esto llevó a Brahe a asociar este fenómeno eruptivo con una gran explosión. Variaciones significativas de brillo también fueron observadas 28 años más tarde por Joan Blaed ${ }^{2}$ pero en la estrella $\mathrm{P}$

\footnotetext{
${ }^{1} 14 / 12 / 1546-24 / 10 / 1601$.

$223 / 09 / 1596-28 / 05 / 1673$.
} 
Cygni, que aumentó 3 magnitudes su brillo y mostró durante los siguientes 6 años grandes variaciones de luz, para luego permanecer casi constante con una magnitud de $\sim 5$.

Recién en el s. XIX, con el desarrollo de la espectroscopía, se descubren varios objetos (novas y estrellas Wolf Rayet) cuyas líneas espectrales eran similares a las que mostraba la estrella P Cygn ${ }^{3}$ (motivo por el cual este tipo de perfiles se conocen como perfiles P Cygni). $\mathrm{Si}$ bien las primeras interpretaciones de estos perfiles se atribuyeron al efecto Doppler, no quedaba claro si era producido por una expansión o era un fenómeno asociado a algún tipo de turbulencia. Dicha discusión queda totalmente clarificada cuando se emplearon, conjuntamente con la espectroscopía, técnicas fotográficas que revelaron en las novas la presencia de cáscaras de materia o "shells" centrados en el lugar de las explosiones y que aumentaban su diámetro con el tiempo. Con la observación directa de la Nova Aurigae, se concluye que la presencia de perfiles tipo P Cygni está asociada a la expansión de las envolturas gaseosas de las estrellas.

\subsection{Efectos del viento estelar sobre las líneas espec- trales}

Los indicadores más sensibles a la pérdida de masa de una estrella caliente son las líneas espectrales producidas por transiciones atómicas del estado fundamental al primer estado excitado de los iones más abundantes, también llamadas líneas de resonancia.

Si el viento es denso, es decir, los iones absorbentes tienen una densidad columnar elevada (más de $10^{15}$ partículas $/ \mathrm{cm}^{2}$ ), las líneas espectrales comienzan a presentar una forma muy particular, la que se denomina perfil "P Cygni". Este perfil está caracterizado por una componente en absorción, desplazada hacia longitudes de onda cortas, y una componente en emisión, centrada en la longitud de onda en reposo y está afectada por la componente en absorción (fig. 2.1).

En general, la mayoría de los perfiles P Cygni de las supergigantes O y B se observan en las líneas de resonancia en la región UV del espectro de elementos altamente ionizados (por ejemplo, C IV, Si IV, N V y HeII). Este proceso se caracteriza por la absorción de un fotón seguida por una fotodesexcitación donde el fotón es emitido en otra dirección. Este mecanismo se conoce como "scattering" de fotones. La primera determinación de pérdida de masa basada en el modelado de los perfiles P Cygni fue realizada por Lamers \& Morton 1976.

Las estrellas con altas tasas de pérdida de masa (del orden de $10^{-6} \mathrm{M}_{\odot} /$ año o más) también pueden presentar sus líneas en emisión pura (fig. 2.2 en la región del espectro óptico, las cuales también proveen información sobre la tasa de pérdida de masa y la velocidad del viento.

La mayoría de las líneas de emisión se forman gracias a la captura electrónica de los iones. El electrón es capturado a algún estado excitado del ion, para luego decaer por

\footnotetext{
${ }^{3}$ Estas líneas se caracterizan por tener una componente en absorción desplazada hacia el azul, y una componente en emisión centrada en la longitud de onda en reposo.
} 


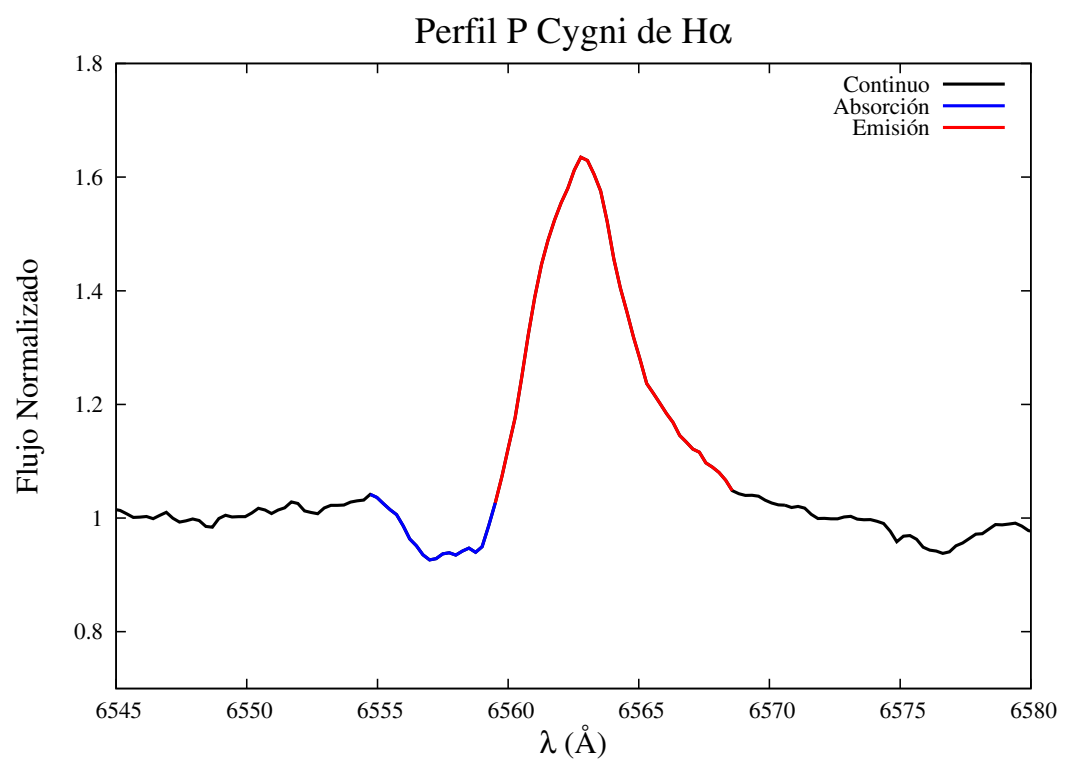

Figura 2.1. Perfil P Cygni de la línea $\mathrm{H}_{\alpha}$ de HD 148688 (B1Iae). Negro, azul y rojo hacen referencia al continuo, componente en absorción y componente en emisión, respectivamente. Espectro adquirido con el espectrógrafo REOSC montado en el telescopio "Jorge Sahade" $2.15 \mathrm{~m}$ de CASLEO.

cascada y dar lugar a la emisión de fotones (fotodesexcitación). Este proceso completo se conoce como recombinación y los ejemplos más conocidos de líneas en el rango óptico son las líneas $\mathrm{H} \alpha(\lambda=6562 \AA)$ y He II $(\lambda=4686 \AA)$. La primera determinación de pérdida de masa a partir de la emisión de la línea de $\mathrm{H} \alpha$ fue hecha por Klein \& Castor 1978) en estrellas $\mathrm{O}$ f El estudio del viento estelar mediante la línea $\mathrm{H} \alpha$ tiene la gran ventaja que ésta puede ser observada desde Tierra, en contraste con los perfiles P Cygni UV que requieren observaciones satelitales.

\subsubsection{Formación de un perfil P Cygni}

Supongamos un modelo simple de viento estelar con simetría esférica, donde la velocidad de las partículas aumenta a medida que éstas se alejan de la estrella; es decir, un viento acelerado. La fig. 2.3 ilustra esta situación, donde las flechas negras indican la dirección e intensidad del campo de velocidades. Un observador ubicado a la izquierda de la estrella puede detectar 4 regiones:

1. La estrella, que emite un flujo continuo de fotones,

2. la región del viento proyectada por delante de la estrella, donde el gas se mueve con velocidades entre $0 \mathrm{~km} / \mathrm{s}$ (A) y $\mathrm{v}_{\mathrm{m}}$ (máxima, A') hacia el observador,

\footnotetext{
${ }^{4}$ Estrellas de tipo espectral O que presentan la línea He II $\lambda 4686$ en emisión.
} 


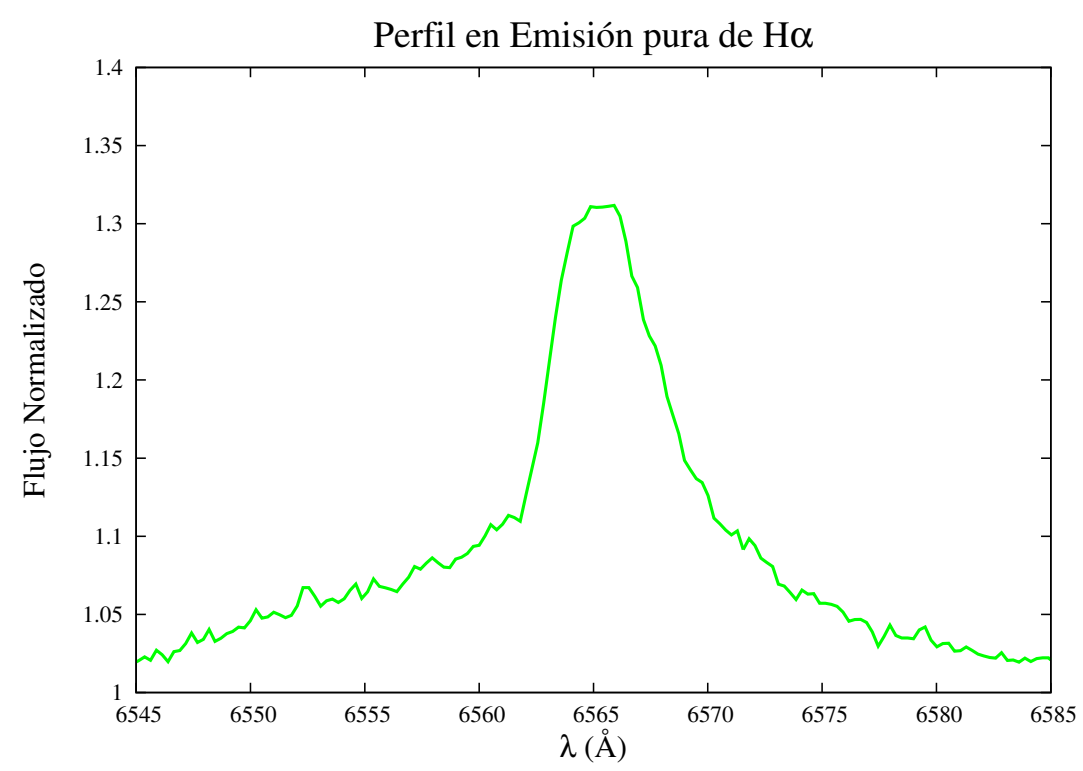

Figura 2.2. Perfil de línea de $\mathrm{H}_{\alpha}$ observada en emisión pura de $\alpha$ Cam (O9I). Espectro adquirido con el espectrógrafo de ranura larga montado en el telescopio $1.5 \mathrm{~m}$ de Tartu Observatory, Estonia. Crédito: Dra. Michaela Kraus y Dra. Anna Aret.

3. la región ubicada detrás de la estrella, según el observador, análoga a la región anterior, de la cual, en principio, no le llega información, y

4. las regiones laterales o "halo", donde el gas se expande radialmente, y la proyección de dichas velocidades respecto al observador externo toman valores positivos (B) y negativos $(\mathrm{C}) \mathrm{5}^{5}$

Analizamos ahora cada una de las correspondientes regiones del viento:

- Absorción: región $1+2$. Los fotones provenientes de la estrella son dispersados en una dirección distinta a la del observador produciendo una línea de absorción pura con respecto al continuo de la estrella. Este continuo está caracterizado por una línea horizontal en "1" (ver parte inferior de la fig. 2.3). Una transición electrónica ocurre si el fotón posee una energía compatible con la energía de separación entre dos niveles del ion absorbente, en su propio marco de referencia. Pero, dado que la materia está acelerándose, por efecto Doppler, el observador detectará fotones con frecuencias $\nu$ mayores comparadas con la frecuencia en reposo. Por lo tanto, en la parte del continuo en un medio en movimiento con expansión radial y cuya velocidad aumenta a medida que se aleja de la estrella (desde $0 \mathrm{~km} / \mathrm{s} \mathrm{a}_{\mathrm{m}}$ ), tendremos una absorción cuyo rango espectral estará desplazado entre $\mathrm{v}_{\mathrm{m}}$ y $0 \mathrm{~km} / \mathrm{s}$, influyendo directamente en la forma del perfil de absorción que se observa (fig. 2.3. abajo). Este proceso de absorción

\footnotetext{
${ }^{5}$ Consideramos positivos si se acercan al observador y negativos si se alejan.
} 


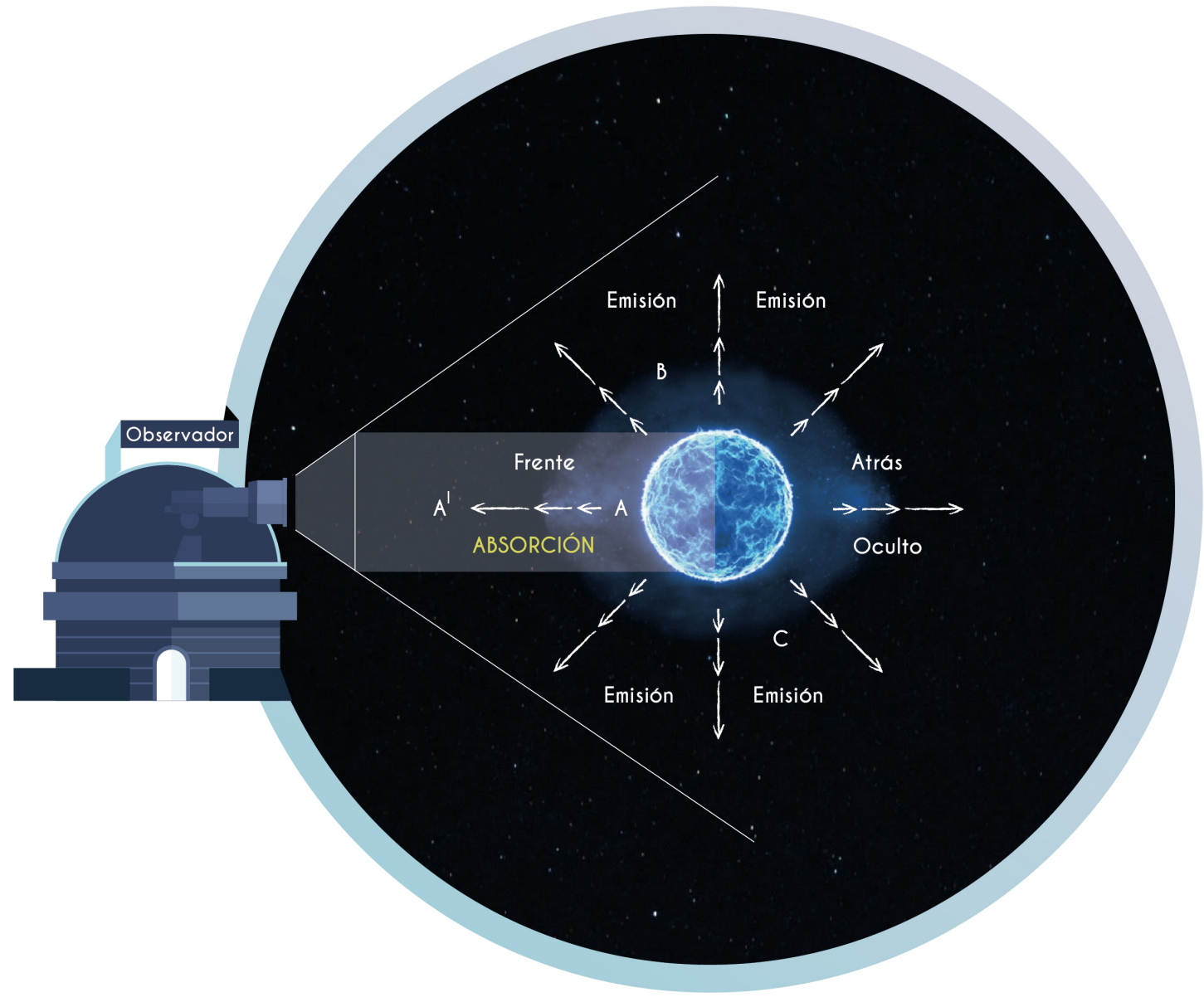

Absorción

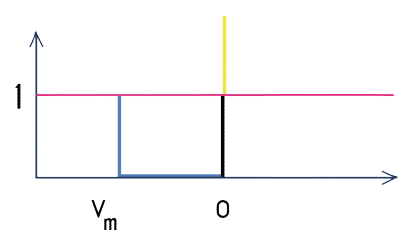

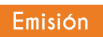

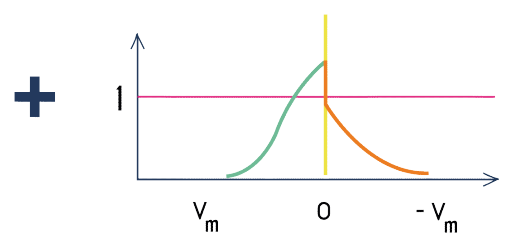

Perfil P Cygni

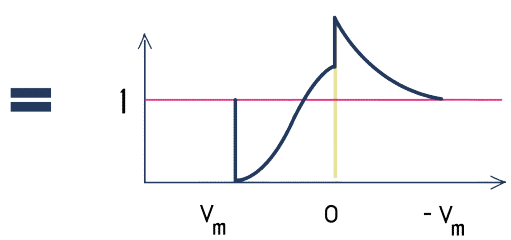

Figura 2.3. Imagen ilustrativa sobre la formación de un perfil P Cygni. En la parte superior se muestran las diferentes zonas detalladas en el texto, mientras que en la parte inferior del gráfico se muestra la combinación resultante de los perfiles de absorción y emisión, originando así un perfil P Cygni. Diseñado y realizado por la Lic. Daniela Haucke. 
ocurre en la parte A del viento, en frente del disco estelar. Notemos que a ambos lados del perfil de absorción el observador ve el continuo estelar sin atenuaciones, ya que no hay materia presente que pueda absorber esta radiación en el rango de frecuencias correspondiente.

- Emisión: región 4 (halo) $+2+3$. Debido al corto lapso de tiempo que dura un estado excitado, un nuevo fotón es reemitido después de la absorción y el observador ve en conjunto un perfil de emisión que resulta de una multitud de estos procesos a diferentes velocidades. Como la emisión del viento provee radiación adicional, el correspondiente perfil comienza en cero (0) y no en uno (1). En contraste con la absorción, hay varias regiones que contribuyen a la componente en emisión. La región 2 , en frente al disco estelar, es decir, la parte que absorbe, es una de ellas. Teniendo que cuenta que los procesos de emisión son (casi) isotrópicos, una cierta fracción siempre será reemitida en la dirección del observador. Por otro lado, el material del halo también contribuye. Los fotones provenientes de la fotosfera serán dispersados en diferentes direcciones, y algunas de estas direcciones coincidirán con la dirección del observador, produciendo una emisión (simplemente por ser un gas con una temperatura determinada) con un ancho Doppler apenas menor al rango $\left[\mathrm{v}_{\mathrm{m}} ;-\mathrm{v}_{\mathrm{m}}\right.$ ] (ya que la velocidad máxima proyectada no coincide con la velocidad máxima $\mathrm{v}_{\mathrm{m}}$ del viento). El material del halo que se acerca al observador y el material que está por delante del disco estelar formarán la parte del perfil de emisión correspondiente a altas frecuencias (lado izquierdo). En cambio la parte correspondiente a las menores frecuencias del perfil de emisión (lado derecho) es formada sólo por el material del halo que se aleja, ya que la contraparte del material que está por delante de la estrella está justamente bloqueada por la estrella (región 3). Por lo tanto, todas las contribuciones en conjunto forman una emisión asimétrica, ya que la región con velocidades positivas que emite es mayor que la análoga con velocidades negativas.

El motivo por el cual el perfil de emisión tiene un máximo en cero y decae hacia frecuencias desplazadas en $\pm \mathrm{v}_{\mathrm{m}}$, puede ser explicado en términos geométricos. Las áreas con igual velocidad proyectada que emiten son muy diferentes unas o otras. La mayor de ellas corresponde a la que tiene velocidad proyectada igual a cero y, por eso, la emisión es máxima en $\mathrm{v}=0 \mathrm{~km} / \mathrm{s}$. Como no hay iones que absorban o emitan a velocidades mayores que $\pm \mathrm{v}_{\mathrm{m}}$, el perfil queda restringido a este rango de velocidades.

Por lo tanto, si superponemos los perfiles de absorción y emisión, el perfil resultante es un perfil P Cygni (fig. 2.3. derecha).

Por completitud, hay que remarcar que un perfil P Cygni se formará de la manera descripta siempre y cuando en el viento haya cantidades suficientes de iones que absorban y emitan. Contrariamente, si la densidad es baja, el perfil se vuelve más débil, tanto en profundidad como en intensidad. Por ejemplo, cuanto menor sea la velocidad del material absorbente, $\mathrm{v}_{\mathrm{m}}$, más angosto se volverá el perfil. Este y otros efectos pueden modificar la forma del perfil (ver secc. 2.9.3. 


\subsection{Parámetros del viento}

El viento se define como un flujo continuo de material que escapa de la superficie de la estrella e inyecta ese material al medio interestelar circundante. El viento estelar está formado por partículas cargadas, principalmente protones y electrones, aunque también pueden contener átomos ionizados o neutros de otros elementos. Si bien es sabido que todas las estrellas poseen vientos, en las estrellas masivas este proceso es muy notorio ya que las pérdidas de masa son del orden de $10^{-7} \mathrm{M}_{\odot} /$ año a $10^{-4} \mathrm{M}_{\odot} /$ año.

Dadas las características observadas que produce el fenómeno de un viento estelar, es necesario definir cuáles son las cantidades o parámetros más importantes que lo definen y cómo éstos se pueden obtener a partir de las observaciones.

Tasa de pérdida de masa $(\dot{M})$ : es la cantidad de masa que pierde la estrella por unidad de tiempo y se mide en $\mathrm{M}_{\odot} /$ año. Como se mencionó anteriormente, conocer el comportamiento de esta propiedad es fundamental para entender la evolución, ya que la masa es un ingrediente fundamental en los cálculos de los modelos evolutivos. Pero también es importante para entender la evolución química y dinámica de las galaxias huéspedes y la tasa de formación estelar que ella desencadena.

Velocidad terminal $\left(\mathbf{v}_{\infty}\right)$ : es la máxima velocidad que alcanzan las partículas que fueron expulsadas de la estrella cuando éstas están muy alejadas de la superficie de la misma y su unidad es km/s. Medir esta velocidad también es importante porque de ella depende la energía que se está inyectando al medio circundante y al medio interestelar.

Para poder determinar los valores de estos parámetros, es necesario contar con líneas espectrales que estén afectadas por el viento o imágenes donde se vea claramente cómo es la iteracción del viento con el medio circundante. Para obtener la $\mathrm{v}_{\infty}$ por lo general se usan observaciones espectroscópicas ultravioletas de líneas que despliegan el típico perfil $\mathrm{P}$ Cygni. De ellas se usa el ala azul de la componente en absorción saturada, determinando fácilmente por efecto Doppler la velocidad terminal. Estas líneas UV aparecen en las estrellas muy calientes, es decir en tipos espectrales OB más tempranos que B0-B1.5, pero para vientos más fríos y densos, la línea $\mathrm{H}_{\alpha}$ nos brinda información acerca de este parámetro (Puls et al. 2008). Esta línea es fundamental ya que:

- Al ser una línea de recombinación, su ancho equivalente incrementa con el cuadrado de la densidad del viento, más precisamente con $\dot{M}^{2} /\left(R_{\star}^{2} v_{\infty}^{3}\right)$. Por este motivo son mejores indicadores de la tasa de pérdida de masa que las líneas de resonancia (cuyas opacidades son $\propto \rho$ ).

- Es una de las líneas más intensas que se presentan en el óptico.

- Su comportamiento está muy bien estudiado. 
Es necesario remarcar que para vientos ópticamente delgados, o poco densos, por lo general la línea $\mathrm{H}_{\alpha}$ se encuentra en absorción pura, lo cual hace imposible determinar $\mathrm{v}_{\infty}$ (esto es conocido como problema de vientos débiles, Puls et al. 2008).

A modo de comentario final, es necesario tener en cuenta que a la hora de modelar los vientos de las estrellas masivas, se va variando los parámetros del viento $\left(\dot{\mathrm{M}}\right.$ y $\left.\mathrm{v}_{\infty}\right)$ hasta conseguir un buen ajuste. Pero para llegar a este punto de análisis, los parámetros estelares (ver secc. 2.4 deben estar previamente bien determinados, ya que las condiciones fotosféricas también influyen en los procesos radiativos que se pretenden modelar. Una vez determinados los parámetros del viento a partir de las observaciones, no se tiene que perder de vista que estos parámetros van a depender fuertemente de cuán sofisticados sean los códigos de cálculo, ya que éstos se obtienen mediante modelos enmarcados por determinadas hipótesis.

\subsection{Medición de los parámetros estelares}

En esta sección comentaremos cuáles son los principales métodos que nos permitirán estimar, a partir de las observaciones, los diferentes parámetros estelares.

\subsubsection{Temperatura efectiva $\left(\mathrm{T}_{\text {ef }}\right)$}

La $\mathrm{T}_{\mathrm{ef}}$ es uno de los parámetros estelares fundamentales de las estrellas y existen varios métodos para poder calcularla. El método que utilizaremos en este trabajo es mediante la síntesis espectral, que consiste en modelar o ajustar ciertas líneas espectrales dignóstico empleando perfiles de línea sintéticos. Pero también es posible determinar la $T_{\text {ef }}$ a partir del ajuste de la distribución espectral de energía con modelos de atmósferas estelares como así también, a partir de los índices de color fotométricos de los sistemas de Ginebra Cramer 1999 y Strömgren Strömgren 1966 empleando tablas de calibración, o mediante mediciones de anchos equivalentes de ciertas líneas espectrales (Lennon et al. 1992, 1993a. McErlean et al. 1999a).

Para las estrellas de tipo espectral B, el principal indicador de temperatura efectiva en el rango óptico del espectro son las líneas de Si (Becker \& Butler 1990 Kilian et al. 1991. McErlean et al. 1999b, que muestran una intensidad relativa alta en tres estados distintos de ionización: Si III/Si IV para estrellas con tipos espectrales más tempranos (B0-B1) y Si II/Si III para los más tardíos (B3-B9), con una superposición en las estrellas B1.5-B2. En la fig. 2.4 se muestra el resultado final de un ajuste de líneas espectrales para diferentes tipos de estrellas BSGs, donde también se ve claramente la sensibilidad de las líneas de Si IV y Si III con $\mathrm{T}_{\text {ef }}$ : si la temperatura efectiva de los objetos es mayor, las líneas de Si IV se intensifican mientras que las de Si III disminuyen. También es posible obtener una estimación de la $T_{\text {ef }}$ para las estrellas BSGs de temprano tipo espectral mediante

\footnotetext{
${ }^{6}$ Como por ejemplo los modelos de Kurucz 1979 o de Hubeny \& Lanz 1995a TLUSTY). Estos modelos calculan una atmósfera plano-paralela en equilibrio hidrostático y NLTE, y desprecian el efecto de un viento en las opacidades.
} 
los balances de ionización del He (Markova \& Puls 2008), ya que en estas estrellas se intensifican las líneas de He II (ver fig. 2.4).

\subsubsection{Gravedad superficial $(\log g)$}

La gravedad superficial constituye otro de los parámetros estelares fundamentales, y antes de comentar el método para determinarlo, es necesario introducir un fenómeno conocido como efecto Stark. Este efecto es el mecanismo de ensanchamiento por presión más importante en las líneas de $\mathrm{H}$ de las estrellas calientes, y es causado por colisiones coulombianas. Es así que las alas de las líneas de la serie de Balmer son muy sensibles a los cambios en la densidad electrónica, la cual está directamente asociada con la aceleración gravitatoria. Por este motivo, las líneas que se utilizan para determinar la gravedad superficial son $\mathrm{H}_{\gamma}$ (4341 $\AA$ ) y $\mathrm{H}_{\delta}(4102 \AA)$, ya que $\mathrm{H}_{\alpha}(6563 \AA)$ y $\mathrm{H}_{\beta}(4861 \AA)$ suelen estar afectadas por la emisión del viento. El método consiste en el ajuste de perfiles sintéticos a los observados de $\mathrm{H}_{\gamma} \mathrm{y} \mathrm{H}_{\delta}$, similar al mostrado en la fig. 2.4 para calcular la $\mathrm{T}_{\mathrm{ef}}$. Sin embargo, hay que tener en cuenta, que lo que uno mide, usando el método de ajuste de perfiles de líneas, es una gravedad superficial efectiva $\left(\mathrm{g}_{\mathrm{ef}}\right)$ que está afectada por el movimiento de rotación de la estrella. Por lo tanto, si se busca determinar la masa espectroscópica del objeto, esta gravedad debe ser corregida por la fuerza centrífuga. De esta manera, el factor de Eddington también se ve afectado (ver ec. 2.11.

\subsubsection{Radio $\left(\mathrm{R}_{\star}\right)$}

El radio estelar es el último de los parámetros estelares fundamentales cuyos métodos para determinar su valor pasaremos a describir. Es, quizás, el parámetro más complicado de determinar ya que no es un parámetro libre al momento de realizar ajustes de perfiles de líneas, sino que debe obtenerse mediante algún otro método, que, en muchos casos, son indirectos.

El radio estelar puede determinarse a partir de la magnitud bolométrica $\left(\mathrm{M}_{\text {bol }}\right)$, la cual está relacionada con la luminosidad (L) de la estrella mediante la fórmula de Pogson, y también está relacionada con la magnitud absoluta $\left(\mathrm{M}_{\mathrm{v}}\right)$ a través de la corrección bolométrica $(\mathrm{CB})$ :

$$
\begin{gathered}
\mathrm{M}_{\text {bol }}=4,8-2,5 \log \left(\mathrm{L} / \mathrm{L}_{\odot}\right), \mathrm{L}=4 \pi \sigma \mathrm{R}_{\star}^{2} \mathrm{~T}_{\mathrm{ef}}^{4}, \\
\mathrm{M}_{\text {bol }}=\mathrm{M}_{\mathrm{v}}+\mathrm{CB} .
\end{gathered}
$$

donde $\sigma\left(\simeq 5.67 \times 10^{-5} \mathrm{erg} \mathrm{cm}^{-2} \mathrm{~s}^{-1} \mathrm{~K}^{-4}\right)$ es la constante de Stefan-Boltzmann. Por otro lado la magnitud visual absoluta está relacionada a su vez con la distancia (d) según la siguiente expresión:

$$
\mathrm{Mv}=\mathrm{m}_{\mathrm{v}}+5-5 \log (\mathrm{d})-\mathrm{A}_{\mathrm{v}}
$$




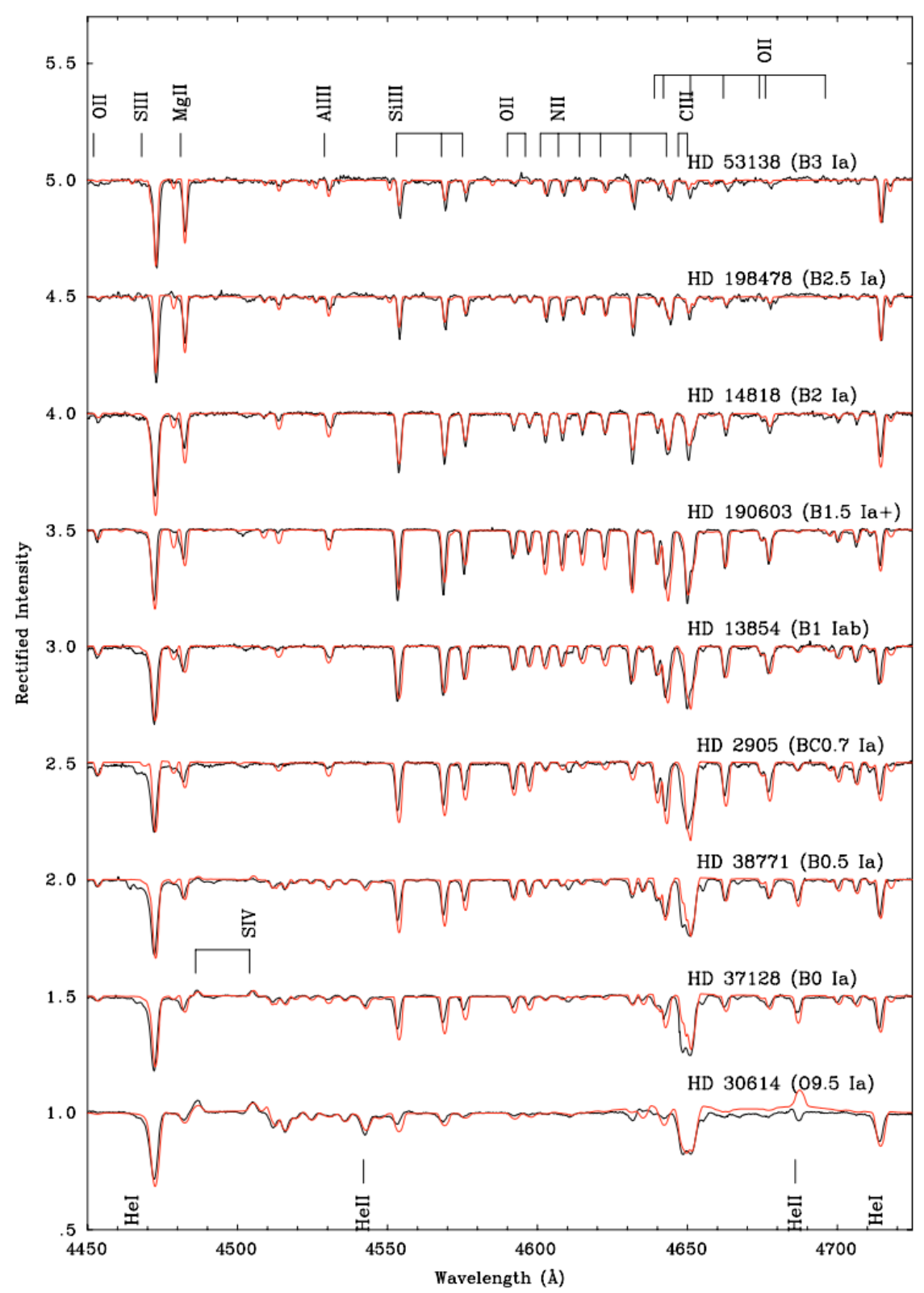

Figura 2.4. Espectros observados (en negro) de estrellas supergigantes B de diferentes tipos espectrales. En rojo se muestra el espectro sintético. Extraído de Crowther et al. 2006a). 
donde $\mathrm{m}_{\mathrm{v}}$ es la magnitud aparente y Av es el coeficiente de absorción interestelar. Por lo tanto, si conocemos la distancia podemos determinar la luminosidad para luego obtener el radio (sabiendo de antemano la temperatura efectiva). A continuación presentamos otros métodos más comúnmente usados para conocer la distancia o el radio:

- Método de las paralajes trigonométricas $\left(\pi["]=\mathbf{d}^{-1}\left[\mathbf{p c}^{-1}\right]\right)$. La mayoría de los objetos cercanos cuentan con mediciones de paralajes trigonométricas, con lo cual la determinación de la distancia es directa. El relevamiento de mediciones de paralaje más importante y conocido es el del satélite HIPPARCOS y próximamente Gaia.

- Diámetro angular $\left(\theta["]=206265 \mathbf{R}_{\star} / \mathbf{d}\right)$. El diámetro angular se obtiene a partir de observaciones interferométricas (ver Hanbury Brown et al. 1974 , Pasinetti Fracassini et al. 2001, por lo que el conocer la distancia nos permitiría determinar el radio.

- Distribución espectral de energía (SED 7 : El ajuste de la SED mediante modelos de atmósferas es un método directo para calcular el radio, la temperatura efectiva y el exceso de color. En esta tesis se utilizó la interface interactiva BeSOS8 8 para ajustar el mejor modelo atmosférico (TLUSTY o Kurucz Hubeny \& Lanz 1995a: Kurucz 1979. BeSOS accede a los datos fotométricos de cualquier estrella disponibles en los catálogos que se encuentran en VizieR y la distancia (paralaje) es obtenida del catálogo de HIPPARCOS. De esta manera BeSOS provee, para un dado exceso de color, un nuevo conjunto de parámetros estelares $\left(\mathrm{T}_{\mathrm{ef}}, \log \mathrm{g}\right.$ y $\left.\mathrm{R}_{\star}\right)$.

- Flujo teórico: el flujo teórico obtenido a partir del ajuste espectral de líneas permite obtener una estimación del radio mediante iteraciones que requieren un valor inicial de $R_{\star}$ que, por lo general, se obtiene a partir de caminos evolutivos (ver detalles en Kudritzki (1980).

- Método del salto de Balmer: empleando el método espectrofotométrico BCD Barbier \& Chalonge 1941, Chalonge \& Divan 1973 se pueden medir de forma directa la intensidad (D) y posición $\left(\lambda_{1}\right)$ del salto de Balmer. Estos dos valores permiten obtener determinaciones muy precisas de la magnitud bolométrica y la $\mathrm{T}_{\text {ef }}$ empleando las tablas de calibración BCD Zorec \& Briot 1991 Zorec et al.2009a. Luego, a partir de estas cantidades se puede calcular el radio estelar.

El radio no es un parámetro fácil de determinar, ya que usando los diferentes métodos mencionados a veces se llega a diferentes resultados, y las discrepancias están relacionadas con los errores intrínsecos de cada sistema de medición, a la rotación del objeto o al hecho de que la estrella puede ser parte de un sistema múltiple o poseer enrojecimiento intrínseco producido por la presencia de materia circunestelar que vela las características propias de la superficie de la estrella.

\footnotetext{
${ }^{7}$ Por sus siglas en inglés

${ }^{8}$ The "Be Stars Observation Survey". http://besos.ifa.uv.cl
} 


\subsubsection{Luminosidad y masa}

Una vez que el radio, la temperatura efectiva y la gravedad superficial (corregida por rotación $\sqrt{9}$ son determinadas, se obtiene la masa (usualmente llamada masa espectroscópica) mediante la siguiente expresión:

$$
\mathrm{M}_{\star}=\mathrm{g} \mathrm{R}_{\star}^{2} / \mathrm{G},
$$

y la luminosidad puede determinarse a partir de la ec. 2.1.

\subsubsection{Velocidades de turbulencia y rotación}

Microturbulencia $\left(\mathbf{v}_{\text {mic }}\right)$ :

Esta velocidad, usualmente denotada con la letra griega $\xi$, es una componente no térmica del campo de velocidades de la fotosfera, y tiene una relación de escala más pequeña que el tamaño de la región donde se forman las líneas fotosféricas Cantiello et al. 2009. Estos campos de velocidades son causados por movimientos convectivos de las capas que están por debajo de la superficie de la estrella. Esto genera un ensanchamiento adicional en las líneas fotosféricas, lo que implica que al realizar ajustes de perfiles es necesario agregar una componente de velocidad, adicional a la velocidad térmica, de unos pocos $\mathrm{km} / \mathrm{s}$ para conseguir un buen ajuste Aerts et al.2010a. Es discutible la presencia de este campo de velocidades ya que no es claro si realmente existe un mecanismo que lo produce o si surge a partir de las limitaciones propias de los modelos de atmósferas (una discusión detallada puede encontrarse en el trabajo de Markova \& Puls 2008.

Macroturbulencia $\left(\mathbf{v}_{\text {mac }}\right)$ :

En contraste a la $\mathrm{v}_{\text {mic }}$, la velocidad de macroturbulencia se refiere a campos de velocidades con escalas mayores al camino libre medio de los fotones Aerts et al. 2009b), lo que se traduce en agregar a los perfiles sintéticos un ensanchamiento de algunos $\mathrm{km} / \mathrm{s}$, para lograr un buen ajuste. Como puede verse en la fig. 2.5. los valores que puede tomar esta $\mathrm{v}_{\text {mac }}$ pueden ser muy altos e incluso superar ampliamente la velocidad del sonido en estrellas masivas Howarth et al. 1997a Lefever et al. 2007 Markova \& Puls 2008. En la fig. 2.5 también puede apreciarse empíricamente que los valores observados de $\mathrm{v}_{\text {mac }}$ parecieran decrecer con los tipos espectrales más tardíos. Estos valores de $v_{\text {mac }}$ también dependen de la definición del perfil teórico que se use para ajustarla (perfiles gaussianos o perfiles radialestangenciales), lo cual genera una gran dispersión, principalmente en los tipos espectrales B1 y B2.

Estos movimientos turbulentos afectan tanto a las líneas fotosféricas como a las líneas formadas en el viento, adquiriendo valores mayores de velocidad en estas últimas. Es por este motivo que, por lo general, cuando se determinan los valores de $\mathrm{v}_{\text {mac }}$ mediante un proceso de ajuste de perfiles teóricos a los perfiles de líneas observados, se utilizan las líneas fotosféricas, ya que si la línea está afectada por el viento, se obtienen valores sobreestimados.

Si bien las causas de este fenómeno no son claras, Aerts et al. 2009a reportaron una relación teórica entre el ensanchamiento producido por la velocidad de macroturbulencia

$$
{ }^{9} \mathrm{~g}_{\text {corr }}=\mathrm{g}_{\text {ef }}+(\mathrm{v} \text { sen } \mathrm{i})^{2} / \mathrm{R}
$$




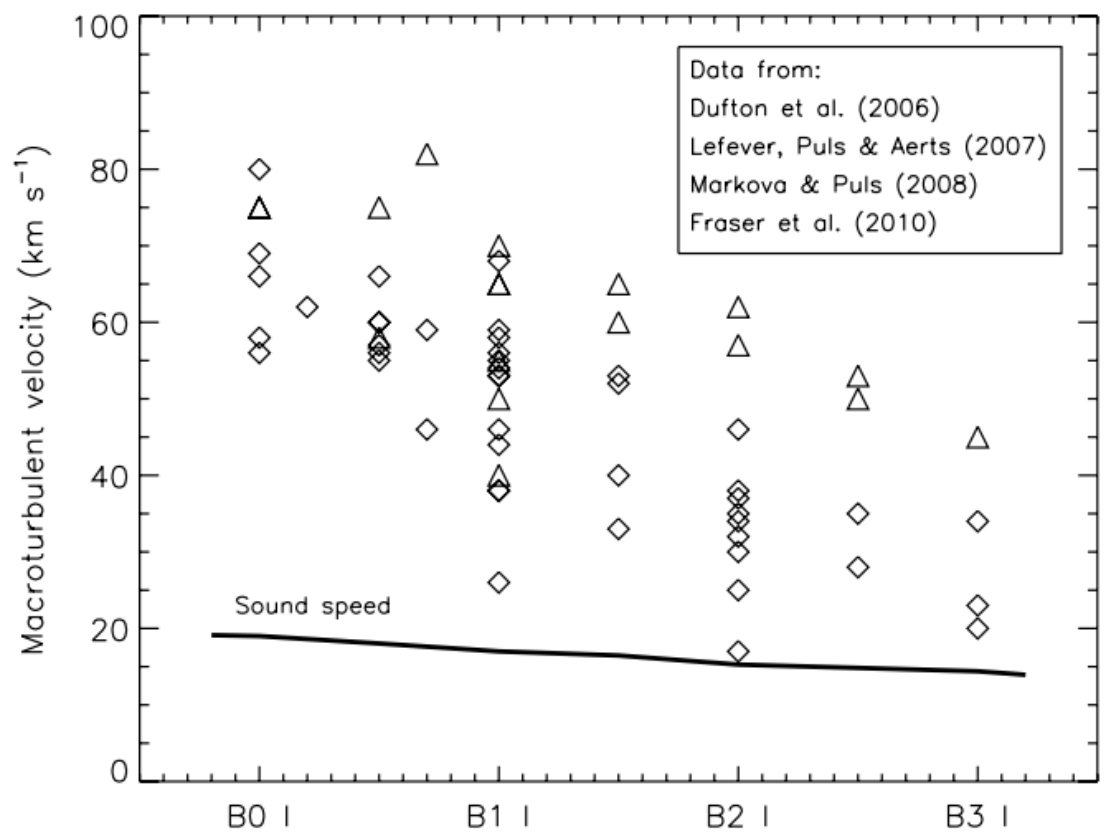

Figura 2.5. Valores determinados para la velocidad de macroturbulencia en función del tipo espectral para diferentes estrellas B supergigantes. Los diferentes símbolos se refieren al tipo de perfil que se utilizó para determinar la $\mathrm{v}_{\text {mac }}$ : diamantes para perfiles gaussianos y triángulos para perfiles radiales-tangenciales. La línea negra continua representa la velocidad del sonido en la fotosfera de las estrellas. Extraído de Simón-Díaz et al. 2010. 

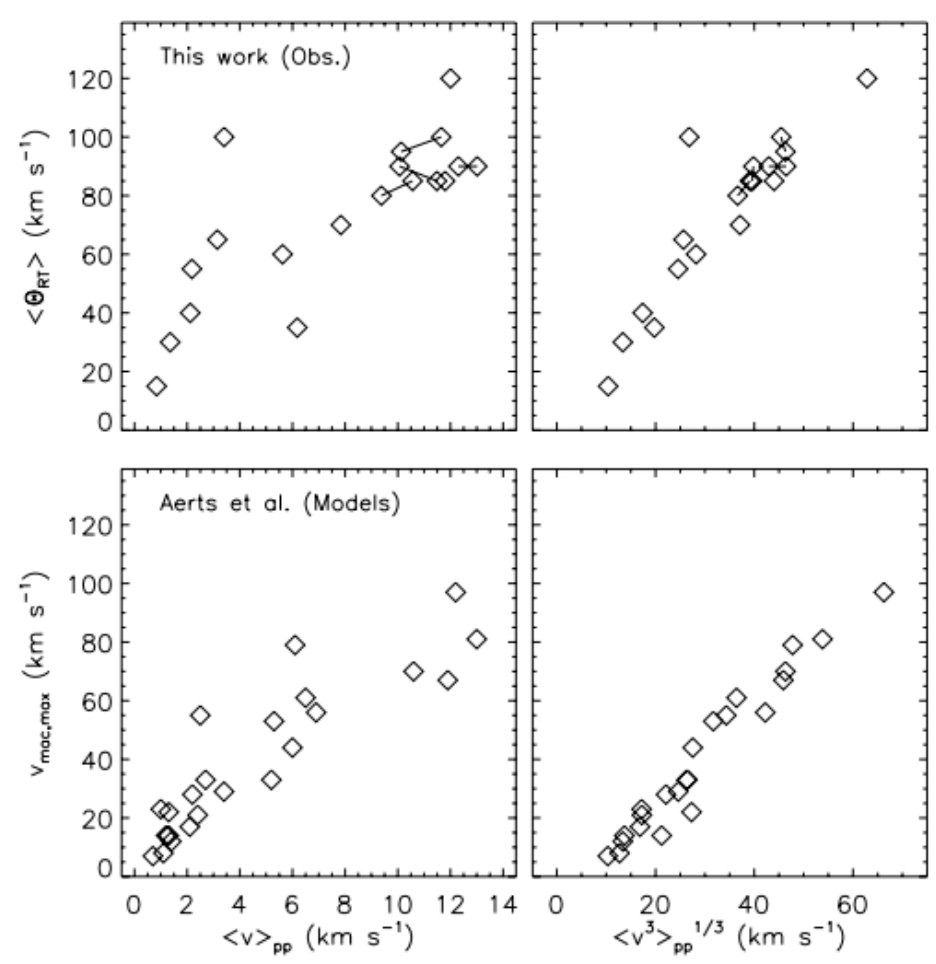

Figura 2.6. Paneles superiores: relación empírica encontrada por Simón-Díaz et al. 2010, "this work" en la fig.) entre los valores medios determinados para la velocidad de macroturbulencia $\left(<\Theta_{\mathrm{RT}}>\right)$ y las amplitudes pico a pico del análisis del primer y tercer momento de las líneas. En los paneles inferiores se muestran las predicciones de una relación similar, determinada por Aerts et al. 2009a. Extraído de Simón-Díaz et al. 2010.

y las variaciones de los perfiles de líneas. Estos autores atribuyen esta relación a un efecto colectivo de pulsaciones. En el mismo sentido, Simón-Díaz et al. (2010) encontraron una correlación observacional similar a la encontrada por Aerts et al. 2009a), como puede apreciarse en la fig. 2.9 0 Otras posibles causas podrían estar asociadas a ondas accionadas estocásticamente por fluctuaciones turbulentas de presión en capas intermedias entre el núcleo y la fotosfera, o en las capas por debajo de las zonas de convección (ver Godart et al.2017, y sus referencias).

Las variaciones fotosféricas detectadas en las observaciones espectroscópicas de las estrellas BSGs (ver secc. 2.9.2 conducen a que los valores para $\mathrm{v}_{\mathrm{mac}}$ no sean iguales para diferentes épocas Lefever et al. 2007 Markova \& Puls 2008.

\section{Velocidad de rotación proyectada (v sen i):}

La velocidad de rotación es otro de los parámetros fundamentales de las estrellas y es, al igual que la pérdida de masa, un factor crucial que influye directamente en la evolución

\footnotetext{
${ }^{10} \mathrm{La}$ variación de los perfiles de línea está en función de los valores de la amplitud pico a pico del primer momento (velocidad radial) y tercer momento (asimetría) de las líneas. Una descripción del análisis de momentos puede verse en el apéndice 6
} 


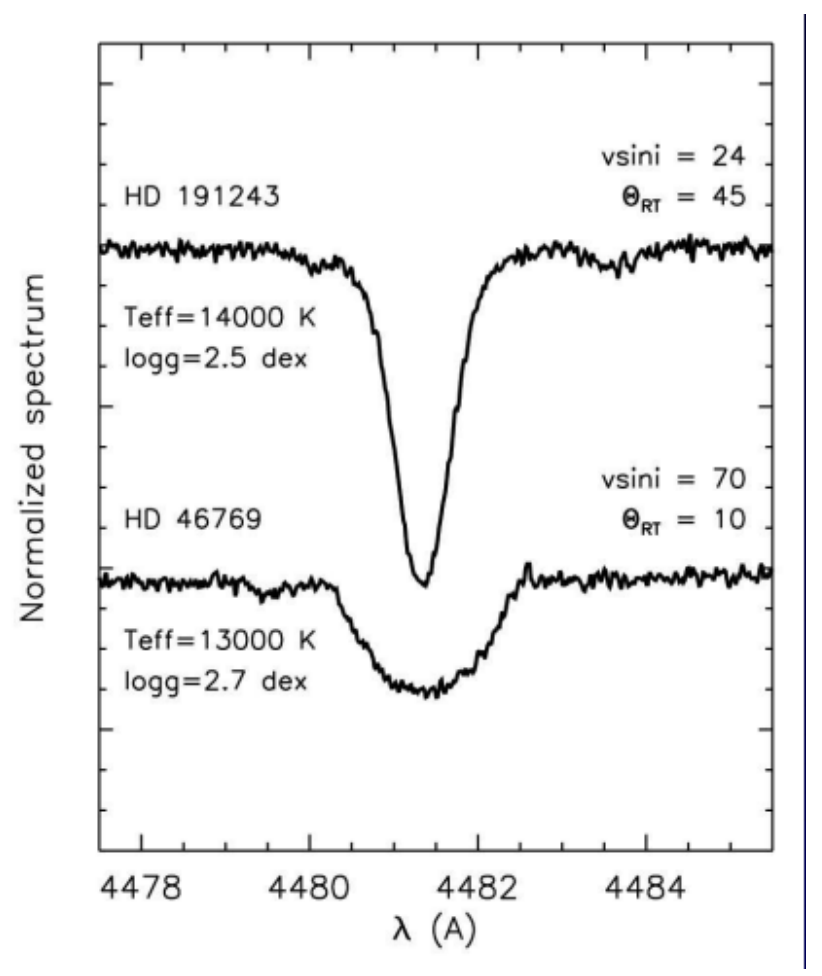

Figura 2.7. Arriba: observación de un perfil de línea dominado por macroturbulencia. Abajo: perfil de línea observado dominado por rotación. Extraído de Simon-Diaz et al. 2013.

estelar Meynet \& Maeder 2000 Saio et al.2013a). Por eso es importante poder discriminar entre esta velocidad de rotación proyectada y la velocidad de macroturbulencia.

Si bien en algunos casos es posible distinguir si en el perfil de línea predomina la rotación por sobre otros efectos de ensanchamiento, por lo general separar adecuadamente las diferentes componentes no es sencillo. Por ejemplo, tanto la macroturbulencia como la rotación mantienen el ancho equivalente y producen cambios "sólo" en la forma de la línea Markova \& Puls 2008, como se muestra en la fig. 2.7

Ante esta dificultad existen varios caminos para poder medir vsen i con precisión:

- El método más sencillo es acudir, en primera instancia, a la calibración v sen i vs tipo espectral para estrellas BSGs galácticas realizada por Abt et al. 2002). Adicionalmente a esta velocidad se le debe agregar el ensanchamiendo debido a los efectos de macroturbulencia para lograr un buen ajuste Markova \& Puls 2008.

- A partir de la medición del ancho equivalente de las líneas a tratar, Ryans et al. 2002 desarrollaron perfiles sintéticos cuya convolución es comparada con la observación tanto para estimar vsen i como $\mathrm{v}_{\mathrm{mac}}$. Finalmente, la suma de los cuadrados de las diferencias provee la calidad del ajuste en cada punto del plano ( $\mathrm{v}_{\mathrm{mac}}$, v sen i). Este método arroja resultados confiables si las observaciones tienen una relación señal ruido $\mathrm{S} / \mathrm{N}>500$. 
- Simón-Díaz \& Herrero 2007a implementaron las técnicas del análisis de la Transformada de Fourier propuestas previamente por Gray 1976). Los perfiles pueden estar ensanchados no sólo por los efectos que se discuten previamente, sino que también puede haber efectos instrumentales. Pero este método se basa en que sólo la función de rotación tiene ceros en su transformada de Fourier, y sólo el primero de estos ceros está relacionado con la velocidad de rotación. Cabe mencionar que este método requiere espectros de muy alta resolución espectral $(\mathrm{R} \sim 40000)$.

\subsubsection{Composición química}

La determinación de las abundancias químicas constituye otro elemento de juicio como diagnóstico y verificación de la teoría de evolución estelar y modelado de las líneas espectrales. Para determinar abundancias de un elemento químico existen al menos dos maneras. Una de ellas es mediante la curva de crecimiento, que consiste en una relación matemática entre la abundancia química y el ancho equivalente (EW, por sus sigla en inglés.) de la líned ${ }^{11}$ del átomo/ion en cuestión. El EW se expresa independientemente de la longitud de onda como $\log (E W / \lambda)$ en la ordenada al origen. Como este ancho depende del número de partículas que forman la línea, en el eje de las absisas de la curva de crecimiento va representado el $\log \left(\mathrm{Ag}_{\mathrm{f}}\right)$, donde $\mathrm{A}$ es la abundancia del elemento respecto a la del $\mathrm{H}\left(\mathrm{N}_{\mathrm{E}} / \mathrm{N}_{\mathrm{H}}\right)$ y $\mathrm{g}_{\mathrm{f}}$ es la fuerza del oscilador de la líned Más detalles sobre este método pueden verse por ejemplo en Lamers 1972 donde se analizan las abundancias de diferentes iones presentes en la estrella BSG $\epsilon$ Ori (B0Ia).

Por otro lado, la síntesis espectral es el método que generalmente se usa hoy en día. Este análisis se basa en un modelo de atmósfera estelar detallado que predice las intensidades de las líneas y los perfiles de las mismas. Estos se comparan luego con las observaciones, y los parámetros inicialmente adoptados se alteran hasta que se logra un buen ajuste. El resultado de un procedimiento como éste se mostró anteriormente en la fig. 2.4.

La abundancia química superficial es un importante indicador del estado evolutivo y existen considerables evidencias de peculiaridades químicas en la fotosfera de las estrellas masivas McErlean et al. 1999b; Searle et al. 2008a. Walborn 1976 fue uno de los primeros en observar anomalías de carbono y nitrógeno en las estrellas BSGs y lo condujo a elaborar el sistema de clasificación espectral BN y BC, donde BN y BC presentan anomalías en las líneas de $\mathrm{N}$ y $\mathrm{C}$, respectivamente. Las observaciones no sólo revelan que existen anomalías químicas, sino que también evidencian la variabilidad en las abundancias de $\mathrm{C}$ y N McErlean et al. 1999b. Crowther et al. 2006a y Searle et al. 2008a también midieron abundancias de CNO en muestras de más de 20 estrellas BSGs y reportaron evidencia de material parcialmente procesado en la superficie de la mayoría de los objetos. Los estudios que realizaron en ambos trabajos, a partir de la síntesis espectral, reveló apartamientos de los cocientes de abundacias de $[\mathrm{C} / \mathrm{N}]$ y $[\mathrm{N} / \mathrm{O}]$ respecto a la abundancia solar. En particu-

\footnotetext{
${ }^{11}$ Se define como el ancho de un rectángulo cuya área es igual al área encerrada por el perfil de la línea. Se mide usualmente en $\AA$.

${ }^{12}$ Es un número adimensional que indica la probabilidad de que se produzca una absorción o una emisión. $\mathrm{Su}$ valor es $\sim 1$ para líneas intensas y $<<1$ para líneas débiles Mihalas 1978.
} 
lar Crowther et al. 2006a realizaron un promedio y calcularon apartamientos de +1.07 dex y +0.74 dex para $[\mathrm{C} / \mathrm{N}]$ y $[\mathrm{N} / \mathrm{O}]$, respectivamente. Cabe mencionar que estos autores comparan dichos resultados con la abundancia solar de $[\mathrm{C}, \mathrm{N}, \mathrm{O}]=[8.39 \pm 0.05,7.78 \pm$ 0.06, $8.66 \pm 0.05$ ] determinada por Asplund et al. 2005).

El enriquecimiento por CNO procesado debería ser más importante en las estrellas más masivas y más luminosas (Lennon et al.|1993a. Es también esperable que las supergigantes en la fase post-RSG tengan más intensificado el CNO procesado Schaller et al. 1992, por lo que una posible manera de distinguir entre los dos grupos de BSGs en un diagrama HR es mediante la determinación de las abundancias de CNO superficiales. Saio et al. 2013b sugieren que las composiciones de CNO se modifican sustancialmente respecto de los valores normales debido a la presencia de regiones convectivas de dragado, la mezcla por rotación y la pérdida de masa.

La composición química en las estrellas masivas es importante al momento de analizar la propia evolución estelar, la dinámica del viento y la composición de las galaxias. Por otro lado, la metalicidad juega también un papel muy importante en la relación momentoluminosidad del viento. Como veremos en las siguientes secciones, esta relación surge de la teoría estándar que explica los vientos de las estrellas masivas y su comportamiento.

\subsection{Teoría de vientos impulsados por radiación}

\subsubsection{Ideas cualitativas}

Los trabajos pioneros en desarrollar las ideas básicas para entender el fenómeno del viento estelar se iniciaron en la década del '70 y fueron realizados por Lucy \& Solomon (1970) y Castor et al. 1975. La idea primordial de esta teoría, la teoría estándar, se basa en que los átomos pueden adquirir impulso mediante la absorción de radiación (idea que ya había sido propuesta por Milne 1924 1926.

Debido a que las estrellas masivas calientes emiten la mayor parte de su radiación en al rango UV, el viento se impulsa, por un lado, debido a la presencia de muchas líneas de absorción en las capas más externas de la atmósfera y, por otro lado, debido al efecto Doppler. Si no fuera porque las capas más externas de la atmósfera se mueven hacia afuera, estas no recibirían radiación de la fotosfera, ya que dicho flujo sería absorbido por los átomos de las capas más profundas, como sucede en un atmósfera estática. Pero la existencia de un gradiente de velocidad en la atmósfera permite que los iones "vean" la radiación de la fotosfera enrojecida y puedan absorberla sin que ésta sea atenuada por las capas más internas. Este proceso hace que la aceleración radiativa debido a las líneas espectrales sea muy efectiva para impulsar el viento de estrellas supergigantes $\mathrm{O}$, B y A, como así también los vientos de las estrellas centrales de una nebulosa planetaria y enanas blancas (Lamers \& Cassinelli 1999a.

Como mencionamos, la capa más profunda de la atmósfera estelar es la fotosfera o superficie estelar, de donde proviene la mayor parte de la radiación de continuo observada. Los fotones provenientes de ella iteractúan con los iones presentes en las regiones cercanas 
y le transfieren su momento. Estos iones pueden ser átomos ionizados de silicio, nitrógeno, carbono, etc. Las interacciones que van a sufrir estos iones con los fotones se pueden describir como sigue:

- Los fotones van a ser absorbidos si sus energías se corresponden a las energías de niveles ligados.

- Supongamos por simplicidad que el ión se mueve en dirección radial con velocidad $\mathrm{v}_{1}$ y que éste absorbe un fotón $\left(\nu_{1}\right)$ que viene en la misma dirección (ver fig. 2.8). Durante el proceso de absorción, el fotón le transfiere momento al ión.

- Luego de un intervalo de tiempo relativamente corto $\left(10^{-8} \mathrm{~s}\right)$ el ión se desexcita, y el electrón realiza una transición al estado fundamental, emitiendo un nuevo fotón $\left(\nu_{2}\right)$ con un ángulo $\alpha$ respecto a la dirección radial.

- Así, el ion es acelerado en una dirección diferente respecto a la inicial con velocidad $\mathrm{v}_{2}$. Suponiendo que el ion puede absorber sólo fotones con frecuencia $\nu_{0}$, y tomando en cuenta que $\mathrm{v} \ll \mathrm{c}$ (con $\mathrm{c} \equiv$ velocidad de la luz) y $h \nu_{0} \ll m c^{2}$ (con $\mathrm{m} \equiv$ masa del ión), se encuentra que el ión adquiere una velocidad cuya componente radial puede expresarse como

$$
v_{2}-v_{1}=\frac{h \nu_{0}}{m c}(1-\cos \alpha)
$$

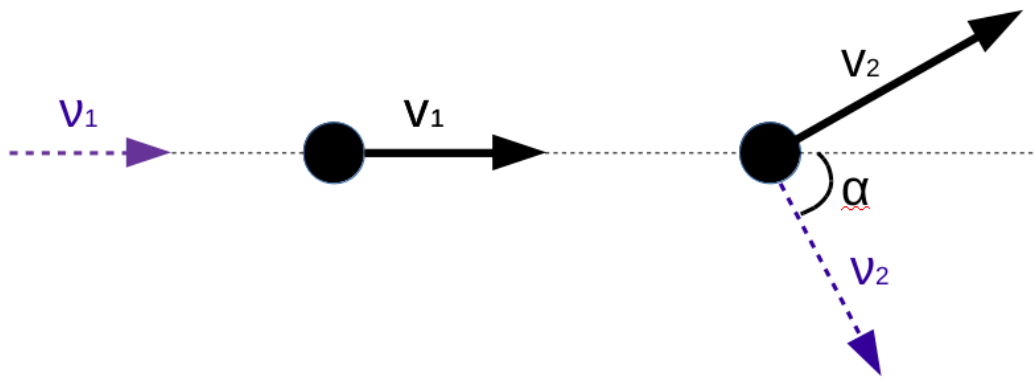

Figura 2.8. Transferencia de momento del fotón al ion y posterior emisión de un fotón con frecuencia $\nu_{2}$ en la dirección $\alpha$.

- El resultado global de la aceleración del ion es la suma de las dos aceleraciones debidas a los procesos de absorción y emisión.

- Como la dirección del fotón emitido es arbitraria (es decir, cualquier dirección tiene la misma probabilidad), el momento medio ganado por el ion después de varios procesos como éste es $h \nu_{0} / c$. 
Finalmente, los iones acelerados transfieren su momento y energía, por medio de colisiones coulombianas al plasma del viento, que básicamente está compuesto por los iones de $\mathrm{H}$ y He, que son los más abundantes, y todo el viento en su totalidad es acelerado en dirección saliente de la estrella. Este análisis queda representado en la fig. 2.9. Cabe mencionar que la transferencia de momento por radiación predice muy bien el comportamiento del viento de las estrellas masivas más calientes $\left(\mathrm{T}_{\mathrm{ef}} \geq 25000 \mathrm{~K}\right)$ ya que sus flujos de radiación son muy intensos. Pero para estrellas más frías, cuyo campo de radiación es débil para iniciar el viento, existen otros mecanismos que son los responsables del proceso de aceleración inicial, como por ejemplo, vientos impulsados por polvo y vientos coronales, entre otros (ver Lamers \& Cassinelli 1999a).

Los primeros estudios teóricos de los vientos impulsados por radiación lograron parametrizar las soluciones del viento con dos parámetros, $\kappa$ y $\alpha$, que miden la cantidad de líneas efectivas intervinientes en el proceso de absorción y la capacidad de los iones para absorber momento, respectivamente. Adicionalmente a estos dos parámetros, Abbott (1982 introduce uno nuevo: el parámetro $\delta$, que considera la estructura de ionización del viento. El motivo de esta incorporación fue para evitar que la ionización sea constante a lo largo del viento pero que no intervenga de manera considerable en la aceleración del mismo. Por este motivo, el valor de $\delta$ para un viento de una estrella OSG es $\sim 0.1$. De esta manera queda enmarcada la teoría CAK (por Castor, Abbott y Klein Castor et al. 1975 para vientos impulsados por radiación. Pauldrach et al. 1986 y Friend \& Abbott (1986 mejoran las soluciones al considerar el tamaño finito del disco estelar, teniendo en cuenta los fotones no radiales, dando lugar a la teoría CAK modificada, o m-CAK como suele denominarse en la literatura.

\subsubsection{Ecuaciones básicas}

Si una estrella pierde masa debido a un viento estelar entonces existe una fuerza dirigida hacia afuera que es mayor que la fuerza gravitatoria. En el caso de las estrellas tempranas esta fuerza es la fuerza de radiación que actúa sobre las líneas y el continuo. La teoría de vientos impulsados por radiación, propone que el viento es estacionario, homogéneo (no existen choques ni aglomeraciones) y esféricamente simétrico. La simplicidad que conllevan estas hipótesis es que todas las fuerzas externas son puramente radiales, con lo cual la rotación, el tamaño finito del disco y los campos magnéticos no son considerados.

En este marco, el flujo total de masa, si la densidad del medio es $\rho$, a través de una superficie esférica de radio $\mathrm{r}$ centrada en la estrella, puede ser representado por la ecuación de continuidad independiente del tiempo, $\frac{1}{r^{2}} \frac{\partial}{\partial r}\left(\rho v r^{2}\right)=0$, adquiriendo la siguiente expresión

$$
\dot{\mathrm{M}}=4 \pi \mathrm{r}^{2} \rho(\mathrm{r}) \mathrm{v}(\mathrm{r})=\text { constante, }
$$

donde $\dot{M}$ es la tasa de pérdida de masa.

La ecuación de movimiento de un viento con estas características es

$$
v \frac{d v}{d r}=-\frac{G M_{\star}}{r^{2}}-\frac{1}{\rho} \frac{d p}{d r}+g_{e}+g_{L},
$$



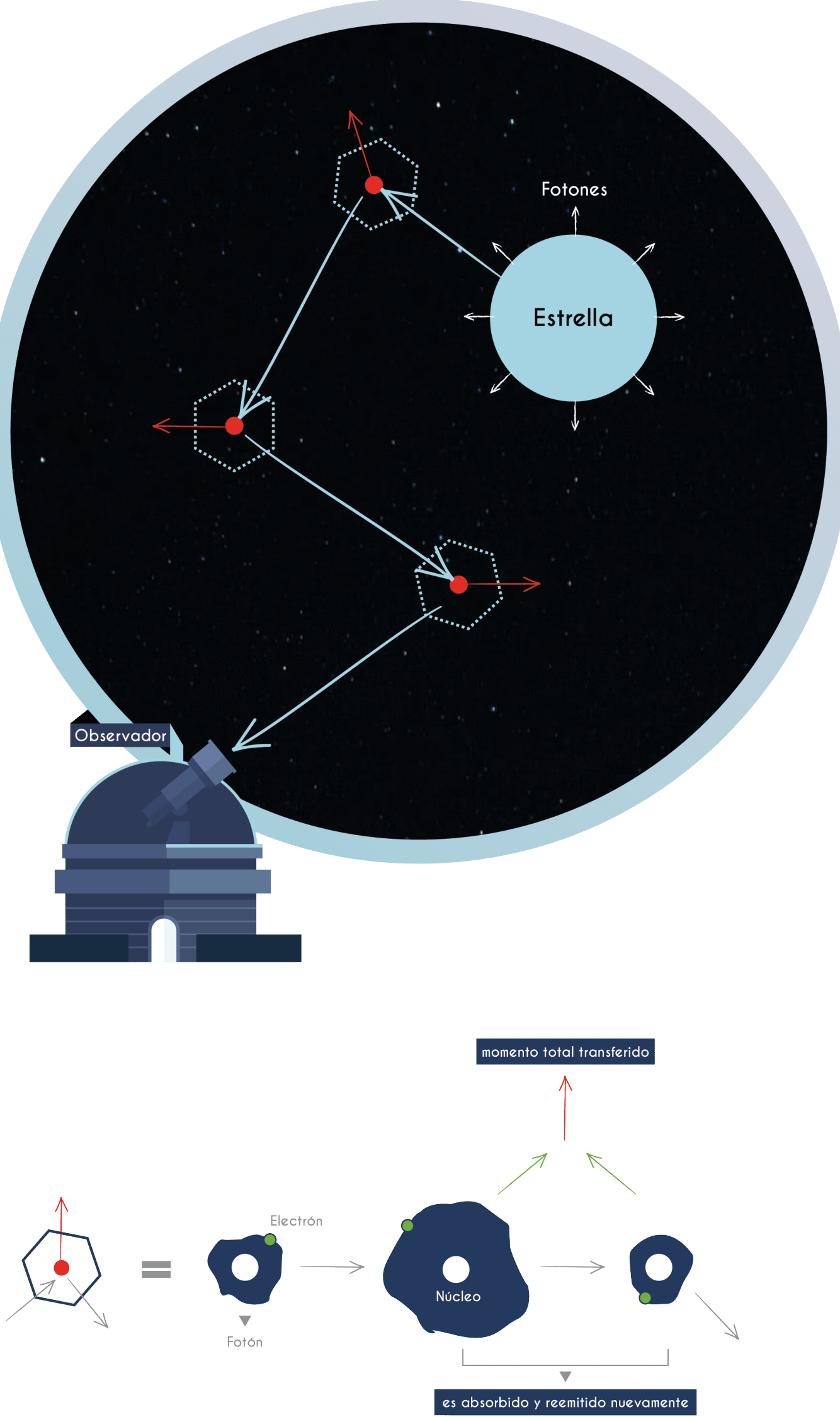

Figura 2.9. Esquema donde se muestra gráficamente el proceso de transferencia de impulso. 
con la aceleración gravitatoria dirigida hacia el centro de la estrella, y el gradiente de la presión del gas y la aceleración radiativa hacia afuera. Esta última está compuesta por dos términos: $g_{e}$ que es la aceleración debida a la opacidad del continuo por la dispersión de electrones libres, entre otro: ${ }^{13}$ y $g_{L}$ que es la aceleración radiativa debida a las líneas, y pueden ser representadas como:

$$
\begin{gathered}
g_{e}(r)=\frac{\sigma_{e} L}{4 \pi r^{2} c} \\
g_{\mathrm{L}}=\frac{\mathrm{F}_{\nu_{0}}}{\mathrm{c}}=\frac{\mathrm{L}}{4 \pi \mathrm{r}^{2} \mathrm{c}} \int_{\text {línea }} \kappa_{\nu} \rho \phi(\nu) \mathrm{d} \nu,
\end{gathered}
$$

donde $\kappa_{\nu}$ es el coeficiente de absorción y $\phi(\nu)$ es la función del perfil de absorción normalizada, es decir,

$$
\int_{-\infty}^{+\infty} \phi(\nu) d \nu=1
$$

\subsubsection{Cálculo de la fuerza de radiación}

Aquí surge el primer desafío, que consiste en modelar y predecir correctamente los dos términos asociados a la fuerza de radiación, la cual depende tanto de la cantidad de flujo radiada por la estrella como de opacidad de las partículas que interceptan esa radiación.

Como el viento de una estrella masiva está completamente ionizado, hay una gran cantidad de electrones libres y son ellos los que le dan significado físico al término $g_{e}$, que es directamente proporcional al coeficiente de absorción para el scattering Thompon $\left(\sigma_{e}\right)^{14}$ multiplicado por el flujo.

De la ec. 2.8 se desprende que la aceleración del continuo tiene la misma dependencia radial que la aceleración gravitatoria $\left(\propto r^{-2}\right)$, con lo que el cociente entre ambas aceleraciones puede ser expresado en términos del cociente entre la luminosidad y la masa de la estrella (L/M), es decir, del factor de Eddington:

$$
\Gamma_{e}=\frac{g_{e}}{g_{\text {Newton }}}=\frac{\sigma_{e} L_{\star}}{4 \pi c G M_{\star}}=7,6610^{-5} \sigma_{e} \frac{L_{\star}}{L_{\odot}}\left(\frac{M_{\star}}{M_{\odot}}\right)^{-1} \Rightarrow \sigma_{e}=\frac{G M_{\star}}{r^{2}} \Gamma_{e}(r)
$$

Supondremos que $\Gamma_{e}$ es una constante, lo cual es cierto si el grado de ionización del viento es constante. Si, además, se supone que el viento es isotérmico y se comporta como un gas perfecto, la presión del gas será $p_{g}=a^{2} \rho$, donde $a$ es la velocidad isotérmica del sonid ${ }^{15}$ Luego, empleando la ecuación de continuidad, la ec. 2.7 resulta

$$
v \frac{d v}{d r}=-\frac{G M_{\star}\left(1-\Gamma_{e}\right)}{r^{2}}+\frac{a^{2}}{v} \frac{d v}{d r}+\frac{2 a^{2}}{r}+g_{L} .
$$

\footnotetext{
${ }^{13}$ Por ejemplo procesos ligado-libre que son válidos para estrellas tempranas.

${ }^{14} \sigma_{e}=\mathrm{s}_{\mathrm{e}} \rho$, con $\mathrm{s}_{\mathrm{e}}$ proporcional a la sección eficaz.

${ }^{15} \mathrm{a}=\sqrt{\mathrm{kT} / \mu}$, donde $\mathrm{k}$ es la constante de Boltzmann, $\mathrm{T}$ es la temperatura y $\mu$ es el peso molecular medio.
} 
Antes de analizar el término $\mathrm{g}_{\mathrm{L}}$ es necesario considerar qué tan opaco es el medio respecto a una determinada frecuencia de radiación $\nu_{0}$. En un medio ópticamente delgado los fotones tendrán muy poca interacción con la materia y sus caminos libres medios serán comparables con las dimensiones del medio, haciendo que éste sea transparente a dichos fotones. En cambio, cuando un medio es ópticamente grueso, la interacción entre la materia y los fotones será mucho mayor, produciendo en estos últimos múltiples desviaciones y logrando que los caminos libres medios sean mucho más pequeños que las dimensiones del medio. Estas dos situaciones suelen representarse por medio de un parámetro que es directamente proporcional al coeficiente de absorción: la profundidad óptica $\tau_{\nu}$. Para un medio ópticamente delgado, $\tau_{\nu} \ll 1$, mientras que para un medio ópticamente grueso $\tau_{\nu}$ $\gg 1$. De esta manera también se definen las líneas ópticamente delgadas $\left(\tau_{\nu} \ll 1\right)$ y ópticamente gruesas $\left(\tau_{\nu} \gg 1\right)$, siendo las primeras muy débiles.

Algo importante a destacar es que la aceleración debido a las líneas ópticamente delgadas, si bien contrarrestan la gravedad hasta cierto punto, no son capaces de impulsar el viento estelar por ellas mismas. Por este motivo, la aceleración del viento sucede gracias a las líneas ópticamente gruesas u opacas. En este caso toda la radiación (alrededor de $\nu_{0}$ ) será absorbida y no dependerá del número de iones absorbentes (como en el caso $\tau_{\nu}$ $\ll 1)$, pero sí de la fracción del flujo estelar alrededor de $\nu=\nu_{0}, \mathrm{~F}_{\nu_{0}}$, que pueda ser absorbido. Esta simplificación se debe a una de las más importantes aproximaciones que tiene la teoría estándar, que es la aproximación de Sobolev (Sobolev 1960). Ésta supone una única región de interacción por efecto Doppler y que el coeficiente de absorción y la densidad no cambian fuertemente a lo largo de dicha región. O, dicho de otra manera, el gradiente de velocidad del fluido es tan grande que las condiciones físicas del medio no cambian drásticamente en dicha región. Esto se cumple si el tamaño geométrico de esta región de interacción es muy pequeña y está dada por:

$$
\Delta \mathrm{r} \simeq \frac{\mathrm{v}_{\mathrm{th}}}{\mathrm{dv} / \mathrm{dr}}
$$

que se conoce como la longitud de Sobolev ( $\left.L_{\text {Sob }}\right)$, la cual es muy pequeña comparada con la altura de escala hidrodinámica, Hp. Esto es:

$$
L_{\mathrm{Sob}} \approx \frac{v_{\mathrm{th}}}{d v / d r}<<H_{p}
$$

con $H_{p}=\frac{\rho}{d \rho / d r}$, donde $\mathrm{v}_{\mathrm{th}}=\sqrt{\mathrm{kT} / \mathrm{m}_{\mathrm{p}}}$ es la velocidad térmica de los protones.

De esta forma el perfil de línea $\phi(\nu)$ puede ser aproximado por una Delta de Dirac y la expresión para la aceleración de una línea ópticamente gruesa queda expresada como:

$$
g_{\text {gruesa }}=\frac{L_{\star}}{4 \pi \rho r^{2} c^{2}} \frac{d v}{d r} .
$$

Combinando las ecuaciones para la aceleración general de una línea (ec. 2.9 y para una línea ópticamente gruesa (ec. 2.15) se obtiene la expresión general para la aceleración de 
la línea que puede ser representada como:

$$
g_{\mathrm{L}}=\frac{L_{\nu_{0}} \nu_{0}}{4 \pi \rho r^{2} c^{2}} \frac{\mathrm{dv}}{\mathrm{dr}}\left(1-\mathrm{e}^{-\tau}\right)
$$

donde $\mathrm{L}_{\nu_{0}}$ es la luminosidad en la frecuencia de la línea y $\tau$ es la profundidad óptica de la línea, que, usando la aproximación de Sobolev, es

$$
\tau \simeq \tau^{\mathrm{Sob}}=\kappa_{\nu} \frac{c}{\nu_{0}} \frac{\mathrm{dr}}{\mathrm{dv}}
$$

De esta manera, la teoría CAK Castor et al. 1975 de los vientos estacionarios impulsados por radiación depende crucialmente del uso de la aproximación de Sobolev para determinar la aceleración o fuerza de la línea.

El siguiente paso consiste en sumar individualmente todas las líneas que contribuyen a la aceleración $\mathrm{g}_{\mathrm{L}}$, es decir, tanto las ópticamente delgadas como las ópticamente gruesas. Esto puede ser convenientemente expresado en términos de la aceleración radiativa debido al scattering de electrones libres multiplicado por un factor $\mathcal{M}(t)$ denominado multiplicador de fuerza.

$$
g_{\mathrm{L}}(r)=g_{\mathrm{e}}^{\mathrm{ref}} \mathcal{M}(t)=\frac{\sigma_{e}^{\mathrm{ref}} L}{4 \pi c r^{2}} \mathcal{M}(t)
$$

donde $\sigma_{e}^{\text {ref }}$ es un valor de referencia para el scattering electrónic ${ }^{16}$ y $\mathcal{M}(t)$ es una función que puede ser parametrizada de la siguiente manera Abbott 1982:

$$
\mathcal{M}(t)=K t^{-\alpha}=k t^{-\alpha}\left(\frac{n_{\mathrm{e}}}{W}\right)^{\delta}
$$

donde $\mathrm{n}_{\mathrm{e}}$ es la densidad electrónica y $\mathrm{W}$ es el factor geométrico de dilución que está dado por

$$
W(r)=\frac{1}{2}\left(1-\sqrt{1-\left(\frac{R_{\star}}{r}\right)^{2}}\right) .
$$

Los parámetros $\mathrm{k}(\mathrm{o} \mathrm{K}), \alpha$ y $\delta$ son los parámetros del multiplicador de fuerza o parámetros de la fuerza de la línea.

Finalmente, la corrección por el tamaño finito del disco estelar (teoría m-CAK Paul-

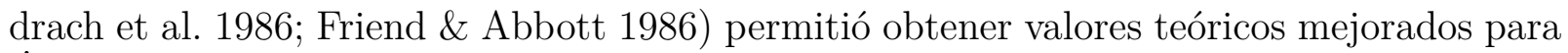
M y para $\mathrm{v}_{\infty}$, ya que, por ejemplo, los valores previos de la tasa de pérdida de masa Castor et al. 1975 eran 2 a 3 veces mayores que los valores observados.

$$
{ }^{16} \sigma_{e}^{\text {ref }}=0.325 \mathrm{~cm}^{2} \mathrm{~g}^{-1} \text { Abbott } 1982 .
$$




\subsubsection{Ecuación de movimiento}

Considerando que la aceleración de radiación en las líneas (ec. 2.16 se puede parametrizar en términos de la luminosidad y el gradiente de velocidad, se obtiene la ecuación de movimiento para un viento conducido por las líneas:

$$
\left(1-\frac{\mathrm{a}^{2}}{\mathrm{v}^{2}}\right) \mathrm{r}^{2} \mathrm{v} \frac{\mathrm{dv}}{\mathrm{dr}}=-\mathrm{GM}\left(1-\Gamma_{\mathrm{e}}\right)+2 \mathrm{a}^{2} \mathrm{r}+\mathrm{C}\left(\mathrm{r}^{2} \mathrm{v} \frac{\mathrm{dv}}{\mathrm{dr}}\right)^{\alpha},
$$

donde la constante $C$ está definida por

$$
C=\frac{\sigma_{\mathrm{e}}^{\mathrm{ref}} \mathrm{Lk}}{4 \pi \mathrm{c}}\left(\frac{\sigma_{\mathrm{e}}^{\mathrm{ref}} \mathrm{v}_{\mathrm{th}} \dot{\mathrm{M}}}{4 \pi}\right)^{-\alpha}\left(\frac{10^{-11} \mathrm{n}_{\mathrm{e}}}{\mathrm{W}}\right)^{\delta},
$$

$\mathrm{y} \mathrm{V}_{\mathrm{th}}$ es la velocidad térmica media de los protones. El factor $\left(\mathrm{n}_{e} / \mathrm{W}\right)$ no es estrictamente constante en el viento, ya que la densidad electrónica $\left(\mathrm{n}_{e} \sim \rho \sim \mathrm{r}^{-2} \mathrm{v}^{-1}\right)$ no presenta la misma dependencia que el factor de dilución $\left(\mathrm{W} \sim \mathrm{r}^{-2}\right)$; sin embargo, si $\delta$ es muy pequeño, dicho cociente se puede suponer constante Lamers \& Cassinelli 1999a.

La ec. 2.21 es una ecuación diferencial no lineal y una estimación de su solución se puede obtener fácilmente si se ignora, en la ecuación de movimiento, la fuerza debida al gradiente de presión del ga: ${ }^{17}$. Esta aproximación es válida en aquellas regiones donde la velocidad del sonido es despreciable comparada con la velocidad del fluido, es decir donde las fuerzas radiativa y de gravedad, dominan sobre el gradiente de presión del gas. Entonces, si se ignora este gradiente, los términos que contienen a la velocidad del sonido, $a$, se anulan en la ec. 2.21, y se tiene,

$$
\mathrm{r}^{2} \mathrm{v} \frac{\mathrm{dv}}{\mathrm{dr}}-\mathrm{C}\left(\mathrm{r}^{2} \mathrm{v} \frac{\mathrm{dv}}{\mathrm{dr}}\right)^{\alpha}=-\mathrm{GM}\left(1-\Gamma_{\mathrm{e}}\right)=\text { constante } .
$$

Esta condición se puede cumplir a cada distancia $r$ sólo si

$$
\mathrm{r}^{2} \mathrm{v} \frac{\mathrm{dv}}{\mathrm{dr}} \equiv \mathrm{D}=\text { constante }
$$

con lo que la ec. 2.23 se reduce a una forma muy simple:

$$
\mathrm{CD}^{\alpha}=\mathrm{D}+\mathrm{GM}_{\star}\left(1-\Gamma_{\mathrm{e}}\right)=\mathrm{D}+\mathrm{GM}_{\star \mathrm{ef}}, \text { donde } \mathrm{M}_{\star \mathrm{ef}}=\mathrm{M}_{\star}\left(1-\Gamma_{\mathrm{e}}\right),
$$

cuya solución se puede aproximar con la siguiente "Ley $\beta$ ":

$$
v(r) \simeq v_{\infty}\left(1-R_{0} / r\right)^{\beta}
$$

donde

$$
R_{0} \simeq R_{\star}\left[1-\left(v_{0} / v_{\infty}\right)^{1 / \beta}\right]
$$

\footnotetext{
${ }^{17}$ Debido a que el término correspondiente a la entalpía en la ecuación de la energía es mucho más chico que el correspondiente a las energías cinética y potencial Vink 2000 Puls et al. 2008.
} 
y $R_{\star}, v_{\infty}$ y $v_{0}$, el radio estelar, la velocidad terminal del viento y la velocidad del viento en la fotosfera $\left(r=R_{\star}\right)$, respectivamente. Cabe mencionar que para esta solución $\beta$ es igual a 0.5 , y que otros valores de $\beta$ corresponden a diferentes generalizaciones.

Kudritzki et al. 1989 mostraron que esta aproximación para la solución de la ecuación de movimiento difiere sólo levemente de la solución exacta. Un ejemplo de esta ley, para un valor de $\beta$ de 0.8 puede verse en la fig. 2.10 .

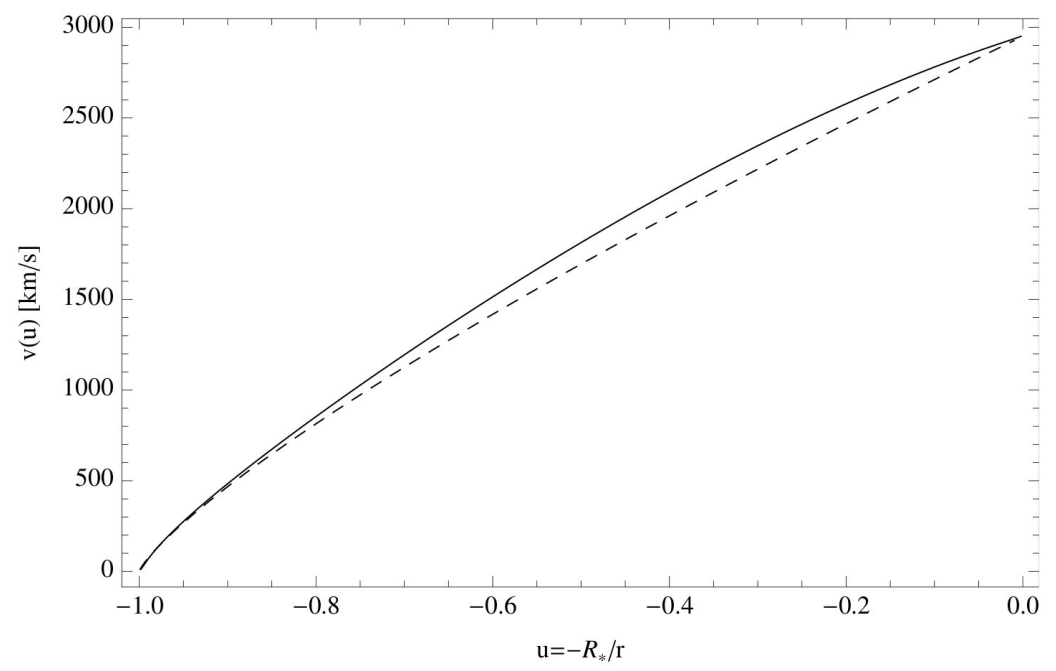

Figura 2.10. Comparación entre el modelo teórico de Kudritzki et al. 1989 (curva continua) y la ley $\beta$ (curva a trazos) para una estrella supergigante $O$ con una $v_{\infty}=2950 \mathrm{~km} / \mathrm{s} \mathrm{y} \beta=0.8$. Extraído de Martins et al. 2005.

Definiendo a la velocidad de escape efectiva como

$$
\mathrm{V}_{\mathrm{esc}}=\sqrt{\frac{2 \mathrm{GM}_{\mathrm{ef}}}{\mathrm{R}_{\star}}}
$$

la teoría de vientos impulsados por radiación Castor et al. 1975, Pauldrach et al. 1986. Kudritzki et al. 1989 predice las siguientes relaciones:

$$
\begin{aligned}
& \left(\frac{\mathrm{V}_{\infty}}{\mathrm{V}_{\mathrm{esc}}}\right)=\sqrt{\frac{\alpha}{1-\alpha}}, \mathrm{y} \\
& \dot{\mathrm{M}} \propto k L_{\star}^{1 / \alpha_{\mathrm{ef}}} M_{\mathrm{ef}}^{1-\frac{1}{\alpha_{\mathrm{ef}}}},
\end{aligned}
$$

con $\alpha_{\mathrm{ef}}=\alpha-\delta \simeq 0.6$ para estrellas $\mathrm{O}$ (Lamers \& Cassinelli 1999b).

Por lo que la relación entre el momento total del viento, $\mathrm{M}_{\infty}$, y la luminosidad de la estrella, L, quedan vinculadas por la siguiente relación:

$$
\dot{M} v_{\infty} \propto R_{\star}^{(-0,5)} L^{\left(1 / \alpha_{\mathrm{ef}}\right)},
$$

que conduce a la relación momento-luminosidad del viento. 
Cabe mencionar que la solución de la teoría estándar es válida si los parámetros k y $\alpha$ son constantes a lo largo del viento y se cumplen otras hipótesis que no fueron mencionadas anteriormente. A saber:

- la aproximación de una estructura núcleo-halo, que no tiene en cuenta la formación continua del viento, es decir, que la fotosfera y el viento no están unificados,

- la ausencia de efectos de scattering múltiple en las líneas. En la teoría CAK cada línea interactúa una sola vez con la radiación continua no atenuada,

- y la carencia de efectos del viento sobre el campo de radiación emergente.

\subsubsection{Relación momento-luminosidad del viento}

Las propiedades de los vientos de las estrellas masivas son muy diversas, y esta diversidad está relacionada con la variación del número efectivo de líneas que impulsan al viento en función del tipo espectral y la metalicidad, y la eficiencia de los efectos de multi-líneas ${ }^{18}$ como función de la densidad del viento (Puls et al. 2008).

Como fue demostrado por Kudritzki et al. 1989 y Puls et al. 1996, una manera muy simple de entender y discutir la fuerza del viento de las estrellas masivas es a través de la relación momento-luminosidad. Ésta es una de las predicciones más importantes de la teoría de vientos impulsados por radiación ya que surge directamente a partir de los parámetros estimados, tanto del viento como de la fotosfera. Kudritzki et al. 1995 definen la tasa del momento del viento $\mathrm{D}_{\text {mom }}$ como:

$$
D_{\mathrm{mom}}=\dot{\mathrm{M}}_{\infty}\left(\mathrm{R}_{\star} / \mathrm{R}_{\odot}\right)^{1 / 2} \propto \mathrm{L}^{1 / \alpha_{\mathrm{ef}}} .
$$

Aplicando logaritmo a ambos lados obtenemos la relación momento-luminosidad modifica$d a$ :

$$
\log D_{\text {mom }}=\mathrm{X} \log \left(\mathrm{L} / \mathrm{L}_{\odot}\right)+\log \mathrm{D}_{0}
$$

que es uno de los mayores logros de la teoría estándar. La fig. 2.11 representa de manera gráfica esta relación para algunas estrellas de la Vía Láctea.

La importancia de esta relación reside en que puede ser utilizada para calcular distancias extragalácticas mediante datos espectroscópicos Kudritzki et al. 1999) y determinar la dependencia de la tasa de pérdida de masa con la metalicidad. En la fig. 2.12 se muestra una relación entre estos dos parámetros, que indica que cuanto menor es la metalicidad menor es el momento del viento.

\footnotetext{
${ }^{18}$ Estos efectos surgen de pensar en la posibilidad de que los fotones interactúen más de una vez en diferentes transiciones cuando se propagan a través del viento y son esenciales para las estrellas calientes ya que tanto la densidad del viento como la intensidad de las líneas en el UV son grandes Puls 1987.
} 


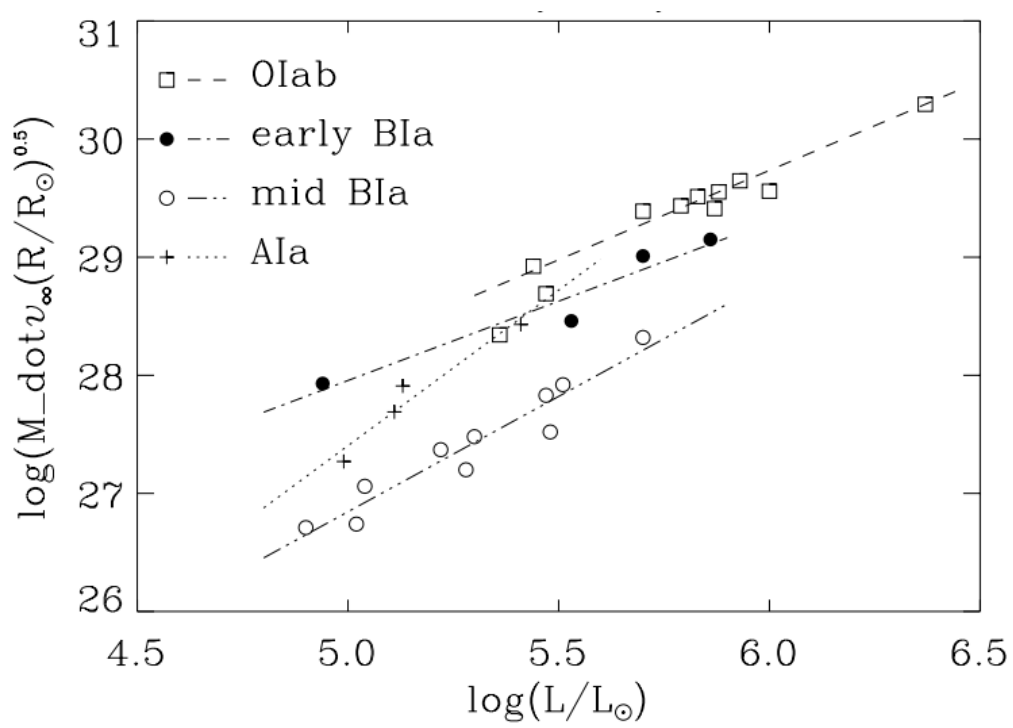

Figura 2.11. Relación momento-luminosidad modificada para estrellas supergiantes O, B (de tipos espectrales temprano y medio) y A de la Vía Láctea determinada empíricamente. Las diferentes rectas corresponden a diferentes ajustes lineales realizados sobre los datos de cada subgrupo. Extraído de Kudritzki et al. 1999.

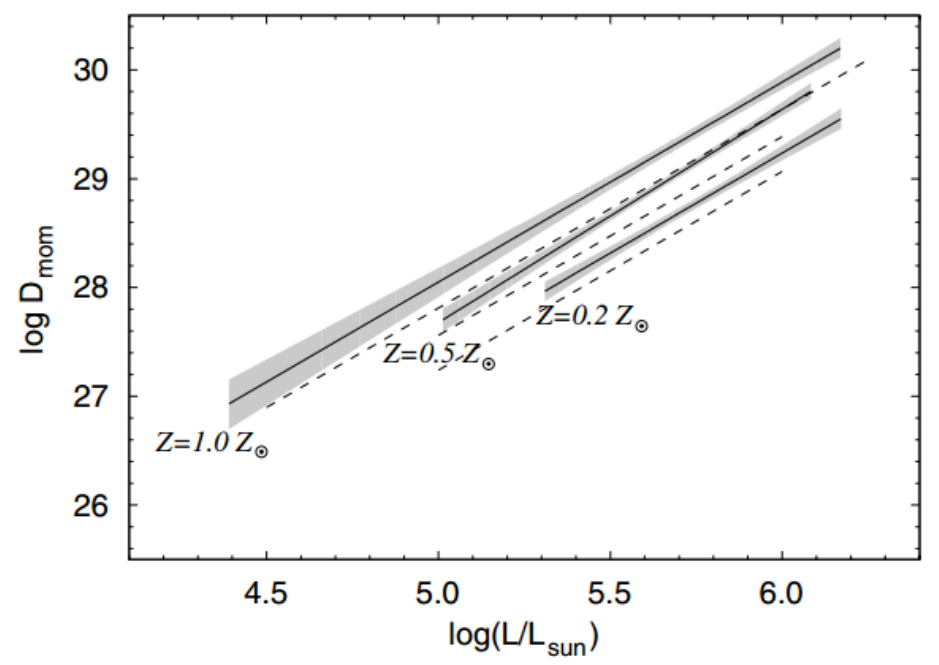

Figura 2.12. Comparación de la relación momento-luminosidad modificada en función de la metalicidad (Z). Las líneas punteadas y sólidas corresponden a predicciones teóricas Vink 2000 Vink et al. 2001) y a observaciones, respectivamente. De arriba hacia abajo se muestran las relaciones para la Vía Láctea, la Nube Mayor de Magallanes y la Nube Menor de Magallanes. El intervalo de confianza para las relaciones empíricas corresponde a las regiones grises. Extraído de Mokiem et al. 2007) 


\subsection{Vientos inhomogéneos}

Hasta aquí hemos desarrollado y descripto todos los parámetros y relaciones que nos brinda un modelo con ciertas hipótesis, por ejemplo, vientos con simetría esférica y homogéneos. Sin embargo, los avances en las diferentes técnicas de observación han traído como consecuencia, algunas evidencias que se apartan de las predicciones del modelo estándar. Es así que, en esta sección, dedicaremos especial atención a un fenómeno que está asociado con inhomogeidades (es decir, la existencia de regiones de sobre densidad) o grumos (clumps, su nombre en inglés) presentes en el viento. Este fenómeno conocido como clumping ha captado especial atención en las investigaciones relacionadas a las inhomogeneidades de los vientos de estrellas masivas en la última década.

\subsubsection{Inestabilidades hidrodinámicas de los vientos impulsados por radiación}

Las inestabilidades en el modelo de los vientos impulsados por radiación fueron sugeridas al mismo tiempo que se desarrollaba esta teoría por Lucy \& Solomon 1970. Los primeros análisis lineales de estabilidad mostraron que los vientos de las estrellas masivas eran inestables si se aplicaban perturbaciones en escalas cercanas o menores a la longitud de Sobolev MacGregor et al. 1979, Carlberg 1980, Owocki \& Rybicki 1984.

No se tardó mucho en confirmar directamente esta inestabilidad por medio de modelos de vientos dependientes del tiempo Owocki et al. 1988, Feldmeier 1995 Dessart \& Owocki 2005 que mostraron que un crecimiento no lineal de las inestabilidades conducían a rarefacciones de alta velocidad produciendo fuertes ondas de choque inversas. Una consecuencia directa de esto, es que el material se comprime generando grumos densos y estrechos que están separados por grandes regiones de menor densidad. De esta manera, estas estructuras características son la base teórica para nuestra comprensión e interpretación de lo que hoy conocemos como clumping (Sundqvist et al. 2012. La fig. 2.13 muestra una de estas estructuras típicas respecto a cómo se modifica la densidad y la velocidad en un modelo unidimensional perturbado a medida que el fluido se aleja de la estrella comparado con la predicción del modelo estándar.

Por otro lado, los choques producidos como consecuencia de las inestabilidades brindan una explicación para las emisiones y líneas espectrales observadas a muy altas energías por los satélites Copérnico Snow \& Morton 1976), Einstein Seward et al. 1979, Harnden et al. 1979), ROSAT (Berghoefer et al. 1997), XMM-Newton y Chandra Güdel \& Nazé 2009, Cohen et al. 2010). Cabe mencionar que esta explicación es la más aceptada ya que, alternativamente la presencia de campos magnéticos intensos también podría derivar en emisiones en rayos-X. Sin embargo, las estrellas supergigantes, en general, no poseen campos magnéticos de gran magnitud, aunque siempre existen objetos que escapan de estas generalidades Fossati et al. 2015. 

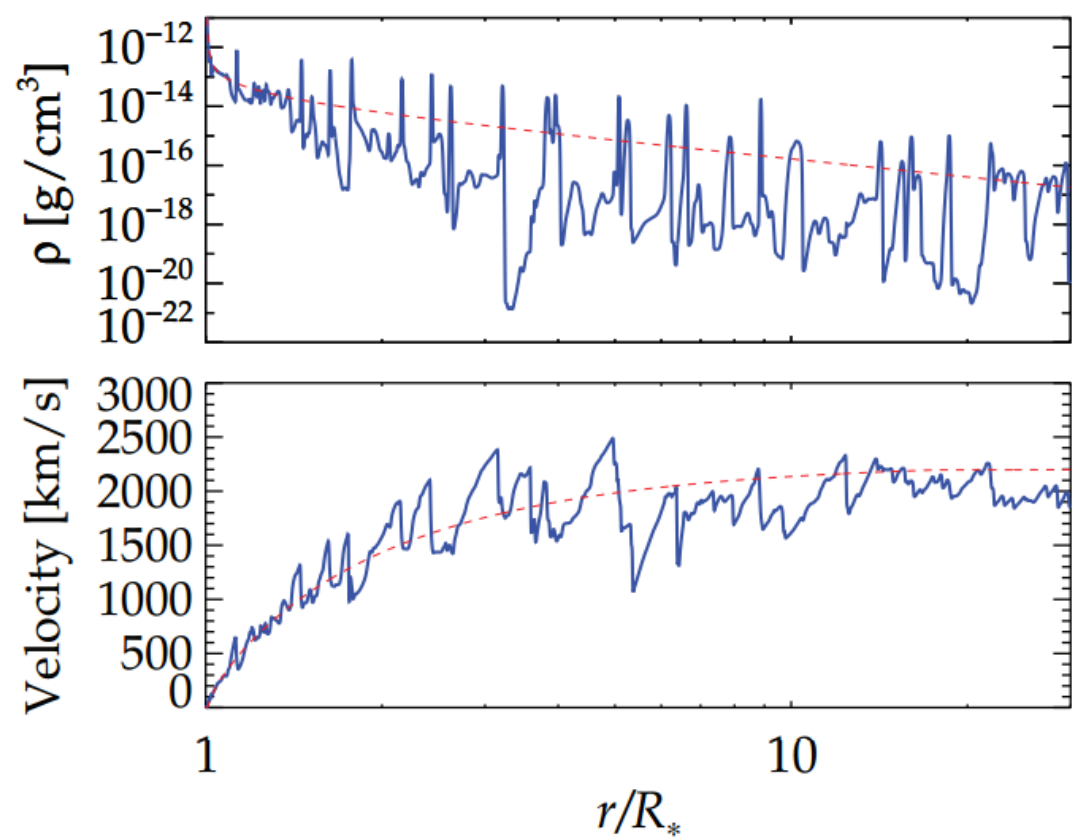

Figura 2.13. Densidad y velocidad del medio en función de la distancia a la estrella (arriba y abajo, respectivamente). La línea azul continua corresponde al medio perturbado y la línea punteada rojo al comportamiento estándar. Extraído de Sundqvist et al. 2012.

\subsubsection{Clumping}

El clumping tiene muchas implicancias en la interpretación de las observaciones, ya que los valores de la tasa de pérdida de masa determinados a partir de estudios en distintas regiones espectrales pueden ser muy diferentes si no se tiene en cuenta el factor de clumping $\left(f_{\mathrm{cl}}\right)$. Este factor es un nuevo parámetro en los códigos de transporte radiativo y es tenido en cuenta para descartar ciertas inconsistencias que tiene la teoría estándar de vientos impulsados por radiación. Este factor en particular rompe con la hipótesis de un viento suave y homogéneo, y sugiere la presencia de estructuras de pequeña escala, llamadas "clumps" o grumos, que poseen una densidad mayor que la densidad promedio del viento.

El factor de clumping permite abordar problemáticas que aparecen en la determinación de la tasa de pérdida de masa, en aquellos análisis que no consideran explícitamente la presencia de grumos. Una sobreestimación de la tasa de pérdida de masa se debe al simple hecho de que las líneas usadas para medir los parámetros del viento dependen de la densidad del mismo y la opacidad.

El mayor problema lo presenta la línea $\mathrm{H}_{\alpha}$ cuyo ancho equivalente aumenta con la densidad al cuadrado, mientras que las líneas de resonancia del UV dependen de $\rho$ linealmente Puls et al. 2008). Los valores de $\dot{\mathrm{M}}$ derivados a partir de líneas de diagnóstico que dependen de $\rho^{2}$ son menores en un factor $\sqrt{f_{\mathrm{cl}}}$ con respecto a valores determinados sin clumping. Massa et al. 2003 y Fullerton et al. 2004 2006 concluyeron que, para las estrellas O, los valores de pérdida de masa derivados de $\mathrm{H}_{\alpha}$ podrían sobreestimar $\dot{\mathrm{M}}$ en factores mayores 
a 100 mientras que Bouret et al. 2005), obtienen, para dos estrellas, factores entre 3 y 7. Repolust et al. 2005) y Puls et al. 2006 a partir del estudio del comportamiento del viento en diferentes regiones, es decir, analizando diferentes rangos espectrales, sugieren una estratificación espacial del clumping, sugiriendo que el factor de clumping no es constante a lo largo del viento. Aunque la muestra de objetos abarca sólo a las estrellas O, $\mathrm{Puls}$ et al. 2006) encuentran que para vientos densos (aquellos que presentan $\mathrm{H}_{\alpha}$ en emisión) el clumping es mayor en la región más interna que en la externa en un factor 4.1, mientras que para vientos delgados el comportamiento del clumping es similar en todas las regiones, con la excepción de algunos casos de tipos espectrales tempranos (B0 - B1).

En general el clumping no está del todo estudiado en las estrellas supergigantes B, y análisis similares a los de Repolust et al. 2005 y Puls et al. 2006 podrían llevarse a cabo. Pero teniendo en cuenta que estas estrellas son muy variables, para realizar un estudio más profundo de las propiedades del clumping, e incluso estudiar sus posibles variaciones, es necesario contar con numerosas observaciones en los diferentes rangos espectrales y que estas sean adquiridas en las mismas épocas.

La idea de la presencia de inhomogeneidades en el viento surge, por primera vez, para explicar la discrepancia observada entre el flujo teórico y el observado de la región H II en la Nebulosa de Orión Osterbrock \& Flather 1959. Y desde entonces las evidencias de este fenómeno han sido siempre indirectas, ya que su análisis es mediante el ajuste de líneas espectrales y/o del continuo de radio, a partir de la curva de luz de sistemas binarios, a partir de la variabilidad de las propiedadades polarimétricas, o a partir de la variabilidad espectral de ciertas líneas de emisión (ver detalles en el review de Puls et al. 2008). Por lo tanto, surge la necesidad de una observación directa del clumping, y esto sería mediante el uso de imágenes de muy alta resolución espacial para poder ver los grumos por separado.

\subsection{Modelos}

La pérdida de masa de una estrella influye no sólo en las propiedades cinemáticas y químicas del medio interestelar circundante sino también en la evolución de la propia estrella y su estructura atmosférica. Es por este motivo, que para poder comprender las propiedades de los vientos, éstos necesitan ser correctamente modelados, utilizando un código que resuelva la ecuación de transporte para obtener los perfiles de líneas teóricos, y donde $\dot{\mathrm{M}}$, $v_{\infty}$ y $\beta$ (relacionada con la ley de velocidades, ver secc. 2.9 son, entre otros, parámetros de entrada. Por medio de la variación de estos tres parámetros, se pueden realizar ajustes óptimos de perfiles de líneas que caracterizan a un viento y obtener así los "parámetros observables del viento", que son los datos de salida del proceso de ajuste. Adicionalmente a estos parámetros que nos permitirán realizar una descripción del viento, existen otros como la temperatura efectiva $\left(\mathrm{T}_{\text {ef }}\right)$, la gravedad superficial $(\log g)$ y las abundancias, que afectan el proceso radiativo, y que debemos tener en cuenta a la hora de modelar y ajustar un perfil de línea teórico a la observación.

Una vez que los parámetros derivados de las observaciones han sido obtenidos, tenemos que ser conscientes de que ellos son el resultado de códigos numéricos basados en varias hi- 
pótesis y que dependiendo del grado de sofisticación del mismo, pueden producir resultados que pueden diferir mucho de un código a otro.

La mejora más relevante en los códigos del campo de la espectroscopía cuantitativa de estrellas masivas fue la incorporación de los efectos de line-blocking/blanketing ${ }^{19}$ y backwarming ${ }^{20}$ a los modelos de atmósferas fuera del equilibrio termodinámico local (NLTE, por sus siglas en inglés). Esta posibilidad de incluir los efectos de miles de líneas de metales se llevó a cabo gracias a los nuevos avances tecnológicos, en cuanto a facilidades computacionales se refiere. Existen varios códigos que modelan la atmósfera estelar y calculan el estado hidrodinámico del viento conjuntamente con el transporte de radiación. Por ejemplo, CMFGEN (Hillier \& Miller 1998), WM-BASIC (Pauldrach et al. 2001), FASTWIND Santolaya-Rey et al.1997, entre otros. Entre los modelos con mayores logros encontramos a los obtenidos con FASTWIND, el cual está orientado a que la ejecución del código sea rápida y sencilla. Usaremos este código para realizar el ajuste de nuestras observaciones, por lo que, a continuación, describiremos sus principales características.

\subsubsection{FASTWIND (Fast Analysis of STellar atmospheres with WINDs)}

Para describir las características de los vientos es necesario disponer de un modelo que resuelva adecuadamente la atmósfera en movimiento, y que incluya no sólo el comportamiento de la presión $(\mathrm{p})$, temperatura $(\mathrm{T})$, densidad $(\rho)$ y velocidad $(\mathrm{v})$ en función de la distancia a la estrella, sino que prediga también cómo es el espectro emergente. Esto último es casi fundamental porque nos va a permitir comparar un espectro sintético con el espectro observado.

FASTWIND ha sido diseñado para estudiar las estrellas $\mathrm{O}, \mathrm{B}$ y $\mathrm{A}$ de diferentes luminosidades y sus vientos. Su primera versión fue desarrollada por Santolaya-Rey et al. (1997).

Es importante diferenciar dos grupos diferentes de elementos químicos tratados en el código: los explícitos y los de fondo o background. Los elementos explícitos son principalmente el $\mathrm{H}$ y el He, pero pueden extenderse a C, N, O, Si y Mg, y ellos son tratados con muy alta precisión, es decir, mediante modelos atómicos detallados en NLTE. Los iones de background, por otro lado, son tenidos en cuenta con aproximaciones estadísticas para los efectos de bloqueo y blanketing de las líneas.

FASTWIND sigue el concepto de "modelo unificado de atmósfera", es decir, considera una transición suave entre la fotosfera y el viento y se aplica a sistemas estacionarios con simetría esférica y libres de campos magnéticos.

El modelo de fotosfera está en pseudo equilibrio hidrostático y se obtiene a partir de modelos plano-paralelos (corregidos por extensión y esfericidad) y "conectado" al viento

\footnotetext{
${ }^{19}$ Bloqueo/disminución del continuo espectral de una estrella debido a la gran cantidad de líneas de absorción muy poco espaciadas e indefinidas. Este efecto es particularmente notable en la región UV de las estrellas calientes. En el caso de estrellas frías, éstas poseen en sus atmósferas muchos átomos y moléculas que tienden a absorber fotones con longitudes de onda cortas y reemitir en el rojo e IR.

${ }^{20}$ Redistribución de la energía en otras regiones espectrales.
} 
(parte más externa de la atmósfera) por un punto de transición en la región sónica. La estructura de temperatura y la aceleración radiativa del continuo son aproximadas para simular el equilibrio radiativo (para evitar cualquier otro proceso de iteración que consumiría mucho tiempo, pero sin perder un alto grado de precisión en los cálculos).

El viento se calcula con una ley de velocidades ad-hoc tipo $\beta$, de la forma

$$
v(r)=v_{\infty}\left(1-b \cdot r / r_{\star}\right)^{\beta}
$$

donde

$$
b=1-\left[v_{0} / v_{\infty}\right]^{1 / \beta}
$$

El resto de las cantidadas relevantes (densidad, presión, etc.) se obtienen de las relaciones definidas en la sección 2.5.2

De esta manera el viento queda especificado por la tasa de pérdida de masa, la velocidad terminal y el parámetro $\beta$, y la fotosfera por $\mathrm{T}_{\mathrm{ef}}, \log \mathrm{g}, \mathrm{R}_{\star} \mathrm{y}$ la abundancia.

Otras características importantes del código para calcular la solución aproximada a las ecuaciones de transporte radiativo son:

- La solución es calculada sobre una grilla radial de las variables termodinámicas de la atmósfera: temperatura, densidad, poblaciones atómicas, etc..

- El cálculo del transporte de radiación del continuo y la línea es tratado de forma independiente.

- Adopta ensanchamiento por efecto "Stark" (campo eléctrico): típicamente este ensanchamiento para $\mathrm{H}$ y He II es dominante para $\log \left(N_{e}\right)>12$. El código comienza a considerarlo para $\log \left(N_{e}\right)>10.5$ y para bajas densidades sólo considera el ensanchamiento por efecto Doppler (usado en esta tesis).

- Trata consistentemente el scattering electrónico (si se requiere), el cual produce dos efectos:

1. Los fotones que son emitidos en la atmósfera se desplazan hacia el rojo, por la acumulación del efecto de scattering electrónico en el viento, lo que resulta en una extendida ala roja en los perfiles de línea.

2. Debido a la gran diferencia entre la velocidad térmica de los electrones y iones, los fotones dispersados por electrones pueden ser redistribuidos en frecuencia. Este efecto produce alas más extensas a ambos lados del perfil y es muy probable que sean observados en las alas de $\mathrm{H}_{\alpha}$ de supergigantes A y B.

Resumiendo, las características de este código son:

- Código unificado, es decir, calcula una fotosfera y un viento de manera consistente y en un mismo programa.

- Ley $\beta$ para el viento. 
- En las primeras 20 iteraciones la ecuación de transporte se resuelve aplicando la aproximación de Sobolev (ver secc. 2.5.3) y luego se emplea el marco de referencia del fluido.

- Algoritmo rápido para calcular los perfiles de las líneas.

En particular, para los cálculos realizados en este trabajo se utilizó la versión v10.1.7 de FASTWIND, cedida gentilmente por el Dr. Joachim Puls (Universidad de Munich).

\subsection{BSGs: discrepancias con la teoría estándar}

Las relaciones obtenidas en la teoría clásica o m-CAK (ecs. 2.26 2.27 y 2.29) nos brindan una variedad interesante de nuevas posibilidades de diagnóstico:

1. Deteminar distancias solamente con tasas de pérdida de masa y velocidades terminales obtenidas directamente de los perfiles de líneas observados.

2. Con la relación entre $v_{\infty}$ y la velocidad de escape, $v_{\text {esc }}$, es posible predecir la energía del viento y el momento transferido al medio interestelar, no sólo de una estrella aislada, sino también de cúmulos y asociaciones de estrellas calientes.

3. Se podrían usar las estimaciones de la tasa de pérdida de masa, energía y momento del viento, para precisar los caminos evolutivos estelares adecuados.

Esta teoría ha sido muy exitosa al explicar el viento de las estrellas supergigantes $\mathrm{O}$ y estrellas WR. Sin embargo, se encuentran discrepancias cuando se compara la teoría CAK con las observaciones de los parámetros del viento en las supergigantes B Crowther et al. 2006a Markova et al. 2008a. Usando la técnica de síntesis espectral estos autores encuentran que el viento estelar de las supergigantes B puede ser modelado con valores de $\beta$ que se encuentran entre 1 y 3 , siendo estos valores muchos mayores a los esperados con la teoría $\operatorname{CAK}(\beta=0.8$, Abbott 1982$)$ ).

Curé 2004 encontró que para velocidades de rotación mayores que 0.75 veces la velocidad de rotación crítica, aparece una nueva solución hidrodinámica, que da lugar a un flujo de materia con una velocidad monótonamente creciente, pero con una aceleración menor en la región subcrítica, que la que predice la teoría m-CAK (soluciones rápidas). Esta nueva solución (solución $\Omega$-lenta) trae como consecuencia un aumento de la densidad $\rho$ del medio con la distancia. Esto lleva a suponer que objetos que presentan alta rotación podrían tener dos regímenes de velocidades diferentes y simultáneos, dependiendo de la latitud estelar (ya que la fuerza centrífuga tiene una dependencia con v sen i). Así, a partir de una determinada latitud, la solución rápida dejaría de existir y surgiría una nueva solución (solución lenta) que generaría un viento más denso y lento. La existencia de estas nuevas soluciones fue confirmada por Madura et al. 2007) empleando un código hidrodinámico 1-D dependiente del tiempo y podría explicar el origen de los discos observados en estrellas supergigantes B $[\mathrm{e}]$ Curé et al.2005. 


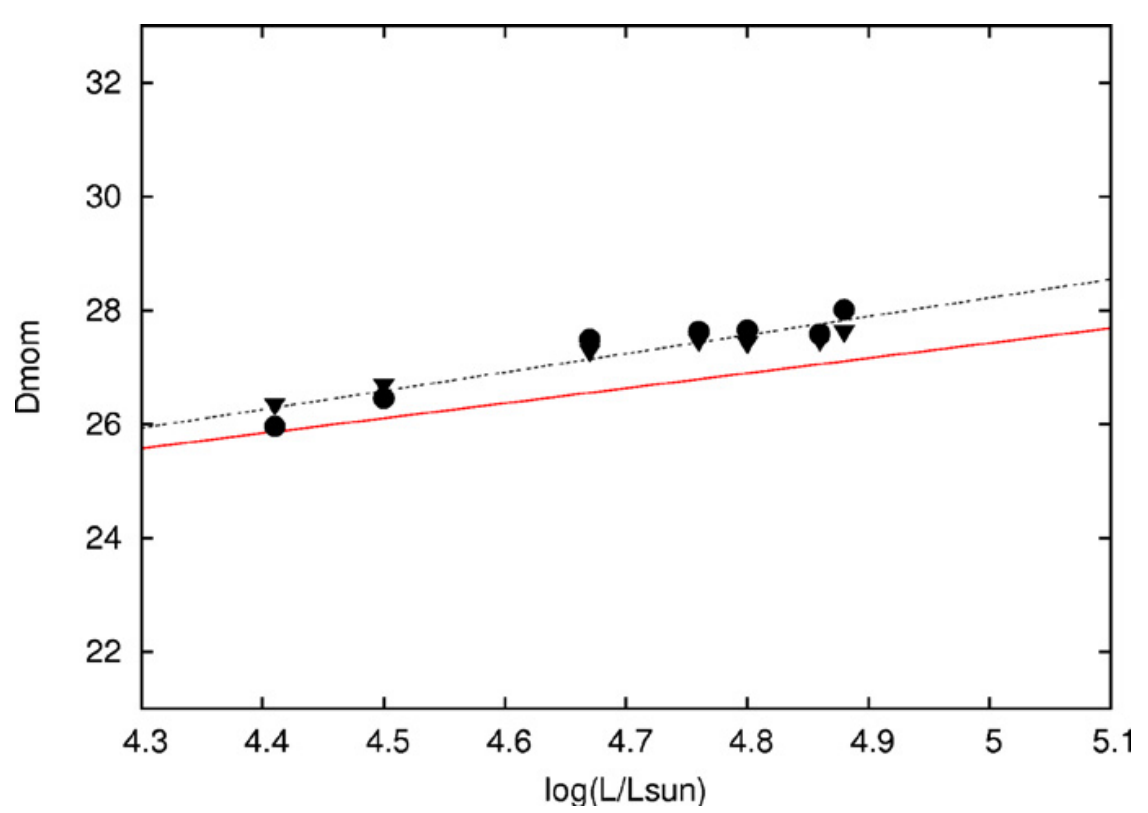

Figura 2.14. Para estrellas supergigantes A, la relación momento-luminosidad teórica (línea punteada) se ajusta muy bien con las observaciones (línea roja). Los puntos y triángulos negros corresponden a distintos valores teóricos de la fuerza de radiación. Extraído de Curé et al. 2011.

Posteriormente, Curé et al. 2011 han estudiado la topología de las soluciones hidrodinámicas sobre un espacio de parámetros de la fuerza de radiación (principalmente el parámetro $\delta$ que describe el cambio de la ionización a lo largo del viento) encontrando que en aquellos casos donde el viento presenta variaciones de la ionización con la distancia es posible obtener un régimen de velocidad lento, aún cuando la rotación estelar es baja (e incluso cero). Las nuevas soluciones (soluciones $\delta$-lenta) predicen cocientes entre la velocidad terminal del viento y la de escape similar a los valores observados en las supergigantes A, hecho que no puede ser explicado en el contexto de la teoría clásica m-CAK. Las nuevas soluciones $\delta$-lentas ajustan también la relación empírica momento-luminosidad de estos objetos, describiendo así las características de los vientos débiles, como muestra la fig. 2.14 y fig. 2.15, respectivamente.

\subsection{Propiedades de los vientos estelares}

Además de explorar $\mathrm{v}_{\infty}$ y $\dot{\mathrm{M}}$ a partir de la línea de $\mathrm{H}_{\alpha}$ (ver secc. 2.3), también pueden determinarse otros dos parámetros: el parámetro $\beta$ y el invariante de profundidad óptica (Q).

El parámetro $\beta$ está ligado al campo de velocidades del fluido y surge, como vimos en la secc. 2.5.2, a partir de una aproximación para resolver las ecuaciones planteadas en la teoría clásica. Este valor nos da información acerca del campo de velocidades que gobierna a las partículas del viento, es decir, cómo se comporta la velocidad a medida que las partículas 


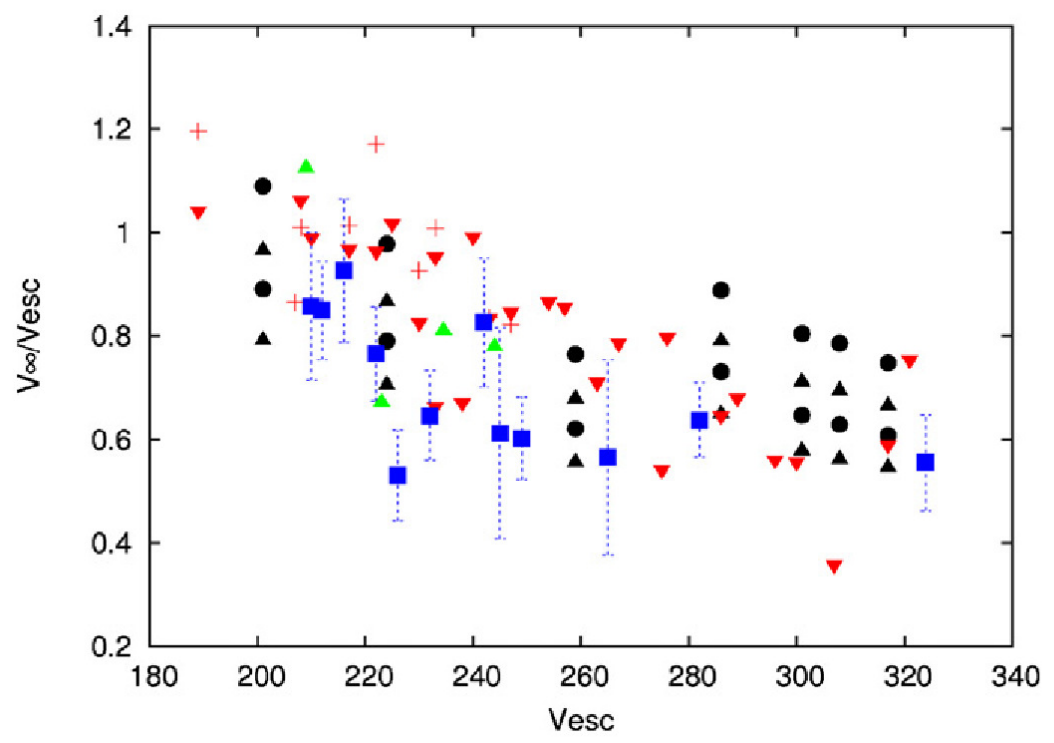

Figura 2.15. Para estrellas supergigantes A, la teoría de vientos lentos predice una relación lineal con pendiente negativa para describir la relación entre la velocidad terminal del viento y la velocidad de escape de la estrella. Los puntos y triángulos negros corresponden a soluciones hidrodinámicas diferentes para las direcciones polares y ecuatoriales de estrellas supergigantes A, respectivamente y los símbolos rojos y azules son distintos datos observacionales. Extraído de Curé et al. 2011.

se alejan de la estrella o si éstas son aceleradas principalmente en la base del viento o no. La teoría predice valores de $\beta$ entre 0.5 y 0.8 Castor et al. 1975, Abbott 1982, pero el uso de la "ley $\beta$ " se ha extendido para representar los vientos de las estrellas tempranas, cubriendo valores que van desde 0.5 y que pueden llegar a valores cercanos a 3 (o incluso un poco mayores) (Kudritzki et al. 1999, Lefever et al. 2007).

Por otro lado, en lugar de medir $\dot{\mathrm{M}} \mathrm{y} \mathrm{v}_{\infty}$ individualmente, lo que usualmente se determina en los trabajos donde se realizan grandes grillas de cálculos, es el parámetro de la fuerza del viento $\mathrm{Q}=\dot{\mathrm{M}} /\left(\mathrm{R}_{\star} \mathrm{v}_{\infty}\right)^{1,5}$ Puls et al. 1996. De esta manera no se obtiene información directa de la tasa de pérdida de masa a partir de los ajustes de perfiles teóricos con los observados, sino que ésta se determina por medio del parámetro Q. La razón por la cual se elige como valor medido a $Q$ en lugar de $\dot{M}$, es que diferentes combinaciones de $\dot{M}$, $\mathrm{v}_{\infty} \mathrm{y} \mathrm{R}_{\star}$ producen el mismo valor de $\mathrm{Q}$, generando los mismos perfiles teóricos de $\mathrm{H}_{\alpha}(\mathrm{Puls}$ et al. 1996. El resto de las líneas permanecen casi inalteradas Puls et al. 2005. Por lo tanto, una vez que se encuentra dentro de la grilla un perfil sintético (un valor de Q) que ajuste la observación, $\mathrm{y}_{\infty} \mathrm{y} \mathrm{R}_{\star}$ son conocidos, se determina $\dot{\mathrm{M}}$. En esta tesis no hemos realizado grillas de modelos con pasos determinados para los diferentes parámetros, sino que hemos modelado individualmente cada objeto, con lo que los parámetros medidos del viento son directamente $\dot{\mathrm{M}}, \mathrm{v}_{\infty} \mathrm{y} \beta$.

Es necesario remarcar que para vientos ópticamente delgados, o poco densos, por lo general la línea de $\mathrm{H}_{\alpha}$ se encuentra en absorción pura, lo cual hace imposible determinar 


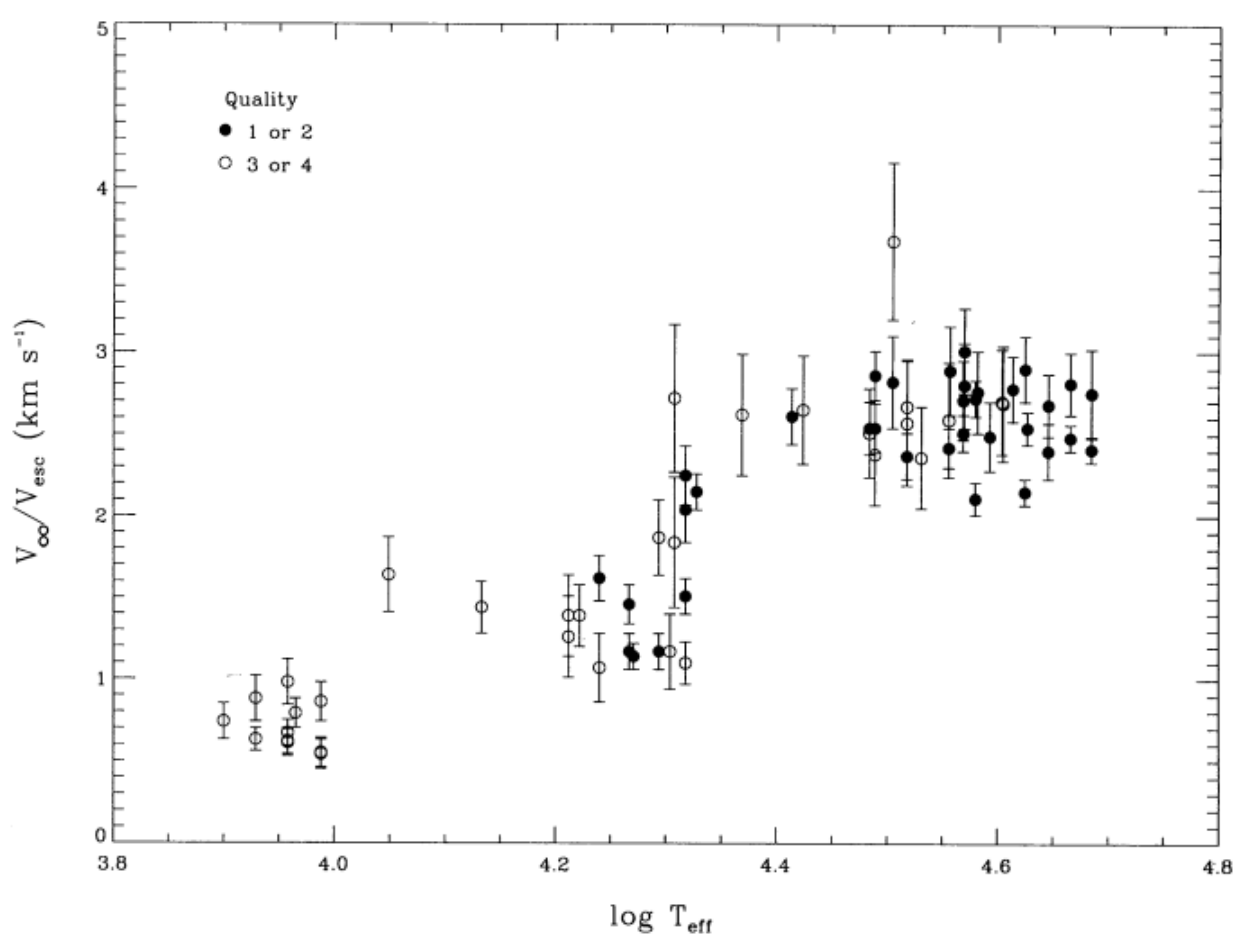

Figura 2.16. Salto de biestabilidad en $\sim 21000 \mathrm{~K}\left(\sim \log \left(\mathrm{T}_{\mathrm{ef}}\right)=4.32\right)$. Extraído de Lamers et al. 1995.

$\mathrm{v}_{\infty}$

A modo de comentario final, es necesario tener en cuenta que a la hora de modelar los vientos de las estrellas masivas, se van variando los tres parámetros fundamentales $\left(\dot{\mathrm{M}}, \mathrm{v}_{\infty}\right.$ y $\beta$ ) hasta conseguir un buen ajuste.

\subsubsection{Salto de biestabilidad}

Un fenómeno muy particular que evidencia cambios drásticos en las propiedades de los vientos de las estrellas de tipo espectral B1 es el "salto de biestabilidad". Lamers et al. 1995 encontraron que, a una temperatura efectiva de $21000 \mathrm{~K}$ (correspondiente al tipo espectral B1), el cociente $\mathrm{v}_{\infty} / \mathrm{v}_{\text {esc }}$ cae un factor $\sim 2$ desde $\mathrm{v}_{\infty} / \mathrm{v}_{\text {esc }} \sim 2.6$ a $\sim 1.3$, desde la región correspondiente a altas temperaturas hasta la región correspondiente a temperaturas más frías (ver fig. 2.16), y atribuyeron este comportamiento a un cambio en la naturaleza de las líneas que producen la presión de radiación.

Si bien Lamers et al. 1995 fueron los primeros en reportar este comportamiento, Crowther et al. 2006a) observaron que más que un "salto" en B1 es más bien una tendencia gradual de $\mathrm{v}_{\infty} / \mathrm{v}_{\text {esc }} \sim 3.4$ para supergigantes B0 - B0.5 $\left(\mathrm{T}_{\text {ef }}>24000 \mathrm{~K}\right) \mathrm{a} \mathrm{v}_{\infty} / \mathrm{v}_{\text {esc }} \sim 2.5$ para B0.7 - B1 $\left(20000 \mathrm{~K}<\mathrm{T}_{\mathrm{ef}}<24000 \mathrm{~K}\right)$ y a $\mathrm{v}_{\infty} / \mathrm{v}_{\mathrm{esc}} \sim 1.9$ para estrellas supergigantes B1.5 - B3 con temperaturas efectivas menores a 20000 K. Sin embargo, Markova \& Puls 2008, debido a la gran cantidad de nuevas observaciones, proponen atacar este problema 


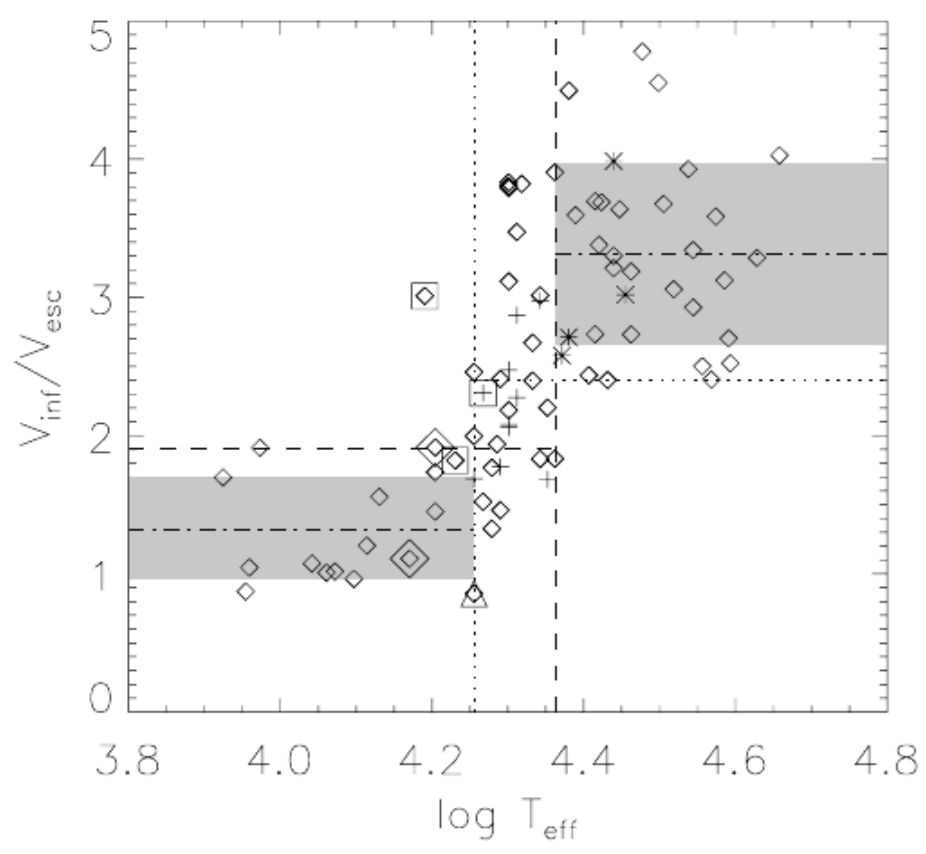

Figura 2.17. Salto de biestabilidad de una muestra significante de estrellas supergigantes O, B y A galácticas. Extraído de Markova \& Puls 2008.

definiendo la posición del salto a partir de valores medios de $\mathrm{v}_{\infty} / \mathrm{v}_{\text {esc }}$ de supergigantes $\mathrm{O}$, $\mathrm{B}$ y $\mathrm{A}$ reportados en la literatura. El resultado puede verse en la fig. 2.17, donde pueden identificarse claramente dos regiones de temperaturas con diferentes $\mathrm{v}_{\infty} / \mathrm{v}_{\text {esc }}$ conectadas por una región de transición. Para $\mathrm{T}_{\text {ef }}>23000 \mathrm{~K}$, estos autores encuentran que $\mathrm{v}_{\infty} / \mathrm{v}_{\text {esc }}$ $\simeq 3.3 \pm 0.7$, mientras que para $\mathrm{T}_{\mathrm{ef}}<18000 \mathrm{~K}, \mathrm{v}_{\infty} / \mathrm{v}_{\mathrm{esc}} \simeq 1.3 \pm 0.4$ (líneas a rayas $\mathrm{y}$ puntos horizontales en la fig. 2.17). También definen que el "salto" ocurre en $\mathrm{T}_{\mathrm{ef}} \simeq 20000$ $\mathrm{K}$, pero sin embargo extienden la región de biestabilidad del gráfico (delimitada por la líneas verticales) hasta $T_{\text {ef }}=23000 \mathrm{~K}$ ya que a esa temperatura aún es posible encontrar cocientes bajos de $\mathrm{v}_{\infty} / \mathrm{v}_{\text {esc }}$.

Por otro lado, la contraparte teórica de este fenómeno es explicada por Vink et al. (1999) que predice un salto alrededor de $25000 \mathrm{~K}$ hacia temperaturas más frías donde la pérdida de masa aumenta un factor 5 y la $\mathrm{v}_{\infty}$ disminuye un factor 2 , y atribuyen este fenómeno a la recombinación de FeIV en Fe III. En cambio, Markova \& Puls 2008 encontraron observacionalmente que un decrecimiento en $\mathrm{v}_{\infty}$ en la región de biestabilidad no es compensado por un incremento en $\dot{\mathrm{M}}$ y que es más probable que $\dot{\mathrm{M}}$ decrezca en paralelo con $\mathrm{v}_{\infty} / \mathrm{v}_{\mathrm{esc}}$.

\subsubsection{Variabilidad}

Algunos descubrimientos revelaron que los espectros de las estrellas OB supergigantes no permanecen invariantes en el tiempo, sino que sufren diferentes variaciones producidas 
por cambios en la estructura interna y en el viento. Como consecuencia, las líneas espectrales cambian tanto su intensidad, como su forma y simetría. Los fenómenos más conocidos y estudiados son las "absorciones de alta velocidad" y las "componentes de absorción discretas" (HVAs y DACs por sus siglas en inglés, respectivamente).

Las HVAs son absorciones que se desarrollan a lo largo de un rango de velocidades y que pueden estar corridas simultáneamente hacia el rojo y al azul. Son extremadamente variables y las líneas ópticas de recombinación son las más afectadas. La fig. 2.18 muestra este fenómeno en la línea de $\mathrm{H}_{\alpha}$ de HD 199478 (B8Ia), donde puede apreciarse claramente cómo estas intensas absorciones se hacen presentes y afectan por completo la línea espectral, siendo más intensas en una época que en otra. Si bien el motivo de por qué se producen estos fenómenos aún no es del todo claro, las principales hipótesis van desde fenómenos asociados con pérdida de masa en la base del viento y que se propagan a través de éste, efectos de ionización en la envoltura, o hasta efectos combinados de rotación con regiones de sobredensidad en la línea de la visual (Kaufer et al. 1996).

Las DACs, por su parte, se observan en líneas de resonancia del rango UV. Estas DACs están superpuestas a las absorciones de los perfiles P Cygni no saturados y son identificadas a bajas y altas velocidades. La principal característica es que "migran" desde la longitud de onda más larga hacia la más corta de la componente en absorción de los perfiles P Cygni Prinja \& Howarth 1988 Massa et al. 1995: Kaper et al. 1997, Prinja et al. 2002 y son muy angostas cuando se acercan a la velocidad terminal del viento (Kaper et al. [1999). En la fig. 2.19 se puede observar no sólo la presencia de estas componentes en las líneas de Mg II $\lambda 2795,2802 \AA$ en Rigel, sino también su variación entre los años 1980 y 1988. La flechas marcan diferentes DACs y puede verse que en 1980 se distinguen claramente 5 de ellas, mientras que en 1988 sólo una está presente.

Las principales características de las DACs, es decir su recurrencia y su aceleración pueden ser explicadas mediante la existencia de regiones corrotantes de interacción (CIR, Mullan 1986. Los primeros en proponer la presencia de estas CIRs en el viento de las estrellas O con rotación fueron Cranmer \& Owocki 1996. Mediante simulaciones hidrodinámicas en dos dimensiones de las inhomogeneidades azimutales de los vientos impulsados por radiación, estos autores encuentran que, realizando perturbaciones en la fuerza de radiación cerca de la superficie estelar, se desarrollan corrientes de altas densidades y bajas velocidades (compresiones) y viceversa (rarefacciones), como puede apreciarse en la fig. 2.20 .

Lo que aún permanece incierto es el origen físico de estas perturbaciones, ya que pueden deberse a la presencia de campos magnéticos superficiales o pulsaciones no radiales. Pero más allá de la formación de las CIRs, lo interesante es que el viento supersónico intrínseco (no perturbado) de la estrella impacta con las regiones de sobredensidad de las CIRS produciendo un choque inverso no lineal. Respecto al marco de referencia del viento esta "anomalía" se propaga hacia adentro, pero como la velocidad del choque en general es apenas menor que la velocidad del viento, éste se desplaza lentamente hacia afuera respecto de la estrella. El resultado de estas propagaciones son pliegues (o "kinks") que serían las responsables de las absorciones observadas en los perfiles P Cygni no saturados, es decir, de las DACs Cranmer \& Owocki 1996. 


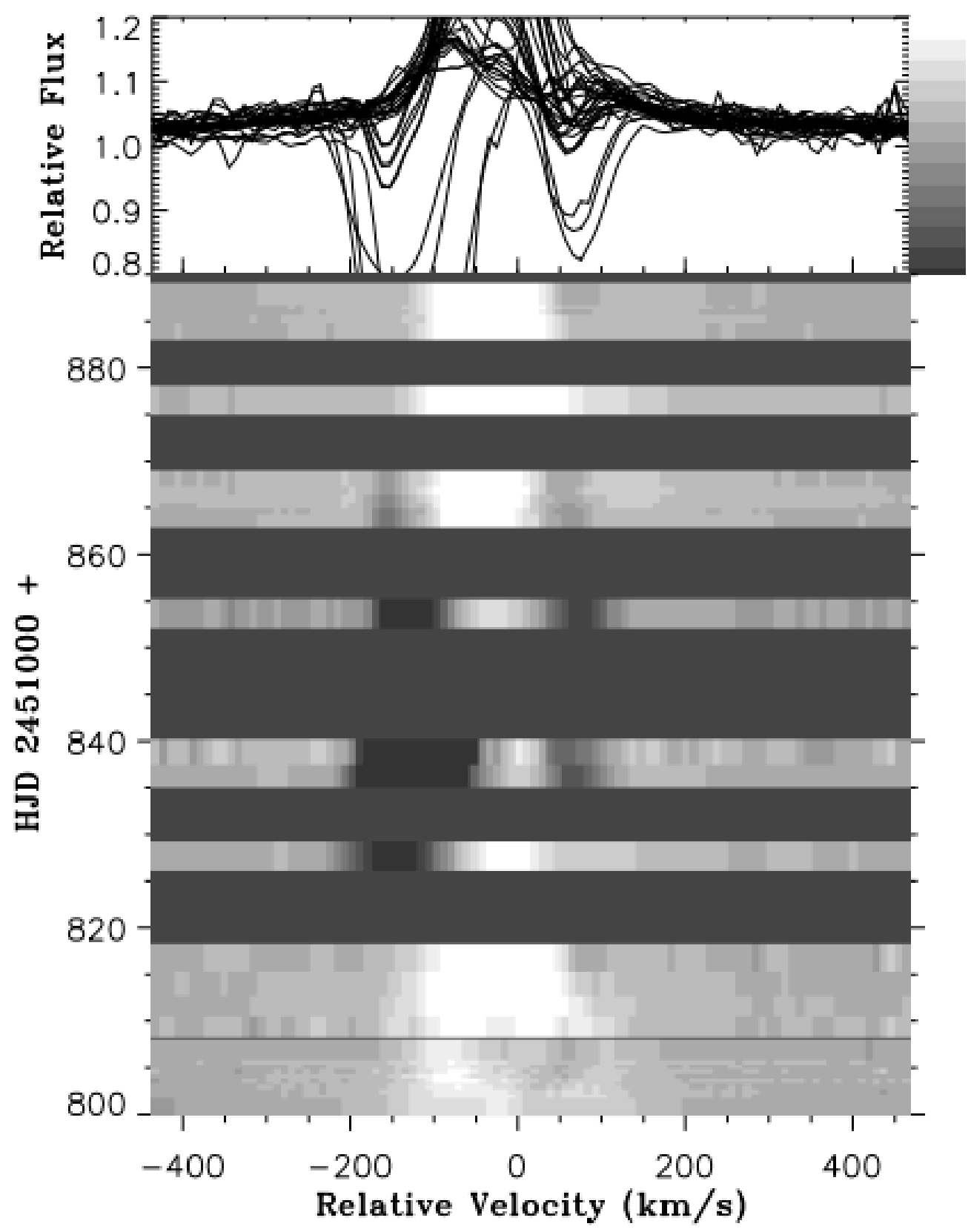

Figura 2.18. Evento de HVA observado en $\mathrm{H}_{\alpha}$ en HD 199478 (B8 Iae), extraído de Markova et al. 2008b. Ambos paneles muestran las variaciones del perfil para distintas épocas. Las absorciones del panel superior se corresponden a las regiones oscuras del panel inferior, mientras que las emisiones están asociadas con las regiones brillantes. 


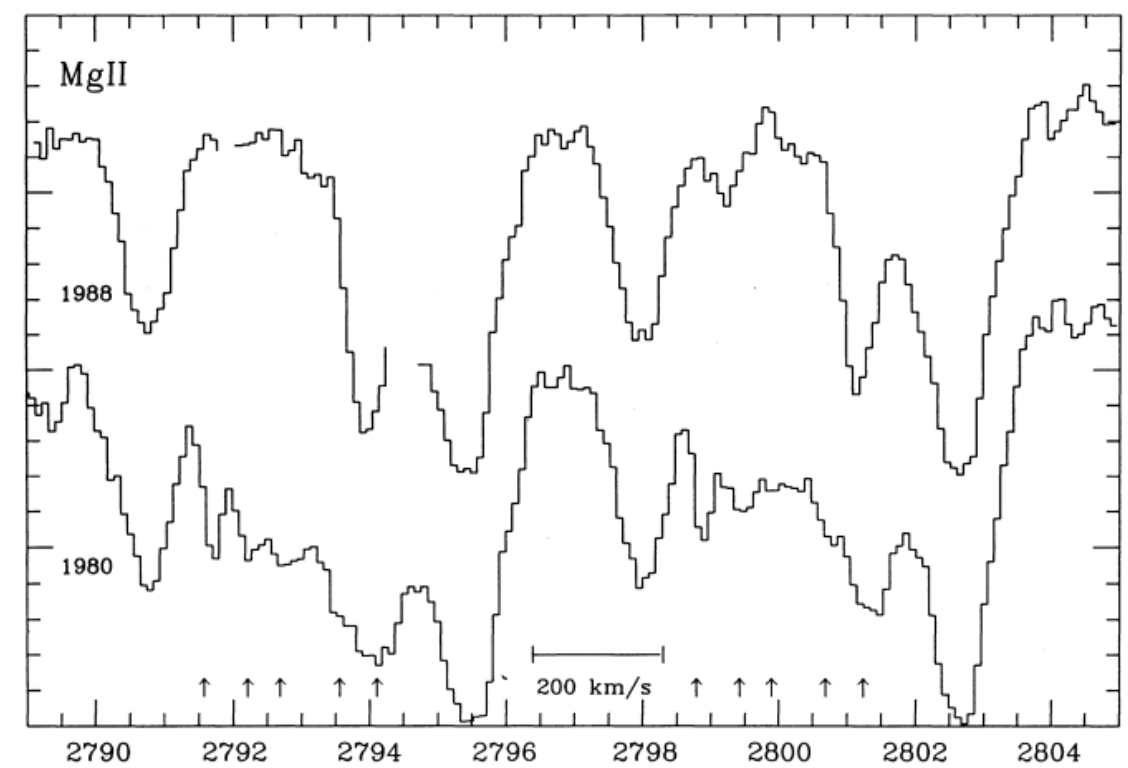

Figura 2.19. Espectro que muestra las DACs asociadas a la línea de Mg II $\lambda 2795,2802 \AA$ de la estrella Rigel (B8 Iae), extraído de Bates et al. (1988). El eje de las ordenadas y el de las abscisas representan el flujo relativo y la longitud de onda, respectivamente. Las flechas marcan la presencia de diferentes DACs.

Si bien muchas de las BSGs están catalogadas como variables IA, variables poco estudiadas de tipo espectral temprano (Lefèvre et al. 2009), la mayoría pertenece al grupo de las variables $\alpha$-Cygni (por su estrella prototipo). Abarcan las estrellas OBA masivas, de alta luminosidad, que poseen microvariaciones fotométricas $\left(\Delta \mathrm{Mag} \leq 0^{m} .15\right.$, Kholopov et al. 1996) y también variaciones espectroscópicas, tanto en la fotosfera (ver fig. 2.21) como en el viento (Stahl et al. 1995, Kaufer et al. 1996, 1997).

\subsubsection{Posibles mecanismos de variabilidad}

En la última década, las pulsaciones han cobrado protagonismo al tratar de explicar las variaciones que ocurren en la fotosfera y en el viento de estrellas masivas Aerts et al. 2009a Saio et al.2013a: Godart et al. 2017, analizando cómo se verían afectadas las diferentes líneas espectrales dependiendo del tipo de pulsación que esté presente (ver fig. 2.22).

Otro factor a tener en cuenta para la variabilidad es el campo magnético. Son pocas las estrellas BSGs detectadas con campos magnéticos ya que éstos son muy débiles (centenares de Gauss), pero sin embargo, algunas mediciones revelaron no sólo que presentan este campo, sino que también pueden ser variables. Tal es el caso de $\epsilon$ CMa (B1.5II) cuyo campo magnético promedio es de 149 G (Morel et al. 2008). Con el objetivo de estudiar con más profundidad los campos magnéticos en las estrellas B, varios estudios observacionales han sido llevados a cabo en los últimos años, como son los casos de The B field in OB Stars (BOB) Survey Kholtygin et al.2015) y The Magnetism in Massive Stars (MiMeS) Wade 


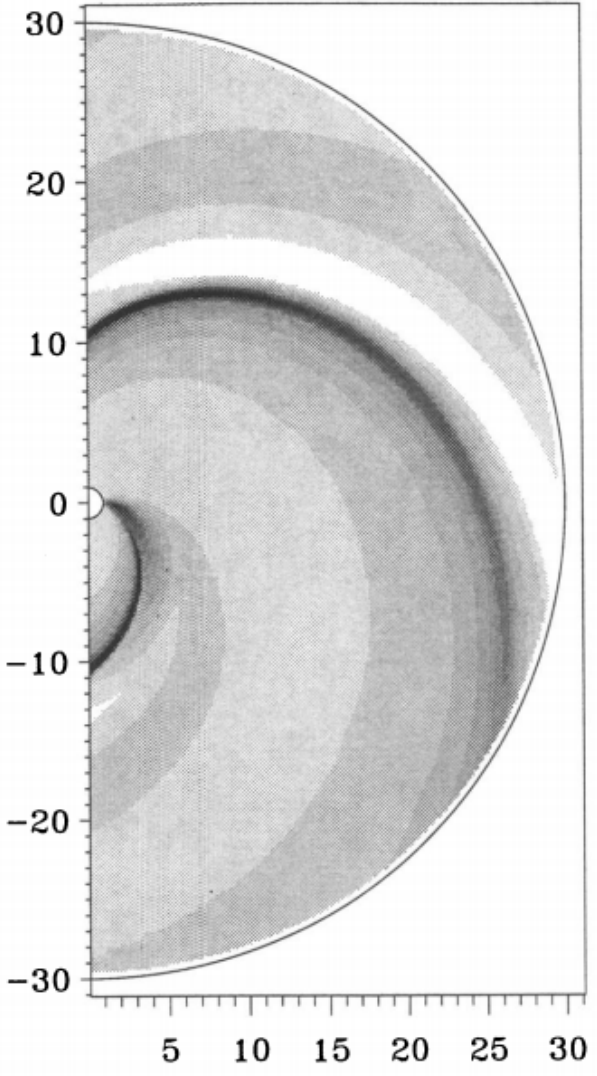

(a)

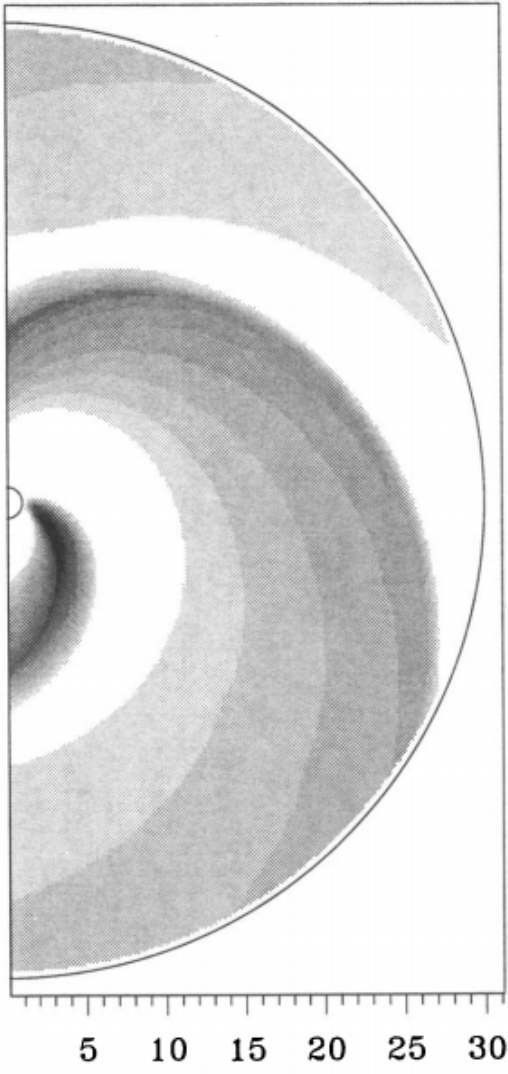

(b)

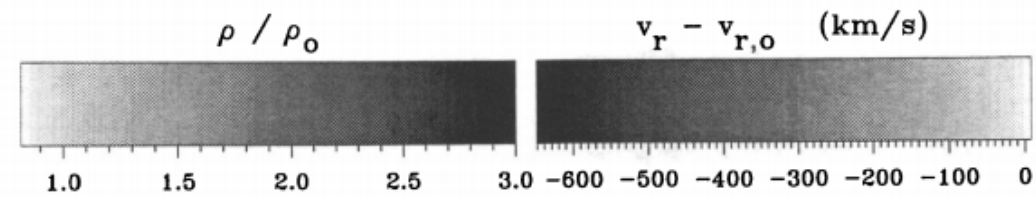

Figura 2.20. Modelo de estructura de CIR de Cranmer \& Owocki 1996. Los paneles (a) y (b) muestran las distribuciones de densidad y velocidad de las CIRs, respectivamente. 

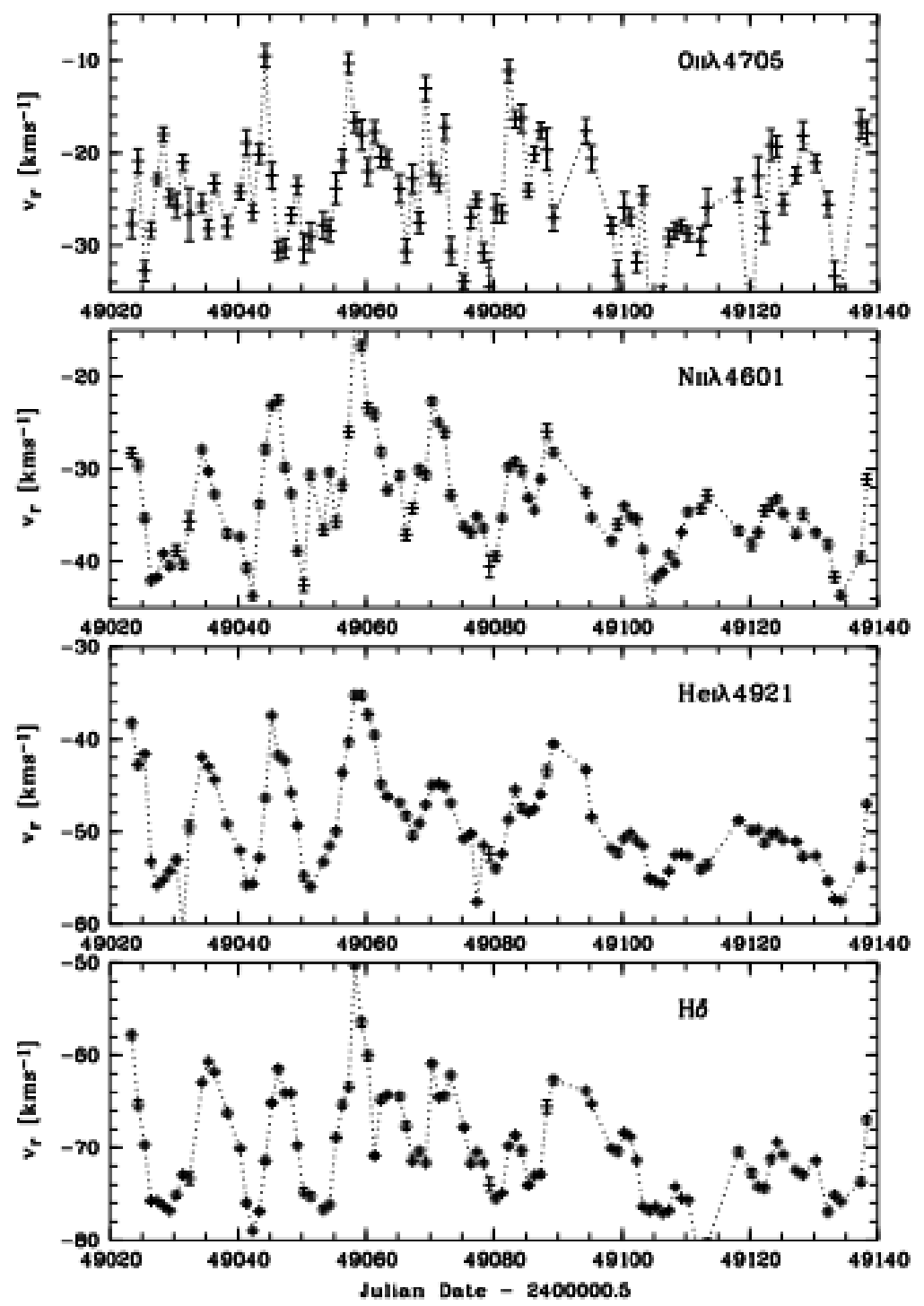

Figura 2.21. Variación de velocidades radiales proyectadas de las diferentes líneas fotosféricas O II $\lambda$ 4705, N II $\lambda$ 4601, He I $\lambda$ 4921, y H $\delta$ de la estrella $\zeta^{1}$ Sco (B1.5Ia+ Rivinius et al. 1997. 


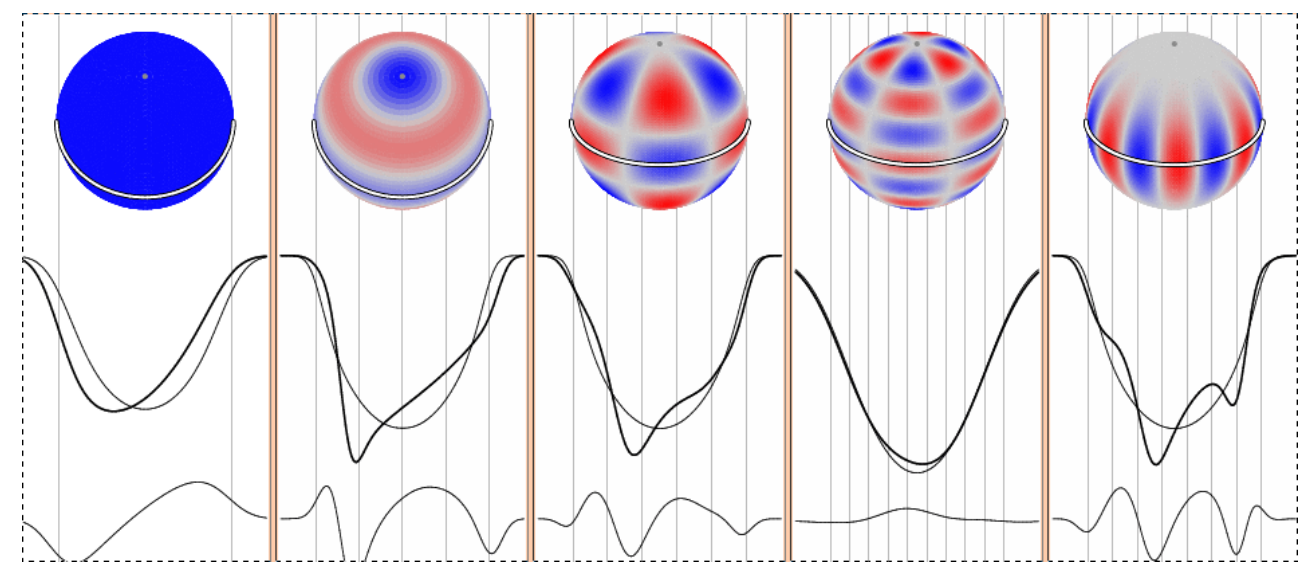

Figura 2.22. Aquí se muestra una "instantánea" de cómo son afectadas las líneas espectrales (líneas negras intermedias) por las pulsaciones. El primer objeto de la izquierda presenta una pulsación radial, mientras que el resto presentan pulsaciones no radiales. Las líneas negras inferiores corresponden a la diferencia entre la línea no afectada y la afectada por la pulsación, mientras que los colores azul y rojo hacen referencia a la dilatación y contracción local de la estrella, respectivamente. Extraído de http://staff.not.iac.es/ jht/science/

et al. 2016.

Por último, también hay que tener en cuenta la binaridad o multiplicidad, que también intervienen en la variabilidad de las BSGs, como puede apreciarse en la figura 2.23

\subsection{Mecanismos adicionales: pulsaciones ${ }^{21}$}

Hemos visto que las estrellas BSGs presentan variaciones en sus curvas de luz y estos cambios en el brillo pueden ser una manifestación de las pulsaciones estelares. Las pulsaciones son desplazamientos que sufre el gas de la estrella y dicho movimiento se realiza con una frecuencia en particular, produciendo cambios en el volumen de la estrella y, por lo tanto, cambios en sus propiedades físicas. Estos desplazamientos producen regiones de compresión y expansión del gas, lo que genera, a su vez, perturbaciones en la temperatura del gas, que en conjunto con variaciones en el radio, conducen a variaciones en el brillo de las estrellas, que es lo que observamos.

Existen dos tipos de pulsaciones: radiales y no-radiales. En una pulsación radial toda la estrella se contrae o se expante, manteniendo su forma esférica, pero en una pulsación noradial habrá regiones que se expanden y otras regiones que se contraen, simultáneamente. La característica física principal que diferencia a un tipo de pulsación de otro, es que en una pulsación radial la fuerza restauradora es la presión del gas, y en una pulsación no-radial la fuerza restauradora puede ser tanto la fuerza de presión como la fuerza de gravedad.

\footnotetext{
${ }^{21}$ Los conceptos básicos sobre las pulsaciones fueron sacados de los trabajos de tesis de Licenciatura de Monachesi y de la tesis de Doctorado de Córsico, disponibles en la página web del Grupo de Evolución Estelar de la FCAG https://fcaglp.fcaglp.unlp.edu.ar/evolgroup/
} 


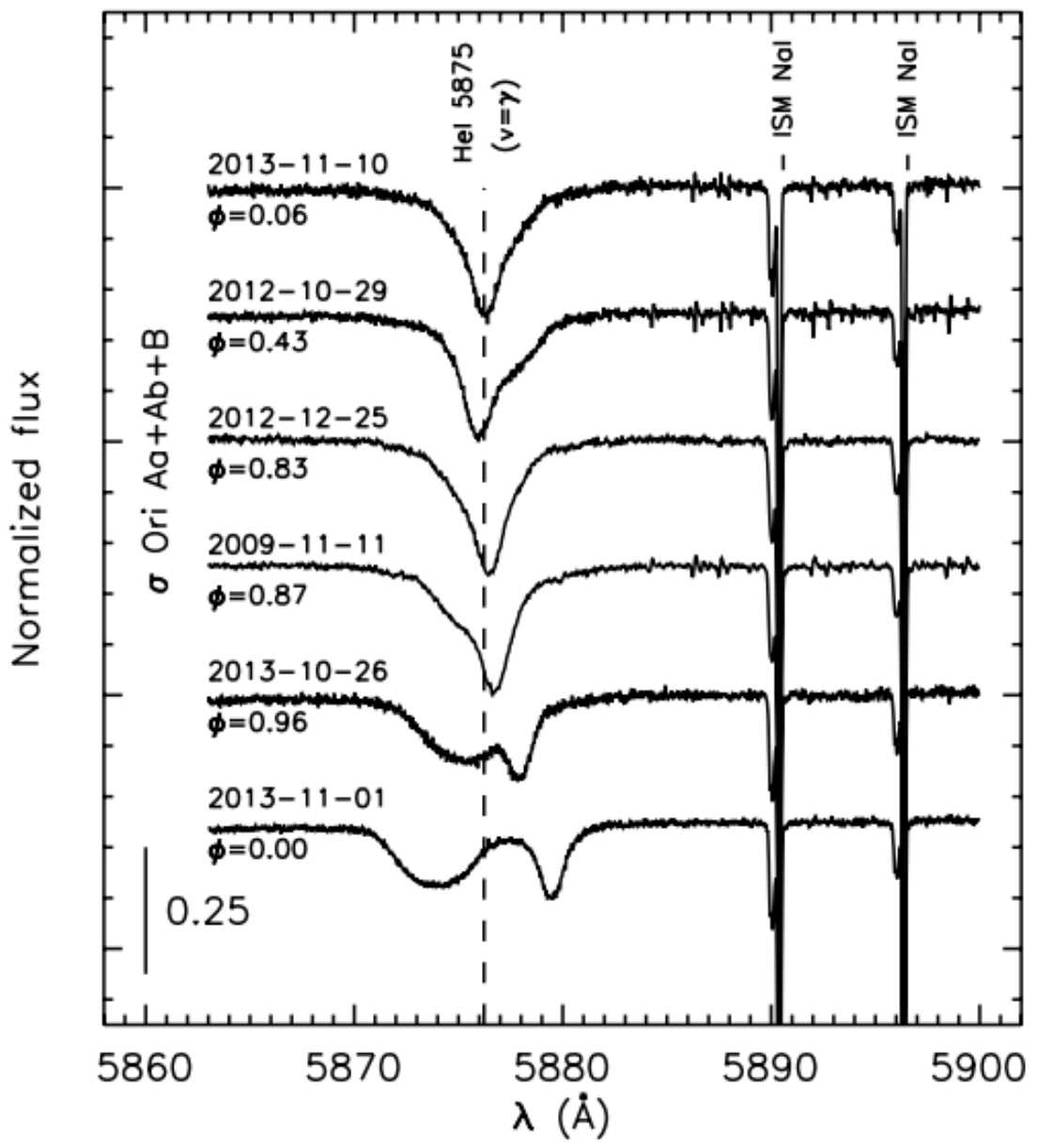

Figura 2.23. En este gráfico se muestra el sistema triple $\sigma$ Ori $\mathrm{Aa}+\mathrm{Ab}+\mathrm{B}$ y cómo va variando la línea de He I $\lambda 5875$ en forma e intensidad conforme a la fase orbital $(\phi)$. La línea vertical hace referencia a la longitud de onda del He I desplazada unos $31 \mathrm{~km} \mathrm{~s}^{-1}$ respecto a la longitud de onda en reposo (velocidad sistémica del sistema). Extraído de Simón-Díaz et al. 2015. 
Estos diferentes modos de pulsación se conocen como modos p y modos g, respectivamente. Los primeros se caracterizan por tener frecuencias cada vez más altas (períodos cortos), mientras que los segundos poseen frecuencias cada vez más bajas (períodos largos) a medida que el número de nodos (regiones donde no hay desplazamientos) aumenta. Las frecuencias naturales con la que una estrella puede oscilar se denominan autofrecuencias $(\sigma)$, ya que en un análisis pulsacional lineal lleva a resolver un problema de autovalores con dependencia temporal $\exp (\mathrm{i} \sigma \mathrm{t})$. La autofrecuencia $\sigma$ es un número complejo, es decir, $\sigma=\sigma_{\mathrm{r}}+\mathrm{i} \sigma_{\mathrm{i}}$, donde la parte real, $\sigma_{\mathrm{r}}$, da el período pulsacional $\left(2 \pi / \sigma_{\mathrm{r}}\right)$ y la parte imaginaria, $\sigma_{\mathrm{i}}$ da la estabilidad del modo de pulsación, es decir, si el modo es inestable $\sigma_{\mathrm{i}}<0$, y si el modo es estable $\sigma_{\mathrm{i}}>0$ Glatzel \& Kiriakidis 1993.

Los modos naturales de pulsación de una estrella tienen nodos en tres direcciones ortogonales: la distancia $r$ al centro de la estrella, la colatitud $\theta$ (medida a partir del polo de pulsación) y la longitud $\phi$ (medida a partir del ecuador de pulsación) y son necesarios tres números cuánticos para especificar los modos Aerts et al.2010a): el orden n, que está relacionado con el número radial de nodos; el grado l, que especifica el número de superficies nodales que están presentes; y el orden acimutal $m$, donde $|\mathrm{m}|(0<|\mathrm{m}|<\mathrm{l})$ especifica cuantas superficies nodales son líneas de longitud. La fig. 2.24 representa cómo se vería un modo no-radial con $\mathrm{l}=3$, a diferentes inclinaciones respecto al polo de pulsación y con diferentes valores para el número $m$. Desafortunadamente esta figura es sólo representativa ya que aún no están suficientemente desarrolladas las capacidades tecnológicas para resolver una superficie estelar y detectar directamente este fenómeno.

Como se mencionó, la pulsaciones son movimientos, y todo movimiento tiene asociada una energía, lo que conlleva a preguntarse cuál(es) es(son) el(los) mecanismo(s) que produce(n) una pulsación. El principal mecanismo está conectado con la opacidad, y se conoce como mecanismo $\kappa$. Para que este mecanismo funcione debe existir mucho material opaco a la radiación y los mayores desencadenantes de pulsaciones mediante este mecanismo son el hidrógeno y el helio. Las capas estelares internas ionizadas de $\mathrm{H}$ y He bloquean la radiación produciendo un calentamiento local del gas y un aumento de la presión y la temperatura, causando que la estrella deje de estar en equilibrio local. Pero el aumento de la temperatura reduce la opacidad, la radiación "escapa", el gas se enfría y debido al peso de las capas que están por encima, la estrella se contrae. En la contracción el H o el He se recombinan y el flujo es absorbido nuevamente. Se dice que una región que gana calor durante la compresión es una región de excitación y que si pierde calor en el mismo proceso, es una región de amortiguamiento y, por lo tanto, la inestabilidad o estabilidad de una estrella depende de cuál proceso se impone sobre el otro. Cabe mencionar que el mecanismo $\kappa$ también puede ser impulsado no sólo por el $\mathrm{H}$ y el He, sino también por el pico en las opacidades de los elementos del grupo del hierrd22. Existen otros mecanismos, como el mecanismo $\gamma$, el mecanismo $\epsilon$ o el mecanismo estocástico (ver detalles en Aerts et al.2010a , pero que en principio no estarían presentes en las estrellas BSGs.

Si bien las microvariaciones observadas en las curvas de luz de las variables $\alpha$ Cyg no son nuevas Burki et al. 1978, van Genderen 1989, entre otros), el mecanismo que las produce

\footnotetext{
22 Le Pennec \& Turck-Chièze 2014, Fe I, Fe II y Fe III)
} 


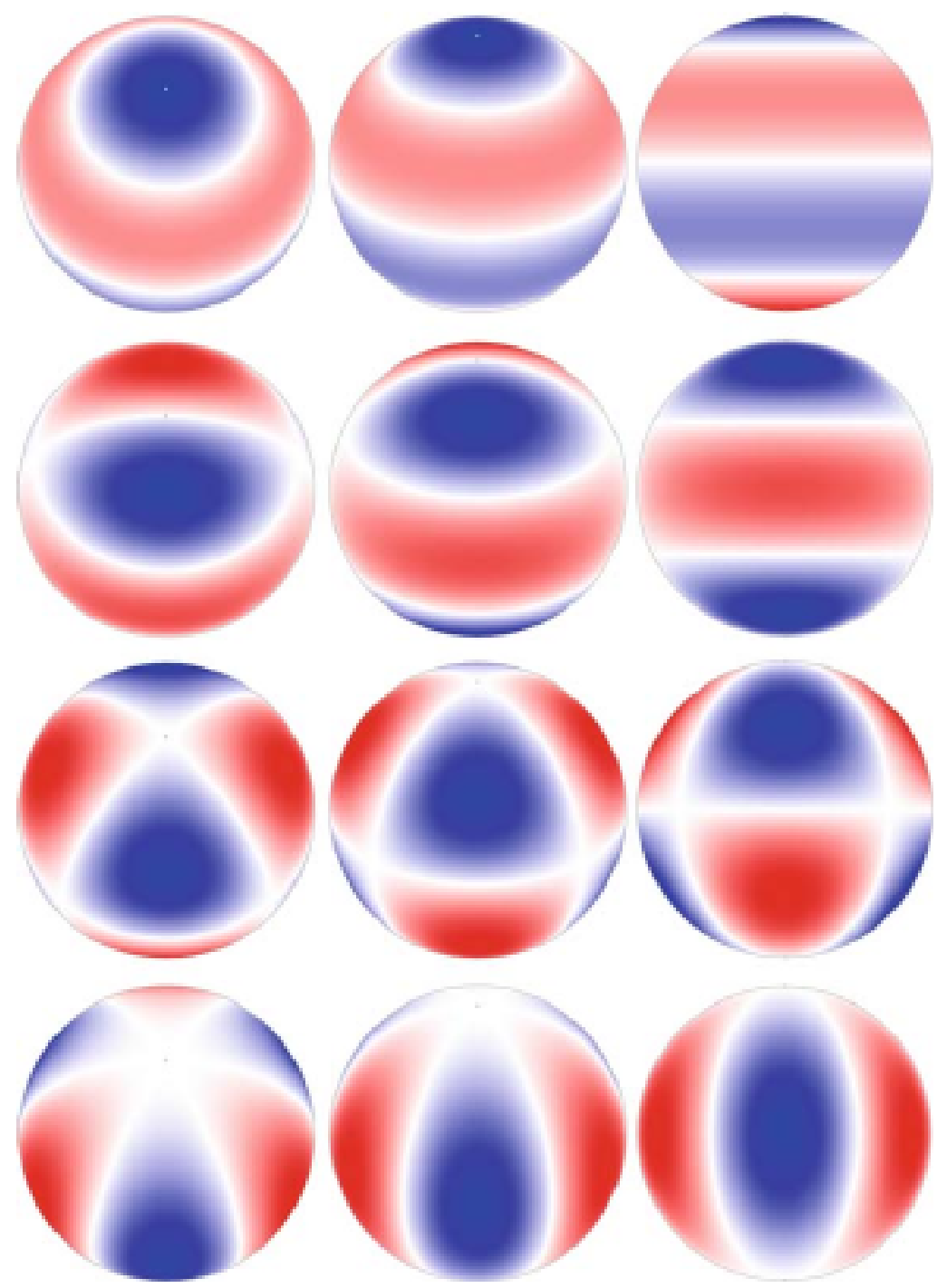

Figura 2.24. Modo no-radial con $l=3$. Las regiones azules y rojas corresponden a regiones de expansión y contracción respectivamente, mientras que las líneas blancas representan las posiciones de las superficies nodales. Cada columna está caracterizada por un ángulo de inclinación del polo de pulsación: la primera tiene un ángulo de $30^{\circ}$, la segunda de $60^{\circ}$ y la tercera de $90^{\circ}$. La primera fila tiene el par de números cuánticos $(\mathrm{l}=3, \mathrm{~m}=0)$ y tres nodos en $\pm 51^{\circ}$ y $0^{\circ}$; la segunda fila representa un modo con $(1=3, \mathrm{~m}= \pm 1)$ con dos nodos que son líneas de latitud y un nodo que es una línea de longitud; la tercera fila tiene el conjunto de números cuánticos $(\mathrm{l}=3, \mathrm{~m}= \pm 2)$; y la última fila $(\mathrm{l}=3, \mathrm{~m}= \pm 3)$. El signo de $m$ es importante si se tiene en cuenta la rotación de la estrella. Extraído de Aerts et al. 2010a. 
aún no está del todo claro. Mediante el análisis de curvas de luz tomadas por el satélite HIPPARCOS, Waelkens et al. 1998) y Lefever et al. 2007) propusieron al mecanismo $\kappa$ como el responsable de las variaciones de luz. En ambos trabajos también se menciona la posible presencia de inestabilidades de modos extraños como mecanismo adicional y coexistente al mecanismo $\kappa$. Shibahashi \& Osaki 1981 sugirieron que la existencia de estos modos extraños en las oscilaciones no radiales de modelos estelares de alta luminosidad $\left(\mathrm{L}=10^{5} \mathrm{~L}_{\odot}\right)$ podrían ser causados por fuertes efectos no adiabáticos acoplados con inestabilidades convectivas. Por otro lado, Glatzel \& Kiriakidis (1993) y Glatzel 1994) argumentaron que la causa de estos modos extraños tiene un origen acústico ya que sus frecuencias están relacionadas con el tiempo de viaje del sonido entre la fotosfera estelar y la posición del máximo de opacidad. En otras palabras, si el gradiente de velocidad del sonido es grande, las ondas sonoras son reflejadas, es decir, que "saltos" en la velocidad del sonido podrían servir como barreras que acústicamente desacoplen diferentes partes de la estrella. Matemáticamente, lo que sucede cuando se resuelve un sistema de ecuaciones para oscilaciones no-radiales de modelos estelares de alta luminosidad $\left(\mathrm{L}=10^{5} \mathrm{~L}_{\odot}\right)$, es que aparece un nuevo tipo de valor propio, para el cual la parte imaginaria de la autofrecuencia es tan grande como la parte real y no tiene una contrapartida adiabática, mientas que para el caso de modelos con $\mathrm{L}<10^{4} \mathrm{~L}_{\odot}$, la parte imaginaria de la autofrecuencia es siempre mucho más chica que la parte real, y que el valor de esta última toma un valor muy cercano al correspondiente a las oscilaciones adiabáticas (Shibahashi \& Osaki 1981). Saio 2009 concluyó que los modos extraños radiales aparecen cuando la energía de pulsación está fuertemente confinada en una región donde la presión de radiación es mucho mayor que la presión del gas, y que la inestabilidad de los modos extraños ocurren en regiones extremadamente no adiabáticas.

Interpretar las observaciones para comprender los posibles modos de oscilación y cómo es la dependencia de sus frecuencias con las propiedades del interior estelar es el principal objetivo de la astrosismología. A partir de cómputos numéricos de oscilaciones para diferentes modelos estelares, es posible determinar las regiones del diagrama HR donde los diferentes modos pueden estar excitados. Esto puede verse en la fig. 2.25, que representa las diferentes regiones (puntos rojos) donde los modos radiales estarían excitados Saio et al. 2013a. Estas regiones de inestabilidad de pulsaciones radiales por el mecanismo $\kappa$ presentan gradientes bien marcados en el diagrama HR, como por ejemplo en las dos regiones con $\log \mathrm{L} / \mathrm{L}_{\odot} \leq 5$, donde las líneas a rayas y punteadas representan a la región con $\mathrm{Z}=$ 0.014 y Z $=0.02$, respectivamente. A altas luminosidades, la región de inestabilidad es casi horizontal y está asociada con los modos extraños. En este mismo estudio de pulsaciones radiales y no-radiales en estrellas BSGs, Saio et al. 2013a llegan a la conclusión de que la mayoría de las variables $\alpha$ Cyg poseen variaciones cuasiperiódicas, lo que indica que muchos períodos están simultáneamente excitados. Encontraron también que la mayoría de estas variables (círculos y cuadrados azules, para estrellas BSGs de la Vía Láctea y NGC 300, respectivamente, en la fig. 2.25 se encuentran entre $3.8 \leq \log \left(\mathrm{T}_{\text {ef }}\right) \leq 4.3$ y donde los modelos predicen la excitación de las pulsaciones radiales sólo si la estrella pasó previamente por la fase de supergigante roja, es decir, que serían objetos que se encuentran por segunda vez en la fase de BSG. Por otro lado, estos autores también encuentran que 


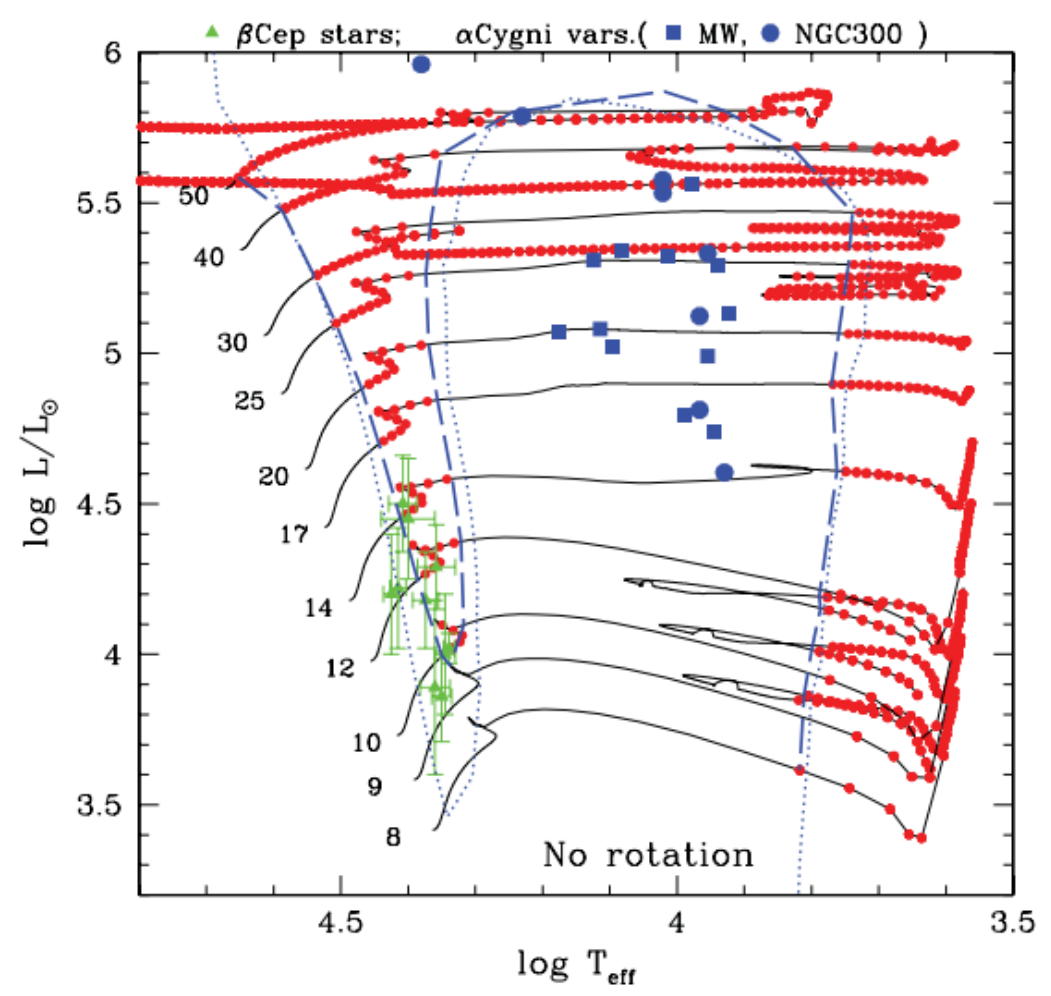

Figura 2.25. Diagrama HR donde se muestran las regiones donde se excita al menos un modo radial (puntos rojos) en conjunto con los tracks evolutivos para diferentes masas iniciales (líneas continuas negras), sin rotación y metalicidad solar. Los puntos y cuadrados azules corresponden a variables $\alpha$ Cygni y los verdes a variables $\beta$ Cep. Extraído de Saio et al. 2013a.

hay una mayor cantidad de modos de pulsación no-radiales excitados antes de la fase de RSG que después de esta fase evolutiva.

En este capítulo hemos presentado una revisión de cómo ha ido evolucionando la teoría de los vientos estelares, mostrando los acuerdos y desacuerdos que surgen con las observaciones. También destacamos la importancia de la tasa de pérdida de masa y la composición química en la evolución estelar, y el posible papel que tendrían las pulsaciones en la variabilidad observada en las estrellas BSGs. Por lo tanto, todo lo descripto nos brinda un marco para introducir el trabajo que hemos realizado. Con el objetivo de contribuir al entendimiento de este tipo de estrellas, estudiamos una muestra de 20 estrellas BSGs a partir de datos espectroscópicos de alta resolución y los resultados que hemos obtenido se mostrarán en los siguientes capítulos. 


\section{Capítulo 3}

\section{Análisis de una muestra de supergigantes $\mathrm{B}^{\prod}$}

\subsection{Resumen}

Este capítulo está dedicado a la determinación de los parámetros estelares y del viento de una muestra de 19 estrellas BSGs galáctica $\bigsqcup^{2}$. La técnica usada para determinar las propiedades de las estrellas y sus vientos está basada en la síntesis espectral y los valores obtenidos nos permitirán discutir en el cap. 5 cómo se comportan estos objetos y poder comparar nuestros resultados con los de otros autores.

En la primera sección de este capítulo describiremos la técnica de adquisición de los datos espectroscópicos. En las seccs. 3.3 y 3.4 presentaremos los parámetros estelares y del viento, respectivamente, junto con las estimaciones de los errores de cada uno de ellos. Además en la secc. 3.5 se mostrarán los resultados de los ajustes realizados para cada estrella, junto con comentarios individuales de cada objeto.

\subsection{Observaciones}

Adquirimos espectros de alta resolución de 19 estrellas BSG galácticas de tipos espectrales entre B0 y B9, seleccionadas del Bright Star Catalog (Hoffleit \& Jaschek 1991). Las observaciones corresponden a diferentes épocas: enero de 2006, febrero de 2013, abril de 2014, y febrero y marzo de 2015. Para la adquisición de los datos se utilizó el espectrógrafo REOSC, en su modo de dispersión cruzada, anexado al telescopio Jorge Sahade 2.15 m del

\footnotetext{
${ }^{1}$ Basada en observaciones tomadas con el Telescopio J. Sahade del Complejo Astronómico El Leoncito (CASLEO), operado bajo el acuerdo entre el Consejo Nacional de Investigaciones Científicas y Técnicas de la República Argentina, la Secretaría de Ciencia y Tecnología de la Nación y las Universidades Nacionales de La Plata, Córdoba y San Juan.

${ }^{2}$ Los resultados fueron publicados en la revista Astronomy \& Astrophysics, titulado Wind Properties of Variable B Supergiants: Evidence of Pulsations Connected with Mass-loss Episodes, por Haucke et al. (2018) http://adsabs. harvard. edu/abs/2018A\%26A...614A..91H
} 
Complejo Astronómico El Leoncito (CASLEO), San Juan, Argentina. Se usó la red de 400 $\mathrm{l} / \mathrm{mm}$, una ranura simple de $250 \mu$ de ancho y el CCD TEK de $1024 \times 1024$ con una ganancia de $1.98 \mathrm{e}^{-}$/adu. Esta configuración nos brindó una resolución $\mathrm{R} \sim 12600$ en $6500 \AA$ A. Los espectros, reducidos mediante los paquetes tradicionales de IRAF, tienen una relación $\mathrm{S} / \mathrm{N}$ $\sim 300$.

En la tabla 3.1 se listan las estrellas BSGs estudiadas, indicando su nombre y número $\mathrm{HD}$, tipo espectral, tipo de variable designada de acuerdo al trabajo de Lefèvre et al. 2009, a partir de curvas de luz de HIPPARCOS) o de la base de datos VSX ${ }^{3}$ En las columnas subsiguientes se listan los períodos fotométricos o espectroscópicos reportados en la literatura, las épocas de observación y el rango espectral cubierto. En esta muestra hay seis estrellas con más de una observación: HD 53138, HD 58350, HD 75149, HD 80077, HD 99953 y HD 111973 (en particular esta última tiene dos observaciones en dos noches consecutivas).

\subsection{Determinación de parámetros estelares}

Para derivar los parámetros estelares y del viento usamos el código FASTWIND, en su versión 10.1.7. Como se mencionó en la secc. 2.7.1. este código resuelve el transporte radiativo de una atmósfera en expansión con simetría esférica libre de campo magnético. En este estudio hemos adoptado los valores para la abundancia solar reportados por Grevesse \& Sauval 1998 para todos los elementos.

Para obtener la temperatura efectiva $\left(\mathrm{T}_{\mathrm{ef}}\right)$ evaluamos el balance de ionización entre Si II y Si III, Si III y Si IV (en caso de contar con las líneas Si IV $\lambda 4089 \AA$ y $\lambda 4116 \AA$ ) y He I y He II. Para derivar de manera precisa la gravedad superficial (log g) modelamos las líneas $\mathrm{H}_{\gamma}$ ( $\lambda 4341 \AA)$ y $\mathrm{H}_{\delta}(\lambda 4102 \AA)$, cuando estas están disponibles en el rango espectral observado, ya que son las líneas de Balmer clásicas para determinar este parámetro Markova \& Puls 2008). Los perfiles teóricos y observados fueron ajustados por inspección visual.

Previamente al proceso de modelado de los perfiles de línea, el radio estelar $\mathrm{R}_{\star}$ fue derivado usando varios de los métodos descriptos en la secc. 2.4. en particular a través del ajuste de la SED, el diámetro angular y la distancia. Para el primer método se utilizó la interface interactiva BeSOS, que, a partir de un exceso de color dado, provee un nuevo conjunto de parámetros estelares: $\mathrm{T}_{\text {ef }}, \log \mathrm{g}$ y $\mathrm{R}_{\star}$ (en unidades de radios solares). Los valores iniciales de entrada que utilizamos para ajustar la SED son $\mathrm{T}_{\text {ef }} \mathrm{y} \log \mathrm{g}$ que obtuvimos en este trabajo a partir del balance de ionización de Si y He, y de los anchos equivalentes de las líneas $\mathrm{H}_{\gamma} \mathrm{y} \mathrm{H}_{\delta}$. Luego el código ajusta la $\mathrm{SED}$ para los parámetros más óptimos (ver fig. 3.1. El exceso de color, E(B-V), se calculó mediante el B-V observado y el $(B-V)_{0}$ interpolado a partir de la escala $\mathrm{T}_{\mathrm{ef}}-(\mathrm{B}-\mathrm{V})$ para supergigantes Flower 1996. Por último, el radio estelar determinado con este ajuste fue promediado con los valores obtenidos por otras técnicas, ya sea empleando el valor medio del tamaño angular o la magnitud bolométrica.

\footnotetext{
${ }^{3}$ The International Variable Star Index, https://www.aavso.org/vsx/
} 
Tabla 3.1. Registro de las observaciones.

\begin{tabular}{|c|c|c|c|c|c|c|}
\hline Nombre & HD & $\mathrm{TE}^{a}$ & Tipo de var. ${ }^{b}$ & $\begin{array}{l}\text { Período } \\
\text { (días) }\end{array}$ & $\begin{array}{l}\text { Época de obs. } \\
\text { (AAAA/MM/DD) }\end{array}$ & $\begin{array}{c}\text { Rango espectral } \\
{[\AA: \AA]}\end{array}$ \\
\hline$\beta$ Ori & 34085 & B8Iae & $\mathrm{ACYG}$ & $2.075^{c}, 1.22-74.74^{d}, 28^{n}$ & $2006 / 01 / 15$ & {$[3500: 7850]$} \\
\hline$\kappa$ Ori & 38771 & B0.5Ia & IA & $1.047^{e}, 1.9^{f}, 4.76^{e}, 6.5^{f}, 9.5^{f}$ & $2006 / 01 / 15$ & [3500:7850] \\
\hline$\chi^{2}$ Ori & 41117 & $\mathrm{~B} 2 \mathrm{Ia}$ & $\mathrm{ACYG}$ & $0.92^{e}, 0.95^{e}, 2.869^{e}, 20^{e}, 40^{e}, 200^{e}$ & $2006 / 01 / 15$ & [3500:7850] \\
\hline PU Gem & 42087 & $\mathrm{~B} 4 \mathrm{Ia}^{g}$ & $\mathrm{ACYG}$ & $6.807^{\circ}, 25^{e}$ & $2006 / 01 / 15$ & [6500:7850] \\
\hline V731 Mon & 47240 & $\mathrm{~B} 1 \mathrm{Ib}$ & $\mathrm{ACYG}, \mathrm{SB}^{q}$ & $1.73^{j}, 2.742^{c, e}, 133^{e}$ & $2006 / 01 / 15$ & [3500:7850] \\
\hline$\epsilon \mathrm{CMa}$ & 52089 & B1.5 II & IA & & $2013 / 02 / 05$ & [4300:6850] \\
\hline V820 Cas & 52382 & B1Ia & IA & & $2006 / 01 / 15$ & [3500:7850] \\
\hline$\sigma^{2} \mathrm{CMa}$ & 53138 & B3Ia & $\mathrm{ACYG}$ & $3.69^{j}, 24.39^{j}, 24.44^{c}$ & $\begin{array}{l}2006 / 01 / 15 \\
2013 / 02 / 05\end{array}$ & $\begin{array}{l}{[3500: 7850]} \\
{[4300: 6850]}\end{array}$ \\
\hline$\eta \mathrm{CMa}$ & 58350 & B5Ia & $\mathrm{L}, \mathrm{ACYG}$ & $4.7^{i}, 6.631^{j}$ & $\begin{array}{l}2006 / 01 / 15 \\
2013 / 02 / 05\end{array}$ & [3500:7850] \\
\hline J Pup & 64760 & $\mathrm{~B} 0.5 \mathrm{Ib}$ & IA & $1.2^{m}, 1.8^{j}, 2.4^{m}, 2.8^{j}, 6.8^{m}$ & $2013 / 02 / 05$ & [4300:6850] \\
\hline LN Vel & 74371 & $\mathrm{~B} 6 \mathrm{Iab} / \mathrm{b}$ & $\mathrm{IA}, \mathrm{ACYG}$ & $8.29^{c, i}, 1,15-20^{h}$ & $2006 / 01 / 15$ & {$[3500: 7850]$} \\
\hline OP Vel & 75149 & B3Ia & SPB?, ACYG & $1.086^{c, n}, 1.215^{j}, 2.214^{j}$ & $\begin{array}{l}2006 / 01 / 15 \\
2013 / 02 / 05 \\
2013 / 02 / 07 \\
2014 / 04 / 14\end{array}$ & $\begin{array}{l}{[3500: 7850]} \\
{[4300: 6850]} \\
{[4300: 6850]} \\
{[4200: 6650]}\end{array}$ \\
\hline GX Vel & 79186 & B5Ia & IA & & $2006 / 01 / 15$ & {$[3500: 7850]$} \\
\hline PV Vel & 80077 & $\mathrm{~B} 2 \mathrm{Ia}+\mathrm{e}$ & GCAS?, SDOR & $3.115^{c}, 21.2^{l}, 41.5^{h}, 55.5^{h}, 66.5^{h}, 76.0^{h}$ & $\begin{array}{l}2006 / 01 / 15 \\
2014 / 04 / 12\end{array}$ & $\begin{array}{l}{[3500: 7850]} \\
{[4200: 6650]}\end{array}$ \\
\hline V519 Car & 92964 & $\mathrm{~B} 2.5 \mathrm{Ia}$ & $\mathrm{ACYG}$ & $2.119^{j}, 4.71^{c}, 14.706^{j}$ & $2013 / 02 / 05$ & {$[4300: 6850]$} \\
\hline V808 Cen & 99953 & $\mathrm{~B} 1 / 2 \mathrm{Iab} / \mathrm{b}$ & IA & $17.7^{k}$ & $\begin{array}{l}2014 / 04 / 14 \\
2015 / 02 / 13 \\
2015 / 03 / 13\end{array}$ & $\begin{array}{l}{[4200: 6650]} \\
{[4200: 6650]} \\
{[4200: 6650]}\end{array}$ \\
\hline$\kappa \mathrm{Cru}$ & 111973 & $\mathrm{~B} 2 / 3 \mathrm{Ia}$ & $\mathrm{ACYG} ?, \mathrm{IA}, \mathrm{SB}^{p}$ & $9.536^{i}, 57.11^{i}$ & $\begin{array}{l}2014 / 04 / 11 \\
2014 / 04 / 12\end{array}$ & $\begin{array}{l}{[4200: 6650]} \\
{[4200: 6650]}\end{array}$ \\
\hline ALS 3038 & 115842 & $\mathrm{~B} 0.5 \mathrm{Ia} / \mathrm{ab}$ & ACYG?, IA & $10.309^{c}, 13.38^{i}$ & $2014 / 04 / 11$ & {$[4200: 6650]$} \\
\hline V1058 Sco & 148688 & B1Iaeqp & $\mathrm{ACYG}$ & $1.845^{c}, 6.329^{c}$ & $2014 / 04 / 11$ & {$[4200: 6650]$} \\
\hline
\end{tabular}

${ }^{a}$ De la base de datos astronómica SIMBAD Wenger et al. 2000 ${ }^{b} \mathrm{IA}$ (variables poco estudiadas de tipo espectral temprano), L (variables irregulares lentas), GCAS (variables del tipo $\gamma$ Cassiopea), ACYG (variables del tipo $\alpha$ Cyg ), SPB (estrellas B de pulsación lenta), y S Dor, variables del tipo S Doradus (clasificación tomada de Lefèvre et al. 2009). El signo de interrogación significa que la clasificación fue basada en una nueva determinación de período o que el tipo de variable no pudo ser indentificado claramente a partir del análisis de las curvas de

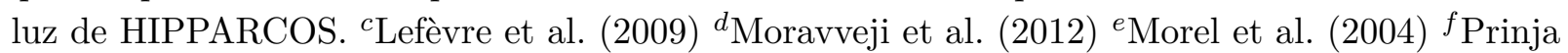

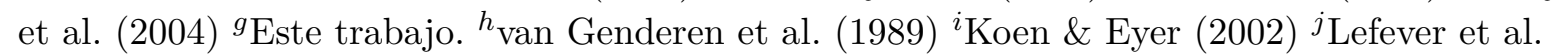
2007 SSterken 1977) Knoechel \& Moffat (1982 m Kaufer et al. (2006) "Aerts et al. 2013)

${ }^{o}$ Base de datos del Indice Internacional de Estrella Variable (VSX), y ${ }^{p, q} \mathrm{SB}$ (binaria espectroscópica Chini et al. 2012 Prinja et al. 2002. 

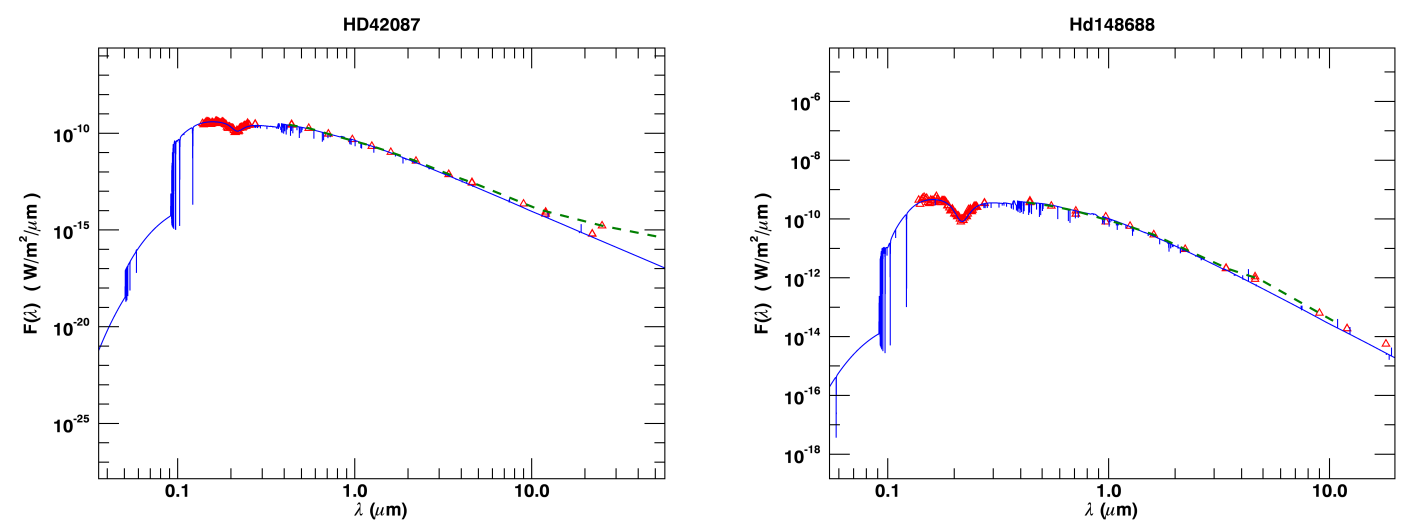

Figura 3.1. Ajuste de la distribución espectral de energía de HD 42087 (izquierda) y HD 148688 (derecha). Los triángulos rojos corresponden a los flujos observados y la línea continua azul corresponde al modelo.

La tabla 3.2 muestra para cada estrella sus propiedades intrínsecas: el tipo espectral encontrado en la literatura, la magnitud visual aparente, los excesos de colores observado e intrínseco, los parámetros estelares ( $\mathrm{T}_{\text {ef }} \mathrm{y} \log \mathrm{g}$ ) obtenidos con el ajuste de la SED, la distancia (d), la corrección bolométrica (CB), la magnitud bolométrica calculada, y el radio.

Los errores derivados para $T_{\text {ef }}, \log$ g y $R_{\star}$ usando BeSOS son aproximadamente del $1 \%$, $2 \%$ y $5 \%$, respectivamente Arcos et al. 2017). Sin embargo, considerando incertezas en la estimación de la distancia entre el $5 \%$ y el $30 \%$ (a partir de mediciones de paralaje) y diferencias menores a $1500 \mathrm{~K}$ entre los valores de $\mathrm{T}_{\text {ef }}$ derivados empleando los códigos BeSOS y FASTWIND, la propagación de errores arroja una incerteza del $10 \%$ para $\mathrm{M}_{\text {bol }}$ y un margen de error de entre el $5 \%$ y $20 \%$ para $R_{\star}$.

En relación a los errores de los parámetros estelares estimados con FASTWIND, adoptamos $\Delta \mathrm{T}_{\mathrm{ef}}=1000 \mathrm{~K}$ para las estrellas donde sólo contamos con el balance de ionización del He y $\Delta \mathrm{T}_{\text {ef }}=500 \mathrm{~K}$ para las estrellas con estimaciones de temperatura empleando las líneas de SiII, SiIII y He. El error en log g, que estimamos a partir de mediciones de las alas de las líneas $\mathrm{H}_{\gamma} \mathrm{y} \mathrm{H}_{\delta}$, es de 0.1 dex. Las barras de error en $\mathrm{v}_{\text {mic }}$ son de $2 \mathrm{~km} / \mathrm{s}$ para las líneas de $\mathrm{Si}$ y He, $5 \mathrm{~km} / \mathrm{s}$ para la líneas de $\mathrm{H}$ (excepto $\mathrm{H}_{\alpha}$ ) y $10 \mathrm{~km} / \mathrm{s}$ para $\mathrm{H}_{\alpha}$. Hemos modelado todas las líneas fotosféricas usando la misma $v_{\text {mic }}$ en cada estrella.

Adicionalmente, para obtener buenos ajustes a los perfiles observados, fue necesario considerar no sólo la velocidad de microturbulencia $\left(\mathrm{v}_{\mathrm{mic}}\right)$ y el ensanchamiento debido a la rotación de la estrella ( $\mathrm{v} \sin \mathrm{i}$ ), sino que también se tuvo que tener en cuenta la velocidad de macroturbulencia ( $\mathrm{v}_{\mathrm{mac}}$ ), que cambia tanto la intensidad de los perfiles como su forma Simón-Díaz \& Herrero 2007a. Los valores de v sin i y v mac afectan al ensanchamiento del perfil de la misma forma, y para modelar las líneas hemos adoptado los valores de vin i encontrados en la literatura Kudritzki et al. 1999 Lefever et al. 2007 Fraser et al. 2010. dados en la tabla 6.1). Estos valores fueron modificados en algunos casos considerando 
Tabla 3.2. Parámetros estelares calculados en este trabajo o adoptados de la literatura

\begin{tabular}{|c|c|c|c|c|c|c|c|c|c|c|c|}
\hline$\overline{\mathrm{HD}}$ & $\overline{\mathrm{TE}}$ & $m_{\mathrm{v}}$ & $\bar{B} B-V$ & $\overline{(B-V)_{0}{ }^{a}}$ & $\overline{E E(B-V)}$ & $\begin{array}{c}T_{\text {ef }} \\
\mathrm{K} \\
\end{array}$ & $\begin{array}{c}\log g \\
\operatorname{dex} \\
\end{array}$ & $\begin{array}{c}d \\
\mathrm{pc} \\
\end{array}$ & $\overline{c B C^{a}}$ & 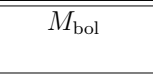 & $\begin{array}{l}R_{\star} \\
\mathrm{R}_{\odot} \\
\end{array}$ \\
\hline 34085 & B8 Iae & 0,13 & $-0,03$ & $-0,07$ & 0,04 & $11760 \pm 120$ & $2,00 \pm 0,10$ & $264 \pm 23$ & $-0,83$ & $-7,9 \pm 0,2$ & $72 \pm 10$ \\
\hline 38771 & B0.5 Ia & 2,06 & $-0,18$ & $-0,23$ & 0,05 & $25700 \pm 260$ & $2,70 \pm 0,05$ & $198 \pm 8$ & $-2,41$ & $-7,0 \pm 0,2$ & $13 \pm 1$ \\
\hline 41117 & B2 Ia & 4,63 & 0,28 & $-0,18$ & 0,42 & $17940 \pm 180$ & $2,20 \pm 0,05$ & $552 \pm 85$ & $-1,84$ & $-7,3 \pm 0,4$ & $23 \pm 3$ \\
\hline 42087 & B4 Ia & 5,78 & 0,20 & $-0,14$ & 0,34 & $15000 \pm 150$ & $2,39 \pm 0,05$ & $2075 \pm 1000^{s}$ & $-1,46$ & $-8,3 \pm 1,1$ & $55 \pm 11$ \\
\hline 47240 & B1 Ib & 6,15 & 0,15 & $-0,18$ & 0,33 & $17500 \pm 180$ & $2,40 \pm 0,05$ & $1598 \pm 479$ & $-1,78$ & $-7,7 \pm 0,7$ & $30 \pm 4$ \\
\hline 52089 & B1.5 II & 1,50 & $-0,21$ & $-0,20$ & 0,00 & $21000 \pm 210$ & $3,00 \pm 0,05$ & $124 \pm 2$ & $-2,20$ & $-6,2 \pm 0,1$ & $11 \pm 1$ \\
\hline 52382 & B1 Ia & 6,50 & 0,19 & $-0,20$ & 0,44 & $23140 \pm 230$ & $2,47 \pm 0,05$ & $1301 \pm 430^{s}$ & $-2,05$ & $-7,5 \pm 0,8$ & $21 \pm 2$ \\
\hline 53138 & B3 Ia & 3,02 & $-0,08$ & $-0,21$ & 0,10 & $18000 \pm 180$ & $2,20 \pm 0,05$ & $847 \pm 287$ & $-1,66$ & $-8,5 \pm 0,8$ & $46 \pm 4$ \\
\hline 58350 & B5 Ia & 2,45 & $-0,09$ & $-0,17$ & 0,08 & $15000 \pm 500^{f}$ & $2,00 \pm 0,10^{f}$ & $609 \pm 148$ & $-1,24$ & $-8,0 \pm 0,6$ & $54 \pm 6$ \\
\hline 64760 & B0.5 Ib & 4,24 & $-0,14$ & $-0,21$ & 0,07 & $22370 \pm 220$ & $2,50 \pm 0,05$ & $507 \pm 25$ & $-2,21$ & $-6,7 \pm 0,2$ & $12 \pm 1$ \\
\hline 74371 & B6 Iab/b & 5,23 & 0,20 & $-0,15$ & 0,35 & $13800 \pm 140$ & $2,00 \pm 0,05$ & $1800 \pm 360^{s}$ & $-1,02$ & $-8,7 \pm 0,5$ & $73 \pm 10$ \\
\hline 75149 & B3 Ia & 5,46 & 0,27 & $-0,19$ & 0,46 & $15000 \pm 150$ & $2,12 \pm 0,05$ & $1642 \pm 330^{s}$ & $-1,39$ & $-8,4 \pm 0,7$ & $61 \pm 13$ \\
\hline 79186 & B5 Ia & 5,00 & 0,22 & $-0,13$ & 0,35 & $15000 \pm 150$ & $2,12 \pm 0,05$ & $1449 \pm 609$ & $-1,36$ & $-8,3 \pm 0,9$ & $61 \pm 7$ \\
\hline 80077 & $\mathrm{~B} 2 \mathrm{Ia}+\mathrm{e}$ & 7,56 & 1,34 & $-0,16$ & 1,50 & $18000 \pm 180$ & $2,17 \pm 0,05$ & $3600 \pm 600^{s}$ & $-1,62$ & $-11,6 \pm 0,4$ & $195 \pm 47$ \\
\hline 92964 & B2.5Ia & 5,38 & 0,27 & $-0,16$ & 0,48 & $18000 \pm 180$ & $2,19 \pm 0,05$ & $1851 \pm 994$ & $-1,66$ & $-9,1 \pm 1,2$ & $70 \pm 14$ \\
\hline 99953 & $\mathrm{~B} 1 / 2 \mathrm{Iab} / \mathrm{b}$ & 6,5 & 0,38 & $-0,18$ & 0,56 & $18830 \pm 190$ & $2,30 \pm 0,05$ & $1075 \pm 427$ & $-1,78$ & $-7,2 \pm 0,9$ & $25 \pm 3$ \\
\hline 111973 & $\mathrm{~B} 2 / 3 \mathrm{Ia}$ & 5,94 & 0,24 & $-0,14$ & 0,38 & $17180 \pm 170$ & $2,18 \pm 0,05$ & $1660 \pm 350^{s}$ & $-1,46$ & $-7,8 \pm 0,5$ & $46 \pm 9$ \\
\hline 115842 & B0.5 Ia/ab & 6,03 & 0,30 & $-0,23$ & 0,60 & $25830 \pm 260$ & $2,75 \pm 0,05$ & $1538 \pm 750$ & $-2,46$ & $-9,2 \pm 1,2$ & $35 \pm 4$ \\
\hline 148688 & B1 Iaeqp & 5,31 & 0,35 & $-0,20$ & 0,54 & $20650 \pm 210$ & $2,20 \pm 0,05$ & $833 \pm 229$ & $-2,00$ & $-8,0 \pm 0,7$ & $31 \pm 4$ \\
\hline
\end{tabular}

${ }^{a} \mathrm{CB}$ Flower 1996, ${ }^{f}$ parámetro derivado con FASTWIND y ${ }^{s}$ distancias derivadas por diferentes métodos (ver detalles en el texto).

variaciones de $\pm 10 \mathrm{~km} / \mathrm{s}$ para tener un mejor ajuste. La velocidad de macroturbulencia fue derivada del modelado al tratar de reproducir el ensanchamiento extra presente en las líneas. Hemos encontrado incertezas entre $10 \mathrm{~km} / \mathrm{s}$ y $20 \mathrm{~km} / \mathrm{s}$ para $\mathrm{v}_{\mathrm{mac}}$.

\subsection{Parámetros del viento}

La línea $\mathrm{H}_{\alpha}$ fue modelada para derivar la tasa de pérdida de masa $(\dot{\mathrm{M}})$ y los parámetros del campo de velocidades del viento: el índice $\beta$ y la velocidad terminal $\left(\mathrm{v}_{\infty}\right)$. Pero antes de involucrarnos con los parámetros del viento es importante remarcar que la mayor incerteza en la determinación de $\dot{M}$ es debido a las ambigüedades en la determinación del radio estelar y la distancia al objeto. Markova et al. 2004 encuentran importantes discrepancias entre las tasas de pérdida de masa derivadas por ellos respecto a otros autores para el mismo objeto, y atribuyen dichas discrepancias al hecho de que las determinaciones de las distancias a los objetos (sobre todo galácticos) son muy inciertas. La dificultad de tener valores precisos de $d$ causan muchos problemas a la hora de determinar $\mathrm{R}_{\star} \mathrm{y}$ los parámetros del viento de las estrellas galácticas (Markova et al. 2004). Por otro lado, puesto que $\mathrm{H}_{\alpha}$ es más bien sensible a $\mathrm{v}_{\infty}$ Garcia et al.2017, los valores para el radio y la velocidad terminal son derivados de manera independiente.

Para determinar los parámetros del viento utilizamos modelos de atmósferas sin clumping por dos motivos: el primero surge de la necesidad de comparar los valores de las tasas de pérdida de masa que hemos medido con los determinados por otros autores y estudiar así su variabilidad. Por lo general, la mayoría de los valores encontrados en la literatu- 
ra fueron determinados a partir de modelos sin clumping. La segunda razón es que para lograr un tratamiento incorporando este factor, es necesario contar con observaciones en otros rangos espectrales como lo indican los trabajos de Repolust et al. 2005 y Puls et al. 2006). Como consecuencia de ignorar los efectos del clumping, nuestras determinaciones para las tasas de pérdida de masa son cotas superiores.

Para determinar los parámetros del viento, el primer paso fue buscar mediciones de $\mathrm{v}_{\infty}$ en el rango UV (Prinja et al. 2002, 1997, Howarth et al. 1997a y usar estos valores como iniciales en el proceso de ajuste para reproducir la línea $\mathrm{H}_{\alpha}$. Los valores de $\mathrm{v}_{\infty}$ calculados de nuestros ajustes se pueden encontrar en la tabla 3.3. Estos presentan desviaciones del $10 \%$ respecto a los valores medidos en el rango UV. En muy pocos casos la velocidad terminal derivada del espectro UV no fue capaz de ajustar el perfil de la línea observado en $\mathrm{H}_{\alpha}$, y sólo en esos casos las discrepancias con respecto a los datos del UV aumentan a un $30 \%$. Por lo tanto, consideramos un error relativo para $\mathrm{v}_{\infty}$ entre un $10 \%$ a un $30 \%$.

Considerando valores fijos en $v_{\infty}$ y en $R_{\star}$ observamos que el error en la tasa de pérdida de masa es diferente para los diferentes tipos espectrales. Esta observación recae en el hecho de que los anchos equivalentes de la componente en emisión del perfil P Cygni muestran cambios mayores al $20 \%$ cuando variamos $\dot{M}$ en un $10 \%$ y $20 \%$ para estrellas de tipos espectrales tardíos (B4-B9) y tempranos (B0-B1.5), respectivamente. Es decir que el error en $\dot{M}$ para las estrellas BSGs más frías es menor que para las más calientes. Sin embargo, cuando el perfil de línea de $\mathrm{H}_{\alpha}$ presenta una absorción pura, el error en $\dot{\mathrm{M}}$ puede ser mayor a un factor 2 (Markova et al.2004.

\subsection{Resultados}

Para cada estrella de nuestro programa modelamos los perfiles de línea de H, He y Si. La fig. 3.2 muestra el perfil de $\mathrm{H}_{\alpha}$ observado y el mejor modelo sintético que lo reproduce. El resto de las líneas y sus ajustes pueden encontrarse en la fig. 6 del apéndice. En la tabla 3.3 se listan los parámetros estelares y del viento que hemos encontrado con sus respectivos errores: $T_{\text {ef }}, \log g, \beta, \dot{M}, \mathrm{v}_{\infty}, \mathrm{v}_{\text {mic }}, \mathrm{v}_{\mathrm{mac}}, \mathrm{v} \sin \mathrm{i}, \mathrm{R}_{\star}, \log \mathrm{L} / \mathrm{L}_{\odot}, \log \mathrm{D}_{\mathrm{mom}}, \log \mathrm{L} / \mathrm{M}$ y $\mathrm{Q} . \mathrm{v}_{\text {mic }}$ y $\mathrm{v}_{\mathrm{mac}}$ son parámetros relacionados con las líneas fotosféricas. Inicialmente fijamos $\mathrm{v}_{\text {mic }}$ en $10 \mathrm{~km} / \mathrm{s}$ y en algunos casos dicho valor fue levemente modificado $( \pm 5 \mathrm{~km} / \mathrm{s})$ para obtener el mejor ajuste a las intensidades de las líneas de He y Si.

Recordemos que las líneas fotosféricas de cada estrella fueron modeladas con los mismos valores de $\mathrm{v}_{\text {mic }} \mathrm{y} \mathrm{v}_{\mathrm{mac}}$, siendo este último, por lo general, mayor que el primero. Sin embargo para ajustar correctamente la forma e intensidad de las líneas de $\mathrm{H}_{\alpha}$ y de $\mathrm{He} \mathrm{I} \lambda 6678$ A tuvimos que recurrir a otras velocidades de turbulencia ya que estas dos líneas están afectadas además por las velocidades de dispersión del fluido.

En lo que respecta a los parámetros del viento, podemos ver de la fig. 3.2 que hemos logrado reproducir con éxito la línea $\mathrm{H}_{\alpha}$ de muchas estrellas BSGs. En algunos casos, si bien se reproduce correctamente la emisión del perfil P Cygni, fue imposible ajustar la intensidad de la componente en absorción. Problemas de este tipo también se observan en los perfiles de $\mathrm{H}_{\alpha}$ en absorción de HD 38771, HD 75149 y HD 111973 debido a la presencia 

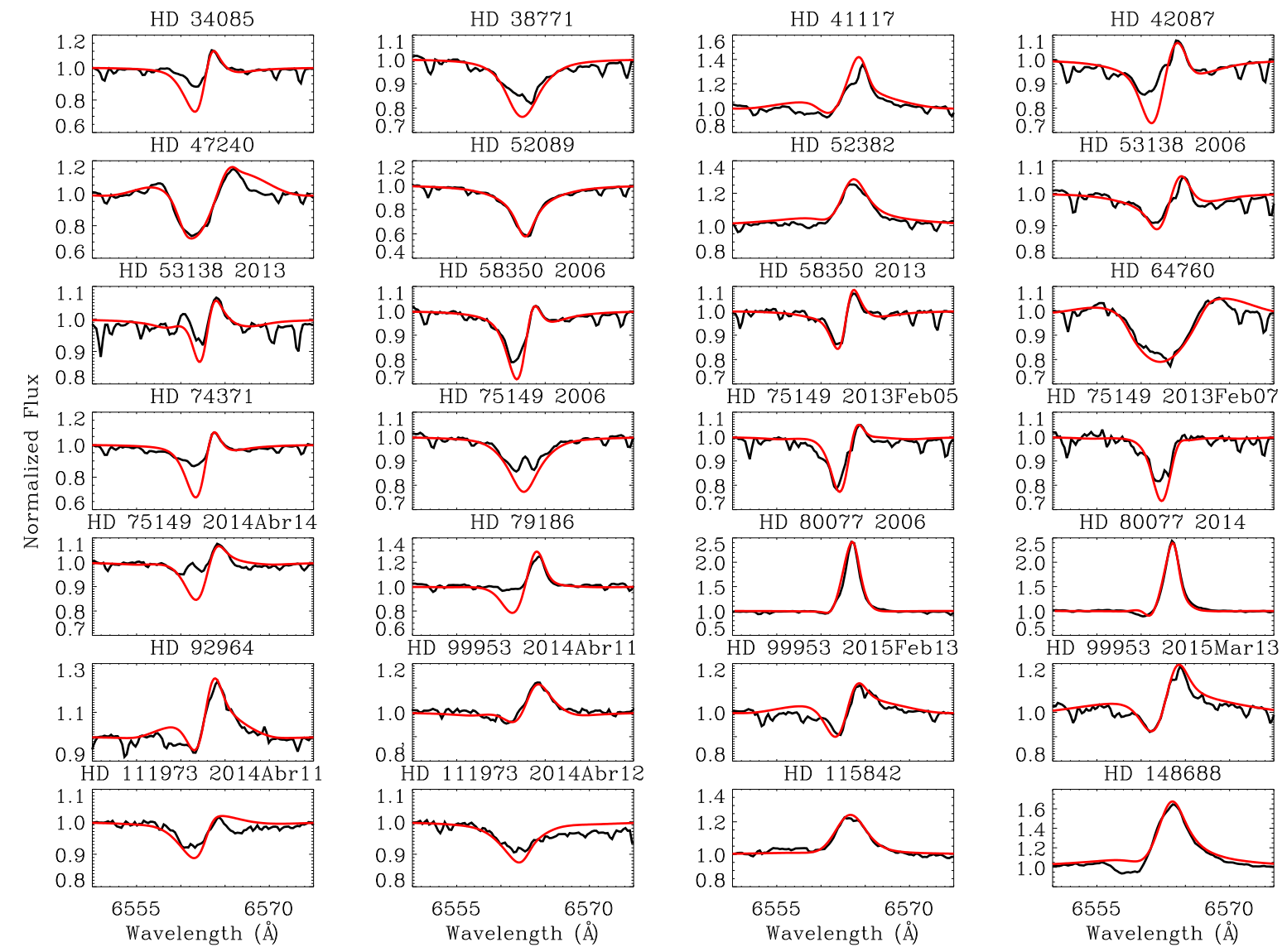

Figura 3.2. Ajustes de los perfiles observados de $\mathrm{H}_{\alpha}$ de todas las estrellas de la muestra. Cada panel representa el flujo normalizado en función de la longitud de onda. La observación y el modelo están caracterizados por una línea continua negra y roja, respectivamente.

de una incipiente emisión en el núcleo. En cambio, los perfiles sintéticos de las líneas fotosféricas reproducen muy bien las observaciones (ver secc. 6 del apéndice).

\subsubsection{Comentarios individuales sobre cada objeto}

En esta subsección comentaremos sobre las principales características encontradas en los objetos de estudio. Esta información estará complementada, en algunos casos, con imágenes tomadas con el satélite WISE (Wide-field Infrared Survey Explorer, Wright et al. 2010, el cual cubre el rango de $3.5 \mu$ a $22 \mu$ mediante cuatro bandas (W1 a W4). Estas imágenes fueron usadas para observar posibles fases previas de vientos estelares intensos, como los que suelen ocurrir en la fase de supergigante roja (Ekström et al. 2012, Saio et al. 2013a. Todos los parámetros se resumen en las tablas 3.1 y 3.3 .

\section{HD 34085 (B8Iae)}

Rigel es una estrella a la cual se le han dedicado numerosos trabajos. En éste en particular, 
Tabla 3.3. Parámetros estelares y del viento obtendidos por medio del ajuste de líneas sintéticas a las observadas.

\begin{tabular}{|c|c|c|c|c|c|c|c|c|c|c|c|c|c|}
\hline \begin{tabular}{|l|l} 
Estrella \\
\end{tabular} & $\begin{array}{c}T_{\text {ef }} \\
\mathrm{K}\end{array}$ & $\begin{array}{l}\log g \\
\operatorname{dex}\end{array}$ & $\bar{\beta}$ & $\begin{array}{c}M \\
10^{-6} \mathrm{M}_{\odot} / \mathrm{año}\end{array}$ & $\begin{array}{c}V_{\infty} \\
\mathrm{km} / \mathrm{s}\end{array}$ & $\begin{array}{l}\begin{array}{l}V_{\operatorname{mac}} \\
\mathrm{km} / \mathrm{s}\end{array} \\
\end{array}$ & $\begin{array}{c}V_{\text {mic }} \\
\mathrm{km} / \mathrm{s}\end{array}$ & $\begin{array}{l}V \sin i \\
\mathrm{~km} / \mathrm{s}\end{array}$ & $\begin{array}{l}R_{\star} \\
\mathrm{R}_{\odot}\end{array}$ & $\begin{array}{c}\log \left(L / L_{\odot}\right) \\
\operatorname{dex}\end{array}$ & $\begin{array}{c}\log D_{\text {mom }} \\
\text { dex }\end{array}$ & $\begin{array}{c}\log (L / M) \\
\operatorname{dex}\end{array}$ & $\begin{array}{c}\log Q \\
\operatorname{dex}\end{array}$ \\
\hline HD 34085 & $12700 \pm 500$ & $1,70 \pm 0,1$ & 2,6 & $0.23 \pm 0.02$ & $155 \pm 46$ & $52 \pm 3$ & $10 \pm 2$ & 30 & 72 & $5,09 \pm 0,09$ & $27,22 \pm 0,19$ & 4,13 & $-12,71 \pm 0,32$ \\
\hline HD 38771 & $25000 \pm 1000$ & $2,70 \pm 0,1$ & 1,5 & $0.14 \pm 0.04$ & $1500 \pm 150$ & $60 \pm 10$ & $13 \pm 2$ & 80 & 13 & $4,78 \pm 0,07$ & $27,69 \pm 0,19$ & 4,27 & $-13,29 \pm 0,24$ \\
\hline HD 41117 & $19000 \pm 1000$ & $2,30 \pm 0,1$ & 2,0 & $0.17 \pm 0.03$ & $510 \pm 51$ & $65 \pm 20$ & $10 \pm 5$ & 40 & 23 & $4,84 \pm 0,16$ & $27,38 \pm 0,15$ & 4,27 & $-12,78 \pm 0,23$ \\
\hline HD 42087 & $16500 \pm 1000$ & $2,45 \pm 0,1$ & 2,0 & $0.57 \pm 0.05$ & $700 \pm 70$ & $80 \pm 15$ & $15 \pm 5$ & 80 & 55 & $5,31 \pm 0,43$ & $28,27 \pm 0,13$ & 3,83 & $-13,12 \pm 0,23$ \\
\hline HD 47240 & $19000 \pm 1000$ & $2,40 \pm 0,1$ & 1,0 & $0.24 \pm 0.02$ & $450 \pm 90$ & $60 \pm 10$ & $10 \pm 3$ & 95 & 30 & $5,03 \pm 0,30$ & $27,57 \pm 0,15$ & 4,08 & $-12,82 \pm 0,26$ \\
\hline HD 52089 & $23000 \pm 1000$ & $3,00 \pm 0,1$ & 1,0 & $0.02 \pm 0.006$ & $900 \pm 270$ & $65 \pm 5$ & $8 \pm 2$ & 10 & 11 & $4,49 \pm 0,05$ & $26,58 \pm 0,28$ & 3,88 & $-13,69 \pm 0,38$ \\
\hline HD 52382 & $21500 \pm 1000$ & $2,45 \pm 0,1$ & 2,2 & $0.24 \pm 0.04$ & $1000 \pm 100$ & $65 \pm 5$ & $10 \pm 2$ & 55 & 21 & $4,94 \pm 0,32$ & $27,84 \pm 0,14$ & 4,29 & $-13,10 \pm 0,21$ \\
\hline \multirow[t]{2}{*}{ HD 53138} & $18000 \pm 1000$ & $2,25 \pm 0,2$ & 2,0 & $0.24 \pm 0.02$ & $600 \pm 120$ & $50 \pm 10$ & $10 \pm 4$ & 40 & 46 & $5,31 \pm 0,32$ & $27,79 \pm 0,14$ & 4,19 & $-13,09 \pm 0,22$ \\
\hline & $18000 \pm 1000$ & $2,25 \pm 0,2$ & 2,0 & $0.20 \pm 0.01$ & $450 \pm 135$ & $60 \pm 5$ & $9 \pm 3$ & 40 & 46 & $5,31 \pm 0,32$ & $27,59 \pm 0,14$ & 4,19 & $-13,36 \pm 0,22$ \\
\hline \multirow[t]{2}{*}{ HD 58350} & $15000 \pm 500$ & $2,00 \pm 0,1$ & 3,0 & $0.15 \pm 0.02$ & $233 \pm 23$ & $70 \pm 10$ & $12 \pm 2$ & 40 & 54 & $5,13 \pm 0,23$ & $27,21 \pm 0,13$ & 4,12 & $-12,97 \pm 0,20$ \\
\hline & $16000 \pm 500$ & $2,00 \pm 0,1$ & 3,0 & $0.12 \pm 0.01$ & $175 \pm 18$ & $50 \pm 10$ & $10 \pm 5$ & 40 & 54 & $5,24 \pm 0,23$ & $26,98 \pm 0,13$ & 4,23 & $-12,90 \pm 0,20$ \\
\hline HD 64760 & $23000 \pm 1000$ & $2,90 \pm 0,1$ & 0,5 & $0.42 \pm 0.06$ & $1500 \pm 150$ & $100 \pm 50$ & $15 \pm 5$ & 230 & 12 & $4,57 \pm 0,08$ & $28,14 \pm 0,12$ & 3,73 & $-12,76 \pm 0,18$ \\
\hline HD 74371 & $13700 \pm 500$ & $1,80 \pm 0,1$ & 2,0 & $0.28 \pm 0.03$ & $200 \pm 60$ & $60 \pm 10$ & $10 \pm 3$ & 30 & 73 & $5,23 \pm 0,19$ & $27,48 \pm 0,21$ & 4,17 & $-12,80 \pm 0,33$ \\
\hline \multirow{4}{*}{ HD 75149} & $16000 \pm 1000$ & $2,10 \pm 0,1$ & 2,5 & $0.09 \pm 0.03$ & $400 \pm 40$ & $62 \pm 12$ & $9 \pm 3$ & 40 & 61 & $5,35 \pm 0,29$ & $27,25 \pm 0,11$ & 4,14 & $-13,63 \pm 0,22$ \\
\hline & $16000 \pm 1000$ & $2,10 \pm 0,1$ & 2,5 & $0.20 \pm 0.01$ & $350 \pm 35$ & $52 \pm 12$ & $11 \pm 1$ & 40 & 61 & $5,35 \pm 0,29$ & $27,54 \pm 0,11$ & 4,14 & $9 \pm 0,22$ \\
\hline & $16000 \pm 1000$ & $2,10 \pm 0,1$ & 2,5 & $0.16 \pm 0.05$ & $400 \pm 40$ & $57 \pm 7$ & $17 \pm 1$ & 40 & 61 & $5,35 \pm 0,29$ & $27,50 \pm 0,11$ & 4,14 & $-13,37 \pm 0,22$ \\
\hline & $16000 \pm 1000$ & $2,10 \pm 0,1$ & 2,5 & $0.25 \pm 0.01$ & $350 \pm 35$ & $55 \pm 10$ & $15 \pm 1$ & 40 & 61 & $5,35 \pm 0,29$ & $27,64 \pm 0,11$ & 4,14 & $-13,10 \pm 0,22$ \\
\hline HD 79186 & $15800 \pm 500$ & $2,00 \pm 0,1$ & 3,3 & $0.40 \pm 0.02$ & $400 \pm 40$ & $53 \pm 7$ & $11 \pm 1$ & 40 & 61 & $5,33 \pm 0,38$ & $27,90 \pm 0,11$ & 4,21 & $-12,98 \pm 0,20$ \\
\hline \multirow{2}{*}{ HD 80077} & $17700 \pm 1000$ & $2,20 \pm 0,1$ & 3,2 & $5.4 \pm 0.50$ & $200 \pm 20$ & $\ldots$ & $\ldots$ & 10 & 195 & $6,53 \pm 0,17$ & $28,86 \pm 0,14$ & 4,23 & $-12,15 \pm 0,26$ \\
\hline & $17700 \pm 1000$ & $2,20 \pm 0,1$ & 3,0 & $5.4 \pm 0.50$ & $150 \pm 15$ & $60 \pm 5$ & $10 \pm 5$ & 10 & 195 & $6,53 \pm 0,17$ & $\pm 0,14$ & 4,23 & $-11,97 \pm 0,26$ \\
\hline 964 & $18000 \pm 1000$ & $2,20 \pm 0,1$ & 2,0 & $0.49 \pm 0.03$ & $370 \pm 55$ & $40 \pm 5$ & $11 \pm 1$ & 45 & 70 & $5,67 \pm 0,49$ & $27,98 \pm 0,13$ & 4,25 & $3 \pm 0,25$ \\
\hline \multirow[t]{3}{*}{ HD 99953} & $19000 \pm 1000$ & $2,30 \pm 0,1$ & 2,0 & $0.08 \pm 0.01$ & $250 \pm 50$ & $50 \pm 5$ & $18 \pm 2$ & 50 & 25 & $4,87 \pm 0,37$ & $26,80 \pm 0,14$ & 4,22 & $-12,79 \pm 0,24$ \\
\hline & $19000 \pm 1000$ & $2,30 \pm 0,1$ & 2,0 & $0,13 \pm 0,01$ & $500 \pm 100$ & $50 \pm 5$ & $18 \pm 2$ & 50 & 25 & $4,87 \pm 0,37$ & $27,33 \pm 0,14$ & 4,22 & $-13,03 \pm 0,24$ \\
\hline & $19000 \pm 1000$ & $2,30 \pm 0,1$ & 2,0 & $0,22 \pm 0,01$ & $700 \pm 140$ & $50 \pm 5$ & $18 \pm 2$ & 50 & 25 & $4,87 \pm 0,37$ & $27,69 \pm 0,14$ & 4,22 & $-13,02 \pm 0,24$ \\
\hline \multirow[t]{2}{*}{ HD 111973} & $16500 \pm 1000$ & $2,10 \pm 0,1$ & 2,0 & $0,21 \pm 0,01$ & $350 \pm 70$ & $57 \pm 8$ & $12 \pm 2$ & 35 & 46 & $5,16 \pm 0,20$ & $27,51 \pm 0,15$ & 4,19 & $-13,02 \pm 0,27$ \\
\hline & $16500 \pm 1000$ & $2,10 \pm 0,1$ & 2,0 & $0,14 \pm 0,004$ & $350 \pm 105$ & $63 \pm 3$ & $12 \pm 2$ & 35 & 46 & $5,16 \pm 0,20$ & $27,33 \pm 0,15$ & 4,19 & $-13,19 \pm 0,27$ \\
\hline HD 115842 & $25500 \pm 1000$ & $2,75 \pm 0,1$ & 2,5 & $1.80 \pm 0.30$ & $1700 \pm 340$ & $63 \pm 3$ & $23 \pm 7$ & 50 & 35 & $5,67 \pm 0,62$ & $29,06 \pm 0,18$ & 4,30 & $-12,91 \pm 0,28$ \\
\hline HD 148688 & $21000 \pm 1000$ & $2,45 \pm 0,1$ & 2,5 & $1.15 \pm 0.20$ & $1200 \pm 360$ & $65 \pm 5$ & $11 \pm 1$ & 50 & 31 & $5,23 \pm 0,28$ & $28,69 \pm 0,23$ & 4,26 & $-12,80 \pm 0,36$ \\
\hline
\end{tabular}

nosotros estimamos los siguientes parámetros estelares usando las líneas fotosféricas: $\mathrm{T}_{\mathrm{ef}}=$ $12700 \mathrm{~K}$ y $\log g=1.7$ dex. Del mejor ajuste de la SED, obtenido a partir de un modelo de Kurucz, encontramos $\mathrm{T}_{\mathrm{ef}}=11700 \mathrm{~K}, \log g=2.0 \mathrm{dex}, \mathrm{R}_{\star}=71 \mathrm{R}_{\odot}, E(B-V)=0.044 \mathrm{mag}$ y d $=259$ pc (este valor es próximo al valor de 264 pc medido por HIPPARCOS). Usando la distancia obtenida por HIPPARCOS y el valor mencionado de $E(B-V)$, estimamos que $\mathrm{M}_{\text {bol }}=-7.95 \mathrm{mag}$ y $\mathrm{R}_{\star}=70 \mathrm{R}_{\odot}$, mientras que usando el tamaño angular (2.713 mas, Zorec et al. 2009a calculamos $R_{\star}=76 \mathrm{R}_{\odot}$. Por lo tanto, hemos adoptado para Rigel un valor medio para el radio de $R_{\star}=72 R_{\odot}$.

Nuestra estimación para la $\mathrm{T}_{\text {ef }}(12700 \mathrm{~K})$ está de acuerdo con los valores calculados previamente por otros autores (ver tabla 6.1 en el apéndice). Se ha reportado que este objeto muestra variaciones fotométricas de 2.075 días (Lefèvre et al. 2009, y más aún, Moravveji et al. 2012 han encontrado 19 modos de pulsación a partir de la medición de velocidades radiales, cuyos períodos van desde 1.22 días a 74.47 días.

El viento estelar por su parte también presenta variaciones que se ven reflejadas en cambios en la tasa de pérdida de masa de al menos un $20 \%$ en escala de un año Chesneau et al. 2014. Estos autores han analizado esta estrella mediante datos espectro-interferométricos y han encontrado variaciones en la visibilidad diferencial y en las fases. También encuentran que para algunas épocas la evolución temporal de la señal sugiere la presencia de estructuras circunestelares en rotación. Sin embargo, en algunos períodos, no se observa ninguna señal. Estas observaciones han sido interpretadas en el contexto de perturbaciones de segundo orden en la esfericidad de la base del viento.

La línea $\mathrm{H}_{\alpha}$ es muy variable, mostrando perfiles en absorción pura y con emisiones en el núcleo, perfiles de doble pico o perfiles P Cygni invertidos (Chesneau et al. 2014. La ob- 
servación presentada en esta tesis (ver fig. 3.2) es similar a la reportada por Przybilla et al. 2006. Nuestra estimación de la tasa de pérdida de masa es $\sim 1.5$ veces más pequeña que la obtenida por Markova et al. 2008a y al menos un tercio del valor reportado por Chesneau et al. (2014). También hemos determinado un valor bajo de la velocidad terminal y resulta un tanto incierto ya que no hemos sido capaces de ajustar correctamente la componente en absorción del perfil $\mathrm{P}$ Cygni de $\mathrm{H}_{\alpha}$, mientras que para ajustar la componente en emisión se requirió un alto valor de $\mathrm{v}_{\mathrm{mac}}(85 \mathrm{~km} / \mathrm{s})$. Las líneas fotosféricas fueron modeladas correctamente con la excepción de la línea de He I $\lambda 4471$ que no exhibe la componente prohibida.

\section{HD 38771 (B0.5Ia)}

Esta estrella también ha sido estudiada por varios grupos de trabajo Nerney 1980 Garmany et al. 1981, Lamers et al. 1982, Kudritzki et al. 1999, Crowther et al. 2006a; Searle et al. 2008a: Zorec et al. 2009a) quienes obtuvieron parámetros que van de $26000 \mathrm{~K}$ a $27500 \mathrm{~K}$ en $\mathrm{T}_{\text {ef }}$, de 2.9 dex a 3.07 dex en $\log g$, de $13.0 \mathrm{R}_{\odot}$ a $28 \mathrm{R}_{\odot}$ en el radio estelar, de $0.27 \times 10^{-6} \mathrm{M}_{\odot} /$ año a $1.20 \times 10^{-6} \mathrm{M}_{\odot} /$ año en $\dot{\mathrm{M}}$, y de $1350 \mathrm{~km} / \mathrm{s}$ a $1870 \mathrm{~km} / \mathrm{s}$ en $\mathrm{v}_{\infty}($ ver tabla 6.1).

Nerney 1980 ha reportado la presencia de un campo magnético variable al igual que variaciones espectrales. El perfil de la línea $\mathrm{H}_{\alpha}$ es variable, presentando desde un perfil en absorción pura Kudritzki et al. 1999) a un perfil de doble pico con una emisión central Rusconi et al. 1980 y a una absorción con una fuerte emisión central Crowther et al. 2006a). Morel et al. 2004 también describen cambios en la morfología y amplitud del perfil de $\mathrm{H}_{\alpha}$ del $32.6 \%$, lo que claramente sugiere variaciones en las condiciones del viento. Estos últimos autores también encuentran dos períodos fotométricos de 4.76 días y 1.047 días mientras que Prinja et al. 2004 reportaron períodos espectroscópicos de 1.9, 6.5 y 9.5 días.

Al momento de nuestra observación la línea $\mathrm{H}_{\alpha}$ desplegó un perfil en absorción puro asimétrico con una pequeña emisión en el núcleo (ver fig. 3.2). También notamos que el núcleo de la línea $\mathrm{H}_{\beta}$ podría estar rellenado por una incipiente emisión (ver secc. 6] fig. 6.1. En todas las líneas fotosféricas hay presentes efectos extras de ensanchamiento que conducen a altos valores de $\mathrm{v}_{\operatorname{mac}}$ (del orden de la velocidad de rotación proyectada).

Derivamos una $\mathrm{T}_{\text {ef }}=25000 \mathrm{~K}$ usando el balance de ionización tanto de Si II y Si III como de HeI y HeII. El mejor ajuste a la SED fue obtenido con un modelo de TLUSTY para $\mathrm{T}_{\text {ef }}=25700 \mathrm{~K}, \log g=2.70 \mathrm{dex}, \mathrm{R}_{\star}=14 \mathrm{R}_{\odot}$ y $E(B-V)=0.05 \mathrm{mag}$, para una distancia de 191 pc (cercano al valor de HIPPARCOS: $\mathrm{d}_{\mathrm{H}}=198 \mathrm{pc}$, van Leeuwen 2007a). Usando $\mathrm{d}_{\mathrm{H}}$ en la fórmula de Pogson derivamos que $M_{\mathrm{bol}}=-6.99$ mag y $\mathrm{R}_{\star} \sim 12 \mathrm{R}_{\odot}$. El radio adoptado de $13 \mathrm{R}_{\odot}$ es consistente con el valor calculado a partir del tamaño angular por Zorec et al. 2009a. 0.62 mas).

Nuestro valor para la $T_{\text {ef }}$ es $\sim 1000 \mathrm{~K}$ menor que los valores obtenidos por Searle et al. 2008a y Gathier et al. 1981, mientras que el radio estelar es similar al obtenido por Kudritzki et al. (1999).

En lo que respecta a la pérdida de masa, si comparamos nuestra determinación con la de otros autores (ver tabla 6.1 en el apéndice), nuestro valor es menor, pero como el perfil de $\mathrm{H}_{\alpha}$ está en absorción pura, $\dot{\mathrm{M}}$ podría tener una gran incerteza. 
Considerando la diversidad de parámetros del viento encontrados en la literatura y partiendo del hecho que el perfil de $\mathrm{H}_{\alpha}$ muestra variaciones importantes, esta estrella es una buena candidata para investigar la posible existencia de una conexión entre pulsaciones o actividad magnética con las variaciones cíclicas del viento.

\section{HD 41117 (B2Ia)}

Esta es otra estrella profundamente estudiada como puede verse en los trabajos de Zorec et al. 2009a); Crowther et al. 2006a); Morel et al. 2004); Kudritzki et al. 1999); Scuderi et al. (1998); Nerney (1980). Morel et al. (2004) encontraron variaciones en $\mathrm{H}_{\alpha}$, tanto en la intensidad como en la forma de la línea, y reportaron un período de variación de 2.869 días.

De la tabla 6.1 del apéndice podemos ver que los parámetros fundamentales y la luminosidad que hemos obtenido son consistentes con aquellos ya publicados, en contraste con los parámetros del viento (principalmente $\dot{\mathrm{M}}$ ) que son diferentes.

El mejor ajuste a la SED corresponde a $\mathrm{T}_{\text {ef }}=17940 \mathrm{~K}, \log g=2.2 \mathrm{dex}, R_{\star}=26 \mathrm{R}_{\odot}$, $E(B-V)=0.42 \mathrm{mag}$ (en lugar de $E(B-V)=0.46$ mag que derivamos de Flower 1996) y d $=477 \mathrm{pc}$ (mientras que la distancia medida por HIPPARCOS es $552 \pm 85 \mathrm{pc}$ ). Por otro lado, usando $\mathrm{d}=447 \mathrm{pc}$ y el $E(B-V)$ calculado, se obtiene que $\mathrm{R}_{\star} \sim 23 \mathrm{R}_{\odot}$. A este mismo resultado se llega si usamos el diámetro angular (0.371 mas, Zorec et al. 2009a) o la $\mathrm{M}_{\text {bol }}$ computada (-7.35 mag). Por lo tanto, adoptamos $\mathrm{R}_{\star}=23 \mathrm{R}_{\odot}$ que es también consistente con el radio estelar que se estima a partir de la distancia de HIPPARCOS. Este valor adoptado para el radio estelar es menor que el derivado por otros autores (ver tabla 6.1. Sin embargo si usamos la distancia derivada por Megier et al. 2009, $1.62 \mathrm{kpc}$ ), obtenemos $R_{\star}=62 R_{\odot}$, que es el valor adoptado por otros autores.

Del modelado de las líneas determinamos valores de $\mathrm{T}_{\mathrm{ef}}=19500 \mathrm{~K}$ y $\log g=2.3$ dex. Nuestro espectro revela un perfil de $\mathrm{H}_{\alpha}$ en emisión con una compleja absorción desplazada hacia el azul (ver fig. 3.2 . La tasa de pérdida de masa derivada aquí es menor (por un factor 5) que el valor reportado por Kudritzki et al. 1999. También nuestro valor para la velocidad terminal es menor. El resto de las líneas presentan absorciones puras y fueron modeladas correctamente. Los valores obtenidos para $\mathrm{v}_{\text {mic }} \mathrm{y} \mathrm{v}_{\text {mac }}$ son comparables a la velocidad de rotación proyectada de la estrella ( $\mathrm{v} \sin \mathrm{i}=40 \mathrm{~km} / \mathrm{s}$ ). Un alto valor de $\mathrm{v}_{\text {mac }}$ $(\sim 65 \mathrm{~km} / \mathrm{s})$ también fue obtenido por Simón-Díaz \& Herrero 2014a.

\section{HD 42087 (B4Ia)}

La SED fue ajustada con un modelo de TLUSTY usando $\mathrm{T}_{\text {ef }}=15000 \mathrm{~K}, \log g=2.39$ dex, $\mathrm{R}_{\star}=60 \mathrm{R}_{\odot}, E(B-V)=0.34$ mag y d $=2021 \mathrm{pc}$ (que es similar a la distancia de 2075 pc derivada por Megier et al. 2009, a partir de las líneas de Ca II H+K). Por otro lado, una distancia $\mathrm{d}=2075 \mathrm{pc}$ arroja una $\mathrm{M}_{\mathrm{bol}}=-8.33$ mag y $\mathrm{R}_{\star}=50 \mathrm{R}_{\odot}$ mientras que el tamaño angular calculado por Zorec et al. 2009a arroja un $\mathrm{R}_{\star}=57 \mathrm{R}_{\odot}$. Adoptamos un valor medio de $R_{\star}=55 R_{\odot}$.

Nuestro espectro sólo cubre la región de $\mathrm{H}_{\alpha}$, por lo que no fuimos capaces de determinar la temperatura efectiva a partir del balance de ionización. Es por eso que adoptamos el valor obtenido por Zorec et al. 2009a $\left(\mathrm{T}_{\text {ef }}=16500 \mathrm{~K}\right)$ y derivamos de la línea de $\mathrm{H}$ un 
$\log \mathrm{g}=2.45$ dex. La $\mathrm{T}_{\text {ef }}$ es menor que la reportada por Benaglia et al. 2007) y Searle et al. 2008a, y mayor que la derivada del ajuste de la SED.

Esta estrella presenta variaciones significativas del $92 \%$ en $\mathrm{H}_{\alpha}$ y tanto esta línea como la de HeI $\lambda 6678 \AA$ presentan un comportamiento variable con una periodicidad de $\sim 25$ días (Morel et al. 2004). Este período es mayor que el de 6.807 días encontrado a partir de las curvas de luz de HIPPARCOS (Morel et al. 2004, Lefèvre et al. 2009).

Nuestra línea de $\mathrm{H}_{\alpha}$ despliega un perfil P Cygni cuya emisión es más bien débil y su contraparte en absorción pareciera estar ensanchada. Ajustamos este perfil usando $\dot{M}=$ $0.5710^{-6} \mathrm{M}_{\odot} /$ año y $\mathrm{v}_{\infty}=700 \mathrm{~km} / \mathrm{s}$. Esta $\mathrm{v}_{\infty}$ es similar a la obtenida en el rango UV (735 $\mathrm{km} / \mathrm{s}$, Howarth et al. 1997a.

\section{HD 47240 (B1I)}

Es una estrella con alta rotación (v sin $i=114 \mathrm{~km} / \mathrm{s}$, Simón-Díaz \& Herrero 2014a). Presenta líneas fotosféricas en absorción muy ensanchadas, variaciones periódicas de luz de 2.742 días (Lefèvre et al. 2009 Morel et al. 2004), movimientos periódicos, ya que es un sistema binario, y presenta componentes de absorción discretas (DACs, Prinja et al.|2002). Obtuvimos un correcto ajuste de la SED con los siguientes parámetros: $\mathrm{T}_{\mathrm{ef}}=17500 \mathrm{~K}$, $\log g=2.4 \mathrm{dex}, E(B-V)=0.33 \mathrm{mag}, \mathrm{d}=1515 \mathrm{pc} \mathrm{y} \mathrm{R}_{\star}=35 \mathrm{R}_{\odot}$. La distancia es consistente con la estimación de 1598 pc derivada por Megier et al. 2009 y el exceso de color ajusta la depresión observada en el espectro continuo en $2200 \AA$. También calculamos una $\mathrm{M}_{\text {bol }}=-7.66$ mag y $\mathrm{R}_{\star}=28 \mathrm{R}_{\odot}$ (para $\mathrm{d}=1598 \mathrm{pc}$ ). Este radio estelar está de acuerdo con el obtenido a partir del tamaño angular (0.157 mas) encontrado por Zorec et al. 2009a. Adoptamos, por lo tanto, un valor de $\mathrm{R}_{\star}=30 \mathrm{R}_{\odot}$.

Los parámetros del viento calculados con FASTWIND concuerdan con los valores obtenidos por Lefever et al. 2007). Estos autores encuentran dos modelos con valores de $T_{\text {ef }}$ muy diferentes y ambos ajustan muy bien los perfiles de Si III. La $\mathrm{T}_{\text {ef }}$ obtenida por nosotros es menor que el valor de $21670 \mathrm{~K}$ derivado con el método BCD Zorec et al.2009a.

Para modelar las líneas fotosféricas fue necesario considerar un valor alto de $\mathrm{v}_{\text {mac }}(60$ $\mathrm{km} / \mathrm{s}$ ) que es bastante más alto que el usado para modelar las líneas de $\mathrm{H}$ formadas en el viento $(3 \mathrm{~km} / \mathrm{s})$. Como este objeto es una variable pulsante del tipo $\alpha$ Cyg, es esperable este valor alto de $\mathrm{v}_{\mathrm{mac}}$.

Nuestro espectro muestra un perfil $\mathrm{H}_{\alpha}$ con doble pico en emisión y con una absorción central muy marcada. Este perfil fue considerado como un indicador de la presencia de una estructura tipo disco (Lefever et al. 2007). Nuestro modelo ajusta a la observación tanto en su absorción como en sus componentes en emisión. Derivamos una tasa de pérdida de masa de $2.4 \times 10^{-7} \mathrm{M}_{\odot} /$ año. Este valor concuerda con el valor superior obtenido por Lefever et al. 2007 $1.7 \times 10^{-7} \mathrm{M}_{\odot} /$ año $-2.4 \times 10^{-7} \mathrm{M}_{\odot} /$ año), y es menor al valor teórico calculado por Morel et al. 2004 $\left.0.31 \times 10^{-7} \mathrm{M}_{\odot} / \mathrm{año}\right)$. En lo que respecta a la velocidad terminal, encontramos que nuestro valor es la mitad del determinado por Lefever et al. 2007) y Prinja \& Massa 2013.

\section{HD 52089 (B1.5II)}

Es el objeto de la muestra más brillante en el extremo UV, mostrando emisiones también 
en rayos-X consistentes con la presencia de choques (Kholtygin et al. 2015). Usando la SED observada y un modelo de TLUSTY derivamos $\mathrm{T}_{\mathrm{ef}}=21000 \mathrm{~K}, \log g=3.0$ dex y $\mathrm{R}_{\star}=11$ $\mathrm{R}_{\odot}$, para una distancia de $124 \mathrm{pc}$ van Leeuwen2007a y un $E(B-V)=0.00$ mag. Este radio estelar es también consistente con la magnitud bolométrica $\left(\mathrm{M}_{\mathrm{bol}}=-6.18 \mathrm{mag}\right)$ que obtuvimos y la medición del diámetro angular (0.801 mas, Zorec et al. 2009a que conduce a un valor de $10 \mathrm{R}_{\odot}$ y $10.6 \mathrm{R}_{\odot}$, respectivamente. De FASTWIND derivamos una $\mathrm{T}_{\text {ef }}$ mayor (23000 K) que está en acuerdo con los valores dados en Lefever et al. 2010); Zorec et al. 2009a y Morel et al. 2008, pero es mayor que los $20100 \mathrm{~K}$ obtenidos por Fraser et al. (2010). Nuestra determinación de la gravedad superficial (3 dex) está más cerca del valor reportado por Fraser et al. 2010 que los valores determinados por Lefever et al. (2010) y Morel et al. 2008), que son 0.2 dex menor.

La estrella presenta un campo magnético longitudinal de 149 G Morel et al. 2008. Nuestro espectro muestra todas las líneas de $\mathrm{H}$ en absorción y el valor de $\mathrm{M}$ calculado es de $2 \times 10^{-8} \mathrm{M}_{\odot} /$ año, que es similar al valor obtenido fenomenológicamente por Cohen et al. $1996,3 \times 10^{-8} \mathrm{M}_{\odot} /$ año $-8 \times 10^{-8} \mathrm{M}_{\odot} /$ año).

\section{HD 52382 (B1Ia)}

Usando FASTWIND para ajustar los cocientes de Si III/Si II y HeII/HeI obtenemos una $\mathrm{T}_{\text {ef }}=21500 \mathrm{~K}$, y de las líneas de $\mathrm{H} \gamma$ y $\mathrm{H} \delta$ un $\log g=2.45$ dex. De Flower 1996) obtuvimos $(B-V)_{0}=-0.20$ mag y se derivó un exceso de color de 0.39 mag. El mejor ajuste de la SED fue mediante un modelo de TLUSTY con $\mathrm{T}_{\mathrm{ef}}=23140 \mathrm{~K}, \log g=2.47 \mathrm{dex}, \mathrm{R}_{\star}=21.6 \mathrm{R}_{\odot}$ y d $=1.23 \mathrm{kpc}$, pero para poder ajustar la depresión de los $2200 \AA$ necesitamos un valor mayor del exceso de color: $E(B-V)=0.44 \mathrm{mag}$. La distancia obtenida concuerda con el valor de $1.1 \mathrm{kpc}$ encontrado por Megier et al. (2009) y con la distancia paraláctica de 1.3 kpc medida por Gaia (0.768 mas, Astraatmadja \& Bailer-Jones 2016). Usando este valor de $1.3 \mathrm{kpc}$ en la fórmula de Pogson y el radio angular (de 0.17 mas) dado por Pasinetti Fracassini et al. 2001) obtenemos radios de $18.6 \mathrm{R}_{\odot}$ y $23.5 \mathrm{R}_{\odot}$, respectivamente. Por lo cual adoptamos $\mathrm{R}_{\odot}=21 \mathrm{R}_{\odot}$ como valor medio. No tuvimos en cuenta la distancia medida por HIPPARCOS (de $471 \mathrm{pc}$ ) porque conlleva a un valor muy bajo de $\mathrm{M}_{\text {bol }}$ que no es consistente con el valor esperado para una estrella BSG.

El valor de $21000 \mathrm{~K}$ para la $\mathrm{T}_{\text {ef }}$ derivado por nosotros está entre los valores calculados por Lefever et al. 2010 y Krtička \& Kubát 2001a.

La estrella presenta un perfil de $\mathrm{H}_{\alpha}$ variable desplegando un perfil P Cygni (Morel et al. 2004 o una emisión pura. Nuestro espectro muestra un emisión pura y logramos reproducir muy bien todas las líneas. Derivamos una velocidad terminal de $1000 \mathrm{~km} / \mathrm{s}$ que es del mismo orden que la medida en el UV $(900 \mathrm{~km} / \mathrm{s}$, Prinja et al. 1990a) pero $200 \mathrm{~km} / \mathrm{s}$ menor que la encontrada por Krtička \& Kubát 2001a para una pérdida de masa similar.

La imagen combinada de WISE en sus bandas W2 $(4.6 \mu \mathrm{m})$, W3 $(12 \mu \mathrm{m})$ y W4 (22 $\mu \mathrm{m})$ revela una compleja estructura cercana a la estrella y en la misma dirección que su movimiento propio (indicado con una flecha blanca en la fig. 3.3 que podría estar relacionada con interacciones previas entre el viento de la estrella y el medio interestelar debido a intensos episodios de pérdida de masa.

Queremos recalcar que para este objeto también se requirió un valor alto de $\mathrm{v}_{\text {mac }}$ para 


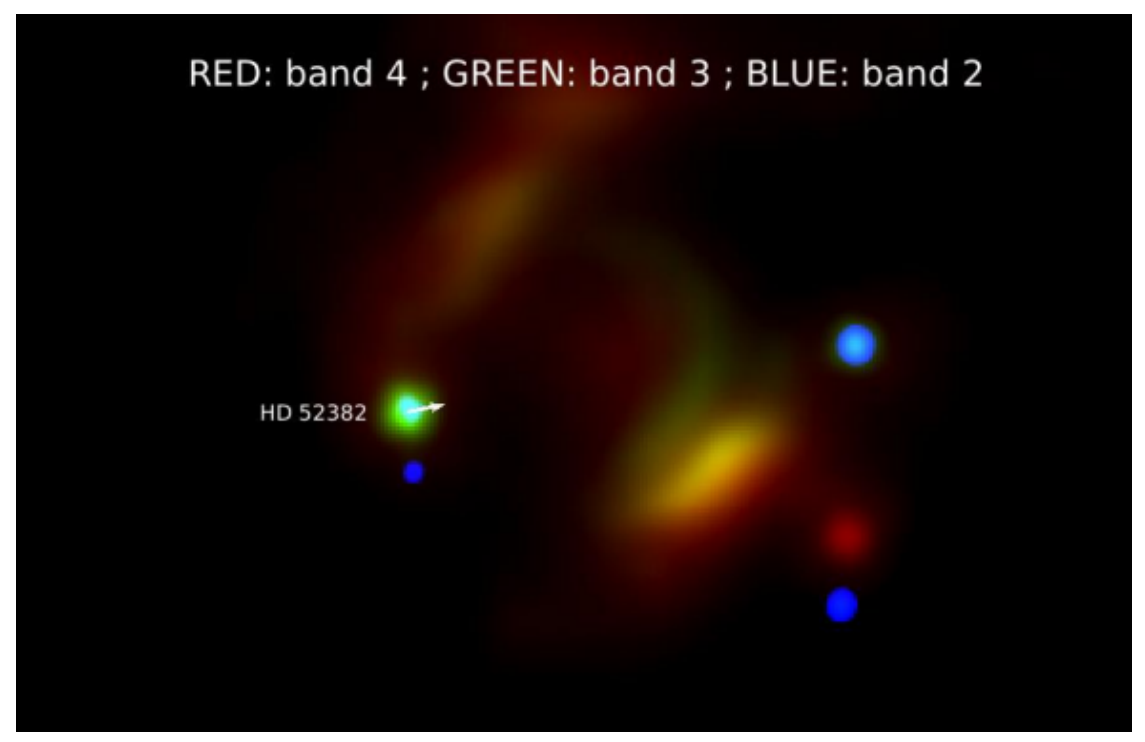

Figura 3.3. Imagen de WISE (W2 $=4.6 \mu \mathrm{m}$ en azul, $\mathrm{W} 3=12 \mu \mathrm{m}$ en verde, $\mathrm{y}$ W $4=22 \mu \mathrm{m}$ en rojo) mostrando una estructura de arco de choque cerca del objeto HD 52382. La flecha blanca indica la dirección del movimiento propio de la estrella.

ajustar correctamente las líneas fotosféricas (alrededor de $65 \mathrm{~km} / \mathrm{s}$ ). El mismo comportamiento fue reportado por Lefever et al. 2010 y podría ser un indicador de actividades de pulsación.

La línea He I $\lambda 6678$ Å presenta una pequeña emisión en el núcleo (ver secc. 6. fig. 6.2.

\section{HD 53138 (B3Ia)}

Esta estrella ha sido estudiada en varios trabajos (ver tabla 6.1) y las $T_{\text {ef }}$ derivadas van desde $15400 \mathrm{~K}$ a $18500 \mathrm{~K}$, mientras que la gravedad superficial va de 2.05 dex a 2.35 dex.

El objeto exhibe variaciones irregulares en $\mathrm{H}_{\alpha}$ (Morel et al.2004), y nuestros espectros también muestran variaciones. En 2006 la estrella presentó un perfil P Cygni mientras que en 2013 se observó un perfil con doble pico en emisión (ver fig. 3.2). También notamos diferencias en las intensidades y anchos de las líneas de $\mathrm{H}_{\gamma}$ y Si III entre las dos épocas, lo que nos condujo a obtener pequeñas diferencias entre la $\mathrm{T}_{\text {ef }} \mathrm{y} \log \mathrm{g}$ de una y otra época de observación. Obtenemos así, para las dos épocas, los siguientes parámetros fundamentales: $18000 \mathrm{~K}$ para $\mathrm{T}_{\text {ef }} \mathrm{y} 2.25$ dex para log $g$ con incertezas de $1000 \mathrm{~K}$ y 0.2 dex, respectivamente. El mejor ajuste para la SED sugiere que $\mathrm{T}_{\text {ef }}=18000 \mathrm{~K}, \log g=2.2 \mathrm{dex}, \mathrm{R}_{\star}=46 \mathrm{R}_{\odot}$, d $=822 \mathrm{pc}$ (cerca de los $847 \mathrm{pc}$ medidos por HIPPARCOS) y $E(B-V)=0.10 \mathrm{mag}$ (exceso de color menor que 0.131 mag obtenido de Flower 1996). El cálculo de $R_{\star}$ por medio del tamaño angular deriva en $51 \mathrm{R}_{\odot}$, mientras que el computado a partir de $\mathrm{M}_{\text {bol }}$ (-8.53 mag) sugiere que $R_{\star}=46 R_{\odot}$. Adoptamos, por lo tanto, un $R_{\star}=46 R_{\odot}$.

Las líneas fotosféricas están ensanchadas por macroturbulencia $\left(\mathrm{v}_{\mathrm{mac}}=60 \mathrm{~km} / \mathrm{s}\right)$ tienen casi el doble que la velocidad de rotación proyectada medida. Esta estrella ha sido clasificada como una variable pulsante del tipo $\alpha$ Cyg por Lefèvre et al. 2009. 
Nuestros modelos ajustan bien la línea observada de He I $\lambda 4471 \AA$ y la componente en emisión de $\mathrm{H}_{\alpha}$, aunque falla al querer ajustar la componente en absorción en las distintas épocas. El valor de la pérdida de masa que estimamos está en el rango de los valores previamente determinados por otros autores, siendo mayor en 2006. La velocidad terminal medida en el espectro de 2013 es menor que la correspondiente al 2006 (ver tabla 6.1). Barlow \& Cohen 1977) midieron un significativo exceso infrarrojo en $10 \mu \mathrm{m}$ sugiriendo una pérdida de masa con un factor 20 veces mayor al estimado en nuestro trabajo.

\section{HD 58350 (B5I)}

El conjunto de parámetros estelares $\left(\mathrm{T}_{\text {ef }} \mathrm{y} \log \mathrm{g}\right.$ ) que derivamos con FASTWIND concuerda muy bien con aquellos obtenidos por McErlean et al. 1999a); Searle et al. 2008a); Fraser et al. 2010). En este caso no fuimos capaces de obtener un buen ajuste de la SED usando fotometría UV, visual e IR. De todo modos, basados en la distancia medida por HIPPARCOS (609 pc) y la calculada con el diámetro angular (0.882 mas, Zorec et al. 2009a, obtenemos un $\mathrm{R}_{\star}=57 \mathrm{R}_{\odot}$. Basándonos en la paralaje de HIPPARCOS y el exceso de color $(E(B-V)=0.083 \mathrm{mag})$ derivado de datos obtenidos por Flower (1996), obtenemos que $\mathrm{M}_{\mathrm{bol}}=-7.97 \mathrm{mag}$ y $\mathrm{M}_{\star}=51 \mathrm{R}_{\odot}$. Adoptamos así, el valor medio $\mathrm{R}_{\star}=54 \mathrm{R}_{\odot}$.

$\mathrm{H}_{\alpha}$ muestra un perfil P Cygni variable con una pequeña componente en emisión. $\mathrm{Pu}-$ dimos modelar correctamente la observación tomada en 2006 y lograr un buen ajuste a la de 2013. En esta última fallamos en reproducir la componente en absorción. La tasa de pérdida de masa que determinamos es similar a la medida por Lefever et al. 2007) y menor que el valor obtenido por Searle et al. 2008a). Como estos últimos autores no muestran el espectro estelar en la región de $\mathrm{H}_{\alpha}$, no podemos discutir el origen de las discrepancias. Morel et al. 2004 muestra series temporales de $\mathrm{H}_{\alpha}$ donde el perfil es observado en absorción mientras que las observaciones de Ebbets 1982 muestran un perfil del tipo P Cygni. La curva de luz presenta un período de 4.7 días (Koen \& Eyer 2002 y otro de 6.631 días (Lefever et al. 2007).

\section{HD 64760 (B0.5Ib)}

Es un rotador rápido al que se le dedicó una "mega campaña" de observación con el IUE Massa et al. 1995 Prinja et al. 1995. La variabilidad de las líneas observadas en el UV argumentan a favor de una variación del viento modulada por rotación.

Despliega un perfil con doble pico en emisión en $\mathrm{H}_{\alpha}$ y líneas de absorción muy anchas de $\mathrm{H}$ y He. Las observaciones revelaron una conexión directa entre pulsaciones no radiales multiperiódicas y vientos espacialmente estructurados (Kaufer et al. 2006). Estas observaciones también son compatibles con la presencia de regiones interactuantes en rotación (CIRs, ver secc 2.9.2).

El ajuste de la SED nos provee los siguientes parámetros: $\mathrm{T}_{\mathrm{ef}}=22370 \mathrm{~K}, \log g=$ 2.50 dex, $\mathrm{R}_{\star}=15 \mathrm{R}_{\odot}, E(B-V)=0.07$ mag y d= $486 \mathrm{pc}$ (mientras que la paralaje de HIPPARCOS da $d=507 \mathrm{pc}$ ). La $\mathrm{M}_{\text {bol }}$ derivada es de -6.72 mag y arroja un $\mathrm{R}_{\star}=12 \mathrm{R}_{\odot}$, mientras que el tamaño angular da $R_{\star}=10 R_{\odot}$. Por lo tanto, adoptamos $R_{\star}=12 R_{\odot}$ como valor medio.

Nuestros parámetros estelares derivados con FASTWIND $\left(\mathrm{T}_{\mathrm{ef}}=23000 \mathrm{~K}, \log g=2.90\right.$ 


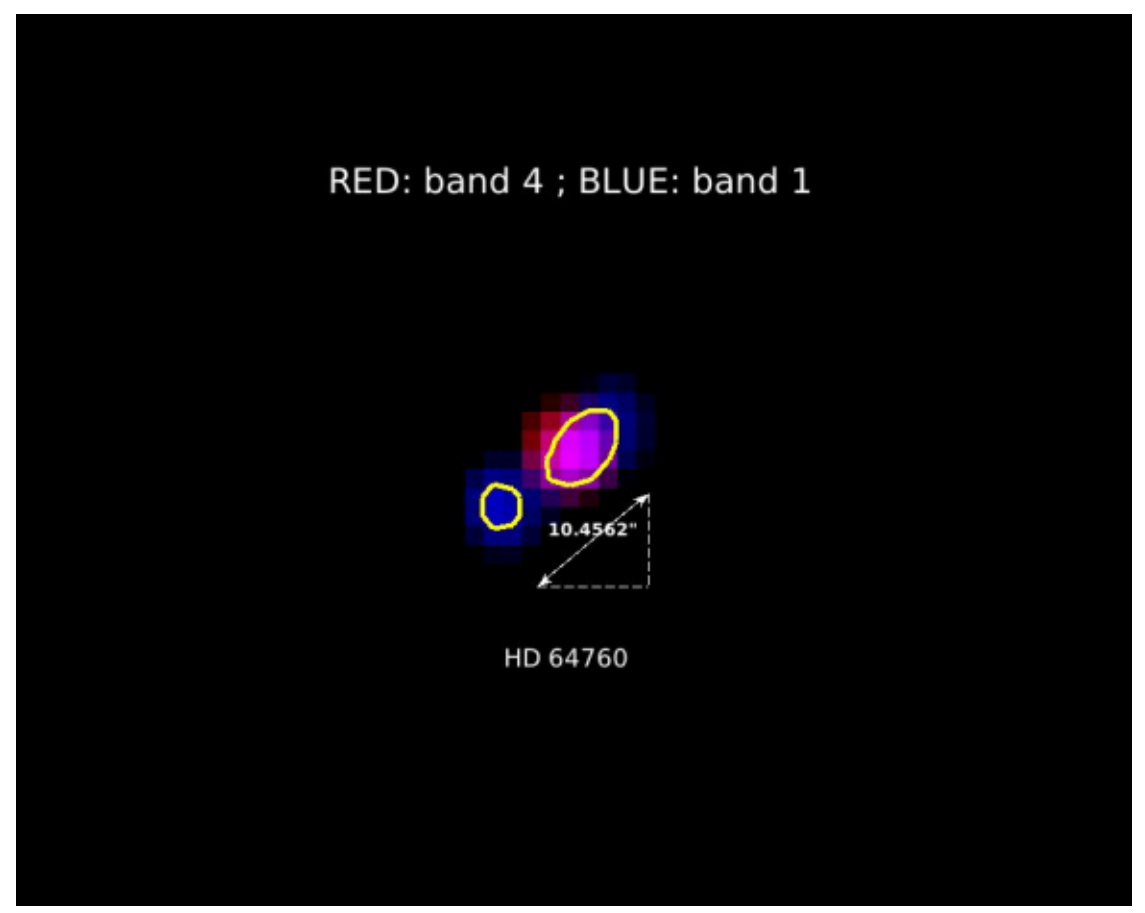

Figura 3.4. Imagen combinada de WISE en sus bandas W1 y W4 (W1 $=3.4 \mu \mathrm{m}$ en azul y $\mathrm{W} 4=22 \mu \mathrm{m}$ en rojo) mostrando la doble componente o estructura tipo lóbulo en HD 64760. Las curvas amarillas corresponden a un mismo brillo superficial. La flecha blanca indica la separación angular de la segunda componente o lóbulo (10.45"), la cual es mayor que la resolución del satélite en la banda W1 (6.1").

dex) están de acuerdo aquellos determinados por Lefever et al. (2007) pero existe una gran discrepancia con los cálculos realizados por Searle et al. 2008a). Aunque Searle et al. 2008a no muestra el espectro, estos autores mencionan algunas dificultades a la hora de derivar valores precisos a partir del modelado debido al gran ensanchamiento que presentan las líneas espectrales.

En general, obtuvimos muy buenos ajustes de todas las líneas, incluso para el perfil con doble pico en emisión de $\mathrm{H}_{\alpha}$. Nuestro modelado brinda el mismo conjunto de valores para los parámetros del viento calculados por Lefever et al. 2007), pero en lo que respecta a los parámetros estelares, el radio derivado en el presente trabajo es la mitad del valor obtenido por estos autores.

La imagen combinada de WISE en sus bandas W1 y W4 muestra la presencia de una doble componente o una estructura tipo lóbulo (fig. 3.4).

HD 74371 (B6Iab/b)

Esta estrella BSG despliega variaciones en su curva de luz con períodos de 5-20 días van Genderen et al. 1989) y 8.291 días (Koen \& Eyer 2002, Lefèvre et al. 2009). Los parámetros estelares $\mathrm{T}_{\text {ef }}$ y $\log g$ determinados por Fraser et al. (2010) están de acuerdo con los valores que obtuvimos con FASTWIND ( $\left.\mathrm{T}_{\mathrm{ef}}=13700 \mathrm{~K}, \log g=1.8 \mathrm{dex}\right)$. De la SED no fuimos 
capaces de derivar un buen ajuste usando la paralaje de HIPPARCOS $(0.43$ mas, $\mathrm{d}=$ $2.3 \mathrm{kpc}$ van Leeuwen 2007a). El mejor ajuste arroja una distancia menor de $1.8 \mathrm{kpc}$, un $E(B-V)=0.35, \mathrm{~T}_{\mathrm{ef}}=13800 \mathrm{~K}, \log g=2.0$ dex y $\mathrm{R}_{\star}=73 \mathrm{R}_{\odot}$. Este valor para la distancia de $1.8 \mathrm{kpc}$ es muy similar al reportado por Humphreys (1978, $1.9 \mathrm{kpc}$ ).

Nuestro modelo ajusta muy bien todas las líneas espectrales con la excepción de la componente en absorción de $\mathrm{H}_{\alpha}$ y el núcleo de $\mathrm{H}_{\beta}$, que parece estar rellenado por una incipiente emisión (ver fig. 6.4). Esta es la primera determinación de los parámetros del viento de HD 74371.

\section{HD 75149 (B3Ia)}

Los parámetros estelares obtenidos con FASTWIND son muy similares a los reportados por Lefever et al. 2007), Fraser et al. 2010 y con el modelado de la SED para $\mathrm{T}_{\text {ef }}=$ $15000 \mathrm{~K}, \log g=2.12 \mathrm{dex}, \mathrm{R}_{\star}=71 \mathrm{R}_{\odot}, E(B-V)=0.46$ mag y una distancia de 1642 pc. No se pudo ajustar correctamente la SED usando la paralaje medida por HIPPARCOS de 0.37 mas $(2.7 \mathrm{kpc})$. La distancia que derivamos, de $1.64 \mathrm{kpc}$, conlleva a una $\mathrm{M}_{\mathrm{V}}=-7.05$ mag, que está de acuerdo con el valor calculado por Humphreys 1978, -7.0 mag). Además obtuvimos $\mathrm{M}_{\text {bol }}=-8.45 \mathrm{mag}$ y $\mathrm{R}_{\star}=56 \mathrm{R}_{\odot}$. Como el diámetro angular arroja $\mathrm{R}_{\star}=58 \mathrm{R}_{\odot}$, adoptamos $R_{\star}=61 R_{\odot}$ como un valor promedio.

Esta estrella despliega una pequeña amplitud de variabilidad en su curva de luz con un período de 1.086 días (Koen \& Eyer 2002 Lefèvre et al. 2009, sumados a los períodos de variación de 1.2151 días y 2.2143 días reportados por Lefever et al. 2007.

De nuestro espectro encontramos que este objeto muestra variaciones importantes en la línea $\mathrm{H}_{\alpha}$ : un perfil en absorción pura con una pequeña emisión en el núcleo fue observado en 2006, mientras que un perfil P Cygni apareció el 5 de febrero de 2013, el cual se convirtió en una compleja absorción dos días después (ver figs. 6.4 y 6.5. Este útimo perfil también presenta dos componentes débiles en emisión que se asemejan a las publicadas por Lefever et al. 2007. Usando el espectro tomado en 2006, el valor determinado de $\dot{M}$ es similar al valor reportado por estos autores, aunque nuestro valor de $\mathrm{v}_{\infty}$ es un poco menor. Para explicar las variaciones del perfil de línea de $\mathrm{H}_{\alpha}$ tuvimos que considerar una estructura de viento variable con un aumento en la tasa de pérdida de masa alrededor de un factor $1.8 \mathrm{y}$ 2.2 entre las observaciones de 2013 y 2014, y un factor 2.8 entre los datos de 2014 y 2006. Es importante remarcar que, para modelar la línea de $\mathrm{H}_{\alpha}$ de cada época, fue necesario considerar diferentes valores (y también grandes) de $\mathrm{v}_{\text {mac }}$. Los valores determinados para la velocidad terminal son muy similares en todas las épocas. Sin embargo, hay que tener en cuenta que los valores determinados en los casos de los perfiles de absorción puros, involucran una incerteza mayor que el resto ya que una línea con esta característica es menos sensible a las condiciones del viento.

El resto de las líneas fueron modeladas correctamente con la excepción del núcleo de la línea $\mathrm{H}_{\beta}$ observada en 2006.

\section{HD 79186 (B5Ia)}

La SED fue ajustada con un modelo TLUSTY usando los siguientes parámetros: $\mathrm{T}_{\text {ef }}=$ $15000 \mathrm{~K}, \log g=2.12 \mathrm{dex}, \mathrm{R}_{\star}=67 \mathrm{R}_{\odot}, \mathrm{y} \mathrm{d}=1.42 \mathrm{kpc}$, adoptando $E(B-V)=0.35$ mag. 
La distancia derivada es muy similar a la obtenida con la paralaje de HIPPARCOS (1.45 kpc), que arroja una $M_{\text {bol }}=-8.26$ mag y un $R_{\star}=53 R_{\odot}$, mientras que del tamaño angular (0.4 mas) se obtiene $61 \mathrm{R}_{\odot}$. Adoptamos un valor promedio de $61 \mathrm{R}_{\odot}$.

Nuestra estimación de $\mathrm{T}_{\text {ef }}$ es apenas mayor que los valores dados por Fraser et al. 2010) y Prinja \& Massa 2010a): con $\Delta \mathrm{T} \sim 700 \mathrm{~K}$ y $\Delta \mathrm{T} \sim 800 \mathrm{~K}$, respectivamente, y mucho mayor que los obtenidos por Krtička \& Kubát 2001a y Underhill 1984. El logaritmo de la gravedad superficial concuerda con el único valor encontrado en la literatura de 2.0 dex Fraser et al. 2010. El radio estelar de nuestro modelo es similar al estimado por Underhill (1984).

Aunque pudimos ajustar muy bien todas las líneas fotosféricas, no fue el caso de $\mathrm{H}_{\alpha}$, para la cual el modelo no reproduce la componente en absorción del perfil P Cygni. Sin embargo, la velocidad terminal obtenida concuerda con el valor publicado por Krtička \& Kubát 2001a y con las mediciones UV $(435 \mathrm{~km} / \mathrm{s})$ determinadas por Prinja \& Massa 2010a. Nuestro valor de $\dot{M}$ es un poco menor que el obtenido por Krtička \& Kubát 2001a).

\section{HD $80077(\mathrm{~B} 2 \mathrm{Ia}+\mathrm{e})$}

Puede ser un miembro del cúmulo abierto Pismis 11, localizado a una distancia de $3.6 \mathrm{kpc}$. Con una magnitud absoluta de -10.4 mag es una de las BSGs más brillante de nuestra Galaxia Knoechel \& Moffat 1982 Marco \& Negueruela 2009. Carpay et al. 1989, 1991) detectaron variaciones de luz con una amplitud de $\sim 0.2$ mag y sugirieron que la estrella podría ser una variable azul luminosa (LBV, siglas en inglés). Usando datos de HIPPARCOS y datos fotométricos, van Leeuwen et al. (1998) obtuvieron un periodograma que reveló que los picos más significativos son aquellos correspondientes a períodos de 66.5 días y 55.5 días, y otros dos picos de luz, menos significativos se observan con períodos de 76 días y 41.1 días. Lefèvre et al. 2009 encontraron variaciones de luz de 0.151 mag en un período de 3.115 días. Otro período de 21.2 días fue determinado mediante polarimetría por Knoechel \& Moffat 1982.

Del ajuste de la SED obtuvimos $\mathrm{T}_{\text {ef }}=18000 \mathrm{~K}, \log g=2.17 \mathrm{dex}, E(B-V)=1.5$ mag, $R_{\star}=200 \mathrm{R}_{\odot} \mathrm{y} \mathrm{d}=3600 \mathrm{pc}$ (mientras la distancia derivada de HIPPARCOS es 877 pc). Usando la distancia a Pismis 11 y el exceso de color obtenido, calculamos una $\mathrm{M}_{\mathrm{bol}}=$ -11.49 mag, y $\mathrm{R}_{\star}=187 \mathrm{R}_{\odot}$. Hemos adoptado para esta estrella un valor del radio estelar de $195 \mathrm{R}_{\odot}$. Carpay et al. (1989) derivaron $\mathrm{T}_{\text {ef }}=17700 \mathrm{~K}, \log g=2$ dex, y una tasa de pérdida de masa de $5.11 \times 10^{-6} \mathrm{M}_{\odot} /$ año. Por otro lado, Benaglia et al. 2007 estimaron un valor significativamente menor de $\dot{M}\left(1.7 \times 10^{-6} \mathrm{M}_{\odot} /\right.$ año $)$ que concuerda con nuestro resultado (ver tabla 6.1).

Observamos un perfil P Cygni en $\mathrm{H} \alpha$ en los espectros tomados en 2006 y 2014, habiendo muy pocos cambios en las emisiones de uno y otro espectro (ver fig. 3.2.

Finalmente, queremos remarcar que HD 80077 es un objeto muy masivo de pos-secuencia principal y, por lo tanto, se espera que tenga una abundancia mayor de He. Sin embargo, no observamos ninguna contribución apreciable de las componentes prohibidas de He, dado que el perfil de línea He I $\lambda 4471$ Å ajusta muy bien considerando una abundancia solar (ver cap. 5). 


\section{HD 92964 (B2.5Ia)}

Lefèvre et al. (2009) miden para este objeto variaciones de luz con períodos de 2.119 días y 14.706 días. Estos autores también advierten asimetrías en las líneas de $\mathrm{H}_{\beta}$ y $\mathrm{H}_{\gamma}$ y atribuyen esta característica a los fuertes vientos que afectan a las líneas fotosféricas. También reparan en el hecho de que para modelar la línea de He I $\lambda 6678 \AA$ se requiere de una $\mathrm{v}_{\mathrm{mac}}$ elevada cuyo valor es el doble que el observado en las líneas de Si.

Los parámetros estelares derivados de la SED son $\mathrm{T}_{\text {ef }}=18000 \mathrm{~K}, \log g=2.19$ dex, $E(B-V)=0.481 \mathrm{mag}, \mathrm{R}_{\star}=76 \mathrm{R}_{\odot} \mathrm{y} \mathrm{d}=1806 \mathrm{pc}$ (cercano al valor de HIPPARCOS de $1851 \mathrm{pc}$ ). Usando la curva de extinción de van Breda et al. 1974 quienes encontraron que el cociente entre el índice de color y la extinción interestelar es 3.5 (este parámetro suele anotarse con la letra R). De la distancia medida por HIPPARCOS calculamos el coeficiente de absorción $\left(A_{V}=1.68 \mathrm{mag}\right)$ y obtenemos $M_{\text {bol }}=-9.14$ mag y $R_{\star}=62 R_{\odot}$, mientras que del diámetro angular (0.37 mas, Pasinetti Fracassini et al. 2001) el radio estelar derivado tiene un valor de $73 \mathrm{R}_{\odot}$. Como valor medio usamos $\mathrm{R}_{\star}=70 \mathrm{R}_{\odot}$.

A partir de nuestro modelado con FASTWIND determinamos los valores de $\mathrm{T}_{\text {ef }}=$ $18000 \mathrm{~K}$ y $\log g=2.2$ dex, que son similares a los valores que obtuvimos de la SED. La temperatura encontrada concuerda con los valores adoptados por Lefever et al. 2007) y Krtička \& Kubát 2001a), pero presenta grandes diferencias con los parámetros fotosféricos derivados por Fraser et al. 2010). Para modelar el espectro hemos usado una velocidad de rotación proyectada de $40 \mathrm{~km} / \mathrm{s}$ que es apenas más grande que la que se encuentra en la literatura $(28 \mathrm{~km} / \mathrm{s}$ y $31 \mathrm{~km} / \mathrm{s})$. Al igual que Lefever et al. 2007 encontramos un alto valor para $\mathrm{v}_{\mathrm{mac}}$.

Observaciones previas revelan una importante variación en la intensidad de las componentes en absorción y en emisión del perfil P Cygni de $\mathrm{H} \alpha$ (ver fig. A.1 dada en Lefever et al. 2007). Comparando nuestra observación de H $\alpha$ en 2013 con la presentada por Lefever et al., vemos que nuestro perfil muestra una mayor intensidad en sus dos componentes y logramos reproducir sólo la componente en emisión. Sin embargo derivamos un valor para $\mathrm{v}_{\infty}=370 \mathrm{~km} / \mathrm{s}$ que es cercano al medido en el rango UV $(435 \mathrm{~km} / \mathrm{s}$, Prinja et al. 1990a), y menor que los valores listados en la tabla 6.1. La tasa de pérdida de masa obtenida en nuestro trabajo es consistente con el valor determinado por Lefever et al. (2007).

\section{HD 99953 (B1/2Iab/b)}

Este es uno de los objetos menos estudiados de la muestra. Fraser et al. 2010 derivaron $\mathrm{T}_{\text {ef }}$ $=16800 \mathrm{~K}$ y $\log g=2.15$ dex. Basándonos en nuestro modelado de $\mathrm{H}_{\gamma}$, Si y He, obtuvimos: $\mathrm{T}_{\text {ef }}=19000 \mathrm{~K}$ y $\log g=2.30$ dex. Estos valores son compatibles con la asignación espectral del objeto y la SED observada ( $\mathrm{T}_{\mathrm{ef}}=18830 \mathrm{~K}, \log g=2.30 \mathrm{dex}, E(B-V)=0.56 \mathrm{mag}, \mathrm{R}_{\star}$ $=28 \mathrm{R}_{\odot}$ ) para $\mathrm{d}=1077 \mathrm{pc}$ (que es similar al valor de $1075 \mathrm{pc}$ medido por HIPPARCOS). Esta distancia y $E(B-V)$ predicen $\mathrm{M}_{\text {bol }}=-7.16$ mag y $\mathrm{R}_{\star}=22 \mathrm{R}_{\odot}$. Por lo tanto, adoptamos $\mathrm{R}_{\star}=25 \mathrm{R}_{\odot}$.

La línea de $\mathrm{H}_{\alpha}$ muestra un perfil $\mathrm{P}$ Cygni y su intensidad varía con el tiempo (ver fig. 3.2. La tasa de pérdida de masa y la velocidad terminal cambian en un factor $\sim 2.8 \mathrm{y}$ necesitamos diferentes valores de $\mathrm{v}_{\text {mac }}$ para modelar las diferentes épocas. 


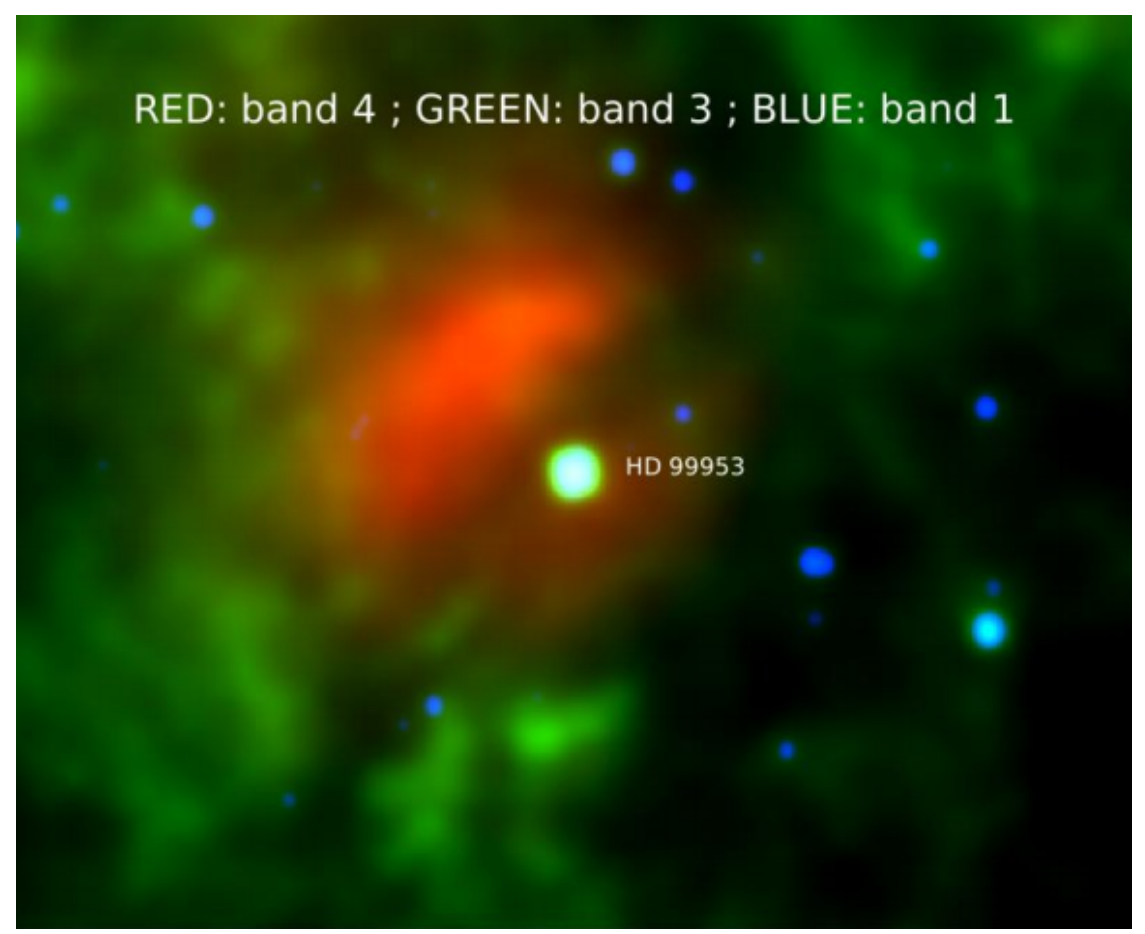

Figura 3.5. Imagen combinada de WISE en sus bandas W1 (azul), W3 (verde) y W4 (rojo) de HD 99953 que muestra la interacción del viento con el medio interestelar circundante

Este trabajo reporta por primera vez los valores de $\dot{\mathrm{M}}=0.08 \times 10^{-6} \mathrm{M}_{\odot} /$ año, $0.13 \times 10^{-6}$ $\mathrm{M}_{\odot} /$ año, y $0.22 \times 10^{-6} \mathrm{M}_{\odot} /$ año. La velocidad terminal va desde $250 \mathrm{~km} / \mathrm{s}$ a $700 \mathrm{~km} / \mathrm{s}$. Este amplio rango incluye el valor de $\mathrm{v}_{\infty}$ medido en las líneas UV $(510 \mathrm{~km} / \mathrm{s}$, Prinja et al. 1990a). La imagen combinada de WISE en sus bandas W1, W3 y W4 muestra emisiones de polvo calentado por la radiación proveniente de la estrella (ver fig. 3.5

\section{HD 111973 (B2/3Ia)}

Esta binaria espectroscópica fue clasificada como B3I (Chini et al. 2012). Presenta variaciones de luz con un período de 57.11 días y 9.536 días KKoen \& Eyer 2002.

La SED fue ajustada con un modelo TLUSTY con $\mathrm{T}_{\text {ef }}=17180 \mathrm{~K}, \log g=2.18$ dex, $\mathrm{R}_{\star}$ $=48.6 \mathrm{R}_{\odot}, E(B-V)=0.38 \mathrm{mag}$ y d $=1660 \mathrm{pc}$. El exceso de color fue obtenido usando la escala de calibración de Flower 1996). Con la distancia y el exceso de color derivados, obtuvimos una $\mathrm{M}_{\mathrm{bol}}=-7.81 \mathrm{mag}$ y $\mathrm{R}_{\star}=40 \mathrm{R}_{\odot}$. Por otro lado, cuando se tiene en cuenta el tamaño angular (0.26 mas) obtenemos $\mathrm{R}_{\star}=46 \mathrm{R}_{\odot}$, el cual está más cerca del derivado por Pasinetti Fracassini et al. 2001, $47 \mathrm{R}_{\odot}$ ). Adoptamos, por lo tanto, $\mathrm{R}_{\star}=46 \mathrm{R}_{\odot}$.

Pudimos ajustar todas las líneas fotosféricas y los valores de $\mathrm{T}_{\text {ef }} \mathrm{y} \log g$ (16500 K y 2.1 dex) derivados son similares a aquellos reportados por Fraser et al. 2010 $16000 \mathrm{~K}$ y 2.3 dex) y Prinja \& Massa 2010a, $16500 \mathrm{~K}$ ).

La línea $\mathrm{H}_{\alpha}$ muestra variaciones temporales cortas: observamos un perfil $\mathrm{P}$ Cygni con una componente en emisión débil que cambia a un perfil de absorción la siguiente noche 
(ver fig. 3.2. Esta variación es similar a la escala dinámica tempora ${ }^{4}$ esperada en un viento típico de una estrella BSG ( 1.3 días $)$.

Modelamos $\mathrm{H}_{\alpha}$ y derivamos una velocidad terminal menor que la observada en el UV $(520 \mathrm{~km} / \mathrm{s}$ Prinja \& Massa 2010a). Más aún, fue necesario modelar este perfil con un alto valor de $\mathrm{v}_{\text {mac }}(160-190 \mathrm{~km} / \mathrm{s})$.

Este trabajo reporta por primera vez el valor de la tasa de pérdida de masa que varía entre $0.14 \times 10^{-6} \mathrm{M}_{\odot} /$ año y $0.21 \times 10^{-6} \mathrm{M}_{\odot} /$ año.

\section{HD 115842 (B0.5Ia/ab)}

Koen \& Eyer 2002 reportaron variaciones fotométricas en escala de 13.38 días y variaciones en el perfil de la línea $\mathrm{H}_{\alpha}$. Nuestro espectro despliega una línea $\mathrm{H}_{\alpha}$ en emisión pura (ver fig. 3.2 mientras que el observado por Crowther et al. 2006a muestra un perfil $\mathrm{P}$ Cygni. $\mathrm{H}_{\beta}$ se ve en absorción pura y el perfil es ligeramente asimétrico (ver fig. 6).

De la SED derivamos $\mathrm{T}_{\mathrm{ef}}=25830 \mathrm{~K}, \log g=2.73 \mathrm{dex}, \mathrm{R}_{\star}=38 \mathrm{R}_{\odot}, E(B-V)=0.6$ mag, y d $=1543$ pc. Este exceso de color ajusta la depresión observada en los $2200 \AA$ y es mayor que el valor $E(B-V)=0.53 \mathrm{mag}$ derivado por Flower (1996). La distancia obtenida es consistente con las paralajes de HIPPARCOS y GAIA (0.65 mas y 0.6105 mas, respectivamente) y con la distancia de 1583 pc estimada a partir de las líneas de Ca II, $\mathrm{H}$ y $\mathrm{K}$ (Megier et al. 2009). Usando la distancia de HIPPARCOS y $E(B-V)=0.6 \mathrm{mag}$ calculamos $\mathrm{M}_{\text {bol }}=-9.22$ mag y $R_{\star}=32 \mathrm{R}_{\odot}$, mientras que del tamaño angular (0.22 mas, Pasinetti Fracassini et al. 2001 derivamos $R_{\star}=33 \mathrm{R}_{\odot}$. Por lo tanto, adoptamos $\mathrm{R}_{\star}=35$ $\mathrm{R}_{\odot}$.

Logramos muy buenos ajustes de las líneas fotosféricas con $\mathrm{T}_{\text {ef }}=25500 \mathrm{~K}$ y $\log g$ $=2.75$ dex. Los parámetros estelares derivados en este trabajo concuerdan con aquellos encontrados por la SED (ver tabla 3.1. y por Crowther et al. 2006a y Fraser et al. 2010 (ver tabla 6.1). La tasa de pérdida de masa que calculamos $\left(1.8 \times 10^{-6} \mathrm{M}_{\odot} /\right.$ año) es menor, y la velocidad terminal $(1700 \mathrm{~km} / \mathrm{s})$ es mayor que las estimaciones realizadas por Crowther et al. 2006a $\left(2.0 \times 10^{-6} \mathrm{M}_{\odot} /\right.$ año, $\left.1180 \mathrm{~km} / \mathrm{s}\right)$. Nuestro valor para $\mathrm{v}_{\infty}$ es incluso mayor que el medido con el satélite IUE $(1125 \mathrm{~km} / \mathrm{s})$ por Evans et al. 2004). Estos últimos autores reportaron un valor muy alto para la velocidad de macroturbulencia $(225 \mathrm{~km} / \mathrm{s})$ que es el doble del valor que nosotros obtuvimos.

La imagen de WISE en sus bandas W3 y W4 muestran un arco de choque muy amplio y definido (ver fig. 3.6 que estaría relacionado con una fuerte interacción entre el viento de la estrella y el medio interestelar circundante. Adicionalmente, una estructura de densidad asimétrica parece estar presente en la banda W1 con un tamaño angular mayor que la resolución del satélite para esa banda.

\section{HD 148688 (B1Iaeqp)}

Los datos de HIPPARCOS correlacionan con los períodos de variación de luz de 1.845 días

\footnotetext{
${ }^{4} \tau_{d y n} \simeq \sqrt{R_{\star}^{3} /(G M)}$, es el tiempo necesario para que la estrella vuelva a su estado de equilibrio, es decir, cuando el balance entre las fuerzas de presión y de gravedad sea distribuido por algún proceso dinámico (ver detalles en Aerts et al. 2010a.
} 


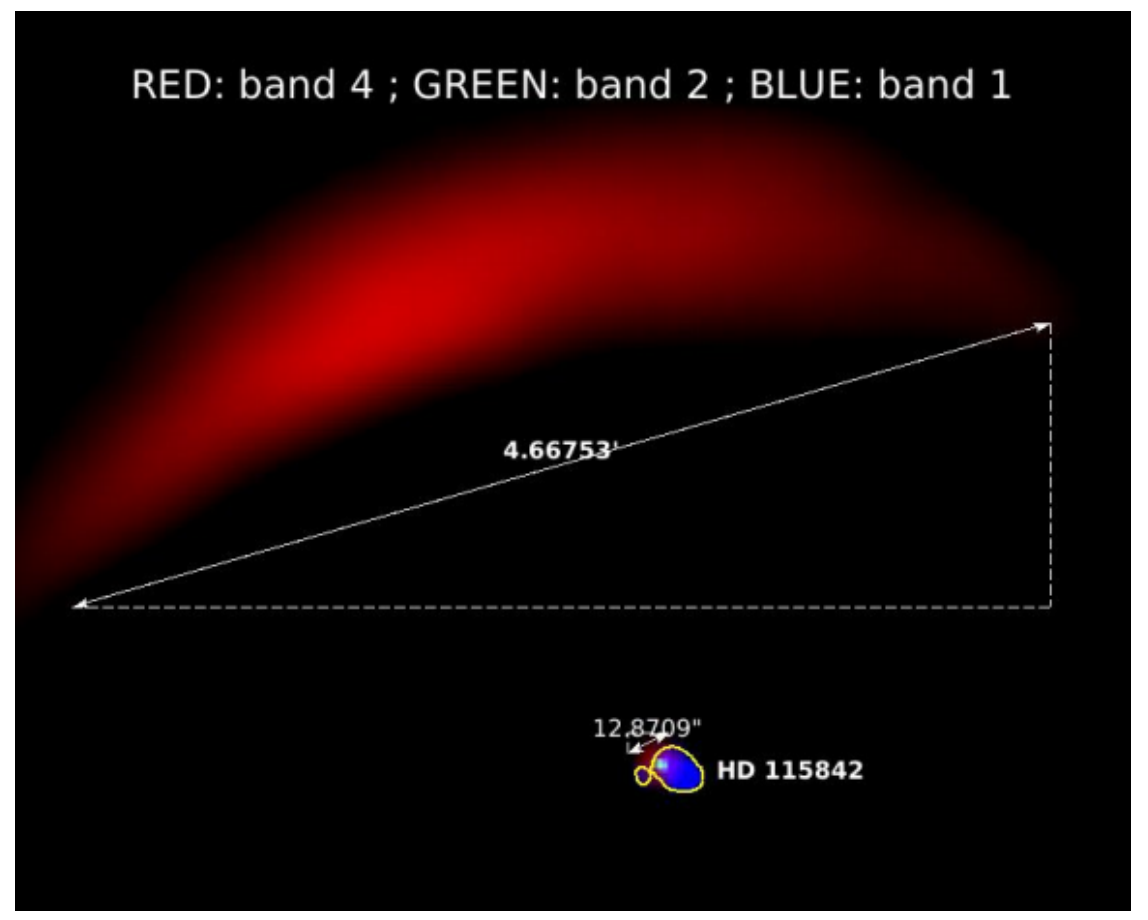

Figura 3.6. Imagen del satélite WISE donde pueden verse estructuras de arco de choque y de densidad asimétrica asociada a HD 115842. Las curvas amarillas representan regiones con el mismo brillo, cada una asociada con un lóbulo diferente. 
y 6.329 días (Lefever et al.2007.

El mejor modelo de ajuste a la SED arroja una $\mathrm{T}_{\text {ef }}=20650 \mathrm{~K}, \log g=2.2 \operatorname{dex}, \mathrm{R}_{\star}=$ $34 \mathrm{R}_{\odot}$, y una $\mathrm{d}=838 \mathrm{pc}$ (mientras que la distancia de HIPPARCOS es $833 \mathrm{pc}$ ). Esto da una $\mathrm{M}_{\mathrm{bol}}=-7.97$ mag y un $\mathrm{R}_{\star}=26 \mathrm{R}_{\odot}$, para un $E(B-V)=0.54$ mag. Este exceso se obtuvo de datos extraídos de Flower 1996 y también ajusta la depresión de los $2200 \AA$ presente en la SED. Hemos adoptado un $\mathrm{R}_{\star}=31 \mathrm{R}_{\odot}$.

Los parámetros estelares derivados con FASTWIND ( $\mathrm{T}_{\mathrm{ef}}=21000 \mathrm{~K}$ y $\log g=2.45 \mathrm{dex}$ ) están de acuerdo con los publicados en los trabajos de Fraser et al. 2010 y Lefever et al. 2007 .

La línea de $\mathrm{H}_{\alpha}$ de la fig. 3.2 muestra un perfil $\mathrm{P}$ Cygni que parece ser similar al reportado por Lefever et al. 2007), mientras que el espectro de Crowther et al. 2006a exhibe una línea de emisión.

Nuestro modelado logra reproducir sólo la componente en emisión del perfil P Cygni, obteniendo como resultado un valor para la tasa de pérdida de masa que es menor en un factor 1.1 y 1.4 (ver tabla 6.1) que los valores encontrados por Lefever et al. (2007) y Crowther et al. 2006a), respectivamente. La velocidad terminal que derivamos (1200 $\mathrm{km} / \mathrm{s}$ ) es igual a la encontrada por Lefever et al. (2007) aunque es mayor que las mediciones derivadas de las líneas UV (725 km/s, Prinja et al. 1990a).

\subsubsection{Comentarios globales de las propiedades estelares y del viento}

En este capítulo se han analizado las propiedades estelares y del viento de 19 estrellas BSGs mediante el ajuste de perfiles sintéticos a los observados. Para tres de estos objetos se determinaron por primera vez los parámetros del viento y para las estrellas con más de una observación hemos encontrado variaciones en las condiciones del viento. Aunque es sabido que las estrellas presentan variaciones espectrales, es posible describir sus propiedades globales, y a continuación pasaremos a detallarlas.

La fig. 3.7 muestra la relación entre la $\mathrm{T}_{\text {ef }}$ de las BSGs y el subtipo espectral. La muestra de esta figura es una colección de datos de la literatura y este trabajo. Con la excepción de aquellas estrellas cuya $T_{\text {ef }}$ fue determinada con el método BCD (Zorec et al. 2009a), la determinación de este parámetro estelar fue obtenido mediante el ajuste de perfiles de las líneas de HeI, HeII y de la intensidad del cociente Si IV/Si III o Si III/Si II, como se ha hecho en el presente estudio. Realizamos un ajuste a la relación entre $\mathrm{T}_{\text {ef }}$ observada vs el subtipo espectral usando un polinomio de grado tres $(\mathrm{a}=-54.6 \pm 7.7, \mathrm{~b}=973.4 \pm$ $107.5, \mathrm{c}=-6054.3 \pm 387.9 \mathrm{y} \mathrm{d}=27316 \pm 331)$. Los coeficientes derivados concuerdan muy bien con los coeficientes $(-45.9,878,-6000,27800)$ obtenidos por Markova \& Puls 2008. Nuestro ajuste (por mínimos cuadrados) se ilustra en la fig. 3.7 mediante una curva a trazos, mientras que la banda gris está asociada con una dispersión de $1290 \mathrm{~K}$. Las supergigantes de nuestra muestra, con las excepciones de HD 52089, HD 53138 y HD 47240 (indicadas en la figura con un círculo abierto), caen dentro de la relación trazada. Más aún, podemos observar que los apartamientos de la temperatura efectiva de las BSGs de tipo espectral 


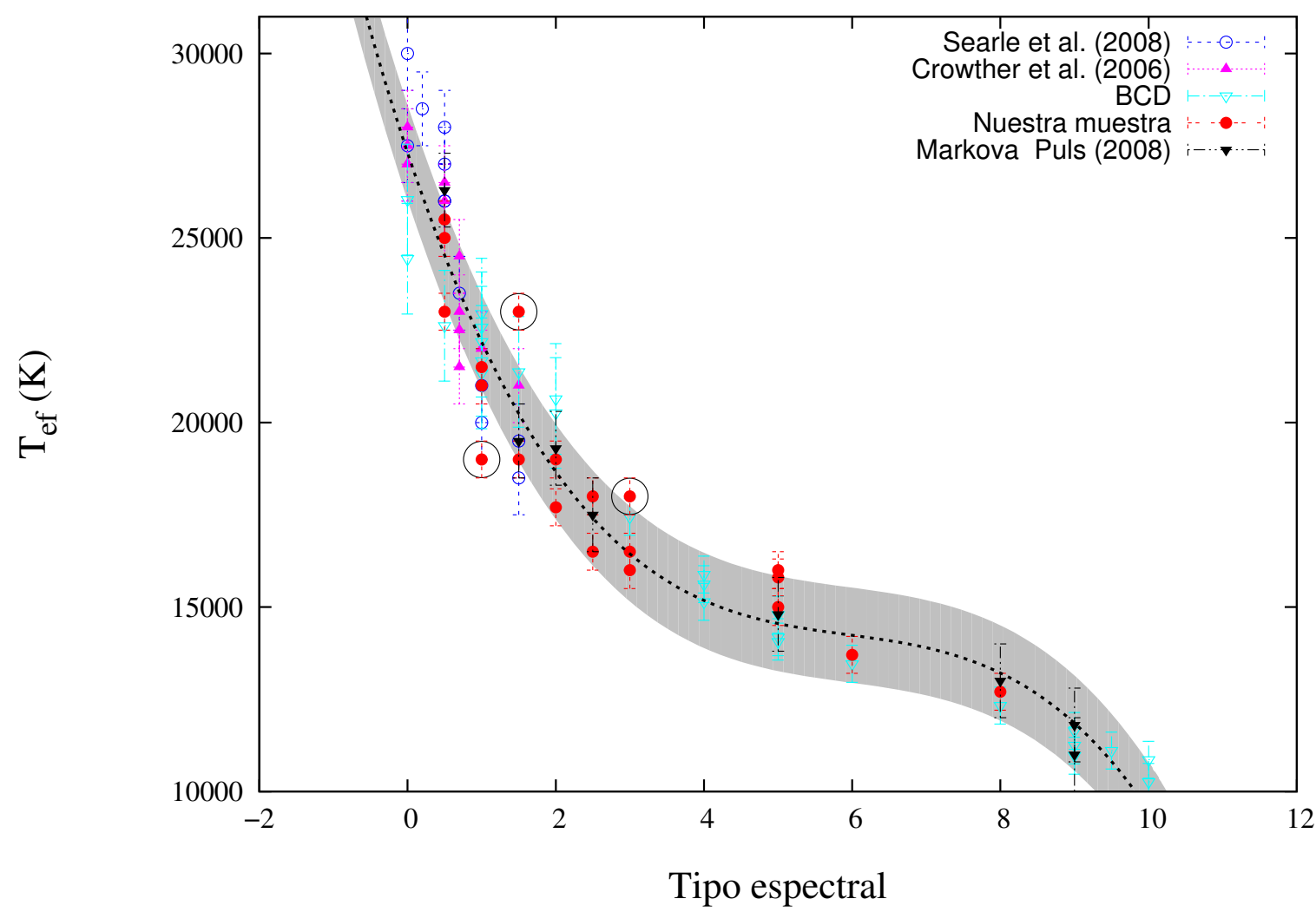

Figura 3.7. Temperatura efectiva en función del subtipo espectral. La relación fue ajustada con un polinomio de grado tres (línea negra a puntos) con una dispersión de $1290 \mathrm{~K}$ (banda gris). Comparación de nuestra muestra de BSGs galácticas con un conjunto de BSGs encontradas en la literatura. Los puntos de nuestra muestra marcados con un círculo abierto corresponden a objetos que no siguen la relación.

temprano obtenida por diferentes autores van de $0 \mathrm{~K}$ a $5000 \mathrm{~K}$ y tienden a ser menores para los subtipos tardíos. Esta misma tendencia también fue observada en el trabajo de Markova et al. 2008a).

La fig. 3.8 despliega la relación lineal entre $\log \mathrm{T}_{\text {ef }} \mathrm{y} \log \mathrm{g}_{\mathrm{ef}}$ (gravedad superficial corregida por la aceleración centrífuga, ver tabla 6.1. Este tipo de relación fue previamente reportada por Searle et al. 2008a). Ajustamos una relación lineal cuya pendiente es 3.98 \pm 0.31 . Esto indica que la mayoría de las estrellas de nuestra muestra tienen una relación $\mathrm{L} / \mathrm{M}$ similar $(\log (\mathrm{L} / \mathrm{M}) \sim 4)$, como es esperable, ya que $\mathrm{L} \propto \mathrm{T}_{\mathrm{ef}}^{4}$. Sin embargo, observamos que aquellas estrellas que tienen un alto valor de $\log g_{\text {ef }}(\geq 2.5$ dex $)$, en particular, HD 42087, HD 52089 y HD 64760 no siguen dicha relación. Dos de estas estrellas, HD 42087 y HD 64760, están muy cerca de la TAMS (Terminal Age Main Secuence) por lo que podríamos pensar que recién abandonaron la secuencia principal. Particularmente HD 64760 es un alto rotador y, en consecuencia, deberíamos observar una luminosidad bolométrica 


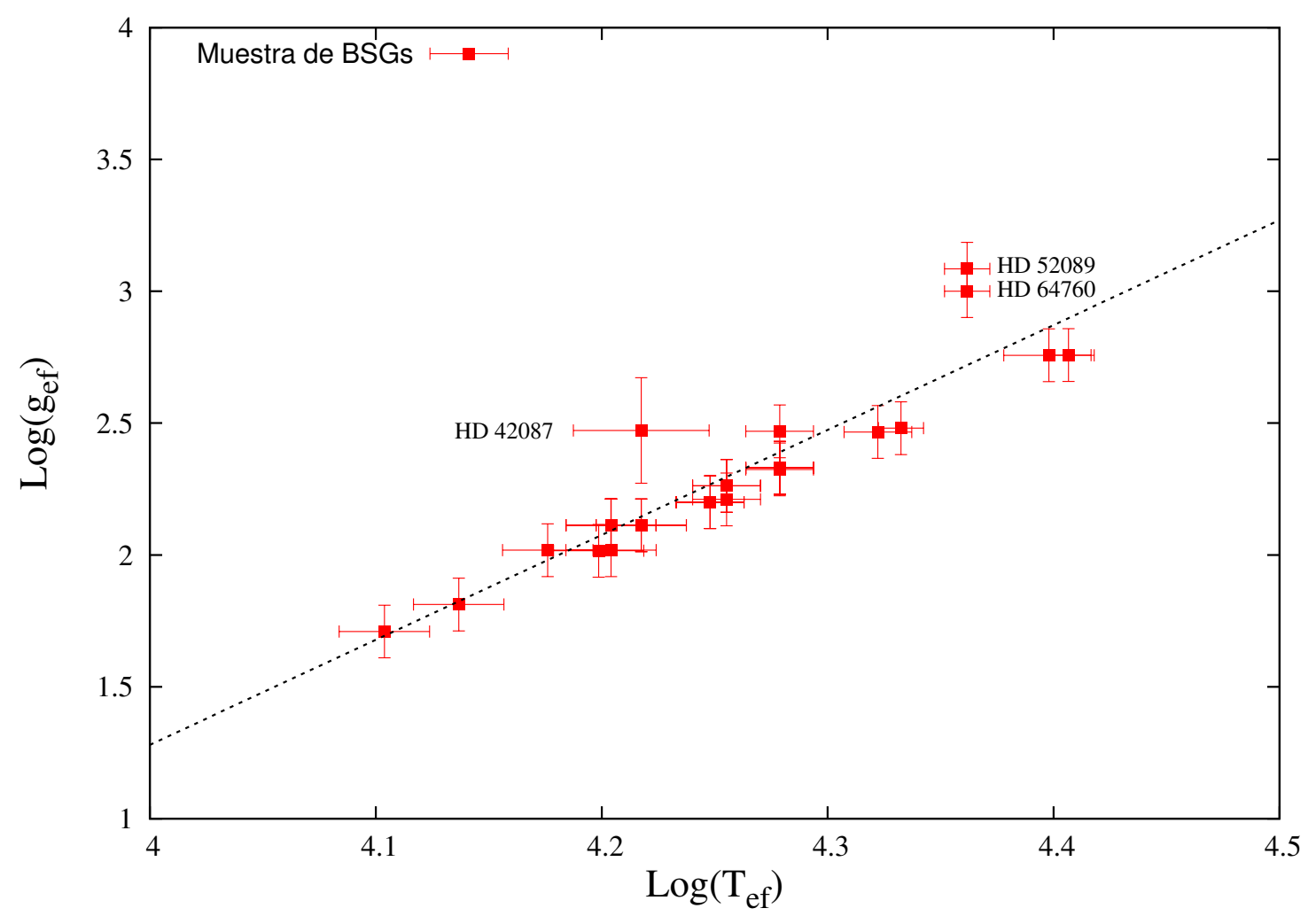

Figura 3.8. Relación lineal $\log T_{\text {ef }}-\log g_{\text {ef }}$ de nuestra muestra de BSGs galácticas (línea punteada). La gravedad superficial fue corregida por la aceleración centrífuga.

aparente mayor, y una temperatura efectiva menor, que aquella esperada para sus contrapartes no rotantes (Frémat et al. 2005, Zorec et al. 2005), fingiendo una etapa evolutiva posterior.

También calculamos los valores promedios para la tasa de momento mecánico total del viento, $\mathrm{D}_{\mathrm{mom}}$, en función de la luminosidad de la estrella, es decir, la relación momento luminosidad modificada (ver secc. 2.5.2) dada por

$$
D_{\text {mom }}=\dot{M} v_{\infty} R^{0,5} \propto L^{1 / \alpha_{\text {ef }}},
$$

donde $\alpha_{\text {ef }}=\alpha-\delta$ (ver detalles en Kudritzki et al. 1999; Puls et al. 1996); $\alpha$ y $\delta$ son los parámetros de la fuerza de radiación relacionados con la opacidad de la línea y la ionización del viento, respectivamente.

Nuestra muestra se divide en dos grupos diferentes (ver fig. 3.9): las estrellas BSGs tempranas (B0-B1.5, diamantes azules) por un lado, y las de tipo espectral medio y tardío (B2B3, diamantes verdes y B4-B9, diamantes rojos, respectivamente). Estos dos grupos parecen estar claramente separados, siguiendo dos relaciones diferentes. Sin embargo, encontramos entre las BSGs tempranas, tres BSGs de tipo espectral medio (HD 41117, HD 42087 y 
HD 99 953) con tasas de momento del viento comparables a las estrellas tempranas. Entre las relaciones lineales encontradas, observamos que las estrellas más tempranas de nuestra muestra de BSGs más esas tres estrellas de tipo espectral mediano (caracterizadas por un círculo en la fig. 3.9 pueden ser ajustadas con la siguiente relación:

$$
\log D_{\text {mom }}=1,96( \pm 0,28) \log L / L_{\odot}+17,98( \pm 1,43), \text { para B0 }-\mathrm{B} 1,5
$$

La segunda relación momento-luminosidad fue trazada usando las estrellas BSGs con tipos espectrales entre B2 y B9 (en triángulos en la fig. 3.9). Los valores para el momento del viento para estas estrellas son claramente menores que los que presentan las estrellas de temprano tipo espectral y la relación despliega una pendiente diferente. Para estos objetos encontramos que el ajuste nos arroja el siguiente resultado:

$$
\log D_{\text {mom }}=1,43( \pm 0,42) \log L / L_{\odot}+19,94( \pm 2,23), \text { para B2 - B9 }
$$

Esta observación muestra que la relación momento luminosidad es mayor para las estrellas de tipo espectral temprano que para las de tipo espectral medio/tardío. Más allá de los errores involucrados, esta tendencia es opuesta a la observada por Kudritzki et al. (1999) y a la predicha por Vink 2000. Este último autor obtiene las siguientes relaciones teóricas:

$$
\log D_{\text {mom }}=1,826( \pm 0,044) \log L / L_{\odot}+18,68( \pm 0,26), \mathbf{T}_{\text {ef }}>\mathbf{2 7 0 0 0} \mathbf{K}
$$

$$
\log D_{\text {mom }}=1,914( \pm 0,043) \log L / L_{\odot}+18,52( \pm 0,26), \mathbf{1 2 0 0 0} \mathbf{K}<\mathbf{T}_{\text {ef }}<\mathbf{2 2 0 0 0 ~ K}
$$

las cuales implican que la relación momento-luminosidad modificada es mayor para las estrellas supergigantes más frías que para las más calientes y que $\alpha_{\mathrm{ef}}=0.522$ y 0.548 , respectivamente. Si bien la relación predicha para $\mathrm{T}_{\text {ef }}>27000 \mathrm{~K}$ concuerda con las observaciones de estrellas de tipos espectrales mayores que B1, para la segunda relación existe una inconsistencia entre los valores de la tasa de pérdida de masa medidos para las estrellas B por diferentes autores (ver Crowther et al. 2006a Searle et al. 2008a Markova \& Puls 2008 .

En la fig. 3.9. las observaciones de un mismo objeto están conectadas con una línea para resaltar los efectos de la variabilidad. Para estas estrellas se consideraron valores medios de $\log D_{\text {mom }}$ (caracterizados por puntos negros en la fig. 3.9 para calcular el ajuste lineal de cada grupo.

Es interesante remarcar que el régimen del viento que describe cada relación momentoluminosidad es diferente. De la tabla 3.3 encontramos que las estrellas de tipo espectral temprano tienen en su mayoría $\beta \lesssim 2$ y velocidades terminales mayores a $500 \mathrm{~km} / \mathrm{s}$, mientras que las de tipos espectrales medios y tardíos tienen $\beta \geq 2$ y velocidades terminales menores a $500 \mathrm{~km} / \mathrm{s}$. Por otro lado, el parámetro $\alpha_{\text {ef }}$, que es la inversa de la pendiente de la relación momento-luminosidad, cambia de 0.5 para las BSGs tempranas a 0.7 para las 


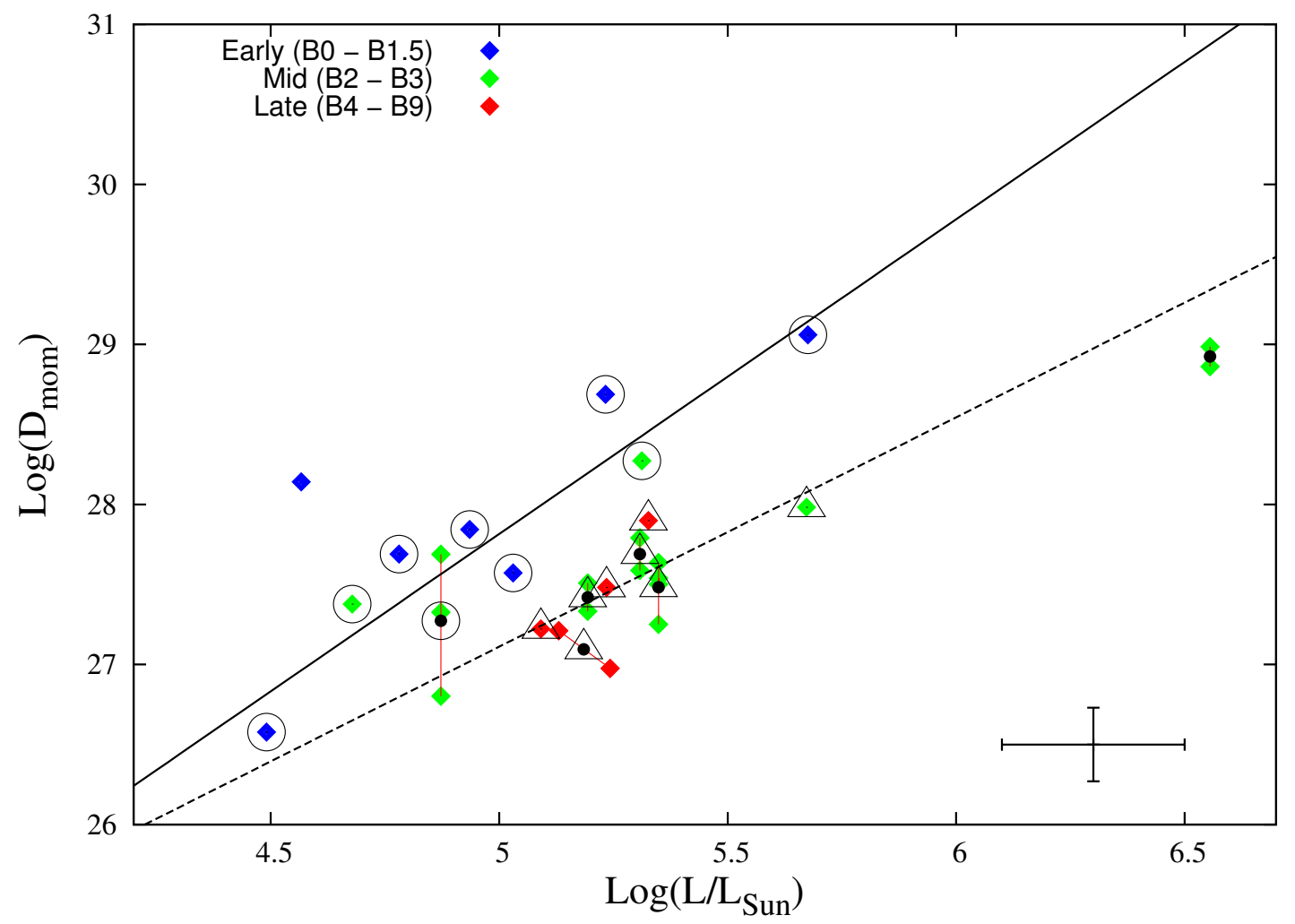

Figura 3.9. Relaciones momento-luminosidad observadas. Los diamantes azules, verdes y rojos representan a las estrellas BSGs de tipo espectral temprano (Early), medio (Mid) y tardío (Late), respectivamente. El ajuste superior fue derivado usando los puntos marcados con círculos mientras que para determinar la relación inferior se utilizaron los datos marcados con triángulos. Los diamantes conectados con líneas corresponden a observaciones de un mismo objeto en diferentes épocas (los puntos negros representan sus valores medios). 
tardías. Bajo esta condición, diferentes mecanismos (por ejemplo pulsaciones, clumping, etc.) podrían contribuir a impulsar el viento.

Finalmente, la fig. 3.10 muestra el cociente $\mathrm{v}_{\infty} / \mathrm{v}_{\text {esc }}$ como función de $\log \mathrm{T}_{\text {ef }}$. En esta figura presentamos nuestros resultados en conjunto con dos regiones de temperatura definidas por Markova \& Puls 2008, que muestran diferentes cocientes $\mathrm{v}_{\infty} / \mathrm{v}_{\text {esc }}: 3.3$ para temperaturas efectivas por encima de $\operatorname{los} 23000 \mathrm{~K}$, y 1.3 para las que están por debajo de $18000 \mathrm{~K}$ (ver cap. 2. secc. 2.9.1). Para poder comparar nuestros resultados con los de otros autores, en la fig. 3.10 están marcadas las regiones sombreadas que indican el ancho de los errores de $\mathrm{v}_{\infty} / \mathrm{v}_{\mathrm{esc}}$ del $33 \%$ para las más frías y del $43 \%$ para las más calientes estimados por Markova \& Puls 2008). Estos autores encuentran que $\Delta\left(\mathrm{v}_{\infty} / \mathrm{v}_{\text {esc }}\right) \simeq 0.7$ y 0.4 para $\mathrm{T}_{\text {ef }}>23 \mathrm{kK} \mathrm{y} \mathrm{T}_{\text {ef }}<18 \mathrm{kK}$, respectivamente, aunque el último valor puede estar subestimado ya que adoptaron $\mathrm{v}_{\infty}=\mathrm{v}_{\text {esc }}$ para algunas estrellas debido a la falta de diagnósticos.

La región intermedia, delimitada por líneas verticales negras, es la región correspondiente al salto de biestabilidad. Markova \& Puls 2008 mostraron que las estrellas localizadas en esta región despliegan un decrecimiento gradual en $\mathrm{v}_{\infty} / \mathrm{v}_{\text {esc }}$. En general, nuestros resultados muestran un comportamiento similar que los reportados por estos autores.

En particular, encontramos que varias de las estrellas de nuestra muestra están localizadas dentro de la región de biestabilidad. Como algunas son variables, también dibujamos en la fig. 3.10 las diferentes posiciones que ocuparían estos objetos durante diferentes épocas. Estas observaciones están conectadas con líneas negras.

La variación de $\mathrm{v}_{\infty} / \mathrm{v}_{\text {esc }}$ para las estrellas localizadas del lado frío del salto de biestabilidad parecen ser pequeñas. Pero HD 99953 (diamantes verdes con triángulos), que está dentro de la región, presenta una variación en el viento de manera tal que parecería cambiar de un régimen lento a uno rápido. La misma variación importante se observa para esta estrella en la relación momento-luminosidad. Con la excepción de HD 80077, que tiene una tasa de pérdida de masa muy grande, no observamos que un decrecimiento en el cociente $\mathrm{v}_{\infty} / \mathrm{V}_{\text {esc }}$ vaya acompañado de un incremento en la pérdida de masa, como predice Vink 2000. En general, las BSGs medias y tardías presentan valores de $\dot{M}$ que son similares o menores que los encontrados para estas estrellas localizadas en el lado caliente del salto de biestabilidad.

Todas estas observaciones y relaciones serán tratadas nuevamente en el cap. 5 donde nuestros resultados se compararán con los obtenidos por otros autores y se discutirá el posible origen de las variaciones observadas en los vientos de estas estrellas. 


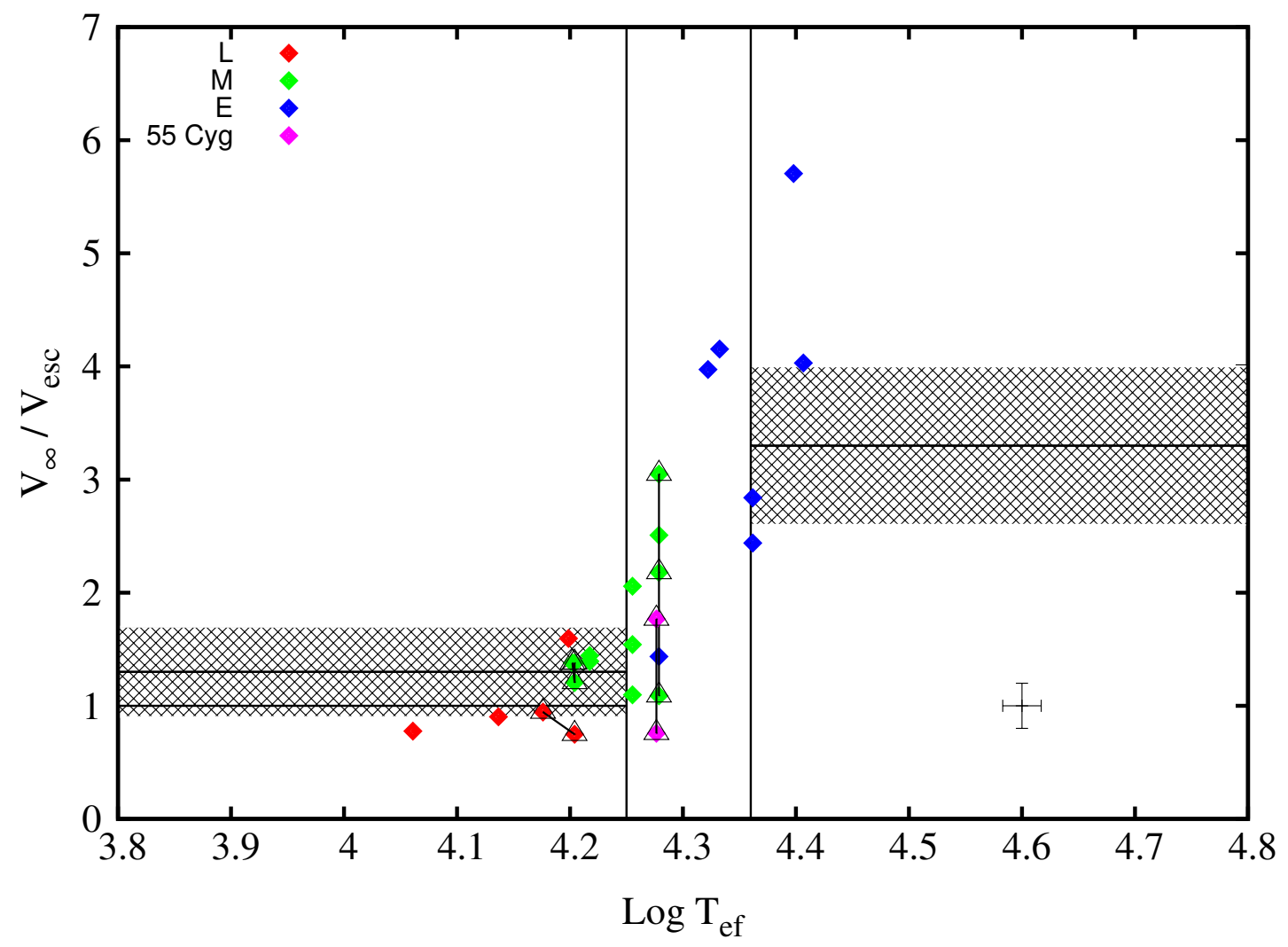

Figura 3.10. Cocientes de $v_{\infty} / v_{\text {esc }}$ en función de $\log T_{\text {ef }}$. Los tipos espectrales tempranos (E), medios (M) y tardíos (L) están indicados con diamantes azules, verdes y rojos, respectivamente. Estrellas con vientos variables están indicadas con triángulos y unidas por líneas. La mayor variabilidad es vista en HD 99953 que está localizada en la región limitada por las líneas verticales (región de biestabilidad). Las áreas sombreadas se refieren a dos zonas con diferentes $\mathrm{v}_{\infty} / \mathrm{v}_{\text {esc }}: 3.3$, para temperaturas efectivas por encima de $23000 \mathrm{~K}$, y 1.3 por debajo de $18000 \mathrm{~K}$. El ancho de las zonas sombreadas representa errores del $43 \%$ y $33 \%$ (ver texto). 


\section{Capítulo 4}

\section{El enigmático viento de 55 Cygni}

Este capítulo está dedicado enteramente al análisis de las propiedades del viento y de la fotosfera de 55 Cyg Para este objeto realizamos una campaña de observación de casi 5 años donde se han obtenido numerosos espectros y es por este motivo que dedicamos un capítulo exclusivamente a este objeto. El estudio realizado está basado en la misma metodología presentada en el capítulo anterior, es decir, mediante el ajuste de perfiles de líneas teóricos a los observados. El análisis de una gran cantidad de espectros nos permitió determinar de manera muy precisa los parámetros de esta estrella para poder discutirlos y compararlos con resultados previos.

El capítulo está estructurado en cuatro secciones. En las secs. 4.1 y 4.2 describiremos las características de la estrella encontradas en la literatura y las características asociadas con las observaciones, respectivamente. En la siguiente sec. 4.3 estarán plasmados nuestros resultados de las mediciones de los parámetros estelares y del viento, en conjunto con un análisis de la estimación de los errores, y en la sec. 4.4 discutiremos las propiedades del viento de 55 Cyg y su estado evolutivo.

\subsection{La estrella}

55 Cyg (HD 198478, MWC 353, HR 7977, HIP 102724, $\alpha=20 \mathrm{~h} 48 \mathrm{~m}$ 56s.291,$\delta=+46^{\circ}$ 06' 50".88, J2000.0) es el objeto más brillante de la asociación Cyg OB7 (Humphreys 1978 y fue clasificada como B3Ia por Morgan \& Roman 1950 y como B4Ia por Zorec et al. 2009a). El valor de la paralaje medido con HIPPA $\overline{R C O S}$ es de $1.40 \pm 0.17$ mas van Leeuwen 2007b ubicándola a unos $714 \pm 86$ pc. La extinción visual interestelar determinada es de 1.62 mag Barlow \& Cohen 1977, Humphreys 1978).

$\mathrm{Al}$ ser uno de los objetos más brillantes del hemisferio norte, varios estudios le fueron dedicados, revelándose dos hechos interesantes: diferentes elementos químicos despliegan diferentes velocidades radiales (Hutchings 1970), mientras que las mismas líneas muestran

\footnotetext{
${ }^{1}$ Los resultados fueron publicados en la revista Astronomy \& Astrophysics, titulado Interplay between pulsations and mass loss in the blue supergiant 55 Cygnus $=$ HD 198478, por Kraus, Haucke, Cidale et al. (2015) http://adsabs.harvard. edu/abs/2015A\%26A..581A. .75K
} 
fuertes variaciones en el tiempo. Por ejemplo, Underhill (1960) describe variaciones de velocidad radial de $25 \mathrm{~km} / \mathrm{s}$.

Observaciones fotométricas revelaron microvariaciones pero sus periodicidades fueron difíciles de establecer. Mientras que Rufener \& Bartholdi 1982 reportaron una variabilidad de 18 días en la banda V, Koen \& Eyer (2002) encontraron periodicidades de 4.88 días usando los datos del satélite HIPPARCOS. Por otro lado, no se reportaron períodos claros en los trabajos de Percy \& Welch 1983, van Genderen et al. (1989) y Lefèvre et al. 2009.

La línea de $\mathrm{H}_{\alpha}$ es altamente variable. Underhill 1960 y Rzaev 2012 remarcaron que $\mathrm{H}_{\alpha}$ mantenía un perfil $\mathrm{P}$ Cygni pero con una gran variabilidad en su intensidad. Ebbets (1982 notó que durante sus observaciones, $\mathrm{H}_{\alpha}$ mostró emisiones débiles en sus alas, que no fueron vistas en el espectro que adquirió dos meses después. Y más aún, Maharramov 2013 reportó que $\mathrm{H}_{\alpha}$ puede desaparecer por completo del espectro.

Se encuentra en la literatura que la velocidad de rotación proyectada y la velocidad terminal son también muy variables. Los valores para v sen i van desde $0 \mathrm{~km} \mathrm{~s}^{-1}$ Slettebak \& Howard 1955) a un valor máximo de $61 \mathrm{~km} / \mathrm{s}$ (Howarth et al. 1997b). Este último fue obtenido del espectro UV. Otras determinaciones basadas en el espectro visual utilizando diferentes técnicas incluyen $42 \mathrm{~km} / \mathrm{s}$ (Day \& Warner 1975), $49 \pm 1 \mathrm{~km} / \mathrm{s}$ Gies \& Lambert 1992a, $45 \pm 20 \mathrm{~km} / \mathrm{s}$ McErlean et al.|1999a), $35 \pm 9 \mathrm{~km} / \mathrm{s}$ Abt et al. 2002 y $39 \mathrm{~km} / \mathrm{s}$ (Markova et al. 2008a). En cuanto a la velocidad terminal, Prinja et al.|(1990b) obtuvieron $470 \mathrm{~km} / \mathrm{s}$ basándose en las líneas de resonancia de C IV, mientras que Prinja \& Massa $2010 \mathrm{~b}$ derivaron un valor mayor, de $560 \mathrm{~km} / \mathrm{s}$, a partir del ion de Si IV. Ambos valores son bajos si los comparamos con el valor de $690 \mathrm{~km} / \mathrm{s}$ obtenido de la teoría de vientos impulsados por radiación Krtička \& Kubát 2001b. Sin embargo, Markova et al. 2008a notaron que el valor observado de $470 \mathrm{~km} / \mathrm{s}$ es muy alto para dar un ajuste satisfactorio en la línea $\mathrm{H}_{\alpha}$ y que el modelo lograría un buen ajuste si la velocidad terminal tuviera el valor de sólo $\sim 200 \mathrm{~km} / \mathrm{s}$. Estos autores especularon que 55 Cyg podría tener un viento cuya estructura de velocidad fuera variable en el tiempo.

A la vista de aquellas variaciones en las cantidades observables descriptas anteriormente, no resulta sorprendente que haya cierta dispersión en los parámetros físicos derivados. Aunque la determinación de los parámetros fundamentales depende de la técnica que se use, se encuentra que hay diferentes valores de $\mathrm{T}_{\text {ef }}$ usando modelos construidos con las mismas aproximaciones. A partir de mediciones basadas en la discontinuidad de Balmer, el valor de $\mathrm{T}_{\text {ef }}$ determinado es de $16450 \mathrm{~K}$ Gies \& Lambert 1992a) y $15380 \pm 860 \mathrm{~K}$ Zorec et al.2009b. Usando la aproximación de capas plano paralelas hidrostáticas en NLTE para una atmósfera estelar y el balance de ionización del Si, las temperaturas derivadas tienen valores de $18000 \mathrm{~K}$ (McErlean et al. 1999a), $18500 \mathrm{~K}$ Monteverde et al. 2000 y $17000 \mathrm{~K}$ Jurkić et al. 2011), mientras que para un modelo de línea en NLTE (teniendo en cuenta el bloqueo y el blanketing del viento) se obtienen valores de $16500 \mathrm{~K}$ Crowther et al.2006b y 17500 K Searle et al. 2008b Markova et al. 2008a. En todos los casos la gravedad superficial determinada está alrededor de $\log \mathrm{g}=2.2 \pm 0.1$ dex.

Como la línea de $\mathrm{H}_{\alpha}$ es generalmente usada como un indicador para determinar la tasa de pérdida de masa (Puls et al. 2008), es esperable que ajustando la observación de dicha línea en diferentes épocas, resulte en diferentes estimaciones de $\dot{M}$. De hecho, los valores de 
este parámetro varían sustancialmente. Mientras que Searle et al. 2008b encontraron $\dot{M}$ $=5 \times 10^{-7} \mathrm{M}_{\odot} /$ año, el valor de Crowther et al. 2006b) es un factor dos menor, y aunque ambos trabajos usen el mismo valor para la velocidad terminal $(470 \mathrm{~km} / \mathrm{s})$, los valores para el radio estelar son de $46.1 \mathrm{R}_{\odot}$ y $40 \mathrm{R} \odot$. Adicionalmente, Markova et al. 2008a obtienen una gran incerteza en su determinación de $\dot{\mathrm{M}}=1.175-4.07 \times 10^{-7} \mathrm{M}_{\odot} /$ año debido la gran incerteza en la velocidad terminal $(200 \mathrm{~km} / \mathrm{s}$ vs $470 \mathrm{~km} / \mathrm{s})$. Cabe mencionar que todos estos estudios usan modelos sin clumping.

Lo que aún no se mencionó en este resumen es el posible estadío evolutivo de 55 Cyg. Underhill 1969 reportó que la línea observada de $\mathrm{H}_{\gamma}$ no es tan intensa y que ello se debería a que 55 Cyg es pobre en $\mathrm{H}$ y que, por lor tanto, es una estrella en la fase de postsupergigante roja. Conclusiones similares fueron alcanzadas por Lennon et al. (1993b), Gies \& Lambert 1992a), Crowther et al. 2006b), y Searle et al. (2008b) a partir de la abundancia de CNO procesado determinado del espectro óptico, en particular su fuerte enriquecimiento en N. Markova et al. 2008a adicionalmente concluyen que la estrella debería ser rica en He. Estos últimos autores también encuentran una masa espectroscópica un tanto baja de $\sim 11 \mathrm{M}_{\odot}$ comparada con la alta luminosidad de la estrella, que la ubica en un track evolutivo de $25 \mathrm{M}_{\odot}$. Esta discrepancia en la masa podría indicar que la estrella haya perdido grandes cantidades de material durante la fase de supergigante roja. Esta idea encuentra apoyo en el trabajo de van Genderen 1989, que catalogan a 55 Cyg como una variable $\alpha$ Cyg. Si esta clasificación es válida, entonces 55 Cyg debería presentar muchos modos de pulsación, incluidos los modos radiales extraños Saio et al.2013b.

\subsection{Observaciones}

55 Cyg fue monitoreada espectroscópicamente entre el 15 de agosto de 2009 y el 22 de octubre de 2013, obteniendo un total de 344 espectros distribuidos en 64 noches. Estas observaciones fueron adquiridas con un espectrógrafo Coudé adosado al telescopio Perek de $2 \mathrm{~m}$. en el Observatorio de Ondřejov, República Checa 22 Las características de estos instrumentos en conjunto con las cámaras CCD brindaron una resolución de $\sim 13000$ en la región de $\mathrm{H}_{\alpha}$.

Por otro lado, colaboradores de Estados Unidos también adquirieron un total de 41 espectros, entre el 17 de octubre de 2013 y el 7 de noviembre de 2013 en el Observatorio Winer de Arizona, usando el Telescopio Espectroscópico Poznan, con una resolución de 40000 .

Como primer resultado, mostramos que la línea de $\mathrm{H}_{\alpha}$ despliega variaciones en una escala de tiempo de aproximadamente un día tanto en intensidad como en forma, presentando diferentes tipos de perfiles: P Cygni, emisión pura, casi completa ausencia de picos y con dobles o múltiples picos en emisión (ver fig. 4.1.

\footnotetext{
${ }^{2}$ Las observaciones están enmarcadas en un programa de observación de la Dra. Michaela Kraus, quien nos brindó los espectros ya reducidos.
} 


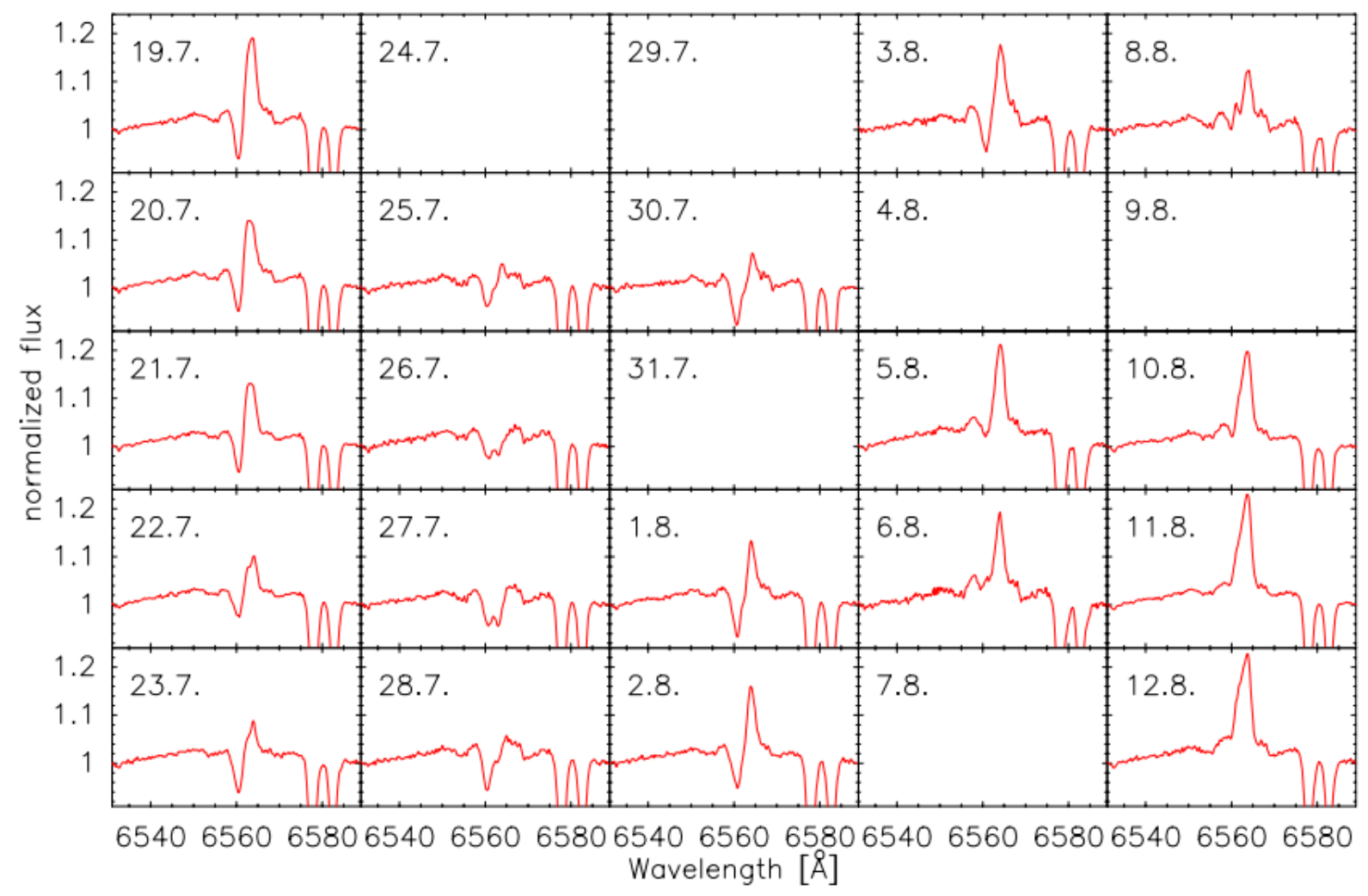

Figura 4.1. Variaciones noche a noche en la línea $\mathrm{H}_{\alpha}$ (flujo normalizado vs. longitud de onda), desde el 19/7 al 12/8/2013. El día y el mes están especificados en el extremo superior izquierdo de cada panel.

\subsection{Resultados}

\subsubsection{Modelado de las líneas}

Para cuantificar y analizar las variaciones espectrales de 55 Cyg ajustamos simultáneamente los perfiles observados de H, He y Si. El código usado en este trabajo también fue el FASTWIND.

Para obtener los perfiles de líneas sintéticos hemos iniciado los cálculos dando un conjunto de parámetros medios, tanto fotosféricos como los del viento, obtenidos de la literatura (ver sec. 4.1). Sin embargo, dado que esos parámetros no nos permitieron obtener buenos ajustes, ampliamos el rango de los parámetros para buscar el mejor ajuste mediante inspección visual. Para ello, tratamos a todos los parámetros $\left(\mathrm{T}_{\mathrm{ef}}, \log \mathrm{g}, \beta, \dot{\mathrm{M}}, \mathrm{v}_{\infty}, \mathrm{v}_{\mathrm{mac}}\right.$, $\mathrm{v}_{\text {rot }} \mathrm{y} \mathrm{R}_{\star}$ ) como parámetros libres y computamos una gran grilla de espectros sintéticos. Esto nos permitió encontrar modelos más próximos a nuestras observaciones, que una vez detectados fueron refinados para obtener los mejores ajustes.

Para refinar los parámetros estelares de la grilla utilizamos los espectros tomados en Arizona, ya que estos cubren los rangos espectrales que contienen a las líneas de HeI $\lambda 6678 \AA$, 
He I $\lambda 4713 \AA$ y He I $\lambda 4471 \AA$, Si II $\lambda 4128 \AA$ y Si II $\lambda 4130 \AA$ para poder estimar $\mathrm{T}_{\text {ef }}, \mathrm{y} \mathrm{H}_{\gamma}$ y $\mathrm{H}_{\delta}$ para medir $\log \mathrm{g}$ (ver cap. 2p. Desafortunadamente las observaciones de Ondřejov cubren un rango más renstringido de longitud de onda, brindando sólo He I $\lambda 6678 \AA$ A. Y aunque los espectros tienen menor resolución, estos presentan un mayor cociente $\mathrm{S} / \mathrm{N}$ que los espectros de Arizona.

El otro parámetro fundamental es el radio estelar, que debe ser determinado de una manera independiente. En la literatura los valores van de $38 \mathrm{R}_{\odot}$ Markova et al.2008a a 83 $\mathrm{R}_{\odot}$ Gies \& Lambert 1992b y el método más usado para su determinación es por lo general a partir del ajuste de la SED. Como las discrepancias son significativas, en este caso hemos usado el método BCD descripto en el cap. 2.4 Obtenemos una magnitud bolométrica de $-8.5 \pm 0.3 \mathrm{mag}$ que nos brinda un $\log \mathrm{L} / \mathrm{L}_{\odot}=5.3 \pm 0.1$ y un $\mathrm{R}_{\star}=61 \pm 8 \mathrm{R}_{\odot}$. Este valor del radio concuerda con el valor de $61 \mathrm{R}_{\odot}$ calculado por Pasinetti Fracassini et al. (2001) a través de mediciones de tamaño angular. Este parámetro fue fijado inicialmente en $61 \mathrm{R}_{\odot}$ y luego levemente variado para obtener el ajuste deseado.

Cabe mencionar que $\mathrm{v}_{\text {mic }}$ fue fijada inicialmente en $10 \mathrm{~km} / \mathrm{s}$ y que a la hora de variar, por ejemplo, el radio estelar y la gravedad superficial tuvimos en cuenta que ambos parámetros deberían seguir la relación $g R_{\star}^{2} \approx$ constante.

Para determinar las parámetros del viento, $\dot{M}$ y $v_{\infty}$, utilizamos el perfil de la línea de $\mathrm{H}_{\alpha}$. Para ajustar la velocidad terminal, consideramos preferentemente los valores que reproducen la componente de absorción desplazada hacia el azul del perfil P Cygni. En cambio si el perfil está en emisión pura derivamos la velocidad terminal principalmente del ancho de la línea $\mathrm{H}_{\alpha}$. La tasa de pérdida de masa la derivamos a partir del ajuste de la componente en emisión.

\subsubsection{Parámetros estelares}

En promedio hemos modelado la fotosfera de la estrella con una temperatura media de $18800 \mathrm{~K}$, con variaciones en $\mathrm{T}_{\text {ef }}$ que van desde $18570 \mathrm{~K}$ a $19100 \mathrm{~K}$ (ver tablas 4.1. $\mathrm{y}$ 4.2 . Encontramos un valor medio de $\log \mathrm{g}=2.43$ dex con una dispersión de \pm 0.14 dex. Con respecto al radio, usando un valor inicial de $61 \mathrm{R}_{\odot}$ que luego fuimos variando para lograr un mejor ajuste, obtuvimos un valor de $57 \mathrm{R}_{\odot}$, cuando empleamos las observaciones de Arizona. Sin embargo, como contábamos con un mayor número de observaciones de Ondřejov, no pudimos mantener fijo el radio dada la tremenda variación de los perfiles, lo que nos condujo a proponer valores entre $52 \mathrm{R}_{\odot}$ y $63 \mathrm{R}_{\odot}$. Con el valor adoptado para el radio y la gravedad efectiva, determinamos una masa de $34 \pm 4 \mathrm{M}_{\odot}$, mientras que la luminosidad promedio encontrada fue de $\mathrm{L} / \mathrm{L}_{\odot}=5.57 \pm 0.03$.

\subsubsection{Variación del viento}

Para analizar la variabilidad del viento de $55 \mathrm{Cyg}$, modelamos el perfil de línea de $\mathrm{H}_{\alpha}$ observado a lo largo de un período de casi 5 años. Para ajustar las observaciones hemos supuesto la ausencia de clumping y una estructura del viento representada por un campo de velocidades con exponente $\beta=2$. En promedio hemos determinado $\dot{\mathrm{M}} \sim 2.5 \times 10^{-7}$ 
$\mathrm{M}_{\odot} /$ año, con fluctuaciones entre un valor mínimo de $1.5 \times 10^{-7} \mathrm{M}_{\odot} /$ año y uno máximo de $4.6 \times 10^{-7} \mathrm{M}_{\odot} /$ año. Estos resultados, en promedio, están de acuerdo con los valores obtenidos por Crowther et al. 2006a tabla 3): $\beta=2$ y $\dot{M}=2.3 \times 10^{-7} \mathrm{M}_{\odot} /$ año. En lo que respecta a la velocidad terminal determinada, ésta toma valores entre $230 \mathrm{~km} / \mathrm{s}$ y 350 $\mathrm{km} / \mathrm{s}$, pero hay dos excepciones con velocidades terminales muy altas: $600 \mathrm{~km} / \mathrm{s}$ y 700 $\mathrm{km} / \mathrm{s}$, correspondientes a dos ocasiones de altos valores de pérdida de masa: $4.6 \times 10^{-7}$ $\mathrm{M}_{\odot} /$ año y 4 x $10^{-7} \mathrm{M}_{\odot} /$ año. Las correspondientes observaciones fueron el 25/9/2011 y el 26/2/2012, respectivamente, cuando probablemente ocurrió un proceso eruptivo.

\subsubsection{Velocidades de turbublencia y rotación}

De nuestros ajustes, encontramos que las líneas de He pueden ser modeladas con un valor de $\mathrm{v}_{\text {mic }}=10 \pm 2 \mathrm{~km} / \mathrm{s}$, excepto la línea HeI $\lambda 6678 \AA$ que requiere un valor más alto $(17 \pm 2 \mathrm{~km} / \mathrm{s})$. Tampoco fue posible obtener ajustes satisfactorios para la línea $\mathrm{H}_{\alpha}$ con valores tan bajos de microturbulencia. En este caso hemos tenido que recurrir a un valor de $50 \mathrm{~km} / \mathrm{s}$ que puede ser explicado por el hecho de que $\mathrm{H}_{\alpha}$ se forma en la parte más baja del viento donde pueden existir velocidades turbulentas supersónicas. Valores tan altos de $\mathrm{v}_{\text {mic }}$ $(50 \mathrm{~km} / \mathrm{s})$ ya han sido reportados para muchas estrellas BSGs (por ejemplo en, Crowther et al. 2006b Searle et al. 2008b. Para las líneas $\mathrm{H}_{\alpha}$ y He I $\lambda 6678 \AA$, interpretamos que los valores altos de $\mathrm{v}_{\text {mic }}$ que derivamos surgen debido a que éstas líneas están afectadas por la dispersión de velocidad del fluido.

Después de fijar el valor de $\mathrm{v}_{\text {mic }}$, incorporaramos a los perfiles sintéticos los efectos de ensanchamiento combinados de la velocidad de macroturbulencia y rotación. Teniendo en cuenta que la velocidad de rotación estelar proyectada, vsen i, es un parámetro global, es decir, es el mismo para todas las líneas, y que observamos variaciones en todas las líneas, atribuimos que esta variabilidad se debe a cambios en la velocidad de macroturbulencia. Por lo tanto, el mejor ajuste para todas las líneas fue logrado con una $v$ sen $i=55 \pm 5 \mathrm{~km} / \mathrm{s}$, mientras que para la $\mathrm{v}_{\mathrm{mac}}$ fue necesario, para cada fecha, adoptar diferentes valores para reproducir de manera óptima el ancho de los perfiles. Por ejemplo, para el modelado de las observaciones adquiridas en Arizona, encontramos que el valor de $\mathrm{v}_{\text {mac }}$ es relativamente chico en las líneas de HeI $\lambda 4471 \AA(6-7 \mathrm{~km} / \mathrm{s})$, He I $\lambda 4713 \AA(11-12 \mathrm{~km} / \mathrm{s}), y \mathrm{H}_{\delta}(14-15$ $\mathrm{km} / \mathrm{s}$ ), y prácticamente constante a lo largo de todo el período de observación. En $\mathrm{H}_{\gamma}$ los cambios desplegados por este campo de velocidades son mayores (14-30 km/s). Sin embargo, el análisis de este parámetro en las líneas $\mathrm{H}_{\beta}$ y He I $\lambda 6678$ muestra que los valores cambian para las diferentes épocas entre $25-90 \mathrm{~km} / \mathrm{s}$ y $20-50 \mathrm{~km} / \mathrm{s}$, respectivamente, mientras que para $\mathrm{H}_{\alpha}$ varían entre $20-60 \mathrm{~km} / \mathrm{s}$ (ver tabla 4.2). Por otro lado, las líneas de Si II y Si III también muestran una gran dispersión en $\mathrm{v}_{\mathrm{mac}}$, que va de unos $10 \mathrm{~km} / \mathrm{s}$ a $30 \mathrm{~km} / \mathrm{s}$ y de 30 $\mathrm{km} / \mathrm{s}$ a $50 \mathrm{~km} / \mathrm{s}$, respectivamente, con incertezas de $\pm 5 \mathrm{~km} / \mathrm{s}$.

Analizando los datos de Ondřejov, éstos sólo proveen valores de $\mathrm{v}_{\text {mac }}$ para $\mathrm{H}_{\alpha} \mathrm{y}$ He I $\lambda 6678$. A lo largo de todo el período de observación obtenemos valores entre $30 \mathrm{~km} \mathrm{~s}^{-1}$ y $60 \mathrm{~km} \mathrm{~s}^{-1}$ para el HeI, mientras que para el H, ocasionalmente encontramos valores de más de 100 $\mathrm{km} \mathrm{s}^{-1}$ (ver tabla 4.1). En la mayoría de los espectros encontramos que para ambas líneas la contribución de la velocidad de macroturbulencia al ensanchamiento de las líneas es 
comparable o mayor que la velocidad de rotación proyectada. Estos grandes valores son comúnmente encontrados en las BSGs (por ejemplo, en Ryans et al. 2002 Simón-Díaz \& Herrero 2007b Markova et al. 2008a 2014 Simón-Díaz \& Herrero 2014b), y los valores de $\mathrm{v} \sin i$ y $\mathrm{v}_{\mathrm{mac}}$ encontrados para 55 Cyg están de acuerdo con los de aquellas BSGs de masa y temperatura comparables a 55 Cyg (ver Markova et al. 2014.

\subsubsection{Ajuste de perfiles}

En esta sección mostramos todos los ajustes obtenidos mediante el procedimiento descripto previamente. Las figs. 4.2, 4.3, 4.4, 4.5 y 4.6 despliegan los ajustes realizados a los perfiles observados de $\mathrm{H}_{\alpha}$ adquiridos en el Observatorio de Ondřejov para los años 2009, 2010, 2011, 2012 y 2013, respectivamente. En estas figuras la observación está en negro, mientras que el mejor modelo ajustado está en rojo. En algunos casos no hemos podido encontrar un modelo apropiado que ajuste satisfactoriamente el perfil observado.

Por otro lado, a los ajustes de las observaciones adquiridas en Arizona, que cubren un rango mayor en longitud de onda, las separamos en dos subgrupos. En el primer subgrupo están los ajustes de todas las líneas de hidrógeno (figs. 4.7 4.8 4.9), mientras que en el otro subgrupo podemos entontrar las líneas de He (figs. 4.10 4.11 y 4.12 y de Si (figs. 4.13 , 4.14 y 4.15.

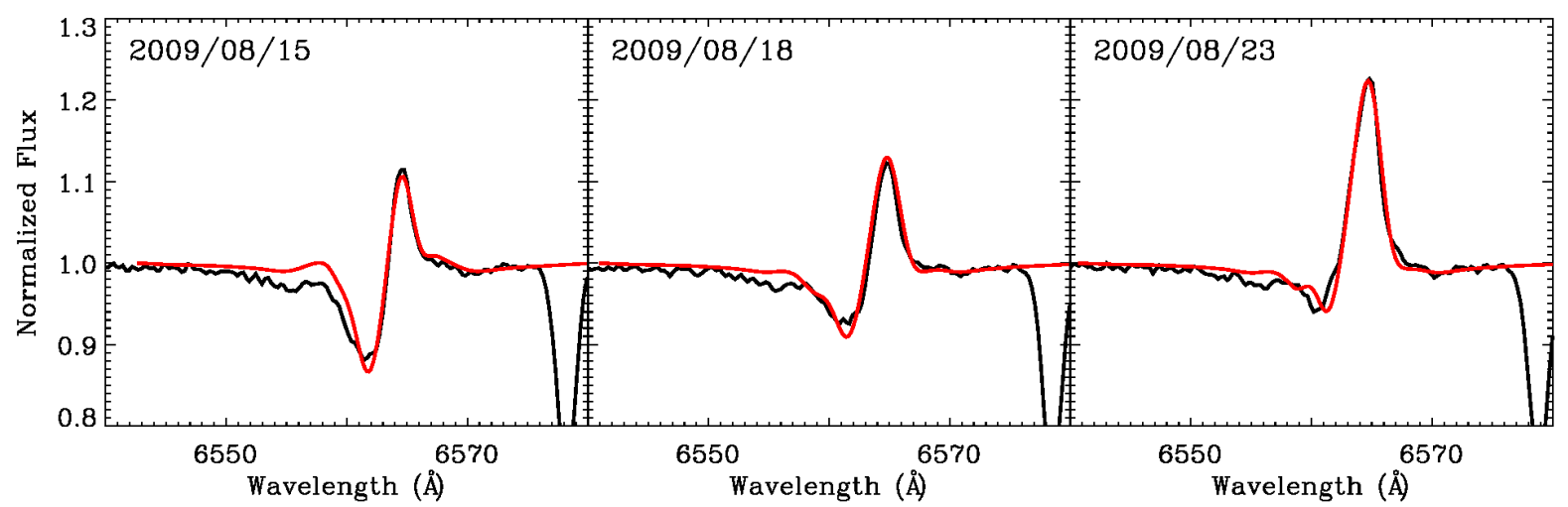

Figura 4.2. Ajustes del perfil de línea de $\mathrm{H}_{\alpha}$ teórico al observado en 2009. El modelo y la observación están representados en rojo y negro, respectivamente. 


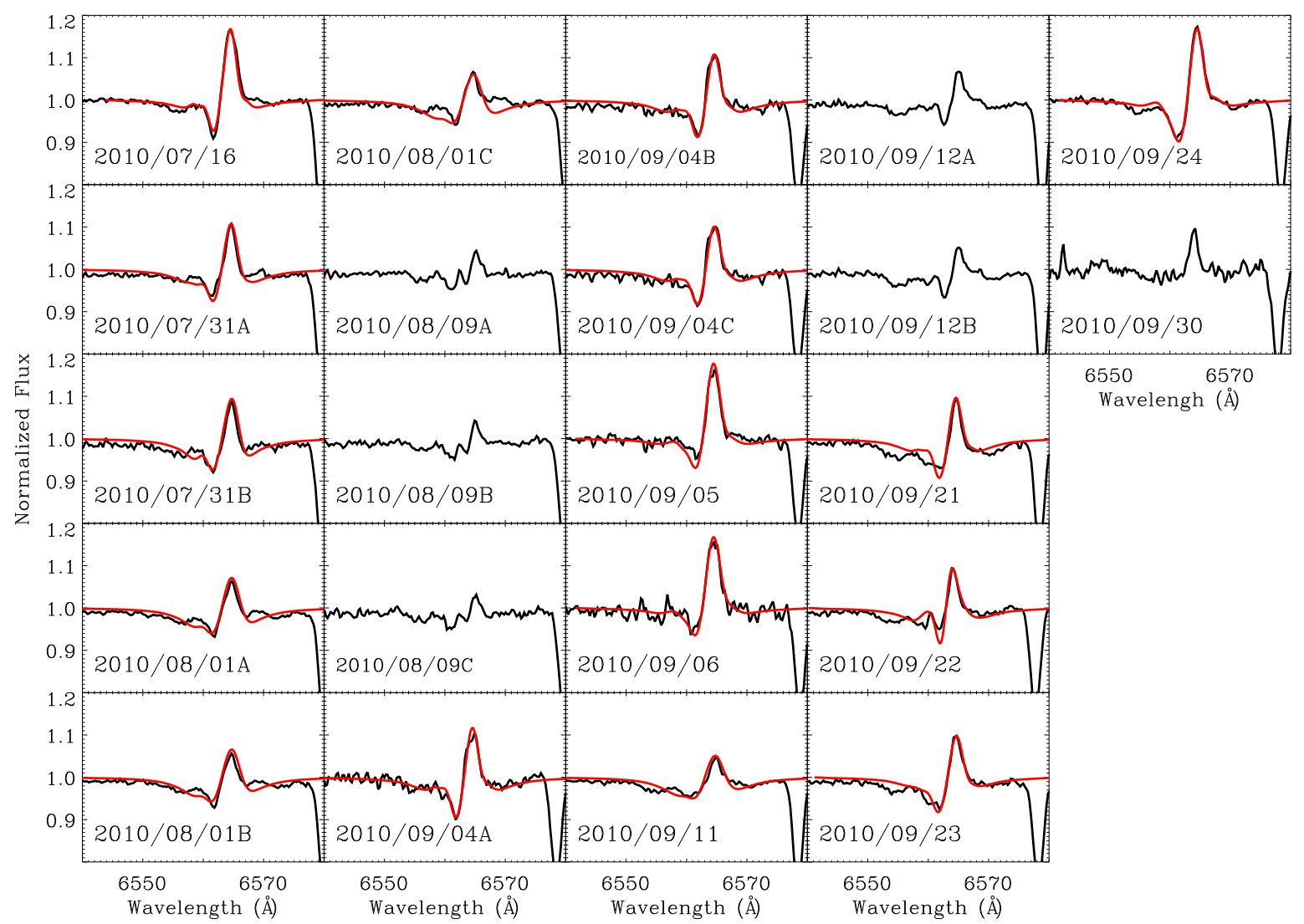

Figura 4.3. Ajustes del perfil de línea de $\mathrm{H}_{\alpha}$ teórico al observado en 2010. El modelo y la observación están representados en rojo y negro, respectivamente. 


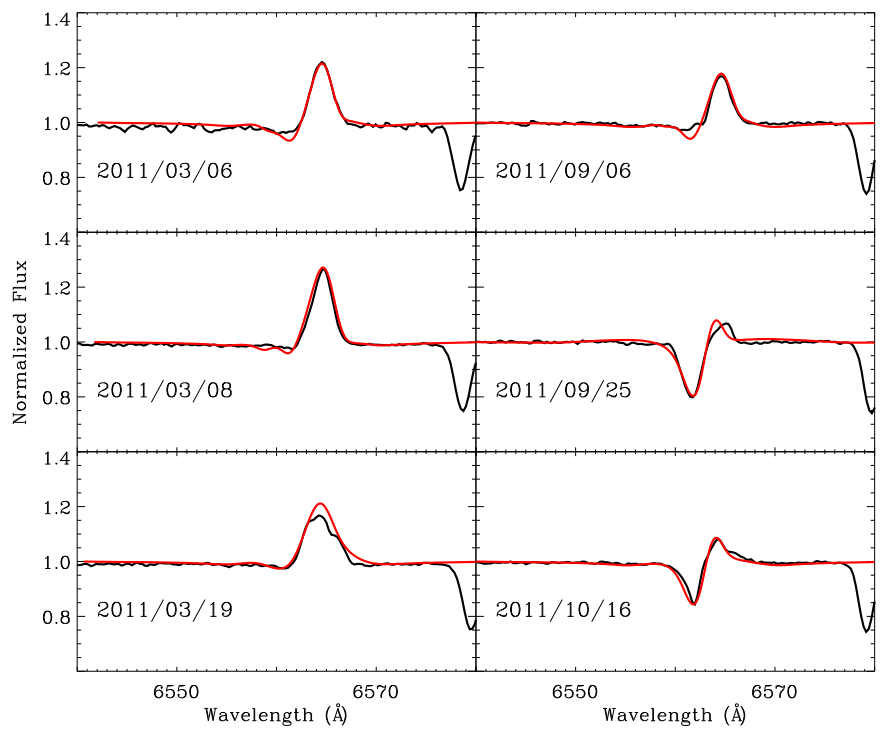

Figura 4.4. Ajustes del perfil de línea de $\mathrm{H}_{\alpha}$ teórico al observado en 2011. El modelo y la observación están representados en rojo y negro, respectivamente.

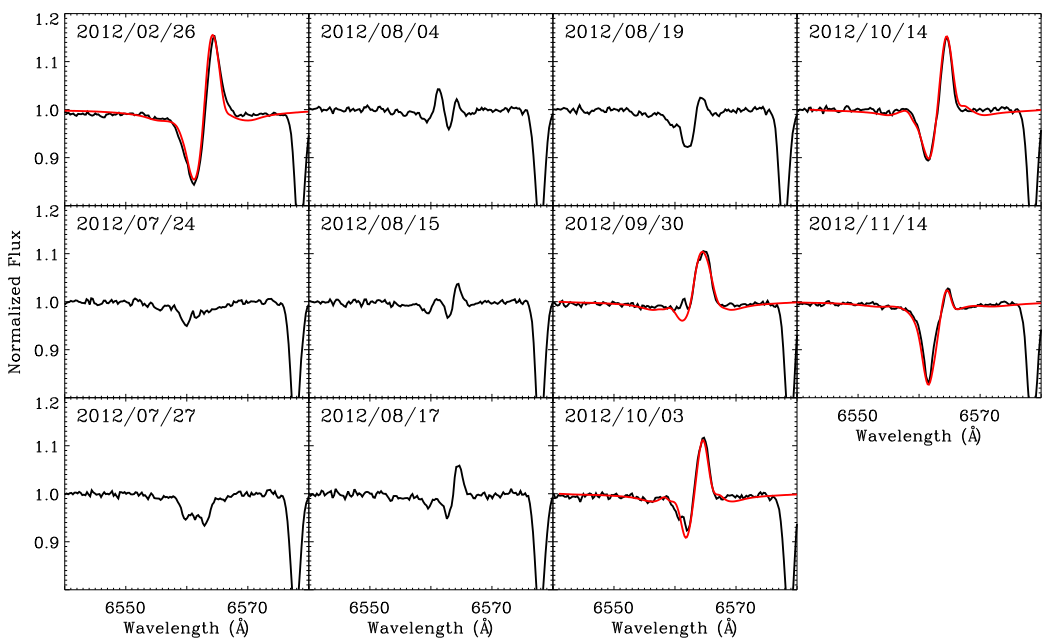

Figura 4.5. Ajustes del perfil de línea de $\mathrm{H}_{\alpha}$ teórico al observado en 2012. El modelo y la observación están representados en rojo y negro, respectivamente. 


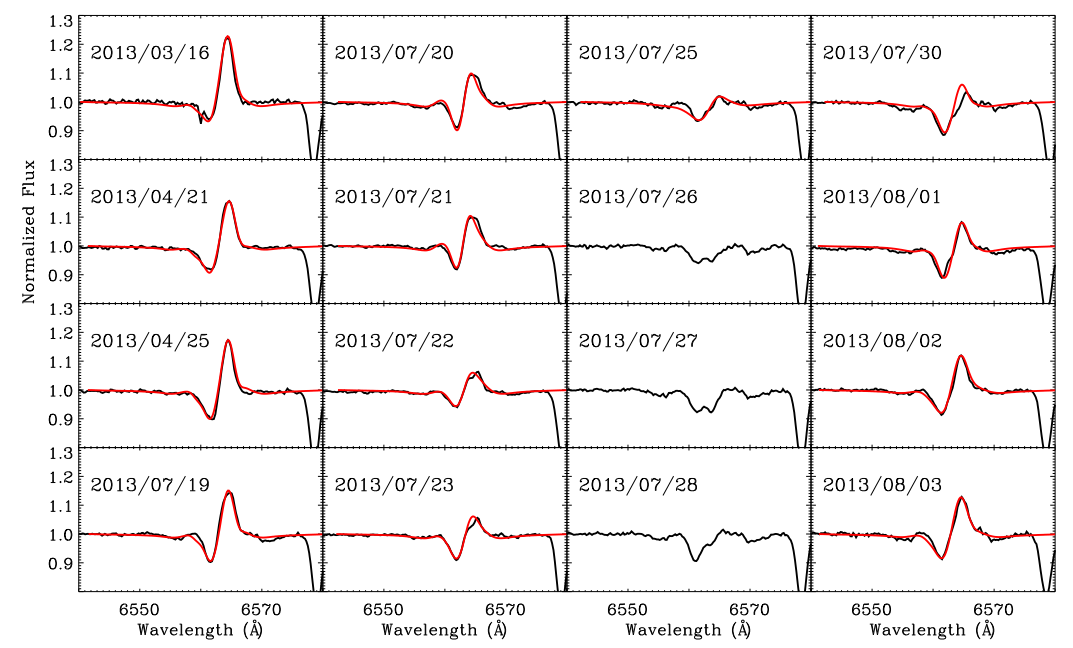

Figura 4.6. Ajustes del perfil de línea de $\mathrm{H}_{\alpha}$ teórico al observado en 2013. El modelo y la observación están representados en rojo y negro, respectivamente.

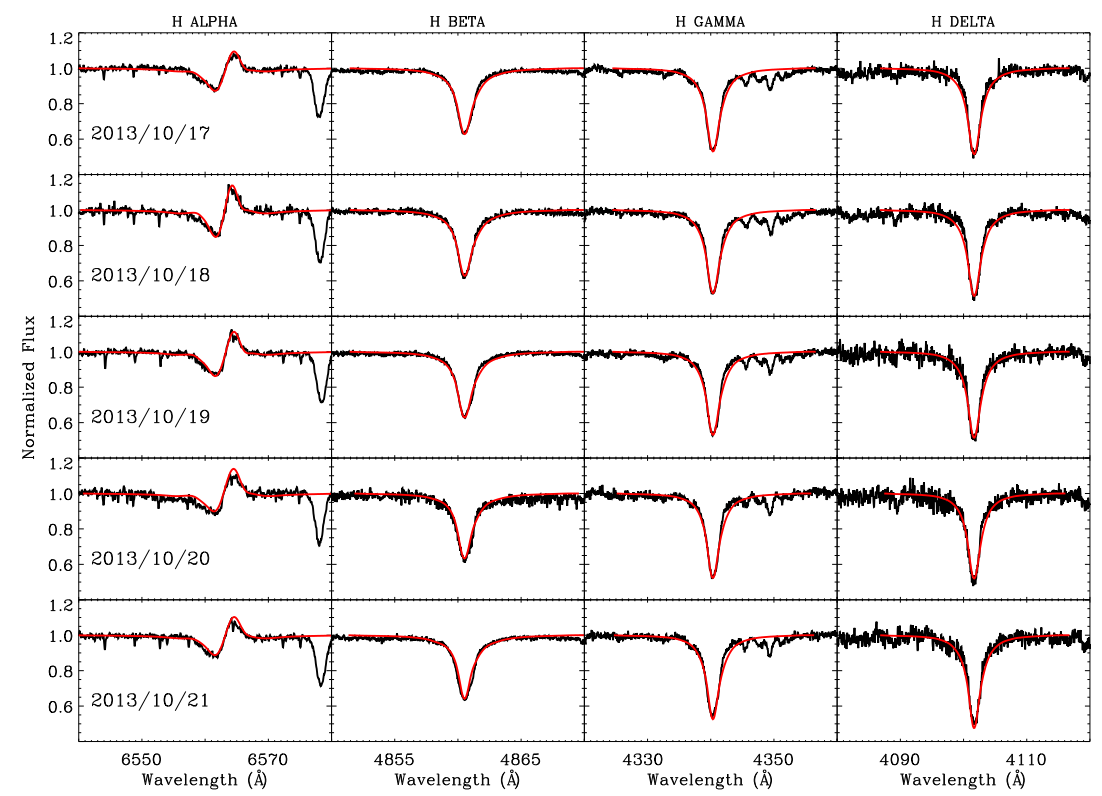

Figura 4.7. Ajustes del espectro de $\mathrm{H}\left(\mathrm{H}_{\alpha}, \mathrm{H}_{\beta}, \mathrm{H}_{\gamma}\right.$ y $\left.\mathrm{H}_{\delta}\right)$ observado en Arizona. El modelo y la observación están representados en rojo y negro, respectivamente. 


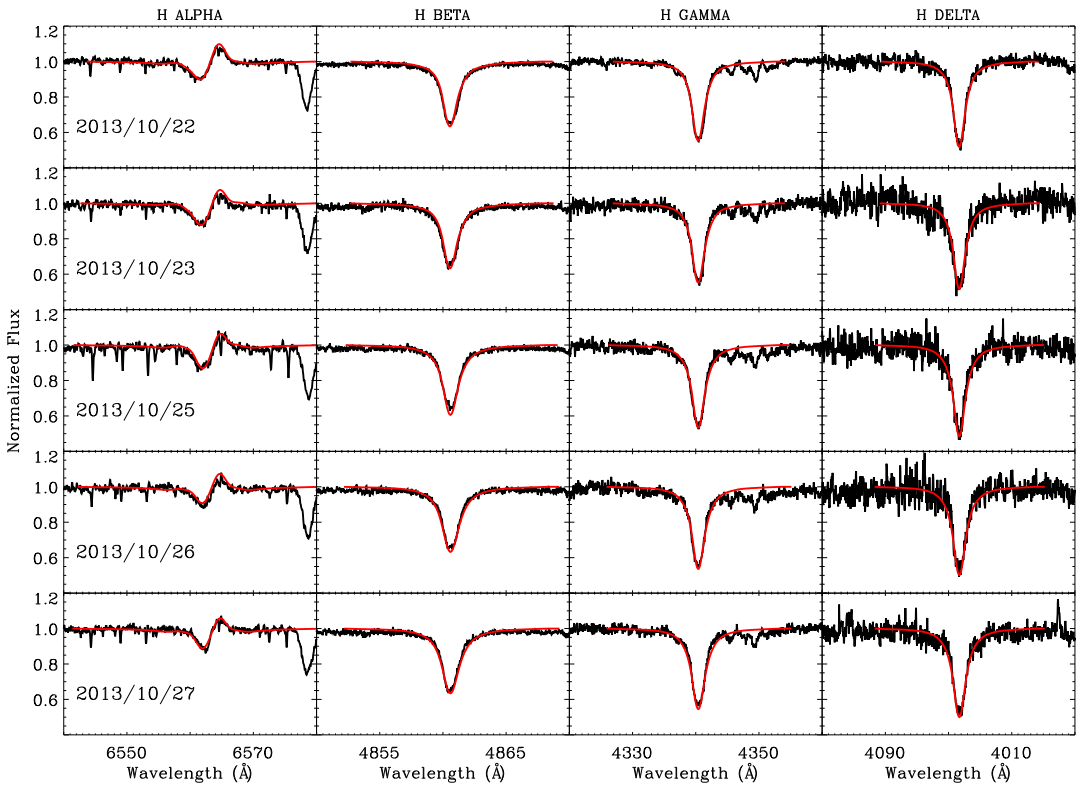

Figura 4.8. Ajustes del espectro de $\mathrm{H}\left(\mathrm{H}_{\alpha}, \mathrm{H}_{\beta}, \mathrm{H}_{\gamma}\right.$ y $\left.\mathrm{H}_{\delta}\right)$ observado en Arizona. El modelo y la observación están representados en rojo y negro, respectivamente.

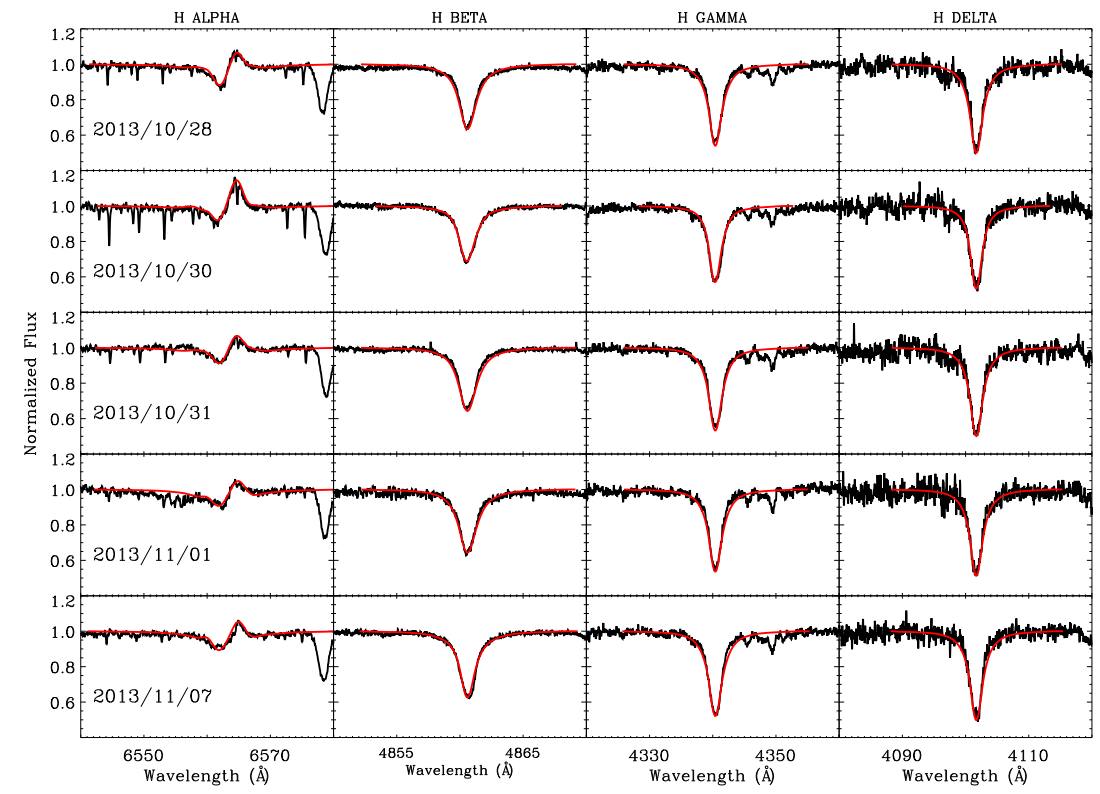

Figura 4.9. Ajustes del espectro de $\mathrm{H}\left(\mathrm{H}_{\alpha}, \mathrm{H}_{\beta}, \mathrm{H}_{\gamma}\right.$ y $\left.\mathrm{H}_{\delta}\right)$ observado en Arizona. El modelo y la observación están representados en rojo y negro, respectivamente. 


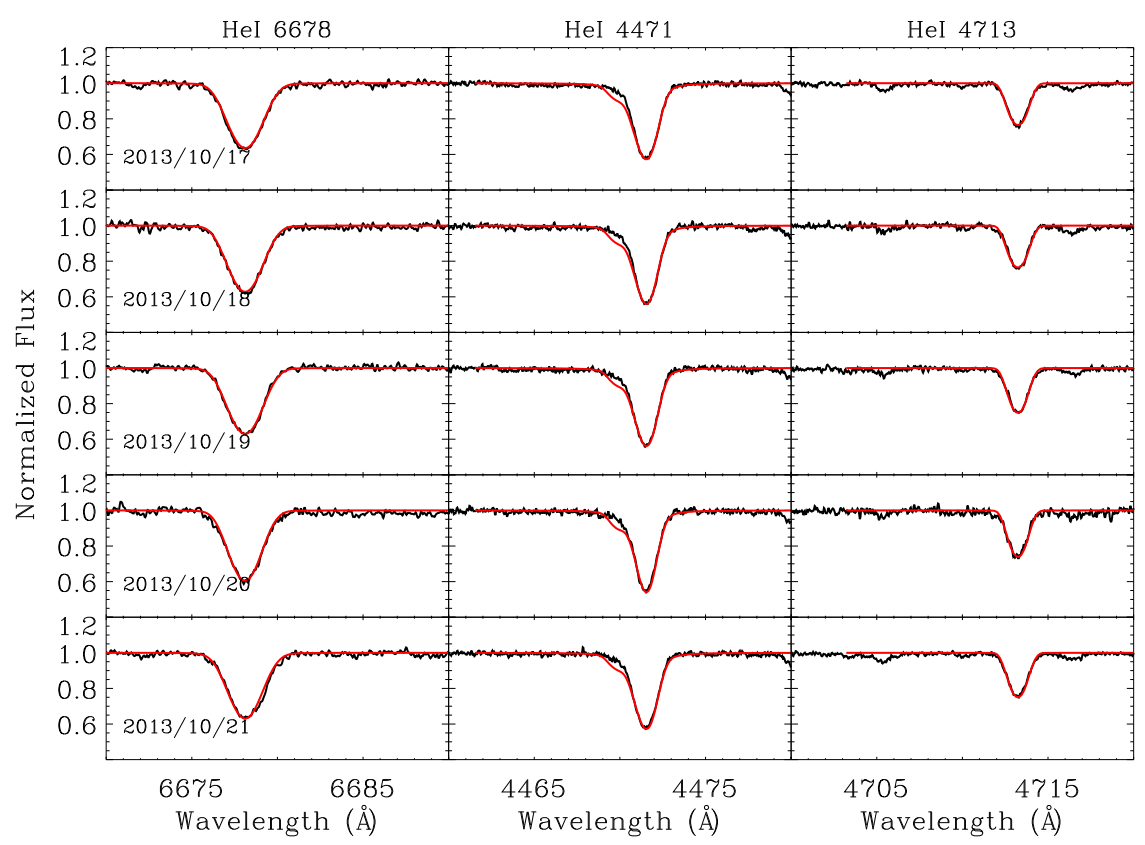

Figura 4.10. Ajustes de los perfiles de líneas de HeI $\lambda 5676 \AA$, He I $\lambda 4471 \AA$ y HeI $\lambda 4713 \AA$. El modelo y la observación están representados en rojo y negro, respectivamente.

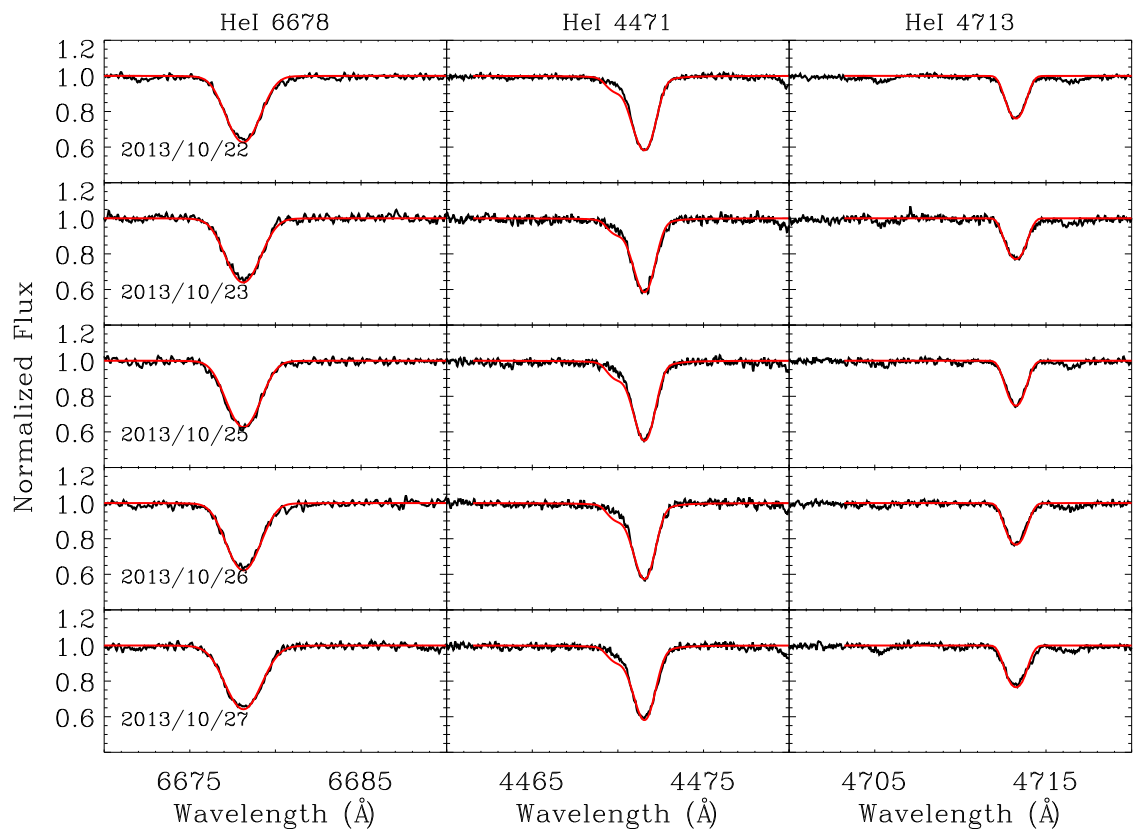

Figura 4.11. Ajustes de los perfiles de líneas de He I $\lambda 5676 \AA$, He I $\lambda 4471 \AA$ y He $\mathrm{I} \lambda 4713 \AA$. El modelo y la observación están representados en rojo y negro, respectivamente. 


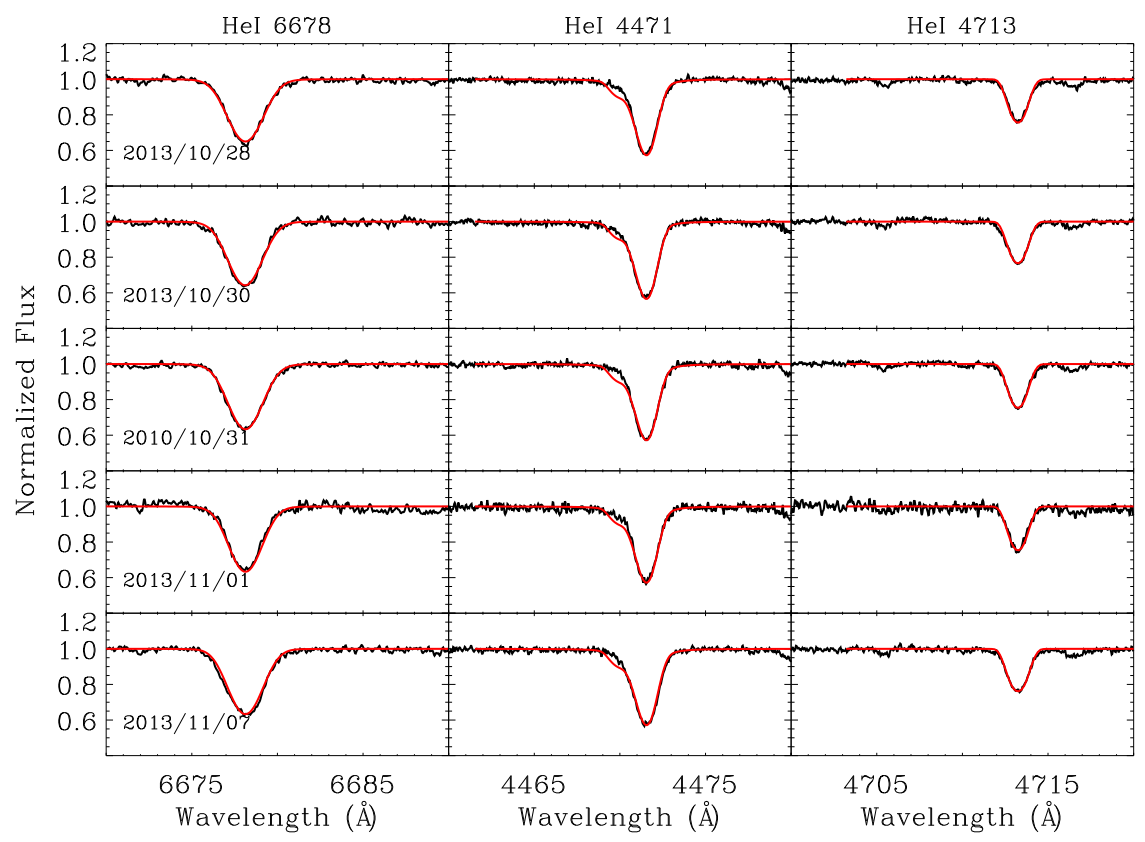

Figura 4.12. Ajustes de los perfiles de líneas de He, $\lambda 5676 \AA$, He I $\lambda 4471 \AA$ y $\operatorname{HeI} \lambda 4713 \AA$. El modelo y la observación están representados en rojo y negro, respectivamente.

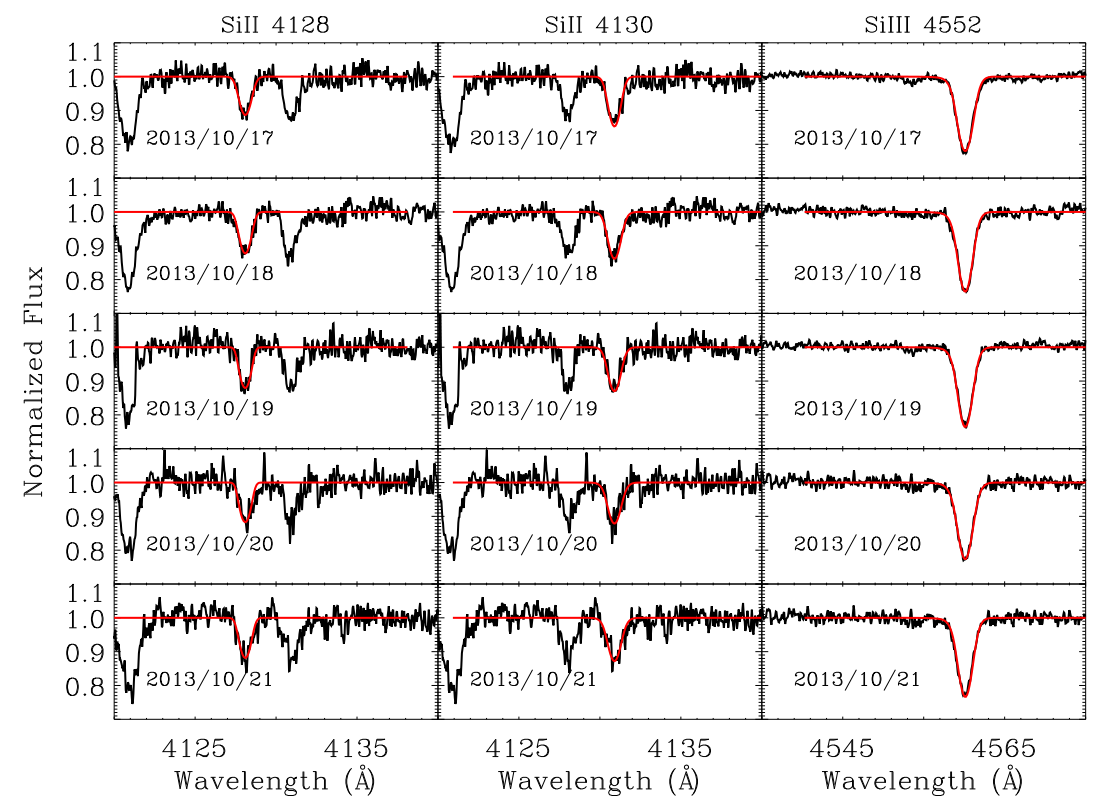

Figura 4.13. Ajustes de los perfiles de líneas de Si III $\lambda \lambda 4128,4130 \AA$, Si III $\lambda 4552 \AA$. El modelo y la observación están representados en rojo y negro, respectivamente. 


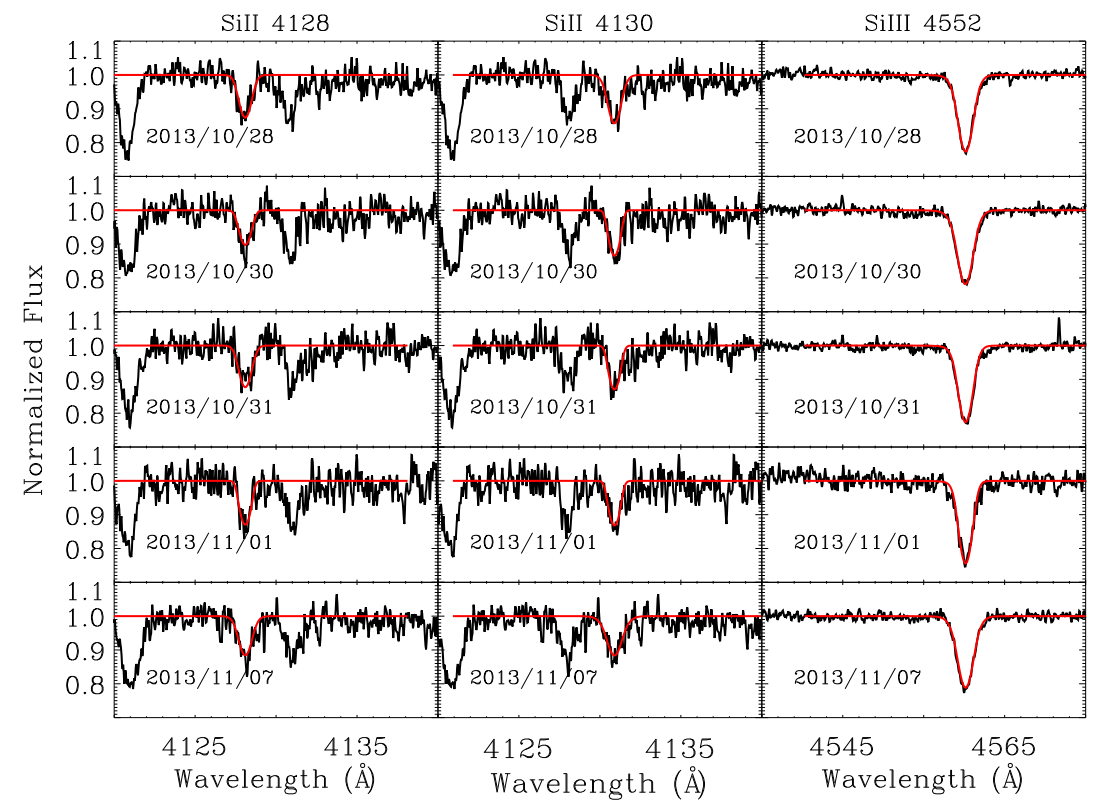

Figura 4.14. Ajustes de los perfiles de líneas de Si III $\lambda \lambda 4128,4130 \AA$, Si III $\lambda 4552 \AA$. El modelo y la observación están representados en rojo y negro, respectivamente.

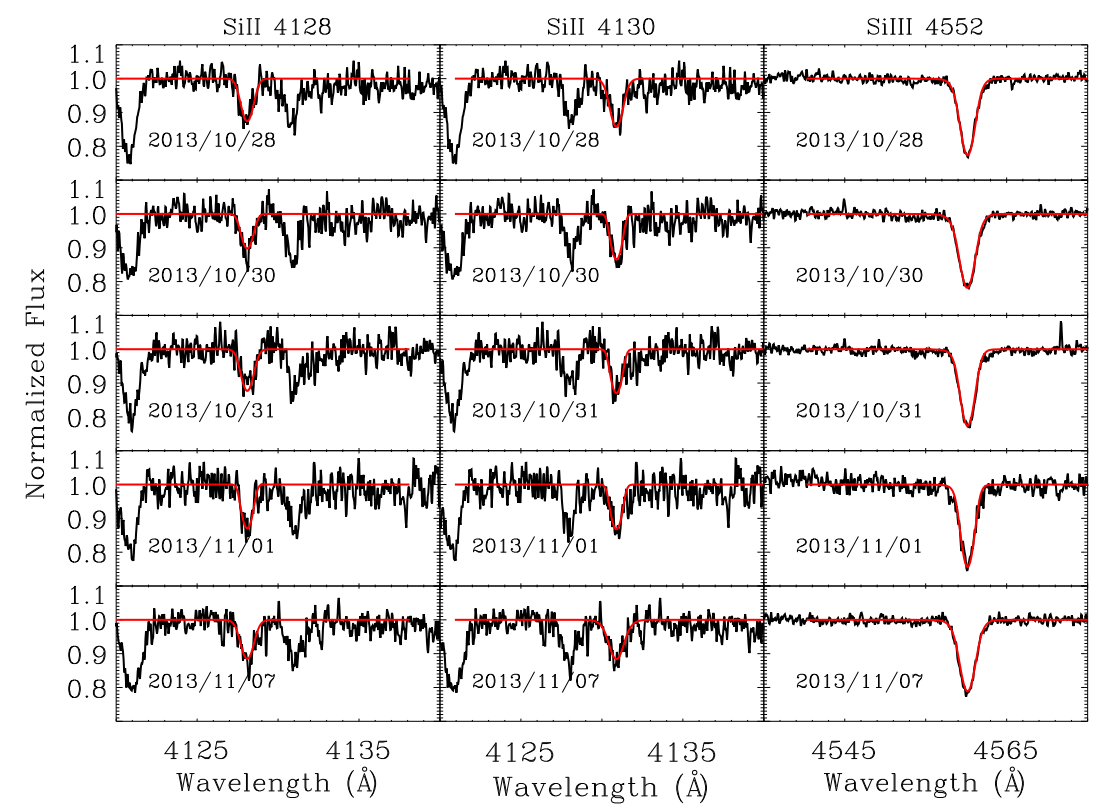

Figura 4.15. Ajustes de los perfiles de líneas de Si III $\lambda \lambda 4128,4130 \AA$, Si III $\lambda 4552 \AA$. El modelo y la observación están representados en rojo y negro, respectivamente. 


\subsubsection{Errores}

Usando el código FASTWIND exploramos la sensibilidad de los parámetros que caracterizan a la fotosfera y al viento. Sin embargo, no debemos olvidar que existen dos fuentes de error. Una resulta del modelado, en donde las incertezas involucradas surgen de la desviación estándar estadística y son obtenidos usando el mejor modelo ajustado. La segunda fuente se refiere a la imprecisión de los parámetros de entrada, principalmente del radio, que resulta de incertezas en la distancia. Pero una vez que la distancia es fijada, cualquier desviación de los parámetros que sea mayor que los errores estadísticos, indica posibles variaciones de los parámetros físicos.

Para determinar la incerteza estadística de los parámetros, usamos el mejor modelo ajustado y variamos los diferentes valores de cada parámetro de a uno por vez, manteniendo fijo los restantes. Tenemos la ventaja en este caso de tener muchos espectros de alta resolución y alta $\mathrm{S} / \mathrm{N}$ tomados durante días consecutivos. Esto nos permitió distinguir entre errores sobre los valores medios de los parámetros estudiados y cambios reales en las condiciones físicas.

Cabe mencionar que para evaluar el error en $\dot{M}$ hicimos una propagación de errores usando el parámetro de profundidad óptica invariante $\mathrm{Q}=\dot{\mathrm{M}} /\left(\mathrm{v}_{\infty} \mathrm{R}_{\star}\right)^{1,5}$, que resulta de los cálculos del modelo (ver cap. 2, sec. 2.9). Considerando una dispersión en la magnitud bolométrica de $\Delta \mathrm{M}_{\text {bol }} \simeq \pm 0.3 \mathrm{mag}, \Delta \log \mathrm{L} / \mathrm{L}_{\odot} \simeq \pm 0.12$, y un error en $\Delta \mathrm{T}_{\mathrm{ef}} \simeq$ $\pm 500 \mathrm{~K}$, obtenemos $\Delta \log \mathrm{R}_{\star} / \mathrm{R}_{\odot} \simeq \pm 0.04$. Con nuestra incerteza del $10 \%$ en $\mathrm{v}_{\infty}$ y un error estadístico de $\Delta \log \mathrm{Q} \simeq \pm 0.07$, la incerteza en la tasa de pérdida de masa es $\Delta \dot{\mathrm{M}} / \dot{\mathrm{M}} \simeq 0.3$.

De esta manera, los errores que determinamos para cada uno de los parámetros son los siguientes:

- $\Delta \mathrm{T}_{\mathrm{ef}}= \pm 300-500 \mathrm{~K}$.

- $\Delta \log g= \pm 0.05$ dex.

- $\Delta \mathrm{R}_{\star}= \pm 8 \mathrm{R}_{\odot}$ (el radio se obtuvo a partir del método BCD).

- $\Delta \mathrm{L} / \mathrm{L}_{\odot}= \pm 0.1$.

- $\Delta \mathrm{v}_{\text {mic }}= \pm 2 \mathrm{~km} / \mathrm{s}$ (para la líneas de He y $\mathrm{Si}$ ) y $\pm 5 \mathrm{~km} / \mathrm{s}$ (para H excepto $\mathrm{H}_{\alpha}$ ).

- $\Delta \mathrm{v}_{\text {mac }} \mathrm{y} \Delta$ (vsen i) (velocidad de rotación proyectada) $= \pm 5-10 \mathrm{~km} / \mathrm{s}$ (para He-Si e $\mathrm{H}$, respectivamente).

- $\Delta \dot{M}= \pm 0.1 \times 10^{-7} M_{\odot} /$ año.

- $\Delta \mathrm{v}_{\infty} / \mathrm{v}_{\infty}= \pm 10 \%$.

Todos los parámetros estelares y del viento obtenidos en este capítulo, junto con la estimación de sus errores pueden apreciarse en las tablas 4.1 y 4.2. Los valores de la velocidad de macroturbulencia de esta tabla se refieren al ajuste del perfil $\mathrm{H}_{\alpha}$. 
Tabla 4.1. Parámetros estelares y del viento de 55 Cyg.

\begin{tabular}{|c|c|c|c|c|c|c|c|c|c|}
\hline & & & & & & & & & \\
\hline $\begin{array}{c}\text { Época } \\
\mathrm{dd} / \mathrm{mm} / \mathrm{aa}\end{array}$ & DJ & $\begin{array}{l}T_{\text {ef }} \\
{[\mathrm{K}]}\end{array}$ & $\begin{array}{l}\log g \\
{[\operatorname{cgs}]}\end{array}$ & $\beta$ & $\begin{array}{c}\dot{\mathrm{M}} \\
{\left[10^{-6} M_{\odot} / \mathrm{año}\right]}\end{array}$ & $\begin{array}{c}\mathrm{v}_{\infty} \\
{[\mathrm{km} / \mathrm{s}]}\end{array}$ & $\begin{array}{c}\mathrm{v}_{\operatorname{mac}}\left(\mathrm{H}_{\alpha}\right) \\
{[\mathrm{km} / \mathrm{s}]}\end{array}$ & $\begin{array}{c}\mathrm{R}_{\star} \\
{\left[\mathrm{R}_{\odot}\right]}\end{array}$ & $\log \mathrm{L} / \mathrm{L}_{\odot}$ \\
\hline $15 / 08 / 2009$ & 455059.44 & $000 \pm 1000$ & $.40 \pm 0,05$ & 2.0 & $0.290 \pm 0,01$ & $310 \pm 30$ & $35 \pm 5$ & $58 \pm 2$ & $5.60 \pm 0.03$ \\
\hline $18 / 08 / 2009$ & 2455062.43 & $19000 \pm 1000$ & $2.43 \pm 0,05$ & 2.0 & $95 \pm 0,01$ & & \pm 5 & $54 \pm 2$ & $5.54 \pm 0.03$ \\
\hline $23 / 08 / 2009$ & 2455067.49 & $19000 \pm 1000$ & $2.43 \pm 0,05$ & 2.0 & $0.340 \pm 0,01$ & $320 \pm 30$ & $30 \pm 5$ & $55 \pm 2$ & $5.55 \pm 0.03$ \\
\hline $16 / 07 / 2010$ & 2455394.41 & $18700 \pm 1000$ & $2.35 \pm 0,05$ & 2.0 & $40 \pm 0$ & $250 \pm 25$ & $14 \pm 5$ & $63 \pm 2$ & $5.64 \pm 0.03$ \\
\hline $31 \mathrm{~A} / 07 / 2010$ & 2455409.37 & $18700 \pm 1000$ & $2.50 \pm 0,05$ & 2.0 & $5 \pm 0,01$ & 250 & \pm 5 & $57 \pm 2$ & $5.56 \pm 0.03$ \\
\hline $31 \mathrm{~B} / 07 / 2010$ & 2455409.53 & $18700 \pm 1000$ & $2.50 \pm 0,05$ & 2.0 & $95 \pm 0,01$ & $250 \pm 25$ & $40 \pm 5$ & $57 \pm 2$ & $5.56 \pm 0.03$ \\
\hline $01 \mathrm{~A} / 08 / 2010$ & 2455410.35 & $18700 \pm 1000$ & $2.50 \pm 0$ & 2.0 & $95 \pm 0,02$ & $250 \pm 50$ & $80 \pm 10$ & $57 \pm 3$ & $5.56 \pm 0.06$ \\
\hline 01B/08/2010 & 2455410.45 & $18700 \pm 1000$ & $2.50 \pm 0$ & 2.0 & \pm 1 & 250 & $90 \pm 10$ & $57 \pm 3$ & $5.56 \pm 0.06$ \\
\hline $01 \mathrm{C} / 08 /$ & 2455410.45 & $0 \pm 1000$ & $2.50 \pm 0$ & 2.0 & \pm 0 & & \pm 10 & $57 \pm 3$ & $5.56 \pm 0.06$ \\
\hline 04A/09/2010 & 2455444.38 & $18650 \pm 1000$ & $2.50 \pm 0,05$ & 2.0 & 1 & & \pm 5 & $56 \pm 2$ & $5.54 \pm 0.03$ \\
\hline 04B/09/2010 & 2455444.41 & $18650 \pm 1000$ & $2.50 \pm 0$ & 2.0 & 01 & & $30 \pm 5$ & $56 \pm 2$ & $=0.03$ \\
\hline $04 \mathrm{C} / 09 / 2010$ & 2455444.45 & $18650 \pm 1000$ & $2.50 \pm 0$ & 2.0 & \pm 0 & 27 & $40 \pm 5$ & $56 \pm 2$ & $5.54 \pm 0.03$ \\
\hline $05 / 09 / 2$ & 245544 & 190 & $2.41 \pm 0$ & 2.2 & \pm( & & $50 \pm 5$ & $55 \pm 2$ & $5.55 \pm 0.03$ \\
\hline 06/09/2010 & 2455446.52 & $19000 \pm 1000$ & $2.41 \pm 0$ & 2.2 & 1 & & $60 \pm 5$ & $55 \pm 2$ & \pm 0.03 \\
\hline $11 / 09 / 2010$ & 2455451.27 & $18600 \pm 1000$ & $2.50 \pm 0$ & 2.0 & $5 \pm 0$ & & $120 \pm 10$ & $57 \pm 2$ & $5.55 \pm 0.03$ \\
\hline $21 / 09 / 2010$ & 2455461.34 & $18600 \pm 10$ & $2.40 \pm 0$ & 2.0 & +0 & & \pm 5 & $58 \pm 3$ & $5.56 \pm 0.06$ \\
\hline $22 / 09 / 2$ & 245546 & $18600 \pm 1000$ & $2.48 \pm 0$ & 2.0 & 2 & 23 & \pm 5 & $58 \pm 3$ & $5.64 \pm 0.06$ \\
\hline $23 / 09 / 2010$ & 2455463.38 & $18700 \pm 1000$ & $2.42 \pm 0,05$ & 2.0 & $5 \pm 0$ & & $50 \pm 5$ & $63 \pm 2$ & $5.56 \pm 0.03$ \\
\hline $24 / 09 / 2010$ & 2455464.38 & $19000 \pm 1000$ & $2.45 \pm 0,05$ & 2.0 & $0.305 \pm 0,01$ & $330 \pm 35$ & $35 \pm 5$ & $54 \pm 2$ & $5.54 \pm 0.03$ \\
\hline $06 / 03 / 2011$ & 2455627.64 & $19000 \pm 1000$ & $2.43 \pm 0$ & 2.0 & $5 \pm 0$ & $320 \pm 30$ & $30 \pm 5$ & $55 \pm 2$ & $5.55 \pm 0.03$ \\
\hline $08 / 03 / 2011$ & 2455629.60 & $19000 \pm 1000$ & $2.43 \pm 0,05$ & 2.0 & $0 \pm 0,01$ & 32 & $20 \pm 5$ & $55 \pm 2$ & $5.55 \pm 0.03$ \\
\hline $19 / 03 / 2011$ & 2455640.63 & $19000 \pm 1000$ & $2.43 \pm 0,05$ & 2.0 & $70 \pm 0,01$ & $320 \pm 30$ & $100 \pm 10$ & $55 \pm 2$ & $5.57 \pm 0.03$ \\
\hline $06 / 09 / 2011$ & 2455811.40 & $18700 \pm 1000$ & $2.46 \pm 0$ & 2.0 & $35 \pm 0,01$ & & $30 \pm 5$ & $58 \pm 2$ & $5.58 \pm 0.03$ \\
\hline $25 / 09 / 2011$ & 2455830.41 & $18900 \pm 1000$ & $2.45 \pm 0,05$ & 2.0 & $0.400 \pm 0,01$ & $700 \pm 70$ & $40 \pm 5$ & $55 \pm 2$ & $5.55 \pm 0.03$ \\
\hline $16 / 10 / 2011$ & 2455851.38 & $19100 \pm 1000$ & $2.50 \pm 0,05$ & 2.0 & $0.238 \pm 0,01$ & $350 \pm 35$ & $50 \pm 5$ & $53 \pm 2$ & $5.53 \pm 0.03$ \\
\hline $26 / 02 / 2012$ & 2455984.63 & $19000 \pm 1000$ & $2.50 \pm 0,05$ & 2.0 & $0.460 \pm 0,01$ & $600 \pm 60$ & $30 \pm 5$ & $52 \pm 2$ & $5.50 \pm 0.03$ \\
\hline $30 / 09 / 2012$ & 2456201.23 & $18600 \pm 1000$ & $2.30 \pm 0,05$ & 2.0 & $0.210 \pm 0,01$ & $230 \pm 25$ & $80 \pm 10$ & $65 \pm 2$ & $5.67 \pm 0.03$ \\
\hline
\end{tabular}




\begin{tabular}{|c|c|c|c|c|c|c|c|c|c|}
\hline 03/10/2012 & 2456204.26 & $18600 \pm 1000$ & $2.30 \pm 0,05$ & 2.0 & $0.200 \pm 0,02$ & $230 \pm 50$ & $30 \pm 5$ & $65 \pm 3$ & $5.67 \pm 0.06$ \\
\hline $14 / 10 / 2012$ & 2456215.28 & $19000 \pm 1000$ & $2.43 \pm 0,05$ & 2.0 & $0.293 \pm 0,01$ & $340 \pm 35$ & $30 \pm 5$ & $54 \pm 2$ & $5.54 \pm 0.03$ \\
\hline $14 / 11 / 2012$ & 2456246.24 & $19100 \pm 1000$ & $2.46 \pm 0,05$ & 2.0 & $0.200 \pm 0,02$ & $450 \pm 90$ & $70 \pm 5$ & $58 \pm 3$ & $5.61 \pm 0.06$ \\
\hline $16 / 03 / 2013$ & 2456368.52 & $19000 \pm 1000$ & $2.45 \pm 0,05$ & 2.0 & $0.300 \pm 0,01$ & $300 \pm 30$ & $40 \pm 5$ & $52 \pm 2$ & $5.51 \pm 0.03$ \\
\hline $21 / 04 / 2013$ & 2456404.47 & $19000 \pm 1000$ & $2.45 \pm 0,05$ & 2.0 & $0.305 \pm 0,01$ & $330 \pm 35$ & $40 \pm 5$ & $54 \pm 2$ & $5.54 \pm 0.03$ \\
\hline $25 / 04 / 2013$ & 2456408.47 & $19000 \pm 1000$ & $2.45 \pm 0,05$ & 2.0 & $0.305 \pm 0,01$ & $330 \pm 35$ & $30 \pm 5$ & $54 \pm 2$ & $5.54 \pm 0.03$ \\
\hline $19 / 07 / 2013$ & 2456493.37 & $19000 \pm 1000$ & $2.40 \pm 0,05$ & 2.0 & $0.295 \pm 0,01$ & $310 \pm 30$ & $35 \pm 5$ & $58 \pm 2$ & $5.60 \pm 0.03$ \\
\hline $20 / 07 / 2013$ & 2456494.35 & $18700 \pm 1000$ & $2.35 \pm 0,05$ & 2.0 & $0.200 \pm 0,01$ & $250 \pm 25$ & $50 \pm 5$ & $63 \pm 2$ & $5.65 \pm 0.03$ \\
\hline $21 / 07 / 2013$ & 2456495.33 & $18700 \pm 1000$ & $2.35 \pm 0,05$ & 2.0 & $0.200 \pm 0,01$ & $250 \pm 25$ & $65 \pm 5$ & $63 \pm 2$ & $5.65 \pm 0.03$ \\
\hline $22 / 07 / 2013$ & 2456496.35 & $18700 \pm 1000$ & $2.35 \pm 0,05$ & 2.0 & $0.200 \pm 0,01$ & $250 \pm 25$ & $110 \pm 10$ & $63 \pm 2$ & $5.65 \pm 0.03$ \\
\hline $23 / 07 / 2013$ & 2456497.52 & $18700 \pm 1000$ & $2.35 \pm 0,05$ & 2.0 & $0.200 \pm 0,01$ & $250 \pm 25$ & $80 \pm 10$ & $63 \pm 2$ & $5.65 \pm 0.03$ \\
\hline $25 / 07 / 2013$ & 2456499.55 & $18700 \pm 1000$ & $2.35 \pm 0,05$ & 2.0 & $0.200 \pm 0,02$ & $250 \pm 50$ & $150 \pm 15$ & $63 \pm 3$ & $5.65 \pm 0.06$ \\
\hline $30 / 07 / 2013$ & 2456504.35 & $18700 \pm 1000$ & $2.35 \pm 0,05$ & 2.0 & $0.200 \pm 0,01$ & $250 \pm 25$ & $55 \pm 5$ & $63 \pm 2$ & $5.65 \pm 0.03$ \\
\hline $01 / 08 / 2013$ & 2456506.57 & $18700 \pm 1000$ & $2.40 \pm 0,05$ & 1.6 & $0.215 \pm 0,01$ & $250 \pm 25$ & $40 \pm 5$ & $60 \pm 2$ & $5.60 \pm 0,03$ \\
\hline $02 / 08 / 2013$ & 2456507.34 & $19000 \pm 1000$ & $2.43 \pm 0,05$ & 2.0 & $0.295 \pm 0,01$ & $340 \pm 35$ & $80 \pm 10$ & $54 \pm 2$ & $5.54 \pm 0,03$ \\
\hline $03 / 08 / 2013$ & 2456508.34 & $19000 \pm 1000$ & $2.43 \pm 0,05$ & 2.0 & $0.295 \pm 0,01$ & $340 \pm 35$ & $70 \pm 5$ & $54 \pm 2$ & $5.54 \pm 0,03$ \\
\hline
\end{tabular}


Tabla 4.2. Parámetros estelares y del viento de 55 Cyg.

\begin{tabular}{|c|c|c|c|c|c|c|c|c|c|}
\hline & & & & & & & & & \\
\hline $\begin{array}{c}\text { Época } \\
\mathrm{dd} / \mathrm{mm} / \mathrm{aa}\end{array}$ & DJ & $\begin{array}{l}T_{\text {ef }} \\
{[\mathrm{K}]}\end{array}$ & $\begin{array}{l}\log g \\
{[\operatorname{cgs}]}\end{array}$ & $\beta$ & $\begin{array}{c}\dot{\mathrm{M}} \\
{\left[10^{-6} M_{\odot} / \mathrm{año}\right]}\end{array}$ & $\begin{array}{c}\mathrm{v}_{\infty} \\
{[\mathrm{km} / \mathrm{s}]}\end{array}$ & $\begin{array}{c}\mathrm{v}_{\operatorname{mac}}\left(\mathrm{H}_{\alpha}\right) \\
{[\mathrm{km} / \mathrm{s}]}\end{array}$ & $\begin{array}{c}\mathrm{R}_{\star} \\
{\left[\mathrm{R}_{\odot}\right]}\end{array}$ & $\log \mathrm{L} / \mathrm{L}_{\odot}$ \\
\hline $17 / 10 / 2013$ & 2456582.72 & $19000 \pm 1000$ & $2.50 \pm 0,05$ & 2.0 & $0.295 \pm 0,005$ & $310 \pm 30$ & $50 \pm 5$ & $58 \pm 1$ & $5.60 \pm 0,03$ \\
\hline $18 / 10 / 2013$ & 2456583.70 & $19000 \pm 1000$ & $2.50 \pm 0,05$ & 2.0 & $0.295 \pm 0,005$ & $310 \pm 30$ & $20 \pm 5$ & $58 \pm 1$ & $5.60 \pm 0,03$ \\
\hline $19 / 10 / 2013$ & 2456584.62 & $19000 \pm 1000$ & $2.50 \pm 0,05$ & 2.0 & $0.310 \pm 0,005$ & $320 \pm 30$ & $35 \pm 5$ & $58 \pm 1$ & $5.60 \pm 0,03$ \\
\hline $20 / 10 / 2013$ & 2456585.67 & $19000 \pm 1000$ & $2.47 \pm 0,05$ & 2.0 & $0.320 \pm 0,005$ & $320 \pm 30$ & $50 \pm 5$ & $57 \pm 1$ & $5.59 \pm 0,03$ \\
\hline $21 / 10 / 2013$ & 2456586.73 & $19100 \pm 1000$ & $2.50 \pm 0,05$ & 2.0 & $0.280 \pm 0,005$ & $300 \pm 30$ & $60 \pm 5$ & $57 \pm 1$ & $5.60 \pm 0,03$ \\
\hline $22 / 10 / 2013$ & 2456587.70 & $19000 \pm 1000$ & $2.40 \pm 0,05$ & 2.0 & $0.285 \pm 0,005$ & $310 \pm 30$ & $60 \pm 5$ & $58 \pm 1$ & $5.60 \pm 0,03$ \\
\hline $23 / 10 / 2013$ & 2456588.59 & $19000 \pm 1000$ & $2.40 \pm 0,05$ & 2.0 & $0.285 \pm 0,005$ & $350 \pm 35$ & $60 \pm 5$ & $58 \pm 1$ & $5.57 \pm 0,03$ \\
\hline $25 / 10 / 2013$ & 2456590.59 & $18650 \pm 1000$ & $2.40 \pm 0,05$ & 2.0 & $0.190 \pm 0,005$ & $300 \pm 30$ & $40 \pm 5$ & $58 \pm 1$ & $5.57 \pm 0,03$ \\
\hline $26 / 10 / 2013$ & 2456591.59 & $18600 \pm 1000$ & $2.40 \pm 0,05$ & 2.0 & $0.180 \pm 0,005$ & $270 \pm 30$ & $50 \pm 5$ & $57 \pm 1$ & $5.55 \pm 0,03$ \\
\hline $27 / 10 / 2013$ & 2456592.59 & $18570 \pm 1000$ & $2.40 \pm 0,05$ & 2.0 & $0.170 \pm 0,005$ & $270 \pm 30$ & $50 \pm 5$ & $56 \pm 1$ & $5.53 \pm 0,03$ \\
\hline $28 / 10 / 2013$ & 2456593.60 & $18570 \pm 1000$ & $2.40 \pm 0,05$ & 2.0 & $0.170 \pm 0,005$ & $270 \pm 30$ & $40 \pm 5$ & $56 \pm 1$ & $5.53 \pm 0,03$ \\
\hline $30 / 10 / 2013$ & 2456595.71 & $19000 \pm 1000$ & $2.36 \pm 0,05$ & 2.2 & $0.284 \pm 0,005$ & $330 \pm 30$ & $40 \pm 5$ & $57 \pm 1$ & $5.58 \pm 0,03$ \\
\hline $31 / 10 / 2013$ & 2456596.71 & $18600 \pm 1000$ & $2.40 \pm 0,05$ & 2.0 & $0.180 \pm 0,005$ & $270 \pm 30$ & $60 \pm 5$ & $57 \pm 1$ & $5.55 \pm 0,03$ \\
\hline $01 / 11 / 2013$ & 2456597.57 & $18600 \pm 1000$ & $2.40 \pm 0,05$ & 2.0 & $0.175 \pm 0,005$ & $260 \pm 30$ & $60 \pm 5$ & $57 \pm 1$ & $5.55 \pm 0,03$ \\
\hline $07 / 11 / 2013$ & 2456603.59 & $18570 \pm 1000$ & $2.40 \pm 0,05$ & 2.0 & $0.170 \pm 0,005$ & $270 \pm 30$ & $40 \pm 5$ & $56 \pm 1$ & $5.53 \pm 0,03$ \\
\hline
\end{tabular}




\subsection{Discusión}

Para investigar la relación entre los fenómenos de variabilidad observados es necesario contar con campañas de observación homogéneas y extendidas en el tiempo. Es así que para entender la variabilidad observada en las líneas fotosféricas de 55 Cyg se hizo un análisis de casi 400 espectros ópticos mediante el método de los momentos. Esto arrojó como resultado la existencia de 19 períodos (aunque probablemente hay más): 12 de estos períodos están en un rango de $\sim 2.7$ hs. a $\sim 24$ hs. y deben pertenecer a modos $\mathrm{p}$ de pulsación, y 6 están entre 1 y 6 días y podrían estar asociados a modos g. El período más largo encontrado es de 22.5 días y podría estar relacionado a los modos radiales extraños. Apoyando esta interpretación, el valor de L/M es del mismo orden que el asociado a modos extraños. La existencia de muchos modos cortos de pulsación puede influir en los perfiles fotosféricos, y quizá sean responsables de las incertezas tanto en la velocidad de rotación como en la macroturbulencia. Como el análisis de momentos de las líneas fue realizado por los otros coautores de nuestro artículo (Kraus et al. 2015), el desarrollo de este método y los resultados están explicados en el apéndice 6).

Ante todo hay que remarcar que los cambios en escalas de tiempo de un día en la forma e intensidad de las líneas no deben interpretarse como cambios reales en la pérdida de masa. Cada aumento de la densidad en la base del viento debido a eyecciones de masa (impulsadas por pulsaciones u otro tipo de inestabilidad) necesitaría más de un día para llegar a la región donde se forma $\mathrm{H} \alpha$.

Igualmente hay que mencionar tres ocasiones en las que $\mathrm{H} \alpha$ desaparece por completo (o casi), indicando en ese caso en particular que la emisión del viento fue compensada por una absorción fotosférica. La ausencia de la línea $\mathrm{H}_{\alpha}$ fue reportada por Maharramov 2013. el 2-4/9/2010 y nosotros detectamos lo mismo en nuestras observaciones del 24/7/2012 y 26/7/2013. Lo interesante es que luego de la reaparición del perfil, éste presentó una forma diferente, por ejemplo, una intensa emisión creció en intensidad entre el 10 y 12/8/2013, lo cual podría ser explicado por un aumento de materia en la región de formación de $\mathrm{H} \alpha$. Más aún, el comportamiento de la línea de HeI $\lambda 6678 \AA$ en el mismo período revela un fuerte incremento en la velocidad radial (panel intermedio en la fig. 6.9 con un salto en simultáneo del ancho equivalente (panel superior de la fig. 6.9.

Por otro lado, según los parámetros estelares que derivamos en este trabajo, éstos arrojan un valor de $\mathrm{L} / \mathrm{M} \sim 1.1 \times 10^{4} \mathrm{~L}_{\odot} / \mathrm{M}_{\odot}$, lo cual concuerda con la ocurrencia de modos radiales extraños Gautschy \& Glatzel 1990 Glatzel 1994 Saio et al. 1998. Las propiedades estelares de 55 Cyg son similares a aquellas de HD 50064, para la cual Aerts et al. 2010b argumentaron que la estrella estaría construyendo un envoltura circunestelar, mientras pulsa en un modo radial extraño. Un escenario similar podemos imaginarnos para 55 Cyg: la primer frecuencia, $\mathrm{f}_{1}$ (22.5 días), tiene la longitud adecuada para ser interpretada como un modo radial extraño como predice la teoría (Saio et al. 2013a. Este período quizá esté conectado con las variaciones intermedias observadas en $\mathrm{H}_{\alpha} \mathrm{y}$ en las tasas de pérdida de masa. Considerando las observaciones de Arizona obtenidas en octubre de 2013, parece ser que la tasa de pérdida de masa se mantiene más o menos constante a lo largo de poco más que una semana, seguida de un salto hacia un régimen menor de pérdida de masa. 
Otro período de al menos 5 días de comportamiento constante es reconocido en julio de 2013.

Pruebas adicionales para posibles conexiones entre pulsaciones y pérdida de masa son proporcionadas por la variabilidad en la forma de $\mathrm{H}_{\alpha}$ y por la extensión débil y superficial de las alas a $\pm 1200 \mathrm{~km} / \mathrm{s}$. Esta velocidad es mucho mayor que la velocidad terminal, por lo que estas alas no se estarían formando en el viento. En su lugar, nosotros interpretamos a esas alas como originadas por el scattering coherente de electrones en las capas de la envoltura extendida de la estrella por encima de la región de formación. Como estas alas no fueron reportadas por otros autores en trabajos previos (a excepción de (Ebbets 1982), y se mantuvieron estables durante los últimos 4 años, la actividad pulsacional podría haber conducido a una atmósfera ópticamente opaca en expansión. 


\section{Capítulo 5}

\section{Discusión general}

En este capítulo discutiremos en primer lugar la determinación de los parámetros estelares de las estrellas supergigantes B, comparando los mismos con resultados obtenidos por otros autores. También analizaremos los resultados preliminares de las mediciones de los anchos equivalentes del $\mathrm{C}, \mathrm{N}$ y $\mathrm{O}$ y dónde se ubican los diferentes objetos en las regiones de inestabilidad del diagrama HR.

La segunda parte estará dedicada a discutir la variabilidad de los vientos y su posible vinculación con las pulsaciones estelares como mecanismo extra a la radiación capaz de desencadenar también pérdida de masa en estos objetos.

\subsection{Parámetros estelares}

Los valores encontrados en la literatura que corresponden a diferentes estimaciones de $\mathrm{T}_{\text {ef }} \mathrm{y} \log \mathrm{g}$ presentan discrepancias de $\left|\Delta \mathrm{T}_{\text {ef }}\right|>1500 \mathrm{~K}, \mathrm{y}|\Delta \log \mathrm{g}| \sim 0.2$ dex con algunas de las estrellas estudiadas en este trabajo (ver tabla 6.1 del apéndice). Es importante resaltar que los valores de las $\mathrm{T}_{\text {ef }}$ fueron derivadas usando diferentes métodos o aproximaciones. Los métodos más comunes usados fueron el ajuste de la SED y el ajuste de perfiles sintéticos a los perfiles de línea observados. Para este último método, existen varios códigos de cálculo que resuelven el transporte de radiación empleando diferentes geometrías para la atmósfera y condiciones de equilibrio (LTE y NLTE), distintas aproximaciones de los coeficientes de absorción, etc.. Si bien los usuarios buscan reproducir las mismas líneas de Si y He para determinar los balances de ionización, y a partir de allí la temperatura efectiva, los resultados podrían ser diferentes, debido a las hipótesis adoptadas. Los códigos más conocidos son, por ejemplo, TLUSTY + SYNSPEC (Hubeny \& Lanz 1995b Lanz \& Hubeny 2007) Hubeny \& Lanz 2017), CMFGEN (Hillier \& Miller 1998) y FASTWIND (Santolaya-Rey et al. 1997 Puls et al. 2005). Estos dos últimos resuelven el transporte radiativo en NLTE teniendo en cuenta los efectos del viento y blanketing.

Sin embargo, para la mayoría de las estrellas de nuestra muestra hemos encontrado $\mathrm{T}_{\text {ef }}$ que presentan un buen acuerdo con los valores estimados por Crowther et al. 2006a, siete estrellas en común, incluida 55 Cyg), Lefever et al. 2007. 8 estrellas en común), Searle 
et al. 2008a, seis estrellas en común, incluida 55 Cyg) y Fraser et al. 2010, 12 estrellas en común) Entre todos estos autores, las mayores discrepancias en $\mathrm{T}_{\text {ef }}$ las encontramos para 6 estrellas: HD 42087, HD 52089, HD 53138, HD 64740, HD 92964 y HD 99953, que muestran apreciables diferencias $\left(\Delta T_{\text {ef }}>2000 \mathrm{~K}\right)$. Casualmente cuatro de ellas presentan características peculiares; HD 53138 y HD 64740 muestran variaciones en las líneas UV (ver detalles en Prinja et al. 2002, atribuidos a cambios en la ionización del viento. Por otro lado, la estrella HD 52089 presenta un campo magnético variable Morel et al. 2008 y emisión en rayos-X (Kholtygin et al. 2015), y al igual que HD 99953, está localizada dentro de la región de biestabilidad, lo que podría sugerir que estas estrellas muestran variaciones intrínsecas de temperatura.

Con respecto a la velocidad de macroturbulencia, nosotros esperábamos encontrar una relación lineal con la $T_{\text {ef }}$ como fue reportado por Markova \& Puls 2008) y Fraser et al. 2010. Estos autores encuentran que $\mathrm{v}_{\text {macro }}$ tiene valores de $\simeq 60 \mathrm{~km} / \mathrm{s}$ para el tipo espectral B0 y de $\simeq 30 \mathrm{~km} / \mathrm{s}$ para el tipo espectral B9, y que la relación presenta mucha dispersión (ver cap.2 fig. 2.5). En este trabajo no podemos confirmar esta relación porque encontramos que para ajustar las líneas fotosféricas fue necesario utilizar valores grandes de $\mathrm{v}_{\text {macro }}$ para todas las estrellas (alrededor de $50-60 \mathrm{~km} / \mathrm{s}$ ). La falta de correlación de nuestros valores con la $T_{\text {ef }}$ puede deberse a la baja resolución espectral de nuestras observaciones ( $R$ $\sim$ 13000), que no es adecuada para utilizar el método de la Transformada de Fourier por lo que tuvimos que utilizar valores de vsin i tomados de la literatura. Y como explicamos en el cap. 2, v sin i y $\mathrm{v}_{\text {macro }}$ son dos cantidades que están fuertemente vinculadas.

Por último, la mayor discrepancia encontrada en los parámetros estelares, con respecto a trabajos previos, está relacionada con la determinación del radio estelar y surge de los diferentes métodos usados para determinarlo y de la incerteza que existe en las mediciones de las distancias a las estrellas. Contrariamente a otros autores, como explicamos en el cap. 3, hemos adoptado un valor medio para el radio estelar después de haberlo calculado mediante varios métodos. Es por eso que pensamos que los valores obtenidos de $R_{\star}$ en este trabajo son confiables.

\subsection{Composición química}

Otro parámetro estelar importante es la composición química en la superficie de la estrella, ya que éste es un indicador del estado evolutivo de la misma, y es relevante en el modelado de la estructura estelar, el cálculo de los procesos de convección y pérdida de masa. Quizás este parámetro es uno de los que mayor controversia genera, ya que los modelos de evolución estelar no logran predecir los valores observados, sobre todo en lo referente a las abundancias de He, C, N y O observadas en las estrellas BSGs Saio et al. 2013b. Cabe destacar que todos los modelos presentados en los caps. 3 y 4 fueron calculados empleando una abundancia tipo solar, por lo que nos preguntamos qué efectos tendrían diferentes abundancias químicas sobre la determinación de los parámetros estelares y la pérdida de masa. 


\subsubsection{Abundancia de helio}

Para discutir el contenido de He en nuestra muestra de estrellas, analizamos el comportamiento de las líneas de $\mathrm{He}$ e $\mathrm{H}$ al considerar distintas abundancias de $\mathrm{N}(\mathrm{He}) / \mathrm{N}(\mathrm{H})$ y discutimos los efectos de este cambio en la determinación de la temperatura efectiva y la pérdida de masa.

Como es sabido, ante condiciones de muy alta densidad se observan líneas de intercombinación de He como consecuencia de la ruptura de la regla de selección del dipolo en presencia de una perturbación del campo eléctrico. Por ejemplo, la transición de He I $\lambda 4470 \AA\left(2^{3} \mathrm{P}-4^{3} \mathrm{~F}\right)$, se observa superpuesta al ala azul de la transición permitida de la línea He I $\lambda 4471 \AA$. Entonces es de esperar que un aumento en la abundancia de He altere las intensidades de las líneas de He, tanto prohibidas como permitidas.

Para discutir el efecto de la abundancia de He en los perfiles de líneas calculamos distintos modelos empleando el código FASTWIND, considerando una tasa de pérdida de masa constante $\left(1.9 \times 10^{-7} \mathrm{M}_{\odot} /\right.$ año$)$. Este resultado se muestra en la fig. 5.1, donde se observa que al incrementar la abundancia relativa de $\mathrm{N}(\mathrm{He}) / \mathrm{N}(\mathrm{H})$ de 0.1 a 0.4 , se intensifican todas las líneas, en particular, las componentes prohibidas asociadas a las transiciones He I $\lambda 4471 \AA$ y HeI $\lambda 4923 \AA$. De esta manera, estas componentes desarrollan una prominente absorción en el ala azul de las líneas permitidas mencionadas que se manifiestan como pronunciadas asimetrías en los perfiles, efecto que se observa incluso si la velocidad de rotación proyectada de la estrella es tan alta como $\sim 60 \mathrm{~km} / \mathrm{s}$ (ver fig. 5.1). Así, la presencia e intensidad de las componentes prohibidas es de gran ayuda para determinar abundancias de He superficial. Como en nuestros espectros esta componente no se observa, concluimos que no es necesario aumentar la abundancia de He en los modelos, y por lo tanto adoptamos una abundancia solar de He para modelar los perfiles de línea.

Por otro lado, es sabido que la línea $\mathrm{H}_{\alpha}$ es también sensible a las variaciones del contenido de He, y en este sentido es que los modelos de vientos ricos en He producen anchos equivalentes en $\mathrm{H}_{\alpha}$ que son un factor 2-3 más chicos que aquellos calculados con abundancia solar (Petrov et al. 2014). A fin de analizar el comportamiento de la tasa de pérdida de masa, calculamos perfiles de $\mathrm{H}_{\alpha}$ variando los valores de la abundancia relativa $\mathrm{N}(\mathrm{He}) / \mathrm{N}(\mathrm{H})$ entre $0.1,0.2$ y 0.4 y manteniendo constante el valor de $\dot{\mathrm{M}}\left(1.9 \times 10^{-7} \mathrm{M}_{\odot} /\right.$ año $)$. El resultado se muestra en el panel superior de la fig. 5.2, donde se ve claramente cómo disminuye la intensidad de la componente en emisión del perfil de $\mathrm{H}_{\alpha}$ a medida que la abundancia de He aumenta. Luego, para poder reproducir nuevamente el perfil de $\mathrm{H}_{\alpha}$ calculado anteriormente para el modelo con $\mathrm{N}(\mathrm{He}) / \mathrm{N}(\mathrm{H})=0.1$ y $\dot{\mathrm{M}}=1.9 \times 10^{-7} \mathrm{M}_{\odot} /$ año, pero empleando abundancias relativas mayores de He, debemos aumentar la pérdida de masa. Encontramos que obtenemos prácticamente los mismos perfiles si $\dot{M}=2.5 \times 10^{-7} \mathrm{M}_{\odot} /$ año y $3.5 \times 10^{-7}$ $\mathrm{M}_{\odot} /$ año para $\mathrm{N}(\mathrm{H}) / \mathrm{N}(\mathrm{He})=0.2$ y 0.4 , respectivamente (panel inferior de la fig. 5.2 .

Es conveniente resaltar la falta de unicidad de los modelos con la composición química si se modela únicamente la línea de $\mathrm{H}_{\alpha}$. Por eso es importante acompañar el análisis modelando también las líneas de He, especialmente las componentes prohibidas, en combinación con otros elementos químicos dado que un cambio en la abundancia de He también podría generar cambios en la $\mathrm{T}_{\text {ef }}$. 

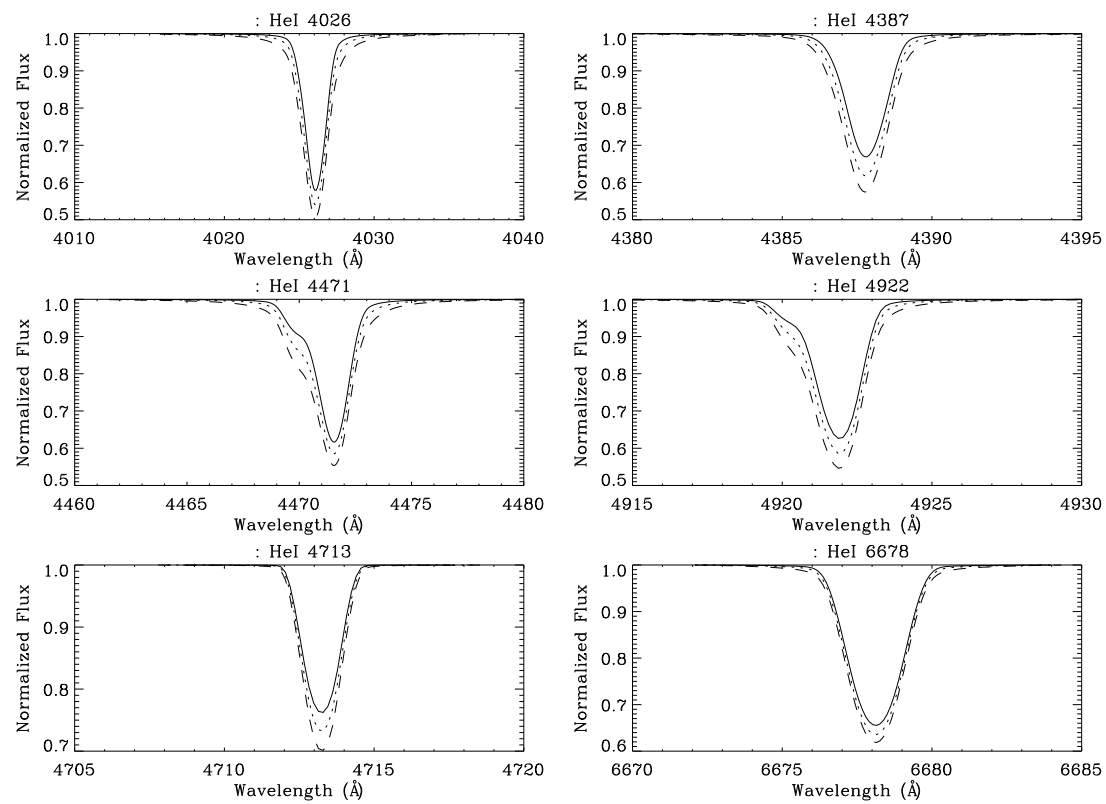

Figura 5.1. Variación de la intensidad de las líneas de He con la abundancia química. En cada panel se representan las diferentes líneas de He calculadas con $\mathrm{N}(\mathrm{He}) / \mathrm{N}(\mathrm{H})=0.1$ (línea sólida), $\mathrm{N}(\mathrm{He}) / \mathrm{N}(\mathrm{H})=0.2$ (línea con puntos) y $\mathrm{N}(\mathrm{He}) / \mathrm{N}(\mathrm{H})=0.4$ (línea con rayas). Las intensidades de las componentes prohibidas de las líneas He I $\lambda 4470 \AA$ y He I $\lambda 4922 \AA$ sirven como indicadores ideales para cuantificar el contenido de He en la superficie de la estrella.
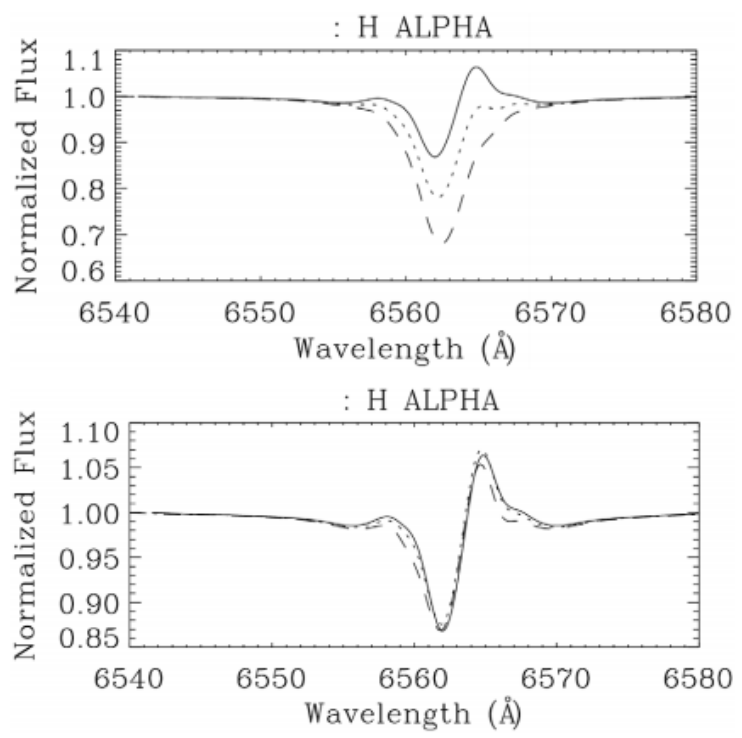

Figura 5.2. Arriba: variaciones en el ancho equivalente de la línea $\mathrm{H}_{\alpha}$ para diferentes abundancias de He: $\mathrm{N}(\mathrm{He}) / \mathrm{N}(\mathrm{H})=0.1$ (línea sólida), $\mathrm{N}(\mathrm{He}) / \mathrm{N}(\mathrm{H})=0.2$ (línea con puntos) y $\mathrm{N}(\mathrm{He}) / \mathrm{N}(\mathrm{H})=0.4$ (línea con rayas), suponiendo que la pérdida de masa es constante. Abajo: perfiles de $\mathrm{H}_{\alpha}$ similares usando los mismos valores $\mathrm{N}(\mathrm{He}) / \mathrm{N}(\mathrm{H})$ que el gráfico superior pero con diferentes valores de $\dot{\mathrm{M}}$. 


\subsubsection{Abundancia de silicio}

En relación al modelado de las líneas de Si II y Si III, pudimos también obtener buenos ajustes usando la abundancia solar, pero la pregunta recae en cuán sensibles son las líneas de Si a los cambios de este contenido. La influencia de una metalicidad no solar en la $\mathrm{T}_{\text {ef }}$ fue discutida en detalle por Lefever et al. 2007) y Markova \& Puls 2008), quienes encontraron que valores altos de la abundancia de $\mathrm{Si}$ conducen a valores bajos de $\mathrm{T}_{\text {ef }}$, y viceversa. Por otro lado, un cambio en la temperatura efectiva $\left(\Delta \mathrm{T}_{\text {ef }}>1000 \mathrm{~K}\right)$ afectaría también a la intensidad de las líneas de He. Sin embargo, a partir del modelado en forma conjunta del balance de ionización de Si y He, no encontramos diferencias entre las temperaturas derivadas a partir de las líneas de estos elementos al considerar una abundancia solar. Por este motivo, la suposición de una abundancia tipo solar para ambos elemento: $\mathbb{1}^{1}$ está bien justificada. Resultados similares han sido obtenidos por Markova \& Puls 2008.

\subsubsection{Abundancia de CNO}

El estudio de las abundancias superficiales de CNO es importante para discutir la historia evolutiva de las estrellas y los efectos de la metalicidad en los parámetros del viento, como ya mencionamos en el cap. 2, secc 2.4.6.

Desde los años 70 numerosos trabajos han reportado anomalías en las líneas de CNO (ver referencias en Walborn 1976). Particularmente Walborn 1971) encontró una dicotomía de $\mathrm{N}$ y $\mathrm{C}$ que parece existir entre un cierto número de estrellas $\mathrm{O}$ y $\mathrm{B}$ y propuso una nueva clasificación análoga a la que existe para las estrellas WR. Los objetos con N intensificado y débil en $\mathrm{C}$ y/u $\mathrm{O}$ relativo a las estrellas normales para el mismo tipo espectral son clasificados como $\mathrm{ON}$ o BN, mientras que los espectros con deficiencia en $\mathrm{N}$ y evidencia de intensificaciones de $\mathrm{C}$, son clasificados como OC o BC.

La versión de FASTWIND que utilizamos no tiene implementado el modelado de las líneas de $\mathrm{C}, \mathrm{N}$ y $\mathrm{O}$, por lo que la determinación de abundancias químicas a partir del modelado de los perfiles de líneas de estos elementos no pudo realizarse. Por lo tanto, los resultados que presentamos aquí son preliminares y están basados en las mediciones de anchos equivalentes (EW).

Para discutir posibles incrementos en las abundancias de CNO, hemos medido los anchos equivalentes de las líneas de C II $\lambda \lambda 6578.05,6582.88 \AA$, N II $\lambda 4630 \AA$, N II $\lambda 4447 \AA$, O II $\lambda 4661 \AA$ y O II $\lambda 4596 \AA$ de casi todas las estrellas de nuestra muestra. Luego comparamos estos resultados con los obtenidos teóricamente por McErlean et al. 1999a con el código TLUSTY usando abundancias solares. Si bien estos modelos tienen ciertas limitaciones, nos sirven para poder identificar subgrupos de estrellas en diferentes estados evolutivos. Estas comparaciones están representadas en las figs. 5.3. 5.4 y 5.5.

Dentro de las estrellas de tipo espectral temprano, encontramos que HD 38771, HD 52382, HD 52089 y HD 115842 podrían modelarse con abundancias de CNO tipo solar ya que las cuatro estrellas muestran un comportamiento similar a las predicciones teóricas hechas

\footnotetext{
${ }^{1}$ Adoptamos $\mathrm{Y}_{\mathrm{He}}=0.1$, con $\mathrm{Y}_{\mathrm{He}}=\mathrm{N}(\mathrm{He}) / \mathrm{N}(\mathrm{H})$, y $\log (\mathrm{Si} / \mathrm{H})=-4.45 \pm 0.15$ dex, datos obtenidos de Grevesse \& Sauval 1998.
} 


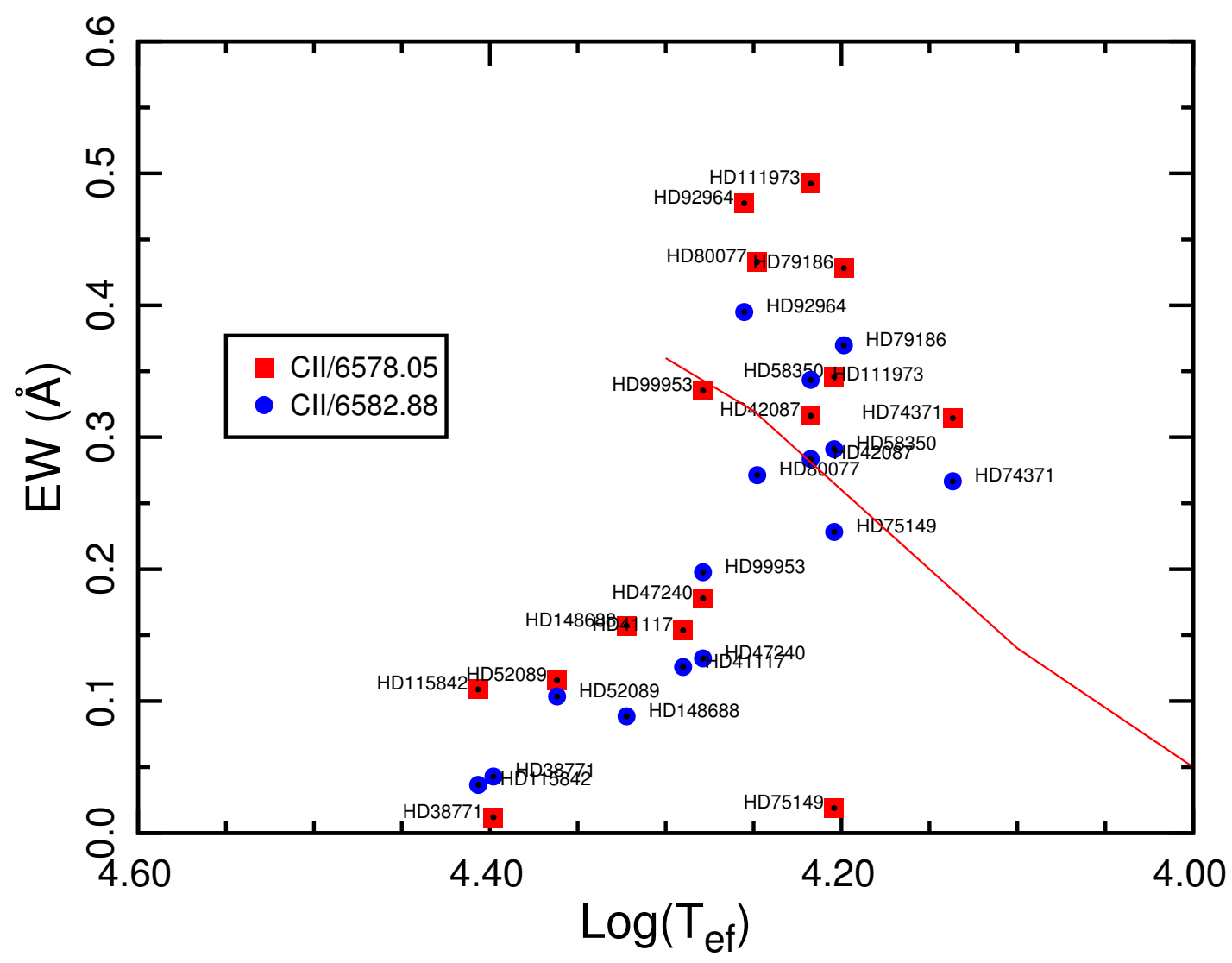

Figura 5.3. Anchos equivalentes observados del doblete de C II $\lambda 6578.05 \AA$ (cuadrados rojos) y C II $\lambda 6582.88 \AA$ (círculos azules) para el conjunto de las estrellas BSGs (con la excepción de $55 \mathrm{Cyg}$ ) presentadas en este trabajo. La curva roja corresponde a los cálculos NLTE realizados por McErlean et al. 1999a para una abundancia $[\mathrm{C} / \mathrm{H}]=8.20$ dex y vmicro $=10 \mathrm{~km} / \mathrm{s}$. 


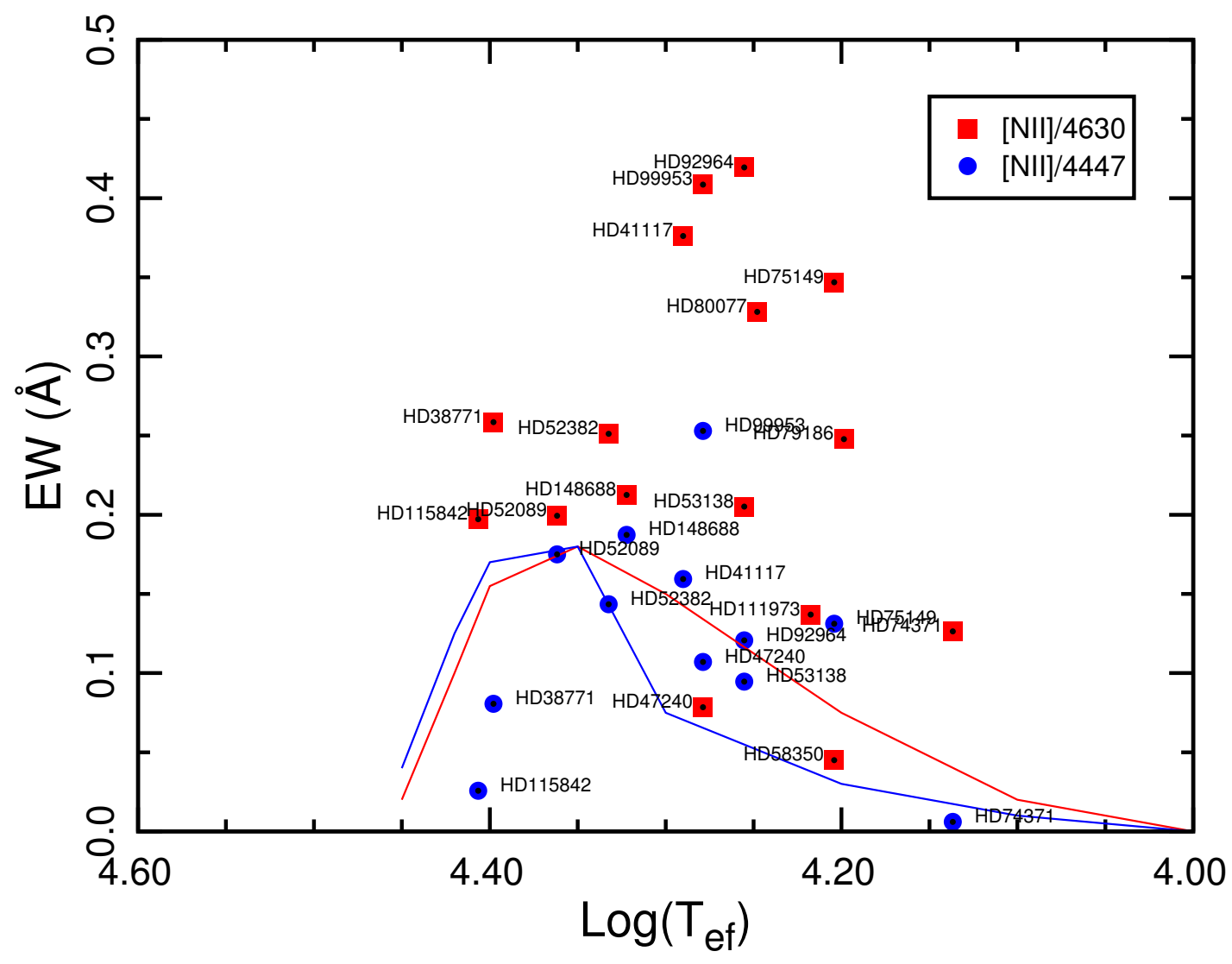

Figura 5.4. Anchos equivalentes observados en las líneas de N II $\lambda 4630 \AA$ (cuadrados rojos) y N II $\lambda 4447 \AA$ (círculos azules) para el conjunto de las estrellas BSGs (con la excepción de 55 Cyg) presentadas en este trabajo. La curva roja y azul corresponden a los cálculos NLTE realizados por McErlean et al. 1999a para las mismas líneas, utilizando una abundancia $[\mathrm{N} / \mathrm{H}]=7.69$ dex y $\mathrm{v}_{\text {micro }}=10 \mathrm{~km} / \mathrm{s}$. 


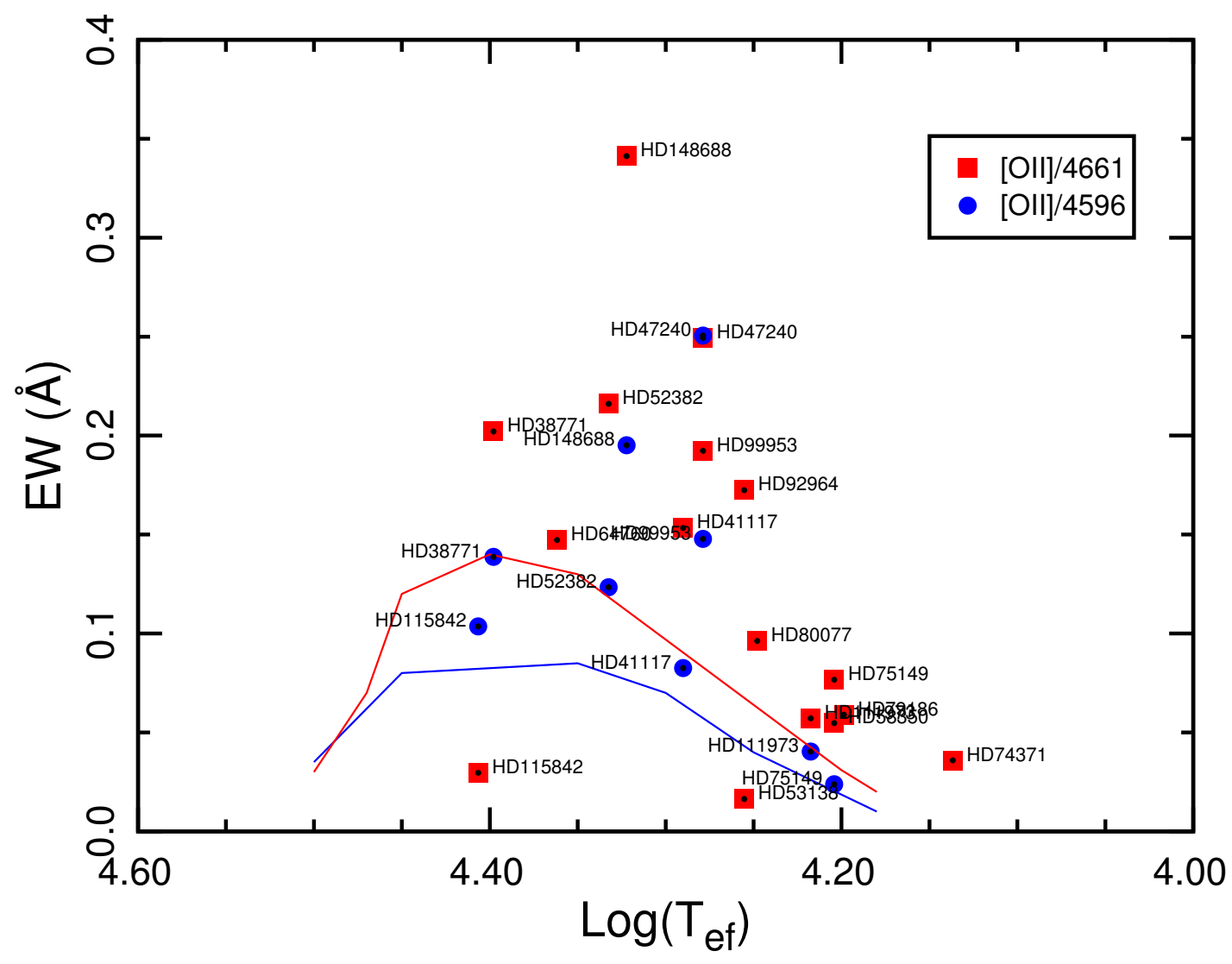

Figura 5.5. Anchos equivalentes observados en las líneas de O II $\lambda 4661 \AA$ (cuadrados rojos) y O II $\lambda 4596 \AA$ (círculos azules) para el conjunto de las estrellas BSGs (con la excepción de $55 \mathrm{Cyg}$ ) presentadas en este trabajo. La curvas roja y azul corresponden a los cálculos NLTE realizados por McErlean et al. 1999a para las mismas líneas utilizando una abundancia $[\mathrm{O} / \mathrm{H}]=8.55$ dex y $\mathrm{v}_{\text {micro }}=10 \mathrm{~km} / \mathrm{s}$. 
por McErlean et al. 1999a. En particular HD 38771 y HD 52382 muestran las líneas de C II muy débiles. Las otras estrellas de tipo espectral temprano, HD 47240 y HD 148688, presentan un gran exceso en el ancho equivalente de O II respecto al observado en el resto de las estrellas (ver fig. 5.5).

En lo que respecta a las estrellas de tipo espectral medio y tardío, éstas son las que presentan los comportamientos más interesantes. Por un lado están HD 41117 y HD 99953 con sobreintensificaciones en los EWs de las líneas de N II (ver fig. 5.4) y no así en los EWs de las líneas de CII. Y por otro lado encontramos cuatro estrellas con valores de EWs muy altos en CII (HD 53138, HD 58350, HD 74371 y HD 111973) y no así en N II. También identificamos un tercer grupo de estrellas cuyos EWs son muy altos tanto en C II como en N II (HD 92964, HD 80077 y HD 791869), sugiriendo la presencia de elementos moderadamente procesados en la superficie. Por último observamos que HD 75149 presenta uno de los EWs más altos en N II pero el comportamiento de las líneas de C II es ambiguo.

Walborn 1976 reportó que HD 38771 es una estrella normal y, por lo tanto, podemos suponer que HD 52382 también es una estrella normal, ya que los comportamientos de los EWs de CNO son muy similares a los de HD 38771. Walborn 1976 también propuso que HD 148688 es normal, y nosotros observamos que su comportamiento en los EWs de CNO es similar a los de HD 47240, por lo que podríamos pensar que esta última también es una estrella normal. En cambio, las demás estrellas de nuestra muestra presentan intensificado un sólo elemento mientras que los otros se comportan aparentemente de manera normal. Así, clasificamos a HD 41117 y HD 99953 como ricas en N (BRN, y quizá también HD 75149), mientras que HD 53138, HD 58350, HD 74371 y HD 111973 serían estrellas ricas en C (BRC). Resaltamos que Searle et al. 2008a mencionan que HD 53138 puede ser del tipo BN o BC, aunque Walborn (1976) la reportó como una estrella normal. También sugerimos que HD 92964, HD 80077 y HD 791869 presentarían elementos moderadamente procesados (MP) debido a los grandes valores que presentan los EW de las líneas de $\mathrm{C}$ y N, mientras que las estrellas HD 38771, HD 52089 y HD 115842 tendrían un comportamiento normal de CNO para sus tipos espectrales. Cabe mencionar que las estrellas que nosotros sugerimos como "normales", según mediciones de abundancias realizadas por Crowther et al. 2006a y Searle et al. 2008a todas poseen una sobreabundancia de N.

Otra manera de comparar el contenido relativo de CN es expresar el cociente del ancho equivalente de las líneas más intensas que hemos analizado en función de la temperatura efectiva. Estas dos líneas son C II 6578.05 Å y N II 4630 Å y el resultado se muestra en la fig. 5.6 .

Resulta interesante en este gráfico que las estrellas propuestas como BRC tienen un cociente de EWs mayor a 2. En este grupo encontramos también a la estrella HD 47240, para la cual el análisis previo sugería una clasificación normal. También se puede ver que las tres estrellas propuestas con alto contenido de $\mathrm{C}$ y N tienen cocientes de EW(C II 6578.05 $\AA) / \mathrm{EW}(\mathrm{N}$ II $4630 \AA$ ) entre 1 y 2. Dentro de las estrellas con EW(C II $6578.05 \AA$ )/EW(N II $4630 \AA)<0.5$ encontramos a las propuestas anteriormente como "normales" y BRN, lo que podría sugerir que todas estas estrellas poseen un moderado enriquecimiento de N. Esto concuerda con los trabajos anteriores realizados por Crowther et al. 2006a y Searle et al. 2008a donde reportan que todas las estrellas BSGs presentan sobreabundancias de N en 


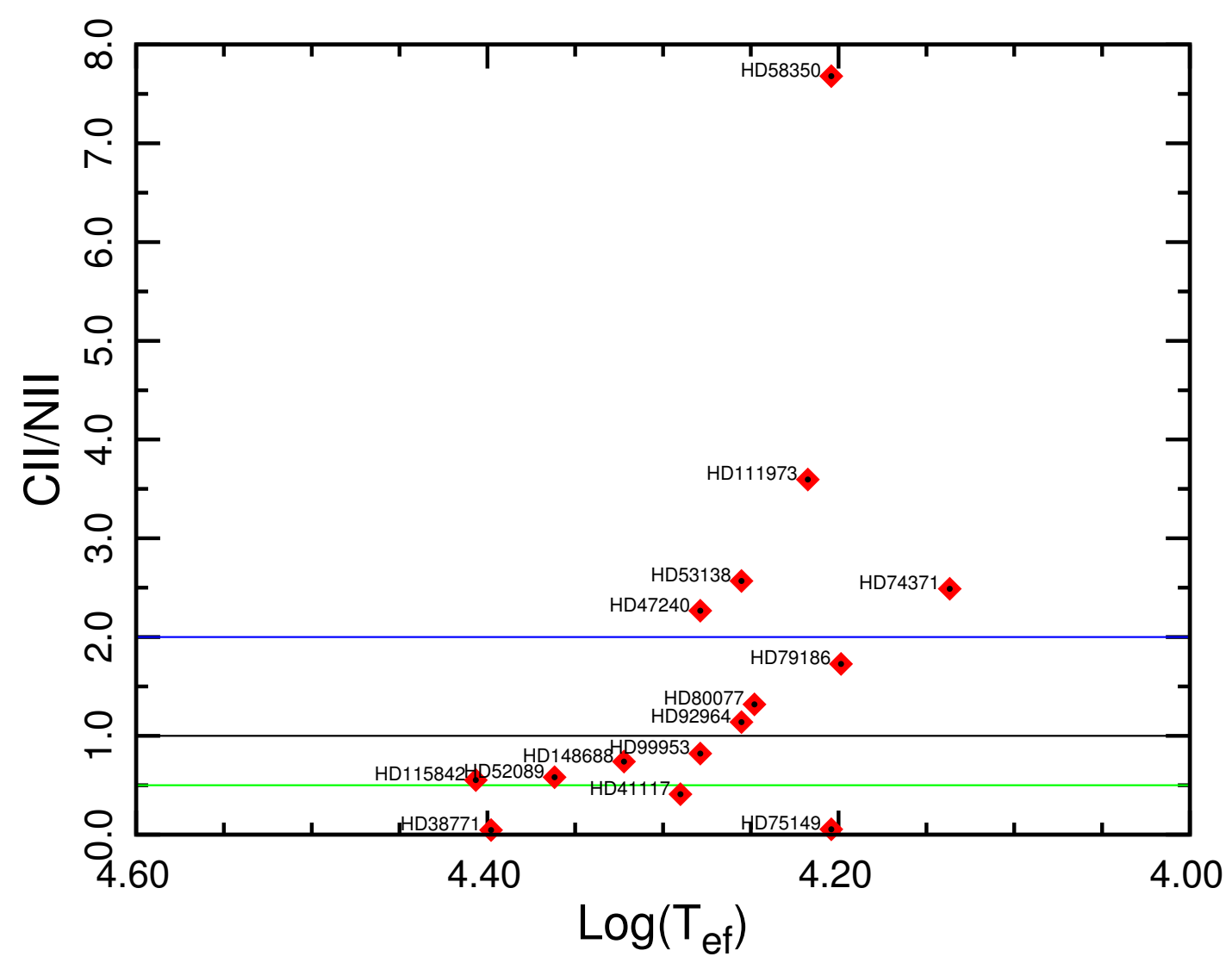

Figura 5.6. Cociente de anchos equivalentes observados de C II $6578.05 \AA$ y N II $4630 \AA$ en función de la temperatura efectiva 
Tabla 5.1. Clasificación tentativa de estrellas BSGs según la intensidad de las líneas de C y N.

\begin{tabular}{cccccc}
\hline \hline Estrella & TE & MP & BRN & BRC & Normal \\
\hline \hline HD 38 771 & B0.5 Ia & $\mid$ & $\mid$ & $\mid$ & X \\
HD 41 117 & B2 Ia & $\mid$ & X & $\mid$ & $\mid$ \\
HD 42 087 & B4 Ia & $\mid$ & $\mid$ & $\mid$ & X? \\
HD 47 240 & B1 Ib & $\mid$ & $\mid$ & X? & X \\
HD 52 089 & B1.5 II & $\mid$ & $\mid$ & $\mid$ & X \\
HD 52 382 & B1 Ia & $\mid$ & $\mid$ & X \\
HD 53 138 & B3 Ia & $\mid$ & $\mid$ & X & $\mid$ \\
HD 58 350 & B5 Ia & $\mid$ & X & $\mid$ \\
HD 64 760 & B0.5 Ib & $\mid$ & $\mid$ & X? \\
HD 74 371 & B6 Iab/b & $\mid$ & X & $\mid$ \\
HD 75 149 & B3 Ia & $\mid$ & X? & $\mid$ & $\mid$ \\
HD 79 186 & B5 Ia & X & $\mid$ & $\mid$ & $\mid$ \\
HD 80 077 & B2Ia+e & X & $\mid$ & $\mid$ & $\mid$ \\
HD 92 964 & B2.5Ia & X & $\mid$ & $\mid$ & $\mid$ \\
HD 99 953 & B1/2 Iab/b & X & $\mid$ & $\mid$ \\
HD 111 1973 & B2/3 Ia & $\mid$ & $\mid$ & X & $\mid$ \\
HD 115842 & B0.5 Ia/ab & $\mid$ & $\mid$ & X \\
HD 148 688 & B1 Iaeqp & $\mid$ & $\mid$ & X \\
\hline \hline
\end{tabular}

$\mathrm{MP} \equiv$ materia Moderadamente Procesada, $\mathrm{BRN} \equiv$ ricas en $\mathrm{N}, \mathrm{BRC} \equiv$ ricas en $\mathrm{C}$ y Normal $\equiv$ abundancias de CNO solares o levemente procesadas.

mayor o menor medida.

Nuestro esquema de clasificación queda resumido en la tabla 5.1 . donde puede observarse que las estrellas clasificadas como BRN tienen tipos espectrales entre B1 y B2. Este resultado coincide con la observación de abundancias realizada por Searle et al. (2008a) quienes reportan que en general se observa $\mathrm{N}$ enriquecido en las estrellas BSGs de menos temperatura efectiva y que el mayor enriquecimiento es visto entre los tipos espectrales B1 y B2. Nuesta clasificación también sugiere que las estrellas BRC están entre los tipos espectrales B3 y B6.

\subsection{Estado evolutivo}

En esta sección analizaremos la posición de las estrellas en el diagrama HR y discutiremos las posibles etapas evolutivas en las que éstas se encuentran. La figura 5.7 muestra dichas posiciones. En este diagrama se identifican además las regiones de inestabilidad para los diferentes modos de pulsación definidos por Saio 2011). La región de inestabilidad para modos p radiales de bajo orden está delimitada por las curvas azules: una columna vertical alrededor de $\log \mathrm{T}_{\mathrm{ef}} \sim 4.1$ (donde se encuentran las variables $\beta$ Cep; Saio et al.|2013b), otra alrededor de $\log \mathrm{T}_{\text {ef }} \sim 3.8$, y la curva horizontal en $\log \mathrm{L} / \mathrm{L}_{\odot} \sim 5.5$ que se extiende entre el rango de temperatura mencionado. Esta curva delimita el borde inferior de la región de 
inestabilidad de los modos extraños, que ocurren cuando $\mathrm{L} / \mathrm{M}>4$. Los modos g no-radiales (de bajo grado y alto orden) pueden ser excitados en la región delimitada por las curvas rojas: la región más pequeña tiene asociada un número $l=1$ y la más grande $l=2$. Por otro lado, los modos monótonamente inestables existen por encima de la curva magenta en la parte más luminosa del diagrama HR (donde se ubica la estrella LBV HD 80077). En el diagrama HR también están indicadas, con curvas color celeste, las regiones asociadas con la presencia de modos de oscilación convectivos para $\mathrm{l}=1$ (región pequeña) y $\mathrm{l}=2$ (región grande). En este diagrama también se muestran los caminos evolutivos (sin rotación) para diferentes masas iniciales, tomados de Ekström et al. 2012).

Es interesante resaltar la ubicación de los objetos que hemos estudiado en el diagrama de la fig. 5.7. HD 38771, HD 52089 y HD 115842 se ubican cerca de la TAMS y en la misma región de inestabilidad, donde los modos de pulsación p radiales están excitados (región limitada por las curvas azules). En esta misma región del diagrama HR se encuentra la estrella HD 64760 2 , con abundancias de CNO similares a las estrellas mencionadas anteriormente, según las determinaciones realizadas por Searle et al. 2008a y Crowther et al. 2006a. Considerando el análisis de los EWs realizados en la sección anterior donde estos objetos aparentan mostrar abundancias de CNO normales, podríamos concluir que estos objetos se encuentran en etapas finales de la secuencia principal o acaban de abandonarla. En particular, HD 52089 y HD 64760 tienen $\log \mathrm{L} / \mathrm{L}_{\odot}<4.6, \log \mathrm{L} / \mathrm{M}<4$ y se encuentran ubicadas en la región de inestabilidad correspondiente a las variables $\beta$ Cep, encontrada por Saio et al. 2013b y Georgy et al. 2014. Cabe mencionar que las estrellas $\beta$ Cep presentan mayoritariamente abundancias normales de CNO Walborn 1971.

Por otro lado, las otras tres estrellas BSGs de tipos espectrales tempranos (HD 47240, HD 52382 y HD 148688), clasificadas por nosotros como estrellas normales, ocupan otra región en el diagrama HR (fig. 5.7) que corresponde a la región de inestabilidad donde los modos no-radiales están excitados (región encerrada por las curvas rojas). Esto podría deberse a la presencia de elementos levemente procesados.

De estos resultados, podemos inferir que existen dos subgrupos de estrellas BSGs de tipo espectral temprano. Uno de ellos se ubica inmediatamente después de la TAMS, y otro grupo que parecería estar en una etapa evolutiva posterior, manifestando propiedades pulsacionales diferentes.

HD 41117 y HD 99953 son las únicas que presentan sobreintensificaciones claras en los EW de las líneas de N II (ver fig. 5.4) y que están ubicadas junto con algunas estrellas BSGs tempranas en la región donde lo modos no-radiales estarían excitados. Esto podría indicar que estos dos objetos son más evolucionados que las estrellas ubicadas en la misma región, propuestas como normales.

En el diagrama HR también se puede ver que el resto de las estrellas B de tipos espectrales medios y tardíos (símbolos verdes y rojos, respectivamente), con la excepción de HD 80077, están ubicadas en la región donde los modos convectivos oscilatorios deberían ser observados (curvas cian). Si bien la mayoría de estas BSGs presentan signos de sobreabun-

\footnotetext{
${ }^{2}$ No nos fue posible determinar los EW de las líneas de CNO en HD 64760 dado que son poco intensas a causa de su alta rotación estelar.
} 


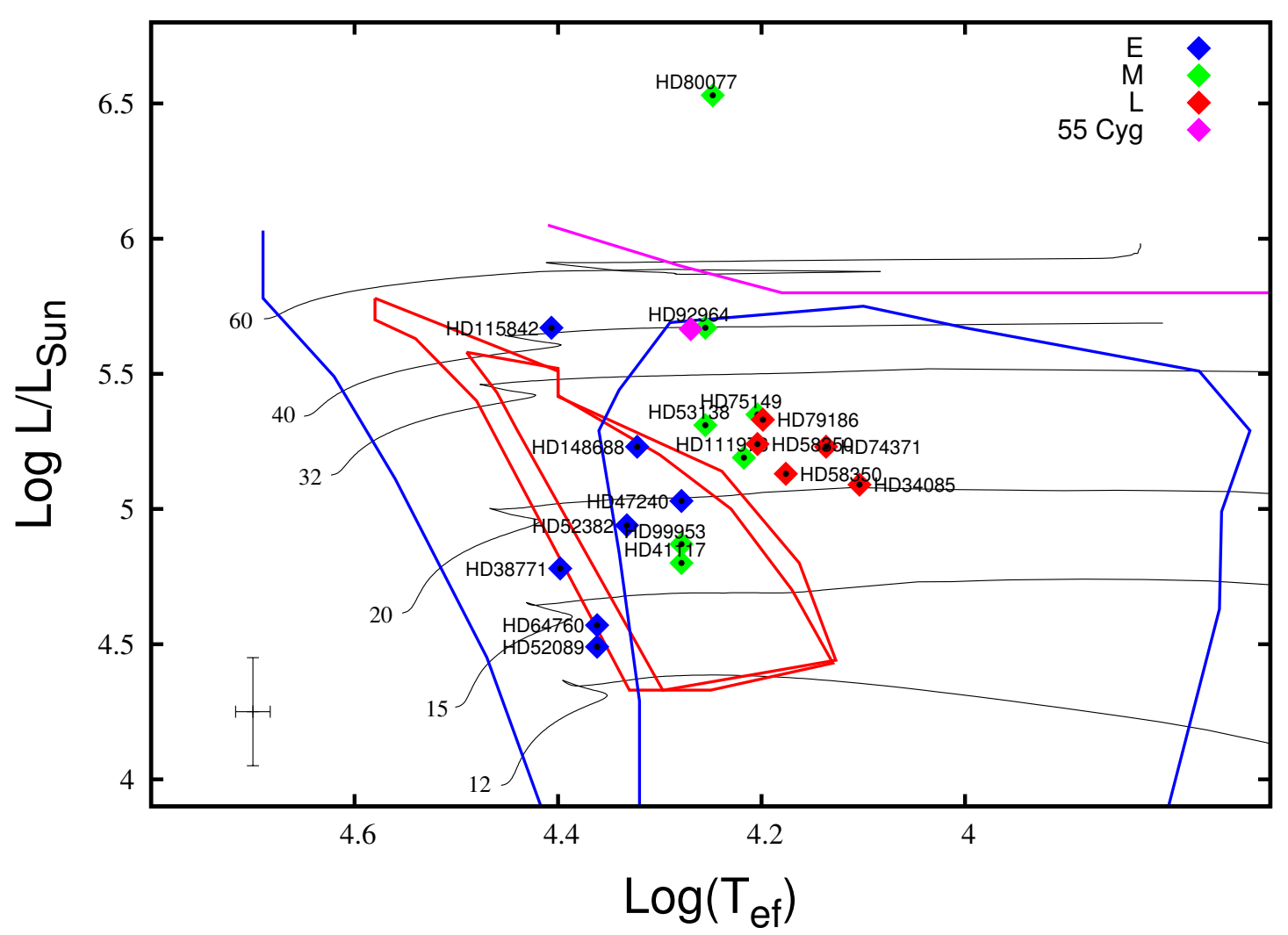

Figura 5.7. Posición de la muestra de estrellas en el diagrama HR. Los caminos evolutivos (tomados de Ekström et al. 2012, líneas negras) corresponden a modelos sin rotación de estrellas con masas iniciales de $\mathrm{M}_{\star}=12,15,20,32,40$ y $60 \mathrm{M}_{\odot}$. Las curvas de colores definen las regiones de inestabilidad de varios modos de pulsación (Saio 2011): a) para modos $\mathrm{p}$ radiales y no radiales de bajo orden (líneas azules); b) modos g no radiales con $l=1$ y 2 (líneas rojas); c) modos de oscilación convectivos (líneas celeste); d) modos radiales monótonamente inestables (por arriba de la línea magenta, y donde se encuentra la estrella hipergigante HD 80077. Los diamantes azules, verdes, rojos y magenta corresponden a las BSGs tempranas (E), medias (M), tardías (L) y 55 Cyg, respectivamente. 
dancias de algún elemento, principalmente de $\mathrm{C}$, no podemos concluir si son objetos que ya han pasado por la etapa de RSG o no. Sin embargo, cabe mencionar que las imágenes de WISE revelan signos de interacciones previas entre el viento de las estrellas HD 52382, HD 99953 y HD 115842 y el medio circunestelar (ver cap. 3). En principio estas tres estrellas tienen distintas clasificaciones (ver tabla 5.1), con lo cual, no necesariamente las imágenes de WISE revelarían que estas estrellas podrían estar en una etapa de post-RSG. Recordemos del cap. 1. fig. 1.1, que si la estrella es lo suficientemente masiva, sus vientos en la secuencia principal pueden ser lo suficientemente fuertes como para desprender sus capas más externas.

En la fig. 5.7 se resalta la estrella 55 Cyg en magenta dado que esta estrella ha sido analizada desde otro punto de vista. De acuerdo con la temperatura efectiva y luminosidad determinada (ver tabla 4.1), observamos que estos valores se corresponden con la evolución de una estrella con un camino evolutivo de $40 \mathrm{M}_{\odot}$, sin embargo determinar el estado evolutivo ubicando simplemente a la estrella en el diagrama HR no es suficiente, ya que podría estar por primera o segunda vez en la región de las supergigantes B (Saio et al. 2013b. Un criterio desarrollado por Saio et al. 2013a, propone que durante la etapa de post-supergigante roja (RSG), las BSGs pueden pulsar en muchos modos, y en particular, en modos radiales extraños, lo cual parece ser imposible si la estrella está evolucionando hacia la etapa de RSG. Más aún, las observaciones de las propiedades pulsacionales con muchos modos excitados son posibles solamente si el objeto experimentó una fuerte pérdida de masa durante la fase de RSG.

Las numerosos períodos identificados espectroscópicamente en 55 Cyg (ver apéndice 6. son una mezcla de modos p y g. De acuerdo con los cálculos de pulsaciones en BSGs, para modelos con rotación y masas iniciales entre $20 \mathrm{M}_{\odot}$ y $25 \mathrm{M}_{\odot}$, la coexistencia de los dos tipos de pulsación en estadíos de pre-RSG se encuentra sólo para estrellas con temperaturas $\geq 20000 \mathrm{~K}$, mientras que en las más frías se espera encontrar muy pocas pulsaciones Saio et al. 2013a. Como la temperatura de 55 Cyg ronda los $19000 \mathrm{~K}, \mathrm{y}$ si bien la masa estelar parece ser mayor a las masas de los modelos mencionados, estas periodicidades son muy numerosas como para que la estrella se encuentre en una etapa de pre-RSG. Consecuentemente, el escenario de post-RSG es el más plausible.

En apoyo a este escenario, se observa la presencia de una estructura de arco de choque (ver imagen en la banda W4 $(24 \mu \mathrm{m})$ del satélite infrarrojo WISE (Wright et al. 2010, fig. 5.8), que parece haber sido formada en el pasado debido a la interacción de una fase de fuertes vientos estelares con el medio interestelar.

Sin embargo, esta etapa evolutiva (post-RGB), requiere de abundancias superficiales de He mayores (casi el doble) que para la etapa pre-RGB (Saio et al. 2013b), situación que contradice nuestras observaciones dado que nuestros modelos indican que la abundancia de He de 55 Cyg es similar a la solar. Por otro lado, esta contradicción también fue observada en Rigel y Deneb Saio et al. 2013b. Para resolver esta discrepancia, Georgy et al. 2014) mostraron que los nuevos cálculos de evolución estelar, basados en el criterio de Ledoux 3

\footnotetext{
${ }^{3}$ En esencia este criterio es igual al criterio de Schwarzschild, que analiza los desplazamientos adiabáticos de las burbujas convectivas respecto a su posición de equilibrio. Pero el criterio de Ledoux también habilita
} 


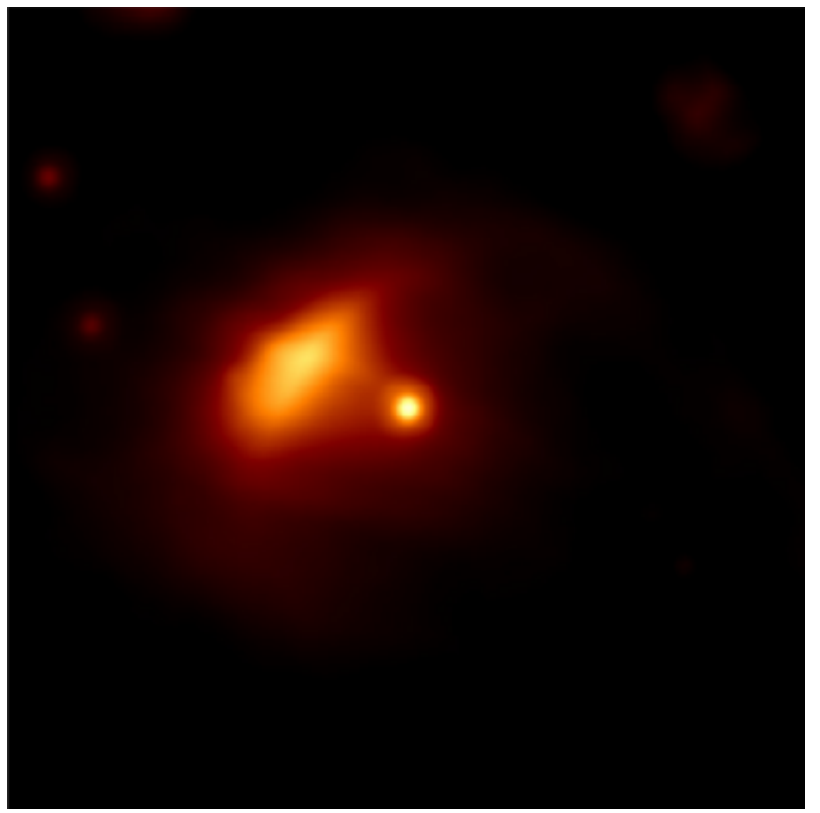

Figura 5.8. Imagen del satélite WISE en la banda W4 $(24 \mu \mathrm{m})$ de 55 Cyg. Se observa una clara estructura de arco de choque. El tamaño de la imagen es de 10'x10'.

para la convección, reconcilian las propiedades pulsacionales esperadas en la fase de postRSG con abundancias químicas superficiales de He bajas.

\subsection{Relación momento-luminosidad del viento (WLR)}

Una manera muy sencilla de comparar las propiedades del viento de las estrellas masivas es analizando la relación momento-luminosidad del viento (WLR). La WLR empírica para las estrellas masivas está en general representada linealmente mediante la ecuación 2.29 (ver cap. 2):

$$
\log D_{\mathrm{mom}}=X \log L / L_{\odot}+D_{0}
$$

Valores para los coeficientes $\mathrm{X}=1 / \alpha_{\text {ef }}$ y $\mathrm{D}_{0}$ reportados en la literatura por diferentes autores están detallados en la tabla 5.2 en conjunto con el parámetro $\alpha_{\text {ef }}$ y los errores correspondientes. Resultados previos indican que cuando las tasas de momento del viento son derivadas de modelos con blanketing, los coeficientes derivados de la WLR son sistemáticamente mayores a aquellos valores encontrados por Kudritzki et al. 1999. Por otra parte, los coeficientes $\left(\mathrm{X}, \mathrm{D}_{0}\right)$ calculados en este trabajo para las estrellas tempranas $(\mathrm{E})$, son cercanos a los obtenidos por Herrero et al. 2002 y Mokiem et al. 2007, quienes usaron una muestra de estrellas que en su mayoría consistía en estrellas OSG. Ambos coeficientes

la posibilidad de que si existe un gradiente de abundancias químicas, la burbuja convectiva tenga una composicion química diferente que la del entorno. 
Tabla 5.2. Coeficientes de la relación momento-luminosidad derivada por diferentes autores.

\begin{tabular}{|c|c|c|c|c|}
\hline Referencia & $\mathrm{X}$ & $\overline{\mathrm{D}_{0}}$ & $\alpha_{\mathrm{ef}}$ & Muestra usada \\
\hline Kudritzki et al. 1999 & $1,34 \pm 0,25$ & $21,24 \pm 1,38$ & & $\mathrm{E}$ \\
\hline Kudritzki et al. 1999 & $1,95 \pm 0,2$ & $17,07 \pm 1,05$ & & M \\
\hline Vink 2000 & $1,826 \pm 0,044$ & $18,68 \pm 0,26$ & & $\mathrm{~T}_{\text {ef }}>27,5 \mathrm{kK}$ de teoría \\
\hline Vink $\sqrt{2000}$ & $1,914 \pm 0,043$ & $18,52 \pm 0,23$ & & $12.5 \mathrm{kK}<\mathrm{T}_{\mathrm{ef}}<22,5 \mathrm{kK}$ de teoría \\
\hline Herrero et al. 2002 & $2,18 \pm 0,21$ & $16,81 \pm 1,16$ & $0,46 \pm$ & mayormente OSGs \\
\hline Mokiem et al.] 2007 & $1,84 \pm 0,17$ & $18,87 \pm 0,98$ & $0,54 \pm 0,05$ & mayormente OSGs \\
\hline Searle et al. 2000 & $1,59 \pm \mathrm{n} / \mathrm{m}$ & $19,86 \pm 0,78$ & $0,63 \pm 0,06$ & $\mathrm{E} \& \mathrm{M}$ \\
\hline Este trabajo & $1,96 \pm 0,28$ & $17,98 \pm 1,43$ & $0,51 \pm 0,07$ & $\mathrm{E}$ \\
\hline Este trabajo & $1,43 \pm 0,42$ & $19,94 \pm 2,23$ & $0,70 \pm 0,21$ & M \& L \\
\hline
\end{tabular}

$\mathrm{E}, \mathrm{M}, \mathrm{y} \mathrm{L}$ se refieren a tipos espectrales B tempranos, medios y tardíos, respectivamente. Searle et al. 2008a no hacen mención del error en el parámtro X

también concuerdan con las predicciones teóricas de Vink 2000 para las estrellas con $\mathrm{T}_{\text {ef }}$ $>27000 \mathrm{~K}$, pero discrepan con los valores obtenidos por Searle et al. 2008a. Todas estas relaciones están representadas en la fig. 5.9.

En particular encontramos que la diferencia entre nuestros valores $\left(\mathrm{X}, \mathrm{D}_{0}\right)$ y los de Searle et al. 2008a podría deberse al hecho de que la muestra de estrellas seleccionada por estos autores es una mezcla de estrellas BSGs de tipos espectrales tempranos y medios. De hecho, si separamos la muestra de Searle et al. en dos subgrupos, obtenemos $\mathrm{X}=1.85$ $\pm 0.36 \mathrm{y}_{0}=18.47 \pm 2.00$ para las BSGs tempranas, y $\mathrm{X}=1.35 \pm 0.14$ y $\mathrm{D}_{0}=21.08$ \pm 0.73 para las BSGs de tipos espectrales medios, coincidiendo así los coeficientes con nuestros valores.

Algo interesante a remarcar es que las observaciones caen sistemáticamente por debajo de la relación teórica y Vink 2000 atribuye este resultado a errores sistemáticos en los valores observados, mientras que Markova \& Puls 2008) sugieren que la presencia de clumping podría incluso hacer más marcada la diferencia ya que sólo afectaría marginalmente la predicción pero los valores observados de $\dot{M}$ estarían sobreestimados.

\subsection{Salto de biestabilidad}

Como vimos en el cap. 2, secc. 2.9.1, existe una región de biestabilidad alrededor del tipo espectral B1 $\left(\mathrm{T}_{\text {ef }} \sim 21000 \mathrm{~K}\right)$ donde se observan cambios muy significativos del cociente $\mathrm{v}_{\infty} / \mathrm{v}_{\mathrm{esc}}$.

Con respecto a nuestras observaciones es interesante remarcar que encontramos 6 estrellas en la región de biestabilidad. Particularmente HD 99953 y 55 Cyg muestran pronunciadas variaciones de $\mathrm{H}_{\alpha}$ de la cual diferentes estimaciones de $\dot{\mathrm{M}} \mathrm{y} \mathrm{v}_{\infty}$ fueron realizadas. En el caso de HD 99953 obtuvimos $250 \mathrm{~km} / \mathrm{s}, 500 \mathrm{~km} / \mathrm{s}$ y $700 \mathrm{~km} / \mathrm{s}$, y $\dot{M} 0.08 \times 10^{-6} \mathrm{M}_{\odot} /$ año, $0.13 \times 10^{-6} \mathrm{M}_{\odot} /$ año, y $0.22 \times 10^{-6} \mathrm{M}_{\odot} /$ año. Esto implica un crecimiento tanto en $\mathrm{v}_{\infty}$ como en $\dot{M}$ en un factor $\sim 2.8$. Este resultado contradice la predicción teórica hecha por Vink et al. 1999 quienes argumentan que si el momento del viento $\dot{\mathrm{M}} \mathrm{v}_{\infty}$ fuera constante en el 


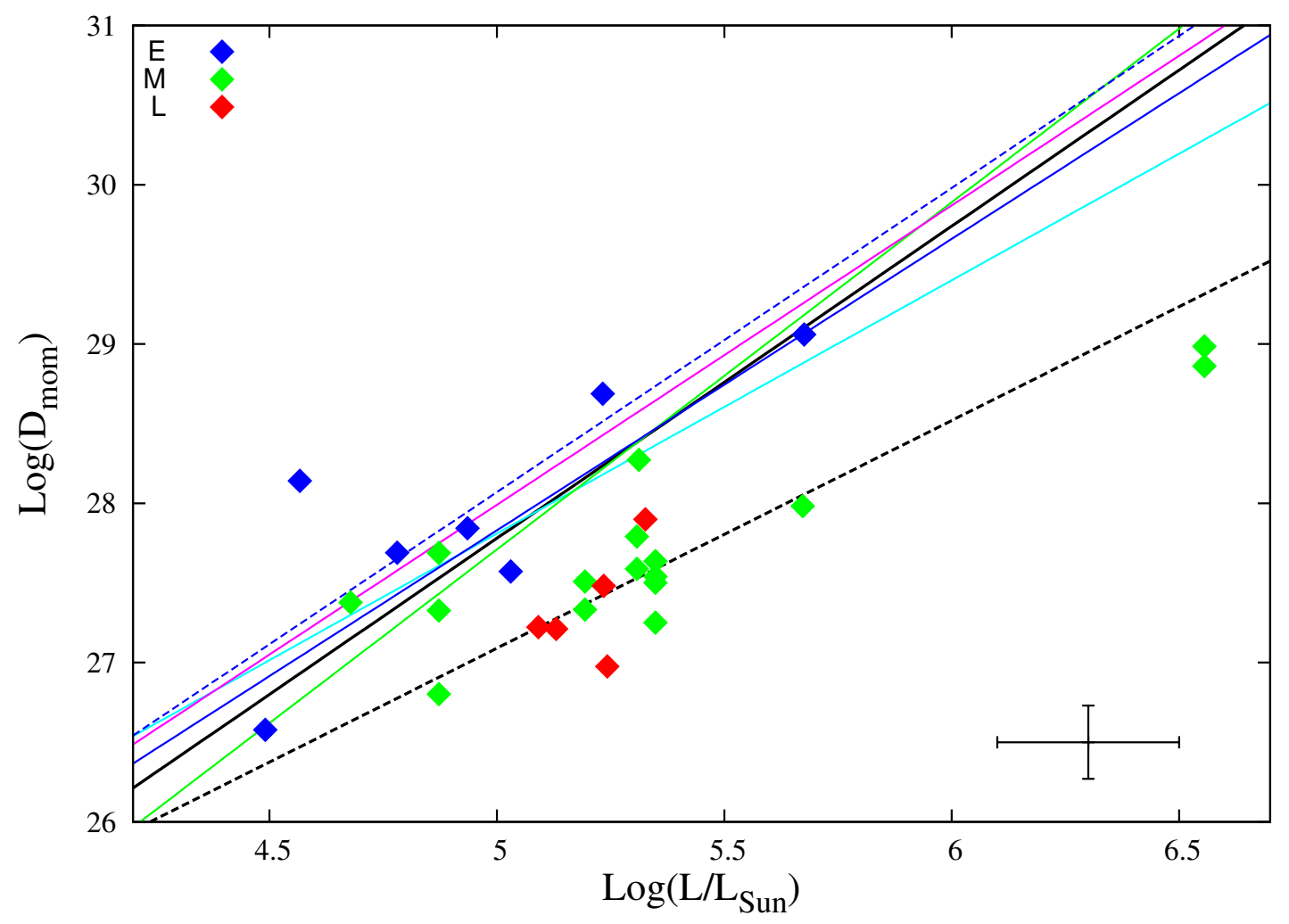

Figura 5.9. Comparación de varias relaciones momento-luminosidad del viento empíricas: este trabajo (líneas sólida y a rayas negras para las estrellas BSGs de tipos espectrales tempranos y medios/tardíos, respectivamente), Herrero et al. (2002 línea sólida verde), Searle et al. 2008a línea sólida celeste), y Mokiem et al. (2007 línea sólida magenta). Las WLR teóricas de Vink 2000 están caracterizadas por líneas azules sólida y a rayas para $T_{\text {ef }}>27500 \mathrm{~K}$ y $T_{\text {ef }}<22500 \mathrm{~K}$, respectivamente. La figura incluye la muestra de estrellas mostrada en la fig. 3.9 
salto de biestabilidad, la tasa de pérdida de masa se incrementaría marcadamente en un factor dos en estrellas de tipos espectrales más tempranos que B1 a más tardíos que B1, y el cociente $\mathrm{v}_{\infty} / \mathrm{v}_{\text {esc }}$ decrecería de 2.6 a 1.3 .

Si comparamos ahora los parámetros del viento medidos para HD 99953 con los resultados teóricos encontrados por Vink et al. 1999 para $\mathrm{T}_{\text {ef }}=20000 \mathrm{~K}, \mathrm{v}_{\infty} / \mathrm{v}_{\text {esc }}=2.6, \mathrm{R}_{\star}$ $=26.4 \mathrm{R}_{\odot} \mathrm{y} \log \dot{\mathrm{M}}=-6.54$ (ver tabla 5.10 marcado en celeste), concuerdan muy bien con nuestras determinaciones de $R_{\star}\left(25 R_{\odot}\right)$ y $\log \dot{M}$ (-6.66). Sin embargo, el valor que ellos proponen para la $\mathrm{v}_{\infty}(1300 \mathrm{~km} / \mathrm{s})$ es el doble del que nosotros estimamos para este objeto $(700 \mathrm{~km} / \mathrm{s})$.

Encontramos que no podemos atribuir esta discrepancia a la luminosidad de la estrella, ya que la que calculamos $\left(\log \mathrm{L} / \mathrm{L}_{\odot}=4.87 \pm 0.37\right)$ coincide con el valor teórico propuesto de $\log \mathrm{L} / \mathrm{L}_{\odot}=5$ y tampoco encontramos que la diferencia hallada esté relacionada con la ley de velocidad adoptada para el modelo.

Si consideramos otras estrellas con $\log \mathrm{L} / \mathrm{L}_{\odot} \sim 5$ (ver tabla 6.1), encontramos que los parámetros del viento de las estrellas ubicadas del lado caliente de la región de biestabilidad, como ser HD $38771\left(\mathrm{~T}_{\text {ef }}=25000 \mathrm{~K}, \log \dot{\mathrm{M}}=-6.85 \mathrm{y} \mathrm{v}_{\infty}=1500 \mathrm{~km} / \mathrm{s}\right)$ y HD $52382\left(\mathrm{~T}_{\mathrm{ef}}=\right.$ $21500 \mathrm{~K}, \log \dot{\mathrm{M}}=-6.62 \mathrm{y} \mathrm{v}_{\infty}=1000 \mathrm{~km} / \mathrm{s}$ ) se parecen a aquellos valores teóricos listados en la tabla de la fig. 5.10. En contraste, las estrellas ubicadas del otro lado de la región de biestabilidad, como ser HD $34085\left(\mathrm{~T}_{\text {ef }}=12700 \mathrm{~K}, \log \dot{\mathrm{M}}=-6.64 \mathrm{y} \mathrm{v} \infty / \mathrm{v}_{\text {esc }}=0.8\right)$, HD $58350\left(\mathrm{~T}_{\mathrm{ef}}=15000-16000 \mathrm{~K} \log \dot{\mathrm{M}}=-6.82 \mathrm{y} \mathrm{v}_{\infty} / \mathrm{v}_{\mathrm{esc}}=0.9\right)$, y HD $111973\left(\mathrm{~T}_{\mathrm{ef}}=\right.$ $16500 \mathrm{~K}, \log \dot{\mathrm{M}}$ entre $\left.-6.7 \mathrm{y}-6.85, \mathrm{y} \mathrm{v}_{\infty} / \mathrm{v}_{\mathrm{esc}}=1.4\right)$ tienen velocidades terminales y tasas de pérdida de masa menores que las esperadas de los modelos calculados por Vink et al. 1999. Esto significa que un decrecimiento en el cociente $\mathrm{v}_{\infty} / \mathrm{v}_{\text {esc }}$, tanto dentro como del lado frío del salto de biestabilidad, no es acompañado necesariamente por un incremento en la tasa de pérdida de masa. Este resultado apoya las conclusiones de Markova \& Puls 2008 quienes encontraron también que tanto $\dot{M}$ como $v_{\infty}$ decrecen en el mismo sentido.

Interpretamos que $\dot{\mathrm{M}} \mathrm{v}_{\infty}$ no es constante en el salto de biestabilidad y que el decrecimiento en $\dot{\mathrm{M}}$ y $\mathrm{v}_{\infty}$ en el lado frío del salto podría explicarse con la solución hidrodinámica $\delta$-lenta para vientos impulsados por radiación encontrada por Curé et al. 2011). Estos autores reportaron que un cambio en la ionización del viento, caracterizado por el parámetro $\delta$ de la fuerza de la línea, define dos regímenes diferentes de vientos estacionarios (rápido y lento). Por otra parte, Venero et al. 2016) demuestran que el régimen rápido está siempre presente a altas temperaturas $(>25000 \mathrm{~K})$ mientras que en estrellas BSGs de tipo espectral medio podrían desarrollarse vientos rápidos o vientos lentos. Los dos regímenes están separados por una zona de inestabilidad donde existe un flujo no estacionario. Estos autores demuestran además que los modelos calculados con la solución $\delta$-lenta a temperaturas $<17000 \mathrm{~K}$, predicen un decrecimiento tanto en la velocidad terminal como en la tasa de pérdida de masa como puede observarse en la fig. 5.11. en coincidencia con nuestros resultados.

Venero et al. 2016 proponen que una perturbación en la densidad de la base del viento podría desencadenar un cambio de un régimen lento a uno rápido, y viceversa. Este comportamiento podría explicar la alta variabilidad del viento que encontramos en HD 99953 y 55 Cyg, que son dos estrellas que se ubican en la región de biestabilidad. 


\begin{tabular}{|c|c|c|c|c|c|c|c|c|}
\hline $\mathrm{v}_{\infty} / \mathrm{v}_{\infty \infty}$ & $\begin{array}{l}T_{\text {eff }} \\
(\mathrm{K})\end{array}$ & $\begin{array}{r}R_{*} \\
\left(R_{\odot}\right)\end{array}$ & $\begin{array}{r}v_{\text {eac }} \\
\left(\mathrm{km} \mathrm{s}^{-1}\right)\end{array}$ & $\left(\begin{array}{r}v_{\infty} \\
\left(\mathrm{km} \mathrm{s}^{-1}\right)\end{array}\right.$ & $\begin{array}{c}\log \dot{M} \\
\left(M_{\odot} / y r\right)\end{array}$ & $\eta$ & $\begin{array}{c}\Delta L / L \\
\text { (in } 10^{-3} \text { ) }\end{array}$ & model \\
\hline \multirow{12}{*}{1.3} & 12500 & 67.7 & 310 & 410 & -6.32 & 0.095 & 0.103 & \\
\hline & 15000 & 47.0 & 380 & 490 & -6.39 & 0.097 & 0.126 & \\
\hline & 17500 & 34.5 & 440 & 570 & -6.28 & 0.146 & 0.221 & \\
\hline & 20000 & 26.4 & 500 & 650 & -6.22 & 0.192 & 0.332 & \\
\hline & 22500 & 20.9 & 560 & 730 & -6.15 & 0.254 & 0.493 & \\
\hline & 25000 & 16.9 & 630 & 810 & -6.12 & 0.302 & 0.653 & C \\
\hline & 27500 & 14.0 & 690 & 900 & -6.40 & 0.174 & 0.414 & \\
\hline & 30000 & 11.8 & 750 & 980 & -6.58 & 0.126 & 0.326 & \\
\hline & 32500 & 10.0 & 810 & 1060 & -6.58 & 0.136 & 0.382 & \\
\hline & 35000 & 8.6 & 880 & 1140 & -6.43 & 0.207 & 0.626 & \\
\hline & 37500 & 7.6 & 940 & 1220 & -6.37 & 0.255 & 0.826 & \\
\hline & 40000 & 6.6 & 1000 & 1300 & -6.26 & 0.350 & 1.210 & \\
\hline \multirow{12}{*}{2.0} & 12500 & 67.7 & 310 & 630 & -6.74 & 0.056 & 0.073 & \\
\hline & 15000 & 47.0 & 380 & 750 & -6.62 & 0.088 & 0.138 & \\
\hline & 17500 & 34.5 & 440 & 880 & -6.49 & 0.139 & 0.254 & \\
\hline & 20000 & 26.4 & 500 & 1000 & -6.41 & 0.191 & 0.398 & \\
\hline & 22500 & 20.9 & 560 & 1130 & -6.32 & 0.264 & 0.620 & \\
\hline & 25000 & 16.9 & 630 & 1250 & -6.48 & 0.203 & 0.530 & \\
\hline & 27500 & 14.0 & 690 & 1380 & -6.73 & 0.125 & 0.360 & \\
\hline & 30000 & 11.8 & 750 & 1500 & -6.76 & 0.128 & 0.400 & \\
\hline & 32500 & 10.0 & 810 & 1630 & -6.71 & 0.155 & 0.527 & \\
\hline & 35000 & 8.6 & 880 & 1750 & -6.59 & 0.220 & 0.801 & \\
\hline & 37500 & 7.6 & 940 & 1880 & -6.57 & 0.247 & 0.969 & \\
\hline & 40000 & 6.6 & 1000 & 2000 & -6.48 & 0.325 & 1.356 & \\
\hline \multirow[t]{12}{*}{2.6} & 12500 & 67.7 & 310 & 810 & -6.95 & 0.045 & 0.070 & \\
\hline & 15000 & 47.0 & 380 & 980 & -6.85 & 0.067 & 0.126 & \\
\hline & 17500 & 34.5 & 440 & 1140 & -6.69 & 0.114 & 0.248 & \\
\hline & 20000 & 26.4 & 500 & 1300 & -6.54 & 0.184 & 0.458 & \\
\hline & 22500 & 20.9 & 560 & 1460 & -6.59 & 0.184 & 0.517 & \\
\hline & 25000 & 16.9 & 630 & 1630 & -6.79 & 0.129 & 0.403 & B \\
\hline & 27500 & 14.0 & 690 & 1790 & -6.95 & 0.098 & 0.337 & A \\
\hline & 30000 & 11.8 & 750 & 1950 & -6.92 & 0.115 & 0.430 & \\
\hline & 32500 & 10.0 & 810 & 2120 & -6.86 & 0.143 & 0.579 & \\
\hline & 35000 & 8.6 & 880 & 2280 & -6.76 & 0.194 & 0.845 & \\
\hline & 37500 & 7.6 & 940 & 2440 & -6.71 & 0.233 & 1.089 & \\
\hline & 40000 & 6.6 & 1000 & 2600 & -6.68 & 0.266 & 1.327 & \\
\hline
\end{tabular}

Figura 5.10. Parámetros estelares y del viento para una grilla de modelos con $\log \mathrm{L} / \mathrm{L} \odot$ $=5, \mathrm{M}_{\odot}=20 \mathrm{M}_{\odot}, \beta=1 \mathrm{y}$ metalicidad solar (ver Vink et al. 1999). En celeste marcamos el modelo que usamos para comparar los resultados obtenidos para HD 99953. Para mayor detalle ver el texto. 

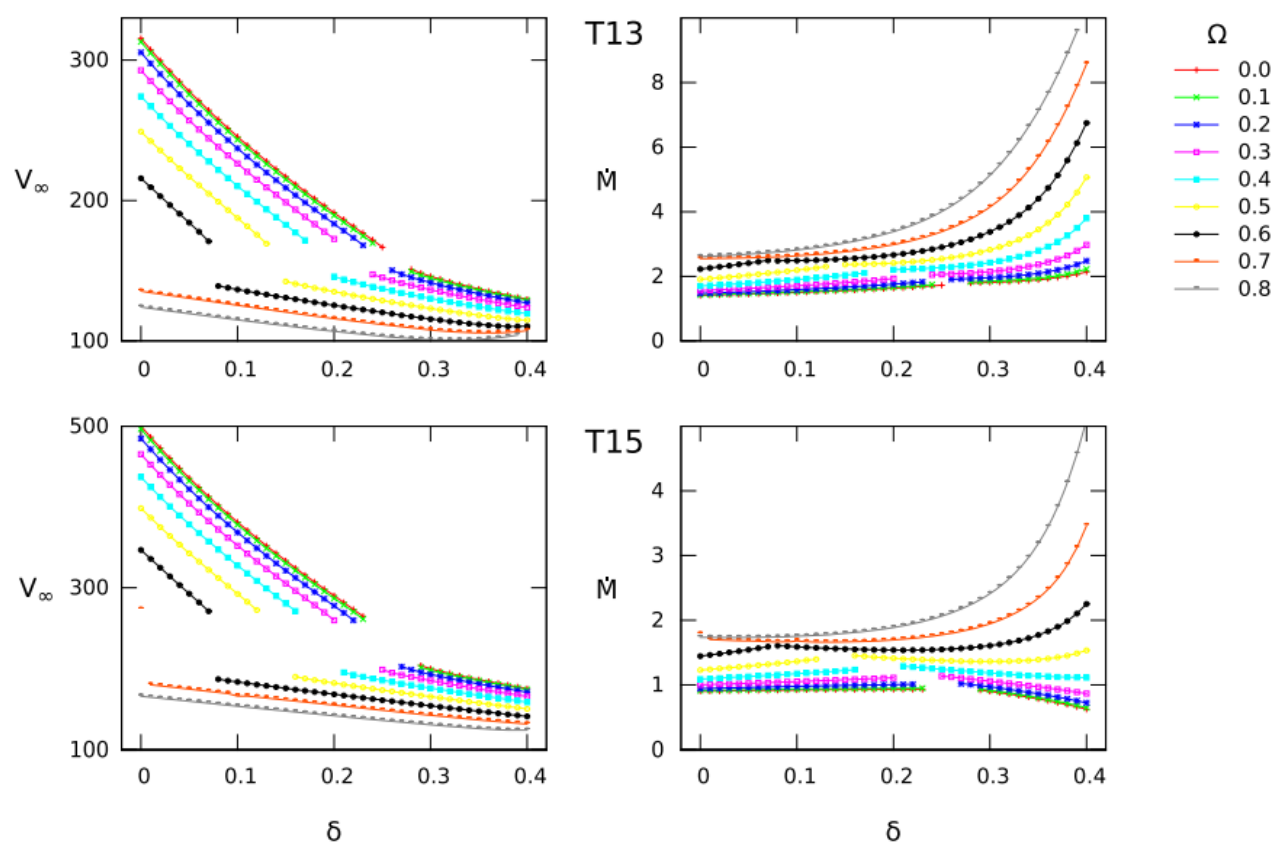

Figura 5.11. Velocidades terminales (paneles izquierdos) y tasas de pérdida de masa (paneles derechos) en función del parámetro $\delta$ de la fuerza de radiación, para diferentes valores de $\Omega$ (cociente entre la velocidad de rotación y la velocidad crítica o de escape) y modelos con $\mathrm{T}_{\text {ef }}$ de $13000 \mathrm{~K}$ (T13) y $15000 \mathrm{~K}$ (T15). Todas las soluciones presentan el quiebre que divide una solución rápida (componentes superiores de $\mathrm{v}_{\infty}$ ) de una solución lenta (componentes inferiores de $\mathrm{v}_{\infty}$ ). Extraído de Venero et al. 2016. 


\subsection{Pulsaciones y pérdida de masa}

Es un hecho bien conocido que las estrellas BSGs muestran variaciones en las regiones espectrales del óptico y del UV que pueden ser atribuidas al movimiento de estructuras de gran escala en el viento Prinja et al. 2002). Los mecanismos sugeridos para explicar estos comportamientos pueden estar asociados con variaciones moduladas por la rotación, como fue reportado, por ejemplo, en HD 14134 (B3Ia) y HD 64760 Morel et al.2004:Prinja et al. 2002, respectivamente) o relacionadados con la presencia de campos magnéticos débiles Henrichs et al. 2003); Morel et al. 2004. Por otro lado, investigaciones más recientes han atribuido que algunas variaciones pueden estar asociadas con las pulsaciones estelares, como fue reportado en HD 50064 (B6Ie) por Aerts et al. 2010a).

La principal evidencia de cambios en la estructura del viento en la región espectral del óptico la brinda la línea $\mathrm{H}_{\alpha}$. Como hemos visto, la mayoría de las estrellas estudiadas en esta tesis muestran variaciones en esta línea tanto en forma como en intensidad, por ejemplo, 55 Cyg, HD 75149, HD 53138 y HD 111973 despliegan episodios donde $\mathrm{H}_{\alpha}$ sufre variaciones dramáticas en la forma del perfil (de absorción pura a $\mathrm{P}$ Cygni y viceversa). Hemos registrado que estas variaciones pueden presentarse en escalas de tiempo que van de horas a días.

Debido a la sensibilidad que existe entre la línea $\mathrm{H}_{\alpha}$ y la tasa de pérdida de masa, resulta importante analizar las variaciones que presenta este parámetro y a través de él poder determinar si existe alguna relación con los fenómenos mencionados al inicio de esta subsección. Teniendo en cuenta la amplitud de variación de la pérdida de masa en objetos individuales, Prinja \& Howarth 1986 midieron un nivel de variabilidad del $10 \%$ en escalas de tiempo de un día o más, mientras que Lefever et al. 2007) han reportado cocientes entre tasas de pérdida de masa máxima y mínima entre 1.05 y 2.5. En este sentido, basándonos en un estudio cuidadoso de la variación de $\mathrm{H}_{\alpha}$ en diferentes épocas, también encontramos variaciones en $\dot{\mathrm{M}}$ pero en un factor 1.5 a 2.7 en 55 Cyg, HD 75149, HD 99953 y HD 111973.

Como la línea $\mathrm{H}_{\alpha}$ es muy sensible a los cambios en la densidad y en los gradientes de velocidad en la base del viento Cidale \& Ringuelet 1993, se espera que inestabilidades fotosféricas produzcan la variabilidad de la línea y cambios en la estructura del viento. Para ahondar en el origen de la variabilidad en los vientos de las estrellas BSGs, recolectamos información de la literatura para todos los objetos estudiados en lo que respecta a períodos en las variaciones de luz y/o espectroscópicos (ver tabla 3.1) como determinaciones previas de los parámetros del viento, ver tabla 6.1. En dicha tabla pueden apreciarse diferencias entre el cociente del máximo y mínimo valor de $\dot{M}$ que van de 1 a 7 . Estas discrepancias no son completamente reales debido a que los modelos fueron computados utilizando diferentes radios estelares, por lo que las diferencias entre los valores de $\dot{M}$ podrían ser menores. Por ejemplo, para HD 34085 se puede observar que hay una discrepancia en $\dot{M}$ de 1.5 si comparamos nuestros resultados con los de Markova \& Puls 2008), pero ésta podría ser 1.3 veces mayor al de esos autores $\left(\dot{M}=0.44 \times 10^{-6} M_{\odot} /\right.$ año $)$ si hubiésemos adoptado un valor de $R_{\star}=115 R_{\odot}$ (manteniendo constante el resto de los parámetros).

Por lo tanto, para comparar los valores obtenidos en este trabajo con los calculados por diferentes autores deberíamos discutir las propiedades de escala de los diferentes modelos 
ya que, como mencionamos en el cap. 2, secc. 2.9 los modelos no son únicos (Puls et al. 2008 , debido a que para diferentes combinaciones de $\dot{\mathrm{M}}, \mathrm{v}_{\infty} \mathrm{y} \mathrm{R}_{\odot}$ se pueden obtener los mismos perfiles de línea. Por este motivo adoptamos el invariante de profundidad óptica $\mathrm{Q}_{\mathrm{r}}$ $=\dot{\mathrm{M}} / \mathrm{R}_{\star}^{1,5}$, en lugar del parámetro tradicional $\mathrm{Q}=\dot{\mathrm{M}} /\left(\mathrm{v}_{\infty} \mathrm{R}_{\star}\right)^{1,5}$ Puls et al. 1996), ya que consideramos que $\mathrm{v}_{\infty}$ puede cambiar debido a la variabilidad del viento, como observamos en 55 Cyg. Para poder comparar las observaciones, en la tabla 6.1 mostramos los cálculos de $Q_{r}$, obtenidos a partir de los resultados de diferentes autores con sus correspondientes errores. Estos cálculos muestran que la diferencia en $\log Q_{r}$ es 2 o 3 veces mayor que sus correspondientes barras de error, sugiriendo variaciones reales en los parámetros del viento, dado que las observaciones corresponden a diferentes épocas.

Luego, comparamos la amplitud de variación de la tasa de pérdida de masa (es decir el cociente entre los valores máximo y mínimos medidos), usando el parámetro $\mathrm{Q}_{\mathrm{r}}$ con los máximos períodos listados en la tabla 3.1 del capítulo 3.

Como se muestra en la fig. 5.12, encontramos una relación lineal para la mayoría de las estrellas BSGs de tipos espectrales medios y tardíos (B2 - B9) con P > 6 días. Agregamos a esta relación los datos de 55 Cyg y Deneb (cuyos datos fueron extraídos de los trabajos de Chesneau et al. 2010, Scuderi et al. 1992. Observamos que cuanto mayor es el período, menor es la amplitud del parámetro $\mathrm{Q}_{\mathrm{r}}$. En la fig. 5.12 puede observarse que tres BSGs de mediano tipo espectral con valores medidos de $\mathrm{P}$ y $\mathrm{M}$ no fueron tenidas en cuenta en la relación: HD 53138, HD 75149 y HD 99953 (puntos verdes sin triángulo). Las estrellas HD 75149 y HD 99953 no han sido muy estudiadas y probablemente nuevos períodos deberían ser determinados. Particularmente la primera tiene valores poco precisos de $\dot{M}$ ya que dos de nuestros perfiles están en absorción pura. La estrella HD 111973 no fue incluida debido a que sólo tenemos observaciones correspondientes a dos días consecutivos, intervalo de tiempo que es menor que el mayor período de variación reportado (57.11 días).

La relación que obtuvimos es $\mathrm{P}_{\max }=100.27( \pm 7.64) \mathrm{X}+83.97( \pm 4.47)$, donde $\mathrm{X}=$ $\log \mathrm{Q}_{\mathrm{r}_{\min }} / \mathrm{Q}_{\mathrm{r}_{\max }} \mathrm{y}$ fue determinada usando los datos referenciados con un triángulo en la figura 5.12 (los valores usados de $\mathrm{Q}_{r_{\min }}$ y $\mathrm{Q}_{r_{\max }}$ están listados y remarcados en la tabla 6.1. Las diferencias en los valores seleccionados son dos o tres veces mayores que sus correspondientes errores, y sugiere que las variaciones en la pérdida de masa son reales.

En cambio, los valores de la amplitud de variación del parámetro $\mathrm{Q}_{\mathrm{r}}$ correspondiente a las estrellas BSGs de tipo espectral temprano (HD 38771, HD 47240, HD 52089, HD 64740 y HD 148688, indicadas con diamantes azules) no muestran una correlación clara con sus períodos. Pero éstas presentan diferencias en sus parámetros $Q_{\mathrm{r}}$ del orden del error de dicho parámetro, con lo cual no podemos cuantificar la variación de sus vientos. 


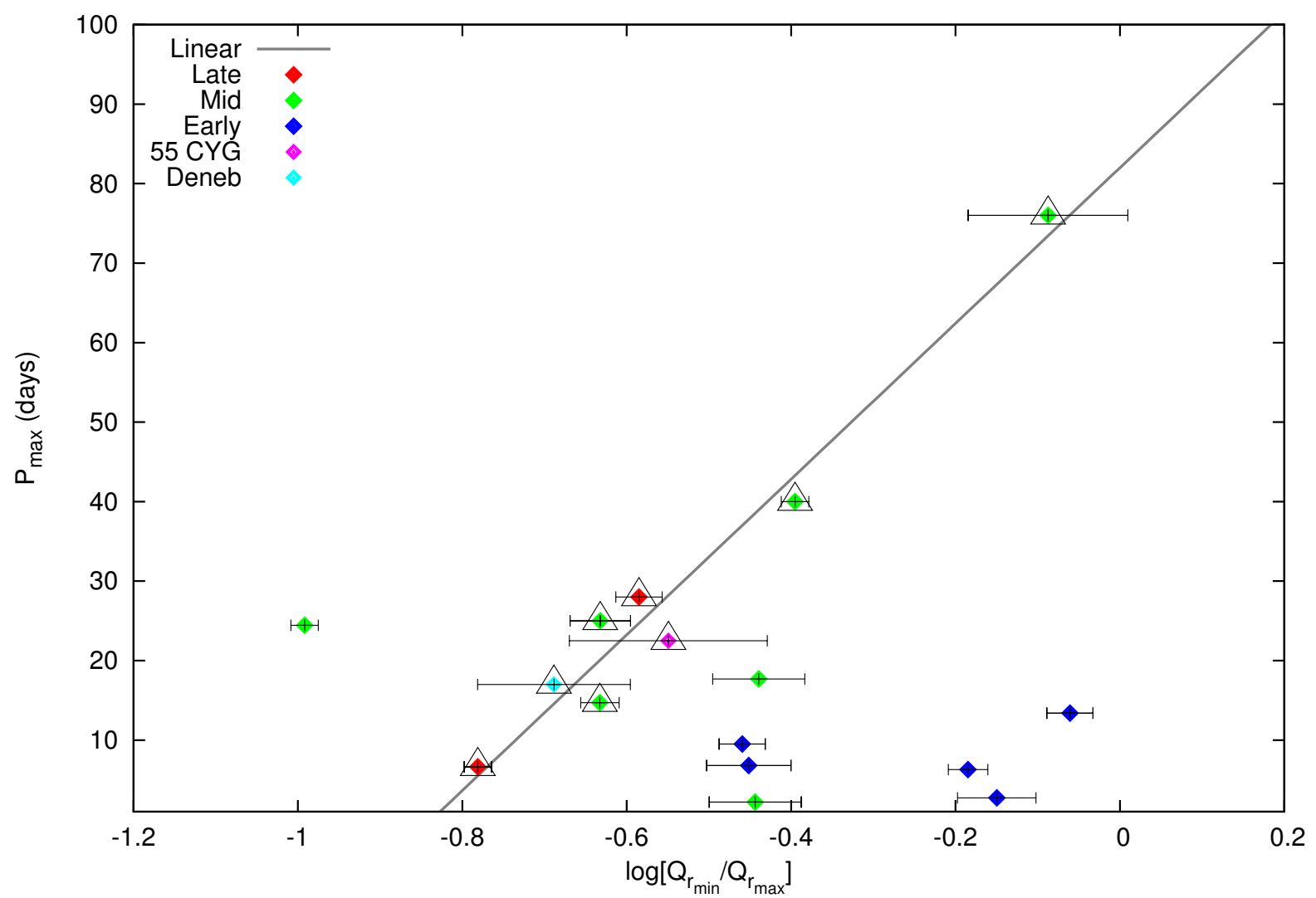

Figura 5.12. Cociente entre los valores mínimo y máximo de $\mathrm{Q}_{\mathrm{r}}=\dot{M} / R_{\star}^{1,5}$ como función del período fotométrico/espectroscópico más largo reportado en la literatura. La relación lineal fue obtenida usando los datos indicados con un triángulo. 



\section{Capítulo 6}

\section{Conclusiones}

En este trabajo hemos estudiado una muestra de 20 estrellas B supergigantes galácticas mediante el ajuste de líneas espectrales de H, He, y Si con el código FASTWIND.

A partir del análisis de síntesis espectral pudimos determinar los parámetros estelares y del viento de estas estrellas. Teniendo en cuenta los errores involucrados en la temperatura efectiva $( \pm 1000 \mathrm{~K})$ y en la gravedad superficial $( \pm 0.1$ dex$)$, la mayoría de nuestros resultados están de acuerdo con los valores obtenidos previamente por otros autores. Sin embargo, hemos encontrado diferencias significativas en la determinación de los radios estelares. Puesto que los valores que determinamos son valores medios que se obtuvieron mediante métodos independientes, consideramos que las medidas reportadas en este trabajo son precisas.

En lo que respecta a los parámetros del viento hemos obtenido nuevas determinaciones de tasas de pérdida de masa y velocidades terminales. Hemos reportado para tres estrellas (HD 74371, HD 99953 y HD 111973) los parámetros del viento por primera vez. En relación a las propiedades globales de los vientos, encontramos dos comportamientos diferentes en la relación momento-luminosidad: una de estas relaciones se corresponde a las estrellas de tipos espectrales tempranos y medios que siguen una ley de velocidad con valores de $\beta \leq$ 2 y poseen velocidades terminales mayores a $500 \mathrm{~km} / \mathrm{s}$. Mientras que el otro grupo está caracterizado por las estrellas de tipos espectrales medios y tardíos con $\beta \geq 2$ y velocidades terminales menores a $500 \mathrm{~km} / \mathrm{s}$. La relación observada para el subgrupo de estrellas más calientes está de acuerdo con las predicciones teóricas de la teoría estándar y la relación observada para las estrellas de menor temperatura cae sistemáticamente por debajo de la relación teórica. Para este último caso proponemos que los vientos de estas estrellas podrían explicarse con la solución $\delta$-lenta, es decir, que estos vientos presentarían cambios en la estructura de ionización.

Por otra parte, hemos observado que muchas estrellas BSGs de nuestra muestra presentan variaciones en la línea $\mathrm{H}_{\alpha}$, indicando importantes cambios en la estructura del viento. En particular, 55 Cyg, HD 75149, HD 99953 y HD 111973 muestran episodios donde $\mathrm{H}_{\alpha}$ sufre variaciones dramáticas que hemos interpretado como variaciones en la tasa de pérdida de masa en un factor de 1.5 a 2.7 .

En el caso particular de 55 Cyg, para la cual disponíamos de gran cantidad de observa- 
ciones, pudimos encontrar 19 frecuencias de pulsación. La presencia de múltiples períodos, la masa derivada espectroscópicamente, $\mathrm{M}_{\star}=32 \mathrm{M}_{\odot}$, y la evidencia de grandes eyecciones de masa en el pasado, nos lleva a pensar que 55 Cyg está probablemente en un fase evolutiva de post-RSG. Así, la estrella posee las características propias de una variable $\alpha$ Cygni.

En lo que respecta a la evolución de las estrellas BSGs de la muestra, hemos encontrado un grupo estrellas de tipo espectral temprano que habrían terminado recientemente la secuencia principal, y otro grupo de estrellas que se encuentran más evolucionadas. Según las regiones de inestabilidad del diagrama HR concluimos que el primer grupo se encuentra en la región donde los modos radiales están excitados, mientras que el otro se ubica en regiones donde los modos radiales y no-radiales, e incluso los modos extraños, están excitados. Esta diferencia evolutiva también se ve reflejada en los valores medidos para los anchos equivalentes de CNO. Por ejemplo, composiciones normales de CNO pueden observarse en las estrellas de post-secuencia o próxima a ella, mientras que las más evolucionadas presentarían sobreabundancias en $\mathrm{N}$, o en $\mathrm{C}$, o claras evidencias de material moderadamente procesado. Esta primera determinación de anchos equivalentes nos ha permitido detectar que los mayores anchos equivalente medidos en $\mathrm{N}$ se corresponden con supergigantes de tipos espectrales entre B1 y B2, y los valores máximos para el C los encontramos para los tipos espectrales entre B3 y B6. Sin embargo, no encontramos anomalías en el He superficial, siendo la abundancias de este elemento similar a la solar.

Las imágenes infrarrojas de WISE revelan la existencia de estucturas de gran escala en 4 estrellas (HD 52382, HD99953, HD 115842 y 55 Cyg), originadas en fases evolutivas previas, indicando la existencia de fases evolutivas previas con enormes pérdidas de masa.

Un resultado a destacar de esta tesis es que pudimos vincular las variaciones de los vientos de las estrellas supergigantes B de tipos espectrales medios/tardíos con los periodos más largos de variaciones de luz encontrados en la literatura. La relación lineal empírica encontrada, relación período-pérdida de masa, asocia la amplitud de la variación de la pérdida de masa parametrizada a través del parámetro $\mathrm{Q}_{\mathrm{r}}$, con el período mencionado. Esta relación podría indicar que los modos radiales extraños de pulsación con períodos mayores a 6 días sean las responsables de las variaciones del viento. Sin embargo, para confirmar esta hipótesis es necesario realizar más observaciones.

Finalmente, podemos concluir que como los perfiles de las líneas observados pueden ser ajustados usando una ley de velocidades tipo $\beta$, la presión de radiación es el principal mecanismo para impulsar el viento. Sin embargo, los modos de pulsación también podrían jugar un papel significativo a la hora de desprender materia en forma (cuasi) periódica, que luego termina siendo acelerado por la radiación. En este sentido, los modos de pulsación radiales extraños con períodos $>6$ días podrían ser los responsables de la variación del viento en las estrellas BSGs de tipos espectrales B2 a B9.

Como las estrellas BSGs son objetos con pulsaciones estables esto permite estudiarlas desde otra perspectiva. Basados en los métodos establecidos por la astrosismología, es posible investigar no sólo la atmósfera estelar desde donde la pérdida de masa se inicia, sino también analizar el interior de estas estrellas para tratar de revelar importantes propiedades físicas. El conocimiento de estos parámetros es de vital importancia para el entendimiento 
de las fases evolutivas de estrellas masivas de post-secuencia, ya que son parámetros claves en los cálculos más modernos de evolución. Esto enfatiza la necesidad de realizar más investigaciones, tanto teóricas como observacionales. En particular, en lo que respecta a las observaciones, son necesarias campañas de observación extendidas en el tiempo para obtener conjutamente periodos de oscilación y tasa de pérdida de masa. 



\section{Apéndice A}

En la tabla 6.1 se muestran los valores de los parámetros estelares y del viento determinados en el cap. 3 en conjunto con datos de la literatura. Los valores de $Q_{r}$ que están resaltados en negro son lo que se usaron para determinar la relación entre el máximo período observado y la amplitud del parámetro $Q_{r}$.

También se muestran en las figuras 6.1, 6.2, 6.3, 6.4, 6.5, 6.6, y 6.7 los resultados de los mejores ajustes a las líneas fotosféricas. Los diferentes modelos, cuyos parámetros están listados en la tabla 3.3 se encuentran en color azul o verde, para discriminar las diferentes estrellas BSGs de cada panel. 
Tabla 6.1. Parámetros estelares y del viento.

\begin{tabular}{|c|c|c|c|c|c|c|c|c|c|c|c|c|c|c|}
\hline HD & $\begin{array}{l}\mathrm{T}_{\text {ef }} \\
\mathrm{K}\end{array}$ & \multicolumn{2}{|c|}{$\begin{array}{l}\log g \log g_{e f} \\
\text { dex dex }\end{array}$} & $\beta$ & $\begin{array}{c}\dot{\mathrm{M}} \\
10^{-6} M_{\odot} / a \tilde{\mathrm{n}} O\end{array}$ & $\begin{array}{c}\mathrm{v}_{\infty} \\
\mathrm{km} / \mathrm{s}\end{array}$ & \multicolumn{4}{|c|}{$\begin{array}{l}v_{\text {micro }} V_{\text {macro }} \text { v sen } i R_{\star} \\
\mathrm{km} / \mathrm{s} \mathrm{km} / \mathrm{s} \mathrm{km} / \mathrm{s} \mathrm{R}_{\odot}\end{array}$} & $\begin{array}{c}\log \mathrm{L} / \mathrm{L} \odot \\
\operatorname{dex}\end{array}$ & $\log \mathrm{Q}$ & $\log Q_{r}$ & Referencias \\
\hline \multirow[t]{8}{*}{34085} & 12700 & 1,70 & 1,72 & 2,6 & 0,23 & 155 & 10 & 52 & 30 & 72 & 5,09 & $-12,71$ & $-9,42 \pm 0,17$ & Este estudio \\
\hline & 12000 & $\cdots$ & $\cdots$ & $\cdots$ & $0,76 / 0,94$ & 300 & $\ldots$ & $\ldots$ & 36 & 115 & 5,45 & $-12,93 /-12,83$ & $-9,21 /-9,12$ & \begin{tabular}{|l|l|} 
Chesneau et al. 2014 \\
\end{tabular} \\
\hline & 12100 & 1,75 & $\cdots$ & $\cdots$ & $\cdots$ & $\cdots$ & $\cdots$ & $\cdots$ & 25 & $\cdots$ & $\cdots$ & $\cdots$ & $\cdots$ & Firnstein \& Przybilla 2012 \\
\hline & 12500 & 1,70 & $\cdots$ & $\cdots$ & 0,34 & $230 / 350$ & 8 & 35 & 30 & 129 & 5,56 & $-13,35 /-13,08$ & $-9.63 \pm 0.17$ & Markova et al. 2008a \\
\hline & 12000 & 1,75 & $\cdots$ & $\cdots$ & $\cdots$ & $\cdots$ & $\cdots$ & 22 & 36 & 109 & 5,34 & $\cdots$ & $\cdots$ & Przybilla et al. 2006 \\
\hline & 13000 & 1,75 & $\cdots$ & $\cdots$ & $\cdots$ & $\cdots$ & $\cdots$ & 40 & $\cdots$ & $\cdots$ & 4,87 & $\cdots$ & $\cdots$ & McErlean et al. \\
\hline & 13000 & 1,60 & $\cdots$ & $\cdots$ & $\cdots$ & $400 / 600$ & $\cdots$ & 40 & $\cdots$ & $\cdots$ & $\cdots$ & $\cdots$ & $\cdots$ & Israelian et al. 1997 \\
\hline & $\cdots$ & $\ldots$ & $\cdots$ & $\cdots$ & $0,86 / 1,4$ & 530 & $\cdots$ & $\ldots$ & 33 & 135 & $\ldots$ & $-13,35 /-13,14$ & $-9,26 /-\mathbf{9 . 0 5}$ & 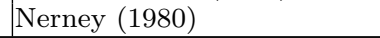 \\
\hline \multirow{7}{*}{38771} & 25000 & 2,70 & 2,76 & 1,5 & 0,14 & 1500 & 13 & 60 & 80 & 13 & 4,78 & $-13,29$ & $-8.52 \pm 0.20$ & Este estudio \\
\hline & 26000 & 3,00 & $\ldots$ & 1,1 & 1,20 & 1390 & 15 & $\ldots$ & 91 & 27,0 & 5,48 & $-12,78$ & $-8.07 \pm 0.17$ & \begin{tabular}{|l|l|} 
Searle et al. $2008 \mathrm{a}$ \\
\end{tabular} \\
\hline & 26500 & 2,70 & 2,90 & 1,5 & 0,90 & 1525 & 12,5 & $\cdots$ & 83 & 22,2 & 5,35 & $-12,84$ & $-8,07$ & $\begin{array}{ll}\text { Crowther et al. } 2006 \mathrm{a} \\
\end{array}$ \\
\hline & 27500 & 3,00 & $\cdots$ & 1,0 & 0,27 & 1350 & 10 & & 80 & 13,0 & 4,94 & $-12,94$ & $-8,24 \pm 0,13$ & Kudritzki et al. 1999 \\
\hline & 27500 & 3,00 & $\cdots$ & $\cdots$ & $\cdots$ & $\cdots$ & $\cdots$ & $\cdots$ & 80 & $\ldots$ & 4,68 & . & $\cdots$ & McErlean et al. 1999b \\
\hline & 26000 & 3,07 & 2,94 & $\cdots$ & $\cdots$ & 1870 & $\cdots$ & $\cdots$ & 81 & 28 & 5,52 & $\cdots$ & $\cdots$ & Gathier et al. 1981 \\
\hline & $\cdots$ & $\cdots$ & $\cdots$ & $\cdots$ & $0,7 / 1,1$ & $1900 / 3450$ & $\cdots$ & $\cdots$ & 82 & 30 & $\cdots$ & $-13,68 /-13,09$ & $-8,37 /-8,10$ & Nerney 1980 \\
\hline \multirow[t]{7}{*}{41117} & 19000 & 2,30 & 2,32 & 2,0 & 0,17 & 510 & 10 & 65 & 40 & 23 & 4,84 & $-12,78$ & $-8.81 \pm 0.14$ & Este trabajo \\
\hline & 20000 & 2,40 & $\ldots$ & $\cdots$ & $\cdots$ & $\cdots$ & $\cdots$ & $\cdots$ & $\cdots$ & $\cdots$ & $\cdots$ & $\cdots$ & $\cdots$ & Prugniel et al. 2011 \\
\hline & 19000 & 2,10 & 2,35 & 2,0 & 0,90 & 510 & 10 & $\cdots$ & 72 & 61,9 & 5,65 & $-12,79$ & $-8,73$ & $\begin{array}{lll}\text { Crowther et al. } 2006 \text { a } \\
\end{array}$ \\
\hline & 19500 & 2,25 & $\cdots$ & 1,0 & 0,85 & 500 & 20 & $\cdots$ & 40 & 61,7 & 5,70 & $-12,8$ & $-8.76 \pm 0.13$ & Kudritzki et al. 1999 \\
\hline & 19500 & 2,20 & $\cdots$ & $\cdots$ & $\cdots$ & $\ldots$ & $\cdots$ & 65 & $\cdots$ & $\ldots$ & 5.55 & & $\ldots$ & McErlean et a1. \\
\hline & 18500 & $\cdots$ & $\cdots$ & $\cdots$ & 1,6 & 510 & $\cdots$ & $\cdots$ & $\cdots$ & 58 & $\cdots$ & $-12,5$ & $-8,44$ & Scuderi et al. 1998 \\
\hline & $\ldots$ & $\ldots$ & $\cdots$ & $\ldots$ & 2,2 & 1000 & $\ldots$ & $\cdots$ & 36 & 63,4 & $\ldots$ & $-12,86$ & -8.36 & Nerney 1980 \\
\hline \multirow[t]{6}{*}{42087} & 16500 & 2,45 & 2,47 & 2,0 & 0,57 & 700 & 15 & 80 & 80 & 55 & 5,31 & $-13,12$ & $-8,85 \pm 0,11$ & Este trabajo \\
\hline & 18000 & 2,50 & $\cdots$ & 1,2 & 0,50 & 650 & 15 & $\cdots$ & 71 & 36,6 & 5,11 & $-12,87$ & $-8.65 \pm 0.33$ & Searle et al. 2008a \\
\hline & 19000 & 2,30 & $\cdots$ & $\cdots$ & 0,20 & $\cdots$ & $\cdots$ & $\cdots$ & $\cdots$ & $\cdots$ & 5,08 & $\cdots$ & $\cdots$ & \begin{tabular}{|l|l|} 
Benaglia et aI. & 2007 \\
\end{tabular} \\
\hline & 17150 & $\cdots$ & $\cdots$ & $\cdots$ & 0,20 & 735 & $\cdots$ & $\cdots$ & 71 & 26,0 & 4,72 & $-13,12$ & $-8,82$ & Morel et al. 2004 \\
\hline & 20500 & 2,50 & $\cdots$ & 3,0 & 0,11 & 735 & 40 & $\cdots$ & 60 & 35,2 & 5,30 & $-13,58$ & $-9.28 \pm 0.13$ & Kudritzki et al. 1999 \\
\hline & 20500 & 2,55 & $\cdots$ & $\cdots$ & $\cdots$ & $\cdots$ & $\cdots$ & $\ldots$ & 60 & $\cdots$ & 4,96 & & ... & McErlean et al. $1999 b$ \\
\hline \multirow[t]{2}{*}{47240} & 19000 & 2,40 & 2,47 & 1,0 & 0,24 & 450 & 10 & 60 & 95 & 30 & 5,03 & $-12,82$ & $-8.84 \pm 0.11$ & Este trabajo \\
\hline & 19000 & 2,40 & 2,48 & 1,5 & $0,17-0,24$ & 1000 & 15 & 55 & 94 & 27 & 4,93 & $-13,42 /-13,27$ & $-8.92 \pm 0.25 /-8.77 \pm 0.25$ & 5 Lefever et al. 2007] \\
\hline \multirow[t]{4}{*}{52089} & 23000 & 3,00 & 3,00 & 1,0 & 0,02 & 900 & 8 & 65 & 10 & 11 & 4,49 & $-13,69$ & $-9,26 \pm 0,2$ & Este trabajo \\
\hline & 20100 & 3,05 & $\cdots$ & $\cdots$ & $\cdots$ & $\cdots$ & 17 & 17 & 22 & $\cdots$ & $\cdots$ & $\cdots$ & $\cdots$ & Fraser et al. 2010 \\
\hline & 22000 & 3,20 & $\cdots$ & $\cdots$ & $\cdots$ & $\cdots$ & 15 & 20 & 32 & $\cdots$ & $\cdots$ & $\cdots$ & $\cdots$ & Lefever et al. 2010 \\
\hline & 22200 & 3,22 & $\cdots$ & $\cdots$ & $\cdots$ & $\cdots$ & 16 & $\ldots$ & $\cdots$ & $\cdots$ & $\cdots$ & $\cdots$ & $\cdots$ & Morel et al. 2008 \\
\hline \multirow[t]{3}{*}{52382} & 21500 & 2,45 & 2,48 & 2,2 & 0,24 & 1000 & 10 & 65 & 55 & 21 & 4,94 & $-13,10$ & $-8,60 \pm 0,13$ & Este trabajo \\
\hline & 23000 & $\cdots$ & 2,71 & $\cdots$ & $\cdots$ & $\cdots$ & 20 & 53 & 56 & $\cdots$ & $\cdots$ & $\ldots$ & $\cdots$ & \begin{tabular}{|l|l|} 
Lefever et al. 2010 \\
\end{tabular} \\
\hline & 20800 & $\cdots$ & $\cdots$ & $\cdots$ & 0,49 & 1200 & & $\ldots$ & & $\cdots$ & $\cdots$ & $\cdots$ & & Krtička \& Kübát 2001a \\
\hline \multirow[t]{6}{*}{53138} & 18000 & 2,25 & 2,26 & 2,0 & $0,2 / 0,24$ & $600 / 450$ & 10 & 60 & 40 & 46 & 5,31 & $-13,36 /-13,09$ & $-9,19 \pm 0,11 /-9,11 \pm 0,11$ & Este trabajo \\
\hline & 15400 & 2,15 & $\cdots$ & $\cdots$ & $\cdots$ & $\cdots$ & 18 & 23 & 35 & $\cdots$ & $\cdots$ & $\cdots$ & $\cdots$ & Fraser et al. 2010 \\
\hline & 16500 & 2,25 & $\cdots$ & 1,2 & 0,45 & 500 & 20 & $\cdots$ & 58 & 54,7 & 5,30 & $-13,00$ & $-8,95 \pm 0,17$ & Searle et al. 2008a \\
\hline & 17000 & 2,15 & 2,16 & 2,5 & $0,21 / 0,31$ & 490 & 15 & 45 & 38 & 50 & 5,27 & $-13,20$ & $-9,23 \pm 0,25 /-9,06 \pm 0,25$ & 6 Lefever et a. 2007 \\
\hline & 15500 & 1,75 & 2,05 & 2,0 & 0,36 & 865 & 20 & $\cdots$ & 58 & 65 & 5,34 & $-13,57$ & $-9,16$ & Crowther et al. 2006 a \\
\hline & 18500 & 2,30 & & 2,5 & 0,095 & 620 & & 40 & 60 & 39,6 & 5,22 & & $-9.42 \pm 0.13$ & Kudritzki et al. 1999 \\
\hline
\end{tabular}


Tabla 6.1. Continuación.

\begin{tabular}{|c|c|c|c|c|c|c|c|c|c|c|c|c|c|c|}
\hline \multirow[t]{3}{*}{$\overline{\mathrm{HD}}$} & \multicolumn{3}{|c|}{$\begin{array}{ll}\mathrm{T}_{\text {ef }} & \log \mathrm{g} \log \mathrm{g}_{\mathrm{ef}} \\
\mathrm{K} & \text { dex dex }\end{array}$} & \multirow[t]{2}{*}{$\beta$} & $\begin{array}{ll} & \dot{M} \\
10^{-6} & M_{\odot} / a \tilde{n} o \\
\end{array}$ & \multirow[t]{2}{*}{$\begin{array}{c}\mathrm{v}_{\infty} \\
\mathrm{km} / \mathrm{s}\end{array}$} & \multicolumn{4}{|c|}{$\begin{array}{l}\mathrm{v}_{\text {micro }} \mathrm{V}_{\text {macro }} \mathrm{v} \operatorname{sen} i \mathrm{v}_{\star} \\
\mathrm{km} / \mathrm{s} \mathrm{km} / \mathrm{s} \mathrm{km} / \mathrm{s}_{\odot}\end{array}$} & \multirow{3}{*}{$\begin{array}{c}\begin{array}{c}\log \mathrm{L} / \mathrm{L} \odot \\
\operatorname{dex}\end{array} \\
5,04 \\
\ldots\end{array}$} & $\log \mathrm{Q}$ & 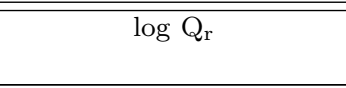 & \multirow{3}{*}{\begin{tabular}{|l|}
\multicolumn{2}{|l|}{ Referencias } \\
McErlean et al. 1999b \\
\end{tabular}} \\
\hline & 18500 & 2,35 & & & & & & & 55 & & & & $\ldots$ & \\
\hline & $\ldots$ & $\ldots$ & $\ldots$ & $\ldots$ & 1,7 & 580 & $\ldots$ & $\ldots$ & $\ldots$ & 59,1 & & $\ldots$ & -8.43 & \\
\hline \multirow[t]{5}{*}{58350} & 15500 & 2,00 & 2,02 & 3,0 & $0,116-0,15$ & $175-233$ & 11 & $50 / 70$ & 40 & 54 & $5,13-5,24$ & $1-12,9 /-12,97$ & $-9,53 \pm 0,13 /-9,42 \pm 0,13$ & Este trabajo \\
\hline & 14500 & 2,10 & $\ldots$ & . & & $\ldots$ & 18 & 25 & 32 & & & & r & Fraser et al. 2010 \\
\hline & 15000 & 2,13 & $\ldots$ & 1,0 & 0,70 & 320 & 20 & $\cdots$ & 50 & 57,3 & 5,18 & $-12,55$ & $-8.79 \pm 0.23$ & Searle et al. 2008 \\
\hline & 13500 & 1,75 & 1,77 & 2,5 & 0,14 & 250 & 12 & 40 & 37 & 65 & 5,10 & $-13,17$ & $-9.57 \pm 0.25$ & Lefever et al. \\
\hline & 16000 & 2.10 & $\cdots$ & , & .. & $\ldots$ & $\ldots$ & 40 & $\ldots$ & & 5,36 & .. & .. & McErlean et al. |1999b \\
\hline \multirow[t]{4}{*}{64760} & 23000 & 2,90 & 3,09 & 0,5 & 0,42 & 1500 & 15 & 100 & 230 & 12 & 4,57 & $-12,76$ & $-8.00 \pm 0.13$ & Este trabajo \\
\hline & 26000 & 3,25 & $\ldots$ & $\cdots$ & $\ldots$ & $\ldots$ & 22 & $\cdots$ & 255 & $\ldots$ & $\ldots$ & $\ldots$ & $\ldots$ & Fraser et al. 2010 \\
\hline & 28000 & 3,38 & $\ldots$ & 1,0 & 1,10 & 1600 & 15 & $\ldots$ & 265 & 23,3 & 5,48 & $-12,82$ & $-8,01 \pm 0,17$ & Searle et al. $2008 \mathrm{a}$ \\
\hline & 24000 & 3,20 & 3,27 & 0,8 & 0,42 & 1400 & 14 & $\ldots$ & 230 & 24 & 5,23 & $-13,16$ & $-8.45 \pm 0.25$ & Lefever et al. \\
\hline \multirow[t]{2}{*}{74371} & 13700 & 1,80 & 1,81 & 2 & 0,28 & 200 & 10 & 60 & 30 & 73 & 5,23 & $-12,8$ & $-9,35 \pm 0,11$ & Este trabajo \\
\hline & 13400 & 1,90 & & 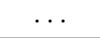 & 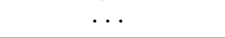 & $\cdots$ & $\cdots$ & 20 & 30 & 31 & & & $\cdots$ & Fraser et al. 2010 \\
\hline \multirow[t]{3}{*}{75149} & 16000 & 2,10 & 2,11 & 2,5 & $0,09 / 0,25$ & $400 / 350$ & $9 / 17$ & $55 / 60$ & 40 & 61 & 5,35 & $-13,63 /-13,1$ & $-9.72 \pm 0.15 /-9.28 \pm 0.15$ & Este trabajo \\
\hline & 15900 & 2,20 & $\ldots$ & $\ldots$ & $\ldots$ & $\ldots$ & 20 & 34 & 30 & $\ldots$ & $\cdots$ & $\ldots$ & $\cdots$ & Fraser et al. 2010 \\
\hline & 16000 & 2,05 & 2,06 & 2,5 & 0,10 & 500 & 15 & 60 & 30 & 39 & 4,95 & $-13,43$ & $-9,39 \pm 0,25$ & Lefever et al. $\mid 2007$ \\
\hline \multirow[t]{5}{*}{79186} & 15800 & 2,0 & 2,02 & 3,3 & 0,40 & 400 & 11 & 53 & 40 & 61 & 5,33 & $-12,98$ & $-9,08 \pm 0,09$ & Este trabajo \\
\hline & 15100 & 2,0 & . & $\ldots$ & & $\ldots$ & 14 & 36 & 39 & & $\ldots$ & $\ldots$ & $\cdots$ & Fraser et al. 2010 \\
\hline & 15000 & $\cdots$ & $\cdots$ & $\cdots$ & 0,80 & 435 & $\ldots$ & $\cdots$ & $\ldots$ & $\ldots$ & $\ldots$ & $\ldots$ & $\ldots$ & Prinja \& Massa 2010 \\
\hline & 13600 & $\ldots$ & $\ldots$ & $\ldots$ & 0,66 & 450 & $\ldots$ & $\ldots$ & $\ldots$ & 62,4 & $\ldots$ & $-12,85$ & $-8,87 \pm 0,09$ & Krticka \& Kubát||2001a) \\
\hline & 13900 & $\ldots$ & $\ldots$ & $\ldots$ & $\ldots$ & $\ldots$ & ... & $\ldots$ & 45 & 81 & $\ldots$ & $\ldots$ & $\ldots$ & 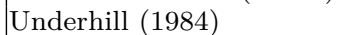 \\
\hline \multirow{3}{*}{80077} & 17700 & 2,20 & 2,20 & $3-3,2$ & 5,4 & $150 / 200$ & 10 & 60 & 10 & 195 & 6,53 & $-11,97 / 12,15$ & $-8.70 \pm 0.11$ & Este trabajo \\
\hline & 17000 & 2,00 & $\ldots$ & $\ldots$ & 5,00 & 140 & $\ldots$ & & $\ldots$ & 162 & 6,3 & $-11,83$ & $-8.62 \pm 0.65$ & Carpay et al. \\
\hline & 18500 & 2,25 & $\ldots$ & $\ldots$ & 1,70 & 140 & $\ldots$ & $\ldots$ & $\ldots$ & $\ldots$ & 5,4 & $\ldots$ & $\ldots$ & Benaglia et al. \\
\hline \multirow{4}{*}{92964} & 18000 & 2,20 & 2,21 & 2,0 & 0,49 & 370 & 11 & 40 & 45 & 70 & 5,67 & $-12,93$ & $-9,08 \pm 0,09$ & Este trabajo \\
\hline & 15600 & 2,00 & , & ... & & $\ldots$ & 22 & 28 & 36 & $\ldots$ & & $\ldots$ & & Fraser et al. 2010 \\
\hline & 18000 & 2,10 & 2,10 & 3,0 & $0,25 / 0,28$ & 520 & 15 & 50 & 31 & 48 & 5,33 & $-13,14 /-13,19$ & $-9.07 \pm 0.25$ & \begin{tabular}{|l|l|} 
Lefever et al. & 2007 \\
\end{tabular} \\
\hline & 17400 & $\ldots$ & $\ldots$ & $\ldots$ & 0,11 & $530 / 807$ & $\ldots$ & $\ldots$ & $\ldots$ & 68 & & $-13,79 /-14,07$ & -9.71 & Krticka \& Kubát|2001a \\
\hline \multirow[t]{2}{*}{99953} & 19000 & 2,30 & 2,33 & 2,0 & $0,08 / 0,22$ & $250 / 700$ & 18 & 50 & 50 & 25 & 4,87 & $-12,79 /-13,02$ & $-9.19 \pm 0.11 /-8.75 \pm 0.11$ & Este trabajo \\
\hline & 16800 & 2,15 & $\ldots$ & $\ldots$ & $\ldots$ & $\ldots$ & 22 & 37 & 49 & $\ldots$ & . & & $\ldots$ & Fraser et al. \\
\hline \multirow{3}{*}{111973} & 16500 & 2,10 & 2,11 & 2,0 & $0,14 / 0,21$ & 350 & 12 & $\sim 60$ & 35 & 48 & 5,16 & $-13,19 /-13,02$ & $-9,38 \pm 0,09 /-9,20 \pm 0,09$ & Este trabajo \\
\hline & 16000 & 2,30 & & $\ldots$ & ... & $\ldots$ & 19 & 28 & 36 & $\ldots$ & $\ldots$ & $\ldots$ & $\ldots$ & Fraser et al. 2010 \\
\hline & 16500 & & .. & 0,5 & ... & 520 & $\ldots$ & & $\ldots$ & $\ldots$ & $\ldots$ & $\ldots$ & $\ldots$ & Prinja \& Massa \\
\hline \multirow[t]{3}{*}{115842} & 25500 & 2,75 & 2,76 & 2,5 & 1,8 & 1700 & 10 & 120 & 70 & 35 & 5,68 & $-12,91$ & $-8.06 \pm 0.13$ & Este trabajo \\
\hline & 24800 & 2,75 & $\ldots$ & $\cdots$ & $\ldots$ & $\ldots$ & 14 & 66 & 39 & $\ldots$ & $\ldots$ & $\ldots$ & $\ldots$ & Fraser et al. 2010 \\
\hline & 25500 & 2,65 & 2,85 & 1,5 & 2,0 & 1180 & 10 & .. & 84 & 34,2 & 5,65 & $-12,61$ & -8.00 & Crowther et al. \\
\hline \multirow[t]{4}{*}{148688} & 21000 & 2,45 & 2,47 & 2,5 & 1,15 & 1200 & 11 & 65 & 50 & 31 & 5,23 & $-12,8$ & $-8,18 \pm 0,15$ & Este trabajo \\
\hline & 20700 & 2,45 & $\ldots$ & $\ldots$ & $\ldots$ & $\ldots$ & 16 & 44 & 48 & $\ldots$ & $\ldots$ & $\ldots$ & $\ldots$ & Fraser et al. \\
\hline & 21000 & 2,50 & 2,5 & 3,0 & $1,10 / 1,40$ & 1200 & 15 & 40 & 50 & 42 & 5,49 & $-12,90 /-13,01$ & $-8,29 \pm 0,25$ & Lefever et al. $\mid 2007$ \\
\hline & 22000 & 2,40 & 2,60 & 2,0 & 1,75 & 725 & 15 & & 72 & 36,7 & 5,45 & $-12,39$ & -8.10 & Crowther et al. \\
\hline
\end{tabular}



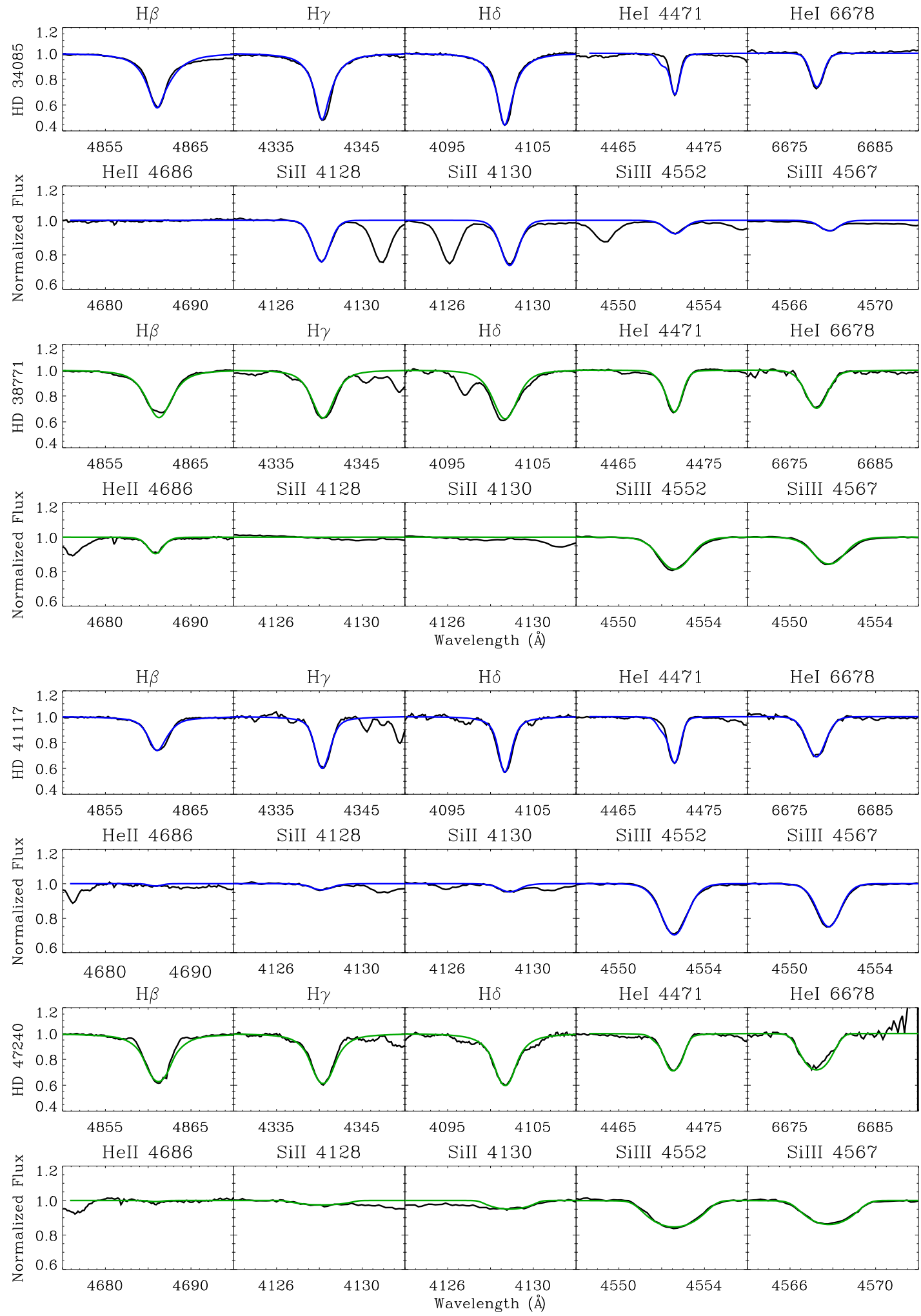

Figura 6.1. HD 34 085, HD 38771 , HD 41 117, y HD 47240. 

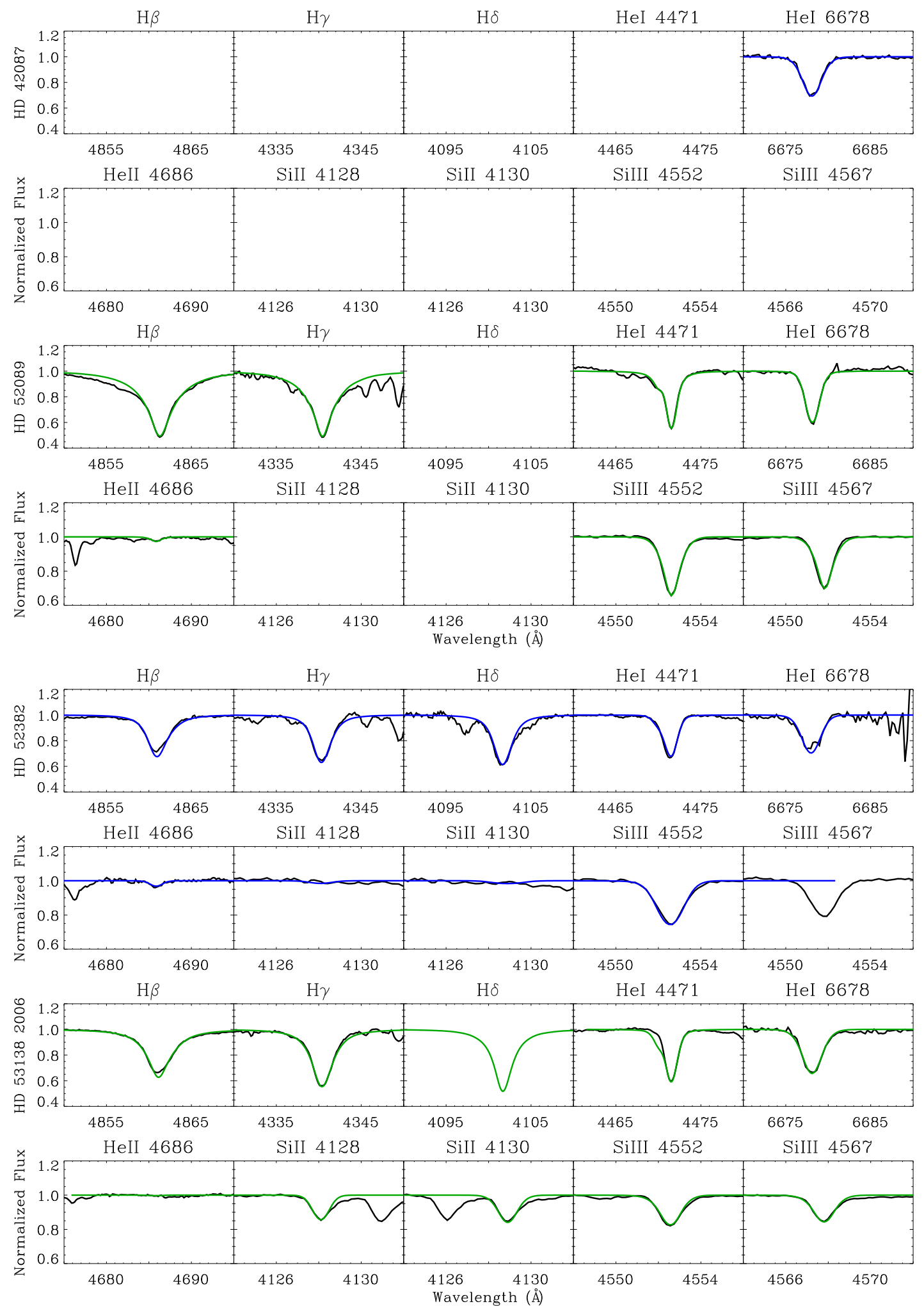

Figura 6.2. HD 42 087, HD 52 089, HD 52 382, y HD 53138. 

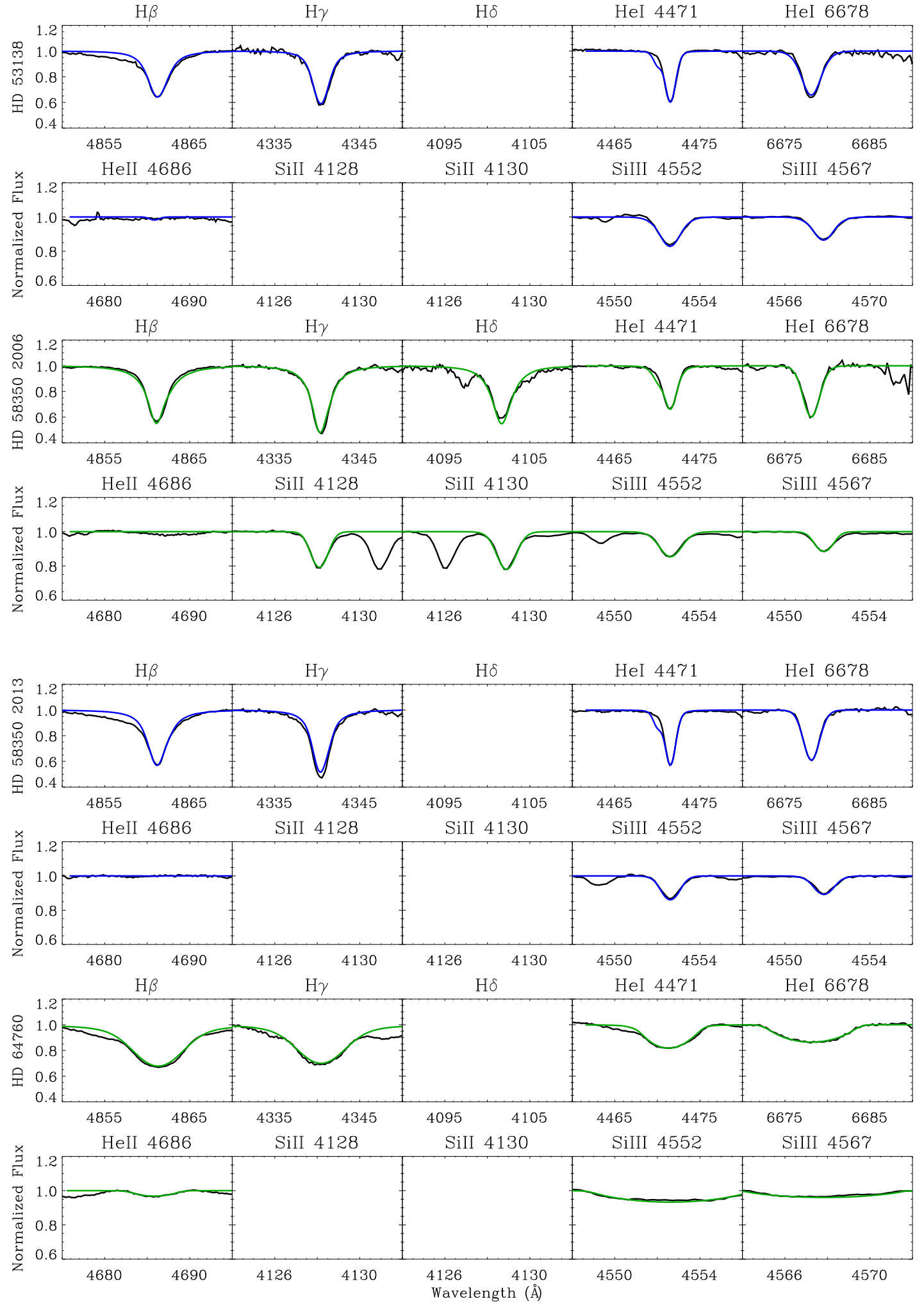

Figura 6.3. HD 53138 , HD 58350 y HD 64760. 

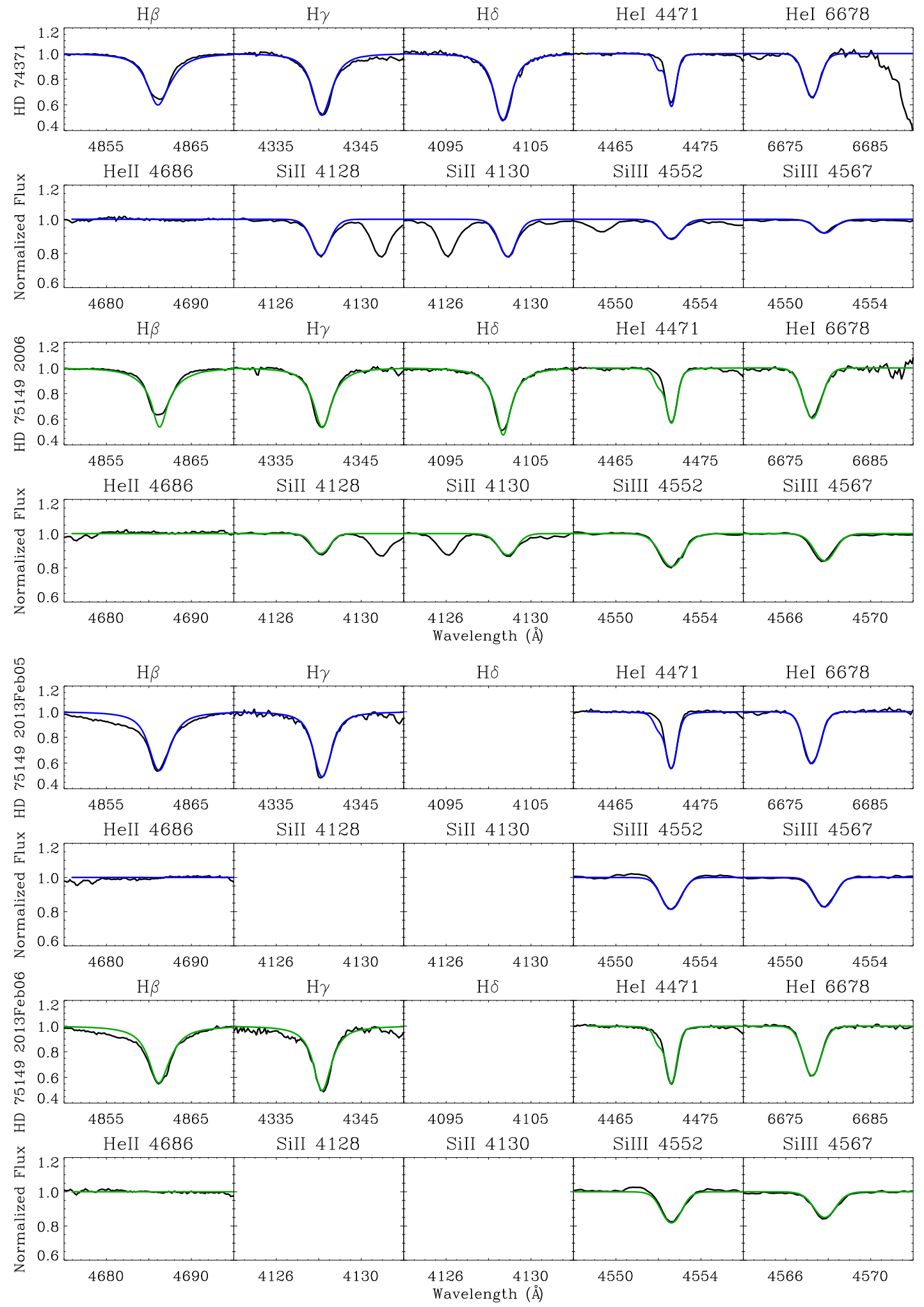

Figura 6.4. HD 74371 y HD 75149. 

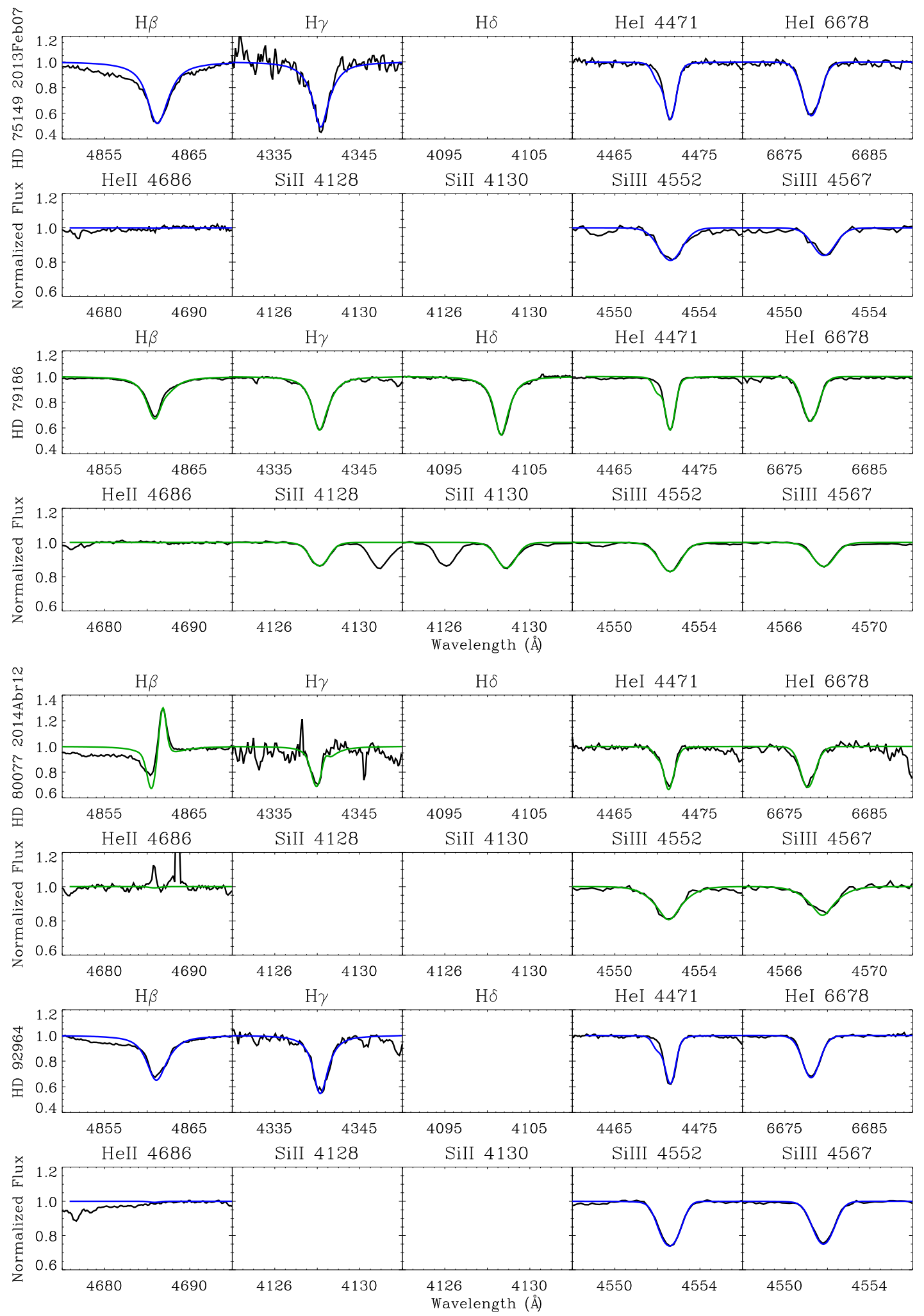

Figura 6.5. HD 75 149, HD 79 186, HD 92964 y HD 80077. 

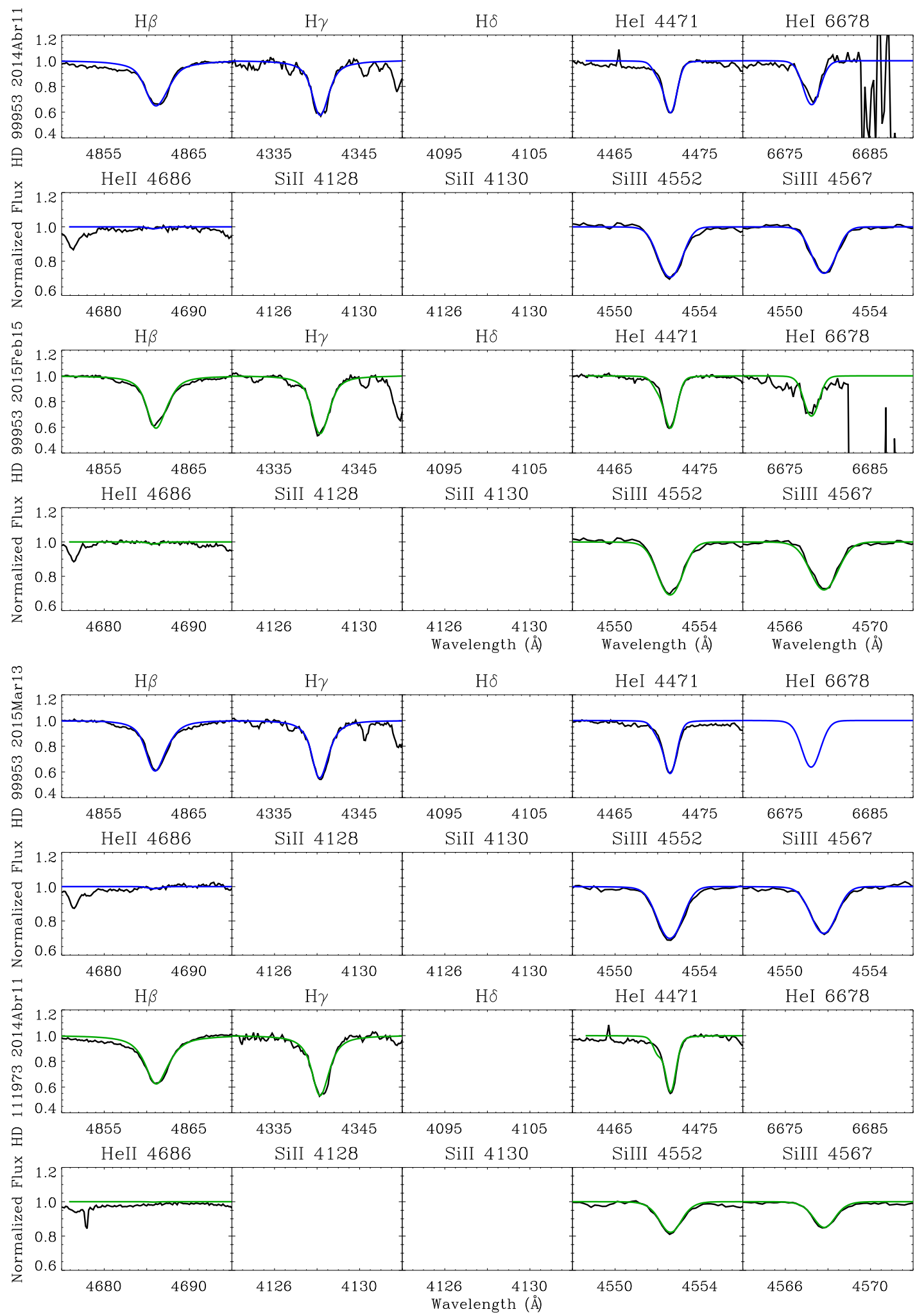

Figura 6.6. HD 99953 y HD 111973. 

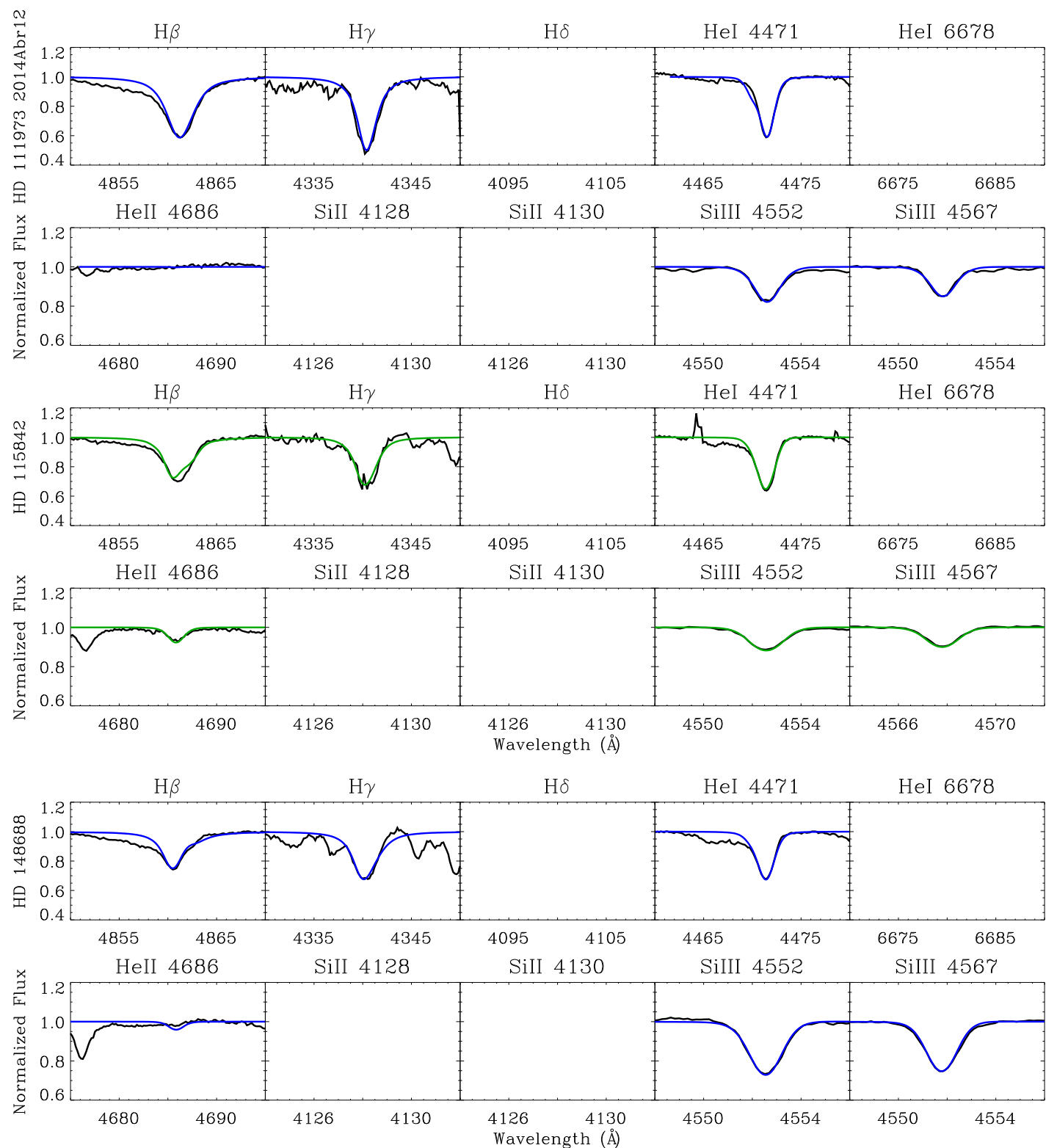

Figura 6.7. HD 111 973, HD 115842 y HD 148688. 


\section{Apéndice B}

\subsection{Búsqueda de pulsaciones: análisis de momentos}

Como 55 Cygni ocupa un lugar en el diagrama HR, donde diferentes modos de pulsación parecen estar excitados Saio et al. 2006, y puesto que además es una candidata a variable $\alpha$ cygni, es natural esperar que hayan pulsaciones en su superficie. La fuerte variabilidad de $\mathrm{H}_{\alpha}$ (sugiriendo pérdida de masa variable) puede indicar que los cambios observados en el viento puedan estar conectados a pulsaciones detectadas en las líneas fotosféricas.

Ante la ausencia de curvas de luz tomadas en lapsos largos de tiempo, la presencia de pulsaciones también puede ser estudiada analizando las variaciones fotosféricas de determinadas líneas espectrales, siempre y cuando sean de alta resolución. Dadas las caracerísticas de nuestras observaciones, decidimos usar para hacer este tratamiento la línea de He I $\lambda 6678$ Å. El análisis consiste en aplicar el método de los momentos Aerts et al. 1992 North \& Paltani 1994 el cual es ideal ya que permite distinguir variaciones causadas por manchas (spots) o pulsaciones.

El primer momento corresponde a la velocidad radial de la línea. Obtenemos que este parámetro varía entre $+5 \mathrm{~km} / \mathrm{s}$ y $-21 \mathrm{~km} / \mathrm{s}$ entre agosto de 2009 y octubre de 2013 , como muestra la fig. 6.8. También puede verse que la variación no es simétrica con respecto a velocidad 0, pero esto se debe a que los datos no están corregidos por la velocidad radial intrínseca, dato que se desprende del gráfico y toma un valor de $\sim 8 \mathrm{~km} / \mathrm{s}$. Por lo tanto, obtenemos una amplitud de variación en la velocidad radial de $\pm 13 \mathrm{~km} / \mathrm{s}$. Los próximos análisis están corregidos por dicho valor.

Las variaciones de los momentos uno y tres se muestran en los paneles medio e inferior de la fig. 6.9. Para una mejor visibilidad del gráfico, éste está limitado a un período de 25 días, entre del 19/7/2013 y 12/8/2013. El tercer momento, el cual describe la asimetría de la línea, muestra la misma variabilidad que la velocidad radial, lo cual es característico para pulsaciones estelares Aerts et al. 1992). Comportamientos similares son observados en otras épocas.

Por úlimo, una prueba adicional para determinar la presencia de pulsaciones en la fotosfera de $55 \mathrm{Cyg}$, es comparar los diferentes momentos de diferentes elementos. Por lo tanto medimos los momentos de las líneas CII $\lambda 6578 \AA$ y SiII $\lambda 6347 \AA$ restringidos a las noches del 21-22/7/2013, la cuales tienen la mayor S/N. Los resultados, en conjunto con la línea del HeI son mostrados en la fig. 6.10, donde puede verse claramente cómo todos los elementos tienen el mismo patrón de variación. Tal comportamiento excluye la presencia 


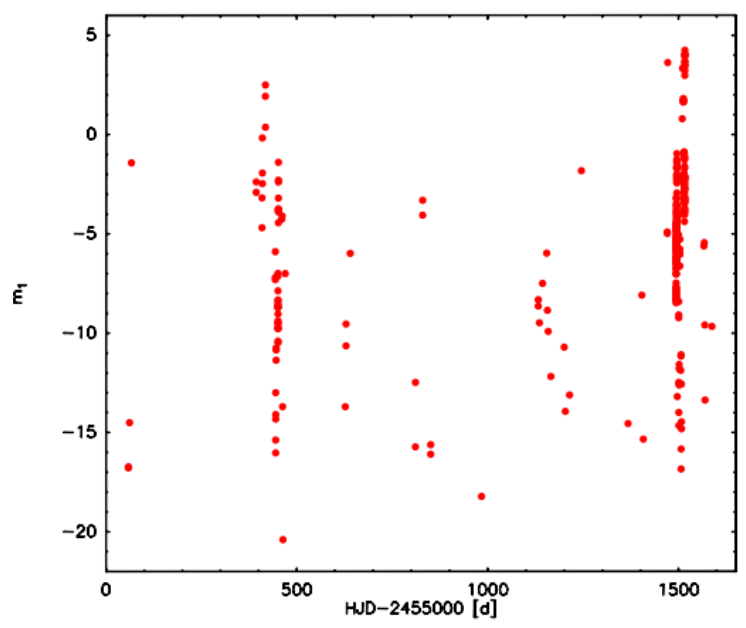

Figura 6.8. Variación en el primer momento (velocidad radial, sin corrección por velocidad sistémica) de HeI $\lambda 6678 \AA$ respecto al tiempo.

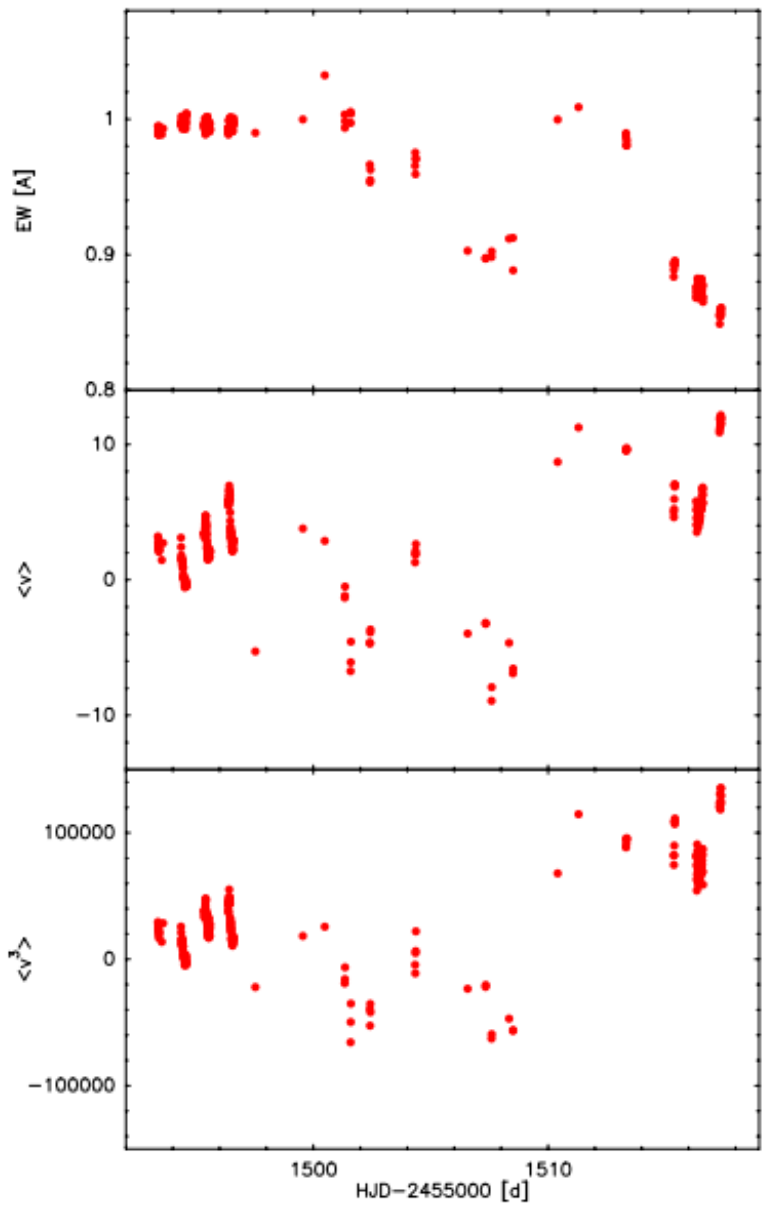

Figura 6.9. Variación del ancho equivalente (arriba), el primer (velocidad radial, al medio) y tercer momento (asimetría, abajo) de He I $\lambda 6678 \AA$ para un período de 25 días. 


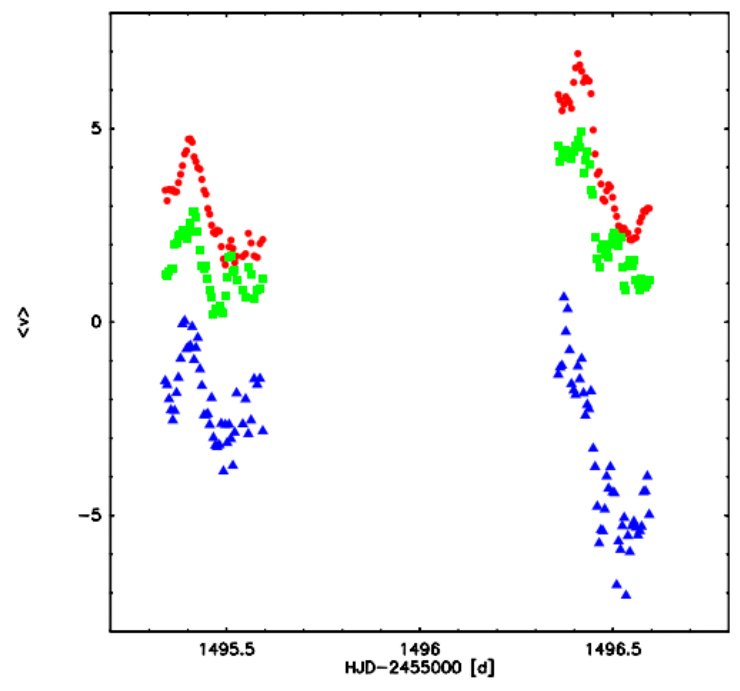

Figura 6.10. Variación del primer momento de HeI $\lambda 6678 \AA$ (puntos rojos), CII $\lambda 6578 \AA$ (cuadrados verdes) y SiII $\lambda 6347 \AA$ (triángulos azules)

de manchas, y como 55 Cyg no tiene anomalías químicas en He, este hecho termina de confirmar la presencia de pulsaciones.

\subsection{Análisis de frecuencias}

Los resultados anteriores confirmaron la presencia de pulsaciones como causantes de las variaciones en los perfiles de He y otras líneas metálicas. El siguiente paso consiste analizar las curvas de velocidad radial en búsqueda de períodos. En nuestro caso, el análisis es similar al que describió Saio et al. (2006). El primer paso consiste en hacer el periodograma de Lomb-Scargle Scargle 1982 (fig. 6.11). El periodograma parece no mostrar un período claro, pero en su lugar hay varios picos significativos. Este comportamiento suele verse en las variables $\alpha$ Cygni (Lucy 1976). Usando funciones de ajustes que sean series sinusoidales, el proceso arrojó 19 frecuencias con un nivel de confiabilidad del $99 \%$, mostradas en la tabla 6.2 


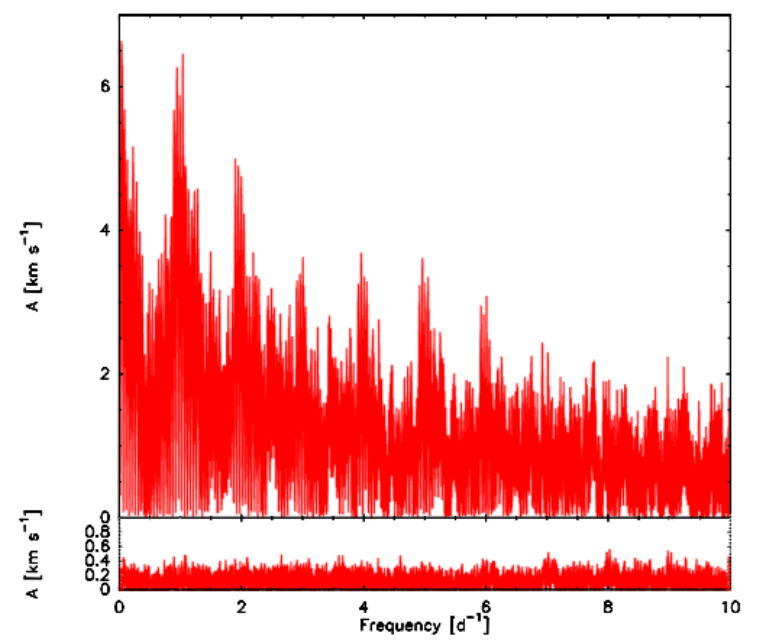

Figura 6.11. Periodograma de Lomb-Scargle de la velocidad radial original (arriba) y los datos "limpios" de las 19 sinusoides identificadas.

Tabla 6.2. Periodicidades de 55 Cyg. Las fases están referenciadas respeto a HJD = $2455059.00 \mathrm{~d}$.

\begin{tabular}{ccccccc}
\hline \hline No. & $f \begin{array}{c}d f \times 10^{8} \\
\left(\text { dia }^{-1}\right)\end{array}$ & \multicolumn{2}{c}{$\begin{array}{c}A \\
\left(\mathrm{~km} \mathrm{~s}^{-1}\right)\end{array}$} & $\begin{array}{c}\text { c } \\
(\mathrm{rad})\end{array}$ \\
\hline$f_{1}$ & 0.044366 & 0.03 & 4.39 & 0.03 & 2.43 & 0.01 \\
$f_{2}$ & 0.995570 & 0.03 & 3.31 & 0.04 & 3.71 & 0.02 \\
$f_{3}$ & 0.235716 & 0.07 & 2.54 & 0.03 & 3.44 & 0.06 \\
$f_{4}$ & 0.974983 & 0.17 & 1.42 & 0.03 & 5.40 & 0.09 \\
$f_{5}$ & 1.278593 & 0.18 & 1.37 & 0.03 & 3.35 & 0.10 \\
$f_{6}$ & 2.400132 & 0.17 & 1.54 & 0.02 & 1.21 & 0.11 \\
$f_{7}$ & 0.506733 & 0.22 & 1.30 & 0.07 & 5.55 & 0.13 \\
$f_{8}$ & 4.163120 & 0.26 & 0.87 & 0.01 & 5.78 & 0.17 \\
$f_{9}$ & 6.994100 & 0.21 & 0.95 & 0.01 & 1.76 & 0.14 \\
$f_{10}$ & 3.104664 & 0.18 & 1.18 & 0.02 & 5.29 & 0.10 \\
$f_{11}$ & 0.188665 & 0.42 & 1.09 & 0.04 & 3.43 & 0.26 \\
$f_{12}$ & 1.462843 & 0.40 & 1.06 & 0.03 & 2.77 & 0.25 \\
$f_{13}$ & 0.296804 & 0.24 & 1.11 & 0.03 & 3.31 & 0.14 \\
$f_{14}$ & 0.652575 & 1.21 & 0.79 & 0.03 & 1.24 & 0.77 \\
$f_{15}$ & 1.181013 & 1.55 & 0.55 & 0.04 & 6.03 & 1.00 \\
$f_{16}$ & 0.617903 & 0.30 & 1.00 & 0.02 & 0.04 & 0.17 \\
$f_{17}$ & 2.593881 & 0.45 & 0.75 & 0.02 & 5.46 & 0.28 \\
$f_{18}$ & 9.003491 & 0.52 & 0.54 & 0.01 & 2.09 & 0.34 \\
$f_{19}$ & 3.780456 & 1.10 & 0.51 & 0.02 & 4.94 & 0.79 \\
\hline
\end{tabular}




\section{Bibliografía}

Abbott, D. C. 1982, ApJ, 259, 282

Abt, H. A., Levato, H., \& Grosso, M. 2002, ApJ, 573, 359

Aerts, C., Christensen-Dalsgaard, J., \& Kurtz, D. W. 2010a, Asteroseismology

Aerts, C., de Pauw, M., \& Waelkens, C. 1992, A\&A, 266, 294

Aerts, C., Lefever, K., Baglin, A., et al. 2010b, A\&A, 513, L11

Aerts, C., Puls, J., Godart, M., \& Dupret, M.-A. 2009a, A\&A, 508, 409

Aerts, C., Puls, J., Godart, M., \& Dupret, M.-A. 2009b, Communications in Asteroseismology, 158, 66

Aerts, C., Simón-Díaz, S., Catala, C., et al. 2013, A\&A, 557, A114

Arcos, C., Jones, C. E., Sigut, T. A. A., Kanaan, S., \& Curé, M. 2017, ApJ, 842, 48

Asplund, M., Grevesse, N., \& Sauval, A. J. 2005, in Astronomical Society of the Pacific Conference Series, Vol. 336, Cosmic Abundances as Records of Stellar Evolution and Nucleosynthesis, ed. T. G. Barnes, III \& F. N. Bash, 25

Astraatmadja, T. L. \& Bailer-Jones, C. A. L. 2016, ApJ, 833, 119

Barbier, D. \& Chalonge, D. 1941, Annales d'Astrophysique, 4, 30

Barlow, M. J. \& Cohen, M. 1977, ApJ, 213, 737

Bates, B., Catney, M., Gilheany, S., \& Halliwell, D. 1988, in ESA Special Publication, Vol. 281, ESA Special Publication

Becker, S. R. \& Butler, K. 1990, A\&A, 235, 326

Benaglia, P., Vink, J. S., Martí, J., et al. 2007, A\&A, 467, 1265

Berghoefer, T. W., Schmitt, J. H. M. M., Danner, R., \& Cassinelli, J. P. 1997, A\&A, 322, 167 
Bertelli, G., Nasi, E., Girardi, L., \& Marigo, P. 2009, A\&A, 508, 355

Bouret, J.-C., Lanz, T., \& Hillier, D. J. 2005, A\&A, 438, 301

Brott, I., de Mink, S. E., Cantiello, M., et al. 2011, A\&A, 530, A115

Burki, G., Maeder, A., \& Rufener, F. 1978, A\&A, 65, 363

Cantiello, M., Langer, N., Brott, I., et al. 2009, Communications in Asteroseismology, 158, 61

Carlberg, R. G. 1980, ApJ, 241, 1131

Carpay, J., de Jager, C., \& Nieuwenhuijzen, H. 1991, A\&A, 248, 475

Carpay, J., de Jager, C., Nieuwenhuijzen, H., \& Moffat, A. 1989, A\&A, 216, 143

Castor, J. I., Abbott, D. C., \& Klein, R. I. 1975, ApJ, 195, 157

Chalonge, D. \& Divan, L. 1973, A\&A, 23, 69

Chesneau, O., Dessart, L., Mourard, D., et al. 2010, A\&A, 521, A5

Chesneau, O., Kaufer, A., Stahl, O., et al. 2014, A\&A, 566, A125

Chieffi, A. \& Limongi, M. 2013, ApJ, 764, 21

Chini, R., Hoffmeister, V. H., Nasseri, A., Stahl, O., \& Zinnecker, H. 2012, MNRAS, 424, 1925

Cidale, L. S. \& Ringuelet, A. E. 1993, ApJ, 411, 874

Cohen, D. H., Cooper, R. G., Macfarlane, J. J., et al. 1996, ApJ, 460, 506

Cohen, D. H., Leutenegger, M. A., Wollman, E. E., et al. 2010, MNRAS, 405, 2391

Cramer, N. 1999, , 43, 343

Cranmer, S. R. \& Owocki, S. P. 1996, ApJ, 462, 469

Crowther, P. A., Lennon, D. J., \& Walborn, N. R. 2006a, A\&A, 446, 279

Crowther, P. A., Lennon, D. J., \& Walborn, N. R. 2006b, A\&A, 446, 279

Curé, M. 2004, ApJ, 614, 929

Curé, M., Cidale, L., \& Granada, A. 2011, ApJ, 737, 18

Curé, M., Rial, D. F., \& Cidale, L. 2005, A\&A, 437, 929

Day, R. W. \& Warner, B. 1975, MNRAS, 173, 419 
Decressin, T., Mathis, S., Palacios, A., et al. 2009, A\&A, 495, 271

Dessart, L. \& Owocki, S. P. 2005, A\&A, 437, 657

Ebbets, D. 1982, ApJS, 48, 399

Ekström, S., Georgy, C., Eggenberger, P., et al. 2012, A\&A, 537, A146

Evans, C. J., Lennon, D. J., Trundle, C., Heap, S. R., \& Lindler, D. J. 2004, ApJ, 607, 451

Feldmeier, A. 1995, A\&A, 299, 523

Firnstein, M. \& Przybilla, N. 2006, in International Symposium on Nuclear Astrophysics - Nuclei in the Cosmos, 95.1

Firnstein, M. \& Przybilla, N. 2012, A\&A, 543, A80

Flower, P. J. 1996, ApJ, 469, 355

Fossati, L., Castro, N., Morel, T., et al. 2015, A\&A, 574, A20

Fraser, M., Dufton, P. L., Hunter, I., \& Ryans, R. S. I. 2010, MNRAS, 404, 1306

Frémat, Y., Zorec, J., Hubert, A., \& Floquet, M. 2005, A\&A, 440, 305

Friend, D. B. \& Abbott, D. C. 1986, ApJ, 311, 701

Fullerton, A. W., Massa, D. L., \& Prinja, R. K. 2004, in Bulletin of the American Astronomical Society, Vol. 36, American Astronomical Society Meeting Abstracts, 1425

Fullerton, A. W., Massa, D. L., \& Prinja, R. K. 2006, ApJ, 637, 1025

Garcia, M., Herrero, A., Najarro, F., et al. 2017, in IAU Symposium, Vol. 329, The Lives and Death-Throes of Massive Stars, ed. J. J. Eldridge, J. C. Bray, L. A. S. McClelland, \& L. Xiao, 313-321

Garmany, C. D., Olson, G. L., van Steenberg, M. E., \& Conti, P. S. 1981, ApJ, 250, 660

Gathier, R., Lamers, H. J. G. L. M., \& Snow, T. P. 1981, ApJ, 247, 173

Gautschy, A. \& Glatzel, W. 1990, MNRAS, 245, 597

Georgy, C., Saio, H., \& Meynet, G. 2014, MNRAS, 439, L6

Gies, D. R. \& Lambert, D. L. 1992a, ApJ, 387, 673

Gies, D. R. \& Lambert, D. L. 1992b, ApJ, 387, 673

Glatzel, W. 1994, MNRAS, 271 
Glatzel, W. \& Kiriakidis, M. 1993, MNRAS, 263, 375

Godart, M., Simón-Díaz, S., Herrero, A., et al. 2017, A\&A, 597, A23

Gray, D. F. 1976, The observation and analysis of stellar photospheres

Grevesse, N. \& Sauval, A. J. 1998, , 85, 161

Güdel, M. \& Nazé, Y. 2009, A\&AR, 17, 309

Hanbury Brown, R., Davis, J., \& Allen, L. R. 1974, MNRAS, 167, 121

Harnden, Jr., F. R., Branduardi, G., Gorenstein, P., et al. 1979, ApJL, 234, L51

Henrichs, H. F., Neiner, C., \& Geers, V. C. 2003, in IAU Symposium, Vol. 212, A Massive Star Odyssey: From Main Sequence to Supernova, ed. K. van der Hucht, A. Herrero, \& C. Esteban, 202

Herrero, A., Puls, J., \& Najarro, F. 2002, A\&A, 396, 949

Hillier, D. J. \& Miller, D. L. 1998, ApJ, 496, 407

Hoffleit, D. \& Jaschek, C. 1991, The Bright star catalogue

Howarth, I. D., Siebert, K. W., Hussain, G. A. J., \& Prinja, R. K. 1997a, MNRAS, 284, 265

Howarth, I. D., Siebert, K. W., Hussain, G. A. J., \& Prinja, R. K. 1997b, MNRAS, 284, 265

Hubeny, I. \& Lanz, T. 1995a, ApJ, 439, 875

Hubeny, I. \& Lanz, T. 1995b, ApJ, 439, 875

Hubeny, I. \& Lanz, T. 2017, ArXiv e-prints [[arXiv]1706.01859]

Humphreys, R. M. 1978, ApJS, 38, 309

Hutchings, J. B. 1970, MNRAS, 147, 161

Iben, I. \& Renzini, A. 1984, , 105, 329

Israelian, G., Chentsov, E., \& Musaev, F. 1997, MNRAS, 290, 521

Jurkić, T., Sarta Deković, M., Dominis Prester, D., \& Kotnik-Karuza, D. 2011, Ap\&SS, 335,113

Kaper, L., Henrichs, H. F., Fullerton, A. W., et al. 1997, A\&A, 327, 281

Kaper, L., Henrichs, H. F., Nichols, J. S., \& Telting, J. H. 1999, A\&A, 344, 231 
Kaufer, A., Stahl, O., Prinja, R. K., \& Witherick, D. 2006, A\&A, 447, 325

Kaufer, A., Stahl, O., Wolf, B., et al. 1997, A\&A, 320, 273

Kaufer, A., Stahl, O., Wolf, B., et al. 1996, A\&A, 314, 599

Kholopov, P. N., Samus', N. N., Frolov, M. S., et al. 1996, VizieR Online Data Catalog, 2139

Kholtygin, A. F., Castro, N., Fossati, L., et al. 2015, in Astronomical Society of the Pacific Conference Series, Vol. 494, Physics and Evolution of Magnetic and Related Stars, ed. Y. Y. Balega, I. I. Romanyuk, \& D. O. Kudryavtsev, 79

Kilian, J., Becker, S. R., Gehren, T., \& Nissen, P. E. 1991, A\&A, 244, 419

Klein, R. I. \& Castor, J. I. 1978, ApJ, 220, 902

Knoechel, G. \& Moffat, A. F. J. 1982, A\&A, 110, 263

Koen, C. \& Eyer, L. 2002, MNRAS, 331, 45

Kraus, M., Haucke, M., Cidale, L. S., et al. 2015, A\&A, 581, A75

Krtička, J. \& Kubát, J. 2001a, A\&A, 377, 175

Krtička, J. \& Kubát, J. 2001b, A\&A, 377, 175

Kudritzki, R.-P. 1980, A\&A, 85, 174

Kudritzki, R.-P., Lennon, D. J., \& Puls, J. 1995, in Science with the VLT, ed. J. R. Walsh \& I. J. Danziger, 246

Kudritzki, R. P., Pauldrach, A., Puls, J., \& Abbott, D. C. 1989, A\&A, 219, 205

Kudritzki, R. P., Puls, J., Lennon, D. J., et al. 1999, A\&A, 350, 970

Kurucz, R. L. 1979, ApJS, 40, 1

Lamers, H. J. 1972, in Colloquium on Supergiant Stars, ed. M. Hack, 83-84

Lamers, H. J. G. L. M. \& Cassinelli, J. P. 1999a, Introduction to Stellar Winds

Lamers, H. J. G. L. M. \& Cassinelli, J. P. 1999b, Introduction to Stellar Winds

Lamers, H. J. G. L. M., Gathier, R., \& Snow, Jr., T. P. 1982, ApJ, 258, 186

Lamers, H. J. G. L. M. \& Morton, D. C. 1976, ApJS, 32, 715

Lamers, H. J. G. L. M., Snow, T. P., \& Lindholm, D. M. 1995, ApJ, 455, 269 
Langer, N. 2012, ARA\&A, 50, 107

Lanz, T. \& Hubeny, I. 2007, ApJS, 169, 83

Le Pennec, M. \& Turck-Chièze, S. 2014, in IAU Symposium, Vol. 301, Precision Asteroseismology, ed. J. A. Guzik, W. J. Chaplin, G. Handler, \& A. Pigulski, 229-232

Lefever, K., Puls, J., \& Aerts, C. 2007, A\&A, 463, 1093

Lefever, K., Puls, J., Morel, T., et al. 2010, A\&A, 515, A74

Lefèvre, L., Marchenko, S. V., Moffat, A. F. J., \& Acker, A. 2009, A\&A, 507, 1141

Lennon, D. J., Dufton, P. L., \& Fitzsimmons, A. 1992, A\&AS, 94, 569

Lennon, D. J., Dufton, P. L., \& Fitzsimmons, A. 1993a, A\&AS, 97, 559

Lennon, D. J., Dufton, P. L., \& Fitzsimmons, A. 1993b, A\&AS, 97, 559

Lucy, L. B. 1976, ApJ, 206, 499

Lucy, L. B. \& Solomon, P. M. 1970, ApJ, 159, 879

MacGregor, K. B., Hartmann, L., \& Raymond, J. C. 1979, ApJ, 231, 514

Madura, T. I., Owocki, S. P., \& Feldmeier, A. 2007, ApJ, 660, 687

Maeder, A. \& Meynet, G. 2008, in Astronomical Society of the Pacific Conference Series, Vol. 388, Mass Loss from Stars and the Evolution of Stellar Clusters, ed. A. de Koter, L. J. Smith, \& L. B. F. M. Waters, 3

Maharramov, Y. M. 2013, Astronomy Reports, 57, 303

Mahy, L., Hutsemékers, D., Royer, P., \& Waelkens, C. 2016, A\&A, 594, A94

Marco, A. \& Negueruela, I. 2009, A\&A, 493, 79

Markova, N., Prinja, R. K., Markov, H., et al. 2008a, A\&A, 487, 211

Markova, N., Prinja, R. K., Markov, H., et al. 2008b, A\&A, 487, 211

Markova, N. \& Puls, J. 2008, A\&A, 478, 823

Markova, N., Puls, J., Repolust, T., \& Markov, H. 2004, A\&A, 413, 693

Markova, N., Puls, J., Simón-Díaz, S., et al. 2014, A\&A, 562, A37

Martins, F. \& Palacios, A. 2013, A\&A, 560, A16

Martins, F., Schaerer, D., \& Hillier, D. J. 2005, A\&A, 436, 1049 
Massa, D., Fullerton, A. W., Nichols, J. S., et al. 1995, ApJL, 452, L53

Massa, D., Fullerton, A. W., Sonneborn, G., \& Hutchings, J. B. 2003, ApJ, 586, 996

McErlean, N. D., Lennon, D. J., \& Dufton, P. L. 1999a, A\&A, 349, 553

McErlean, N. D., Lennon, D. J., \& Dufton, P. L. 1999b, A\&A, 349, 553

Megier, A., Strobel, A., Galazutdinov, G. A., \& Krełowski, J. 2009, A\&A, 507, 833

Meynet, G. \& Maeder, A. 2000, A\&A, 361, 101

Mihalas, D. 1978, Stellar atmospheres /2nd edition/

Milne, E. A. 1924, MNRAS, 84, 354

Milne, E. A. 1926, MNRAS, 86, 459

Mokiem, M. R., de Koter, A., Vink, J. S., et al. 2007, A\&A, 473, 603

Monteverde, M. I., Herrero, A., \& Lennon, D. J. 2000, ApJ, 545, 813

Moravveji, E., Guinan, E. F., Shultz, M., Williamson, M. H., \& Moya, A. 2012, ApJ, 747, 108

Morel, T., Hubrig, S., \& Briquet, M. 2008, A\&A, 481, 453

Morel, T., Marchenko, S. V., Pati, A. K., et al. 2004, MNRAS, 351, 552

Morgan, W. W. \& Roman, N. G. 1950, ApJ, 112, 362

Mullan, D. J. 1986, A\&A, 165, 157

Nerney, S. 1980, ApJ, 242, 723

North, P. \& Paltani, S. 1994, A\&A, 288, 155

Osterbrock, D. \& Flather, E. 1959, ApJ, 129, 26

Owocki, S. P., Castor, J. I., \& Rybicki, G. B. 1988, ApJ, 335, 914

Owocki, S. P. \& Rybicki, G. B. 1984, ApJ, 284, 337

Panei, J. 2004, PhD thesis, Universidad Nacional de La Plata

Pasinetti Fracassini, L. E., Pastori, L., Covino, S., \& Pozzi, A. 2001, A\&A, 367, 521

Pauldrach, A., Puls, J., \& Kudritzki, R. P. 1986, A\&A, 164, 86

Pauldrach, A. W. A., Hoffmann, T. L., \& Lennon, M. 2001, A\&A, 375, 161 
Paxton, B., Bildsten, L., Dotter, A., et al. 2011, ApJS, 192, 3

Paxton, B., Cantiello, M., Arras, P., et al. 2013, ApJS, 208, 4

Percy, J. R. \& Welch, D. L. 1983, PASP, 95, 491

Petrov, B., Vink, J. S., \& Gräfener, G. 2014, A\&A, 565, A62

Prinja, R. K., Barlow, M. J., \& Howarth, I. D. 1990a, ApJ, 361, 607

Prinja, R. K., Barlow, M. J., \& Howarth, I. D. 1990b, ApJ, 361, 607

Prinja, R. K. \& Howarth, I. D. 1986, ApJS, 61, 357

Prinja, R. K. \& Howarth, I. D. 1988, MNRAS, 233, 123

Prinja, R. K., Massa, D., \& Fullerton, A. W. 1995, ApJL, 452, L61

Prinja, R. K., Massa, D., \& Fullerton, A. W. 2002, A\&A, 388, 587

Prinja, R. K., Massa, D., Fullerton, A. W., Howarth, I. D., \& Pontefract, M. 1997, A\&A, 318, 157

Prinja, R. K. \& Massa, D. L. 2010a, A\&A, 521, L55

Prinja, R. K. \& Massa, D. L. 2010b, A\&A, 521, L55

Prinja, R. K. \& Massa, D. L. 2013, A\&A, 559, A15

Prinja, R. K., Rivinius, T., Stahl, O., et al. 2004, A\&A, 418, 727

Prugniel, P., Vauglin, I., \& Koleva, M. 2011, A\&A, 531, A165

Przybilla, N., Butler, K., Becker, S. R., \& Kudritzki, R. P. 2006, A\&A, 445, 1099

Puls, J. 1987, A\&A, 184, 227

Puls, J., Kudritzki, R.-P., Herrero, A., et al. 1996, A\&A, 305, 171

Puls, J., Markova, N., Scuderi, S., et al. 2006, A\&A, 454, 625

Puls, J., Urbaneja, M. A., Venero, R., et al. 2005, A\&A, 435, 669

Puls, J., Vink, J. S., \& Najarro, F. 2008, A\&A, 16, 209

Repolust, T., Puls, J., Hanson, M. M., Kudritzki, R.-P., \& Mokiem, M. R. 2005, A\&A, 440, 261

Rivinius, T., Stahl, O., Wolf, B., et al. 1997, A\&A, 318, 819

Rufener, F. \& Bartholdi, P. 1982, A\&AS, 48, 503 
Rusconi, L., Sedmak, G., Stalio, R., \& Arpigny, C. 1980, A\&AS, 42, 347

Ryans, R. S. I., Dufton, P. L., Rolleston, W. R. J., et al. 2002, MNRAS, 336, 577

Rzaev, A. K. 2012, Astrophysical Bulletin, 67, 282

Saio, H. 2009, Communications in Asteroseismology, 158, 245

Saio, H. 2011, MNRAS, 412, 1814

Saio, H., Baker, N. H., \& Gautschy, A. 1998, MNRAS, 294, 622

Saio, H., Georgy, C., \& Meynet, G. 2013a, MNRAS, 433, 1246

Saio, H., Georgy, C., \& Meynet, G. 2013b, MNRAS, 433, 1246

Saio, H., Kuschnig, R., Gautschy, A., et al. 2006, ApJ, 650, 1111

Santolaya-Rey, A. E., Puls, J., \& Herrero, A. 1997, A\&A, 323, 488

Scargle, J. D. 1982, ApJ, 263, 835

Schaller, G., Schaerer, D., Meynet, G., \& Maeder, A. 1992, A\&AS, 96, 269

Scuderi, S., Bonanno, G., di Benedetto, R., Spadaro, D., \& Panagia, N. 1992, ApJ, 392, 201

Scuderi, S., Panagia, N., Stanghellini, C., Trigilio, C., \& Umana, G. 1998, A\&A, 332, 251

Searle, S. C., Prinja, R. K., Massa, D., \& Ryans, R. 2008a, A\&A, 481, 777

Searle, S. C., Prinja, R. K., Massa, D., \& Ryans, R. 2008b, A\&A, 481, 777

Seward, F. D., Forman, W. R., Giacconi, R., et al. 1979, ApJL, 234, L55

Shibahashi, H. \& Osaki, Y. 1981, PASJ, 33, 427

Simón-Díaz, S., Caballero, J. A., Lorenzo, J., et al. 2015, ApJ, 799, 169

Simon-Diaz, S., Castro, N., Herrero, A., et al. 2013, in Massive Stars: From alpha to Omega, 28

Simón-Díaz, S. \& Herrero, A. 2007a, A\&A, 468, 1063

Simón-Díaz, S. \& Herrero, A. 2007b, A\&A, 468, 1063

Simón-Díaz, S. \& Herrero, A. 2014a, A\&A, 562, A135

Simón-Díaz, S. \& Herrero, A. 2014b, A\&A, 562, A135

Simón-Díaz, S., Herrero, A., Uytterhoeven, K., et al. 2010, ApJL, 720, L174 
Slettebak, A. \& Howard, R. F. 1955, ApJ, 121, 102

Smartt, S. J., Lennon, D. J., Kudritzki, R. P., et al. 2002, A\&A, 391, 979

Smith, N., Arnett, W. D., Bally, J., Ginsburg, A., \& Filippenko, A. V. 2013, MNRAS, 429, 1324

Smith, N., Bally, J., \& Walawender, J. 2007, AJ, 134, 846

Snow, Jr., T. P. \& Morton, D. C. 1976, ApJS, 32, 429

Sobolev, V. V. 1960, Moving envelopes of stars

Stahl, O., Kaufer, A., Wolf, B., et al. 1995, Journal of Astronomical Data, 1

Sterken, C. 1977, A\&A, 57, 361

Strömgren, B. 1966, ARA\&A, 4, 433

Sundqvist, J. O., Owocki, S. P., \& Puls, J. 2012, in Astronomical Society of the Pacific Conference Series, Vol. 465, Proceedings of a Scientific Meeting in Honor of Anthony F. J. Moffat, ed. L. Drissen, C. Robert, N. St-Louis, \& A. F. J. Moffat, 119

Takeda, Y. \& Takada-Hidai, M. 2000, PASJ, 52, 113

Underhill, A. B. 1960, PASP, 72, 363

Underhill, A. B. 1969, A\&A, 1, 494

Underhill, A. B. 1984, ApJ, 285, 668

van Breda, I. G., Glass, I. S., \& Whittet, D. C. B. 1974, MNRAS, 168, 551

van Genderen, A. M. 1989, A\&A, 208, 135

van Genderen, A. M., Bovenschen, H., Engelsman, E. C., et al. 1989, A\&AS, 79, 263

van Leeuwen, F. 2007a, A\&A, 474, 653

van Leeuwen, F. 2007b, A\&A, 474, 653

van Leeuwen, F., van Genderen, A. M., \& Zegelaar, I. 1998, A\&AS, 128, 117

Venero, R. O. J., Curé, M., Cidale, L. S., \& Araya, I. 2016, ApJ, 822, 28

Vink, J. S. 2000, PhD thesis, Universiteit Utrecht <EMAIL >jvink@phys.uu.nl</EMAIL>

Vink, J. S., de Koter, A., \& Lamers, H. J. G. L. M. 1999, A\&A, 350, 181

Vink, J. S., de Koter, A., \& Lamers, H. J. G. L. M. 2001, A\&A, 369, 574 
Wade, G. A., Neiner, C., Alecian, E., et al. 2016, MNRAS, 456, 2

Waelkens, C., Aerts, C., Kestens, E., Grenon, M., \& Eyer, L. 1998, A\&A, 330, 215

Walborn, N. R. 1971, ApJL, 164, L67

Walborn, N. R. 1976, ApJ, 205, 419

Wenger, M., Ochsenbein, F., Egret, D., et al. 2000, A\&AS, 143, 9

Wright, E. L., Eisenhardt, P. R. M., Mainzer, A. K., et al. 2010, AJ, 140, 1868

Zorec, J. \& Briot, D. 1991, A\&A, 245, 150

Zorec, J., Cidale, L., Arias, M. L., et al. 2009a, A\&A, 501, 297

Zorec, J., Cidale, L., Arias, M. L., et al. 2009b, A\&A, 501, 297

Zorec, J., Frémat, Y., \& Cidale, L. 2005, A\&A, 441, 235 